\title{
Multitargeted Compounds Derived from (2,5-Dioxopyrrolidin-1-yl)(phenyl)- Acetamides as Candidates for Effective Anticonvulsant and Antinociceptive Agents
}

Michał Abram ${ }^{\dagger}$, Anna Rapacz ${ }^{\#}$, Szczepan Mogilski ${ }^{\#}$, Gniewomir Latacz ${ }^{\ddagger}$, Annamaria Lubelska $^{\ddagger}$, Rafał M. Kamiński ${ }^{\dagger}$, and Krzysztof Kamiński ${ }^{\dagger}, *$

$\dagger$ Jagiellonian University Medical College, Faculty of Pharmacy, Department of Medicinal Chemistry, Medyczna 9, 30-688 Cracow, Poland; \# Jagiellonian University Medical College, Faculty of Pharmacy, Department of Pharmacodynamics, Medyczna 9, 30-688 Cracow, Poland; ${ }^{\ddagger}$ Jagiellonian University Medical College, Faculty of Pharmacy, Department of Technology and Biotechnology of Drugs, Medyczna 9, 30-688 Cracow, Poland

\section{Table of contents}

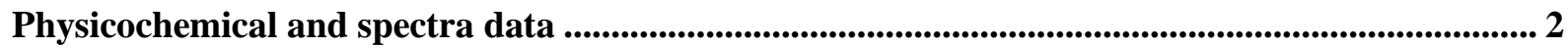

Boc-protected 4-phenylpiperazine derivatives A2-A12 and amines A14-A24 _............................ 2

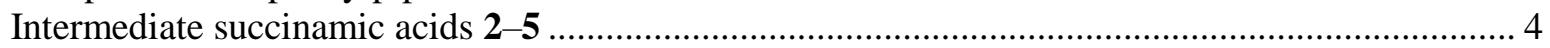

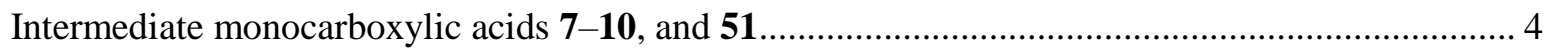

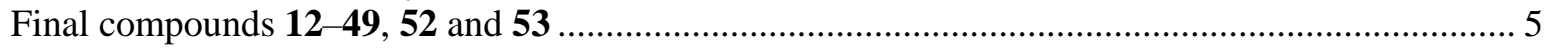

Table S1. Anticonvulsant activity-MES and $6 \mathrm{~Hz}(32 \mathrm{~mA})$ tests in mice i.p. (dose of $100 \mathrm{mg} / \mathrm{kg}$ ) -

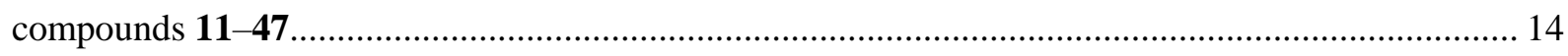

Table S2. Anticonvulsant activity-PTZ test in mice i.p. (dose of $100 \mathrm{mg} / \mathrm{kg}$ ) ............................... 15

Table S3. Anticonvulsant activity in mice i.p. (dose of $100 \mathrm{mg} / \mathrm{kg}$ ) - compounds 48, 49, 52, and $\mathbf{5 3} 15$

Table S4. The detailed data form $s c$ PTZ seizure test for compounds 11, 13, 19, and $\mathbf{2 2} \ldots \ldots \ldots \ldots \ldots \ldots . . . . . . .16$

Table S5. Binding/functional profile for anticonvulsant active $(\mathbf{2 7}, \mathbf{4 4})$ vs. inactive $(\mathbf{2 6})$ compounds 17

Figure S1 Anticonvulsant activity of compounds $\mathbf{1 1}$ and $\mathbf{1 9}$ in the $s c$ PTZ test............................... 18

Figure S2. The influence of compound 22 on spontaneous activity of mice....................................... 19

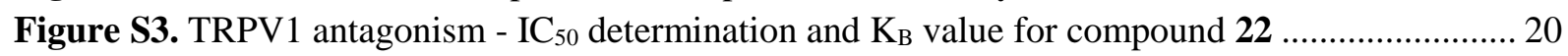

Figure S4. The in silico prediction of the most probably sites of metabolism of $\mathbf{2 2}$ by using MetaSite

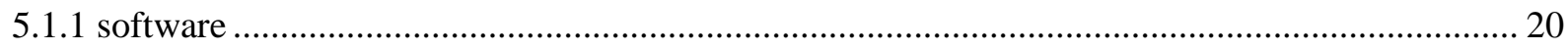

Figure S5. The UPLC spectrum after 120 min reaction of 22 with HLMs ..................................... 21

Figure S6. MS spectra of compound 22 and its metabolites M1-M3 obtained after incubation with

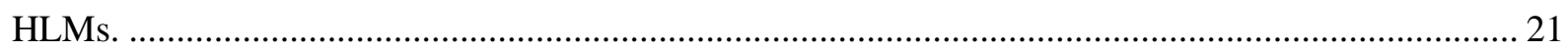

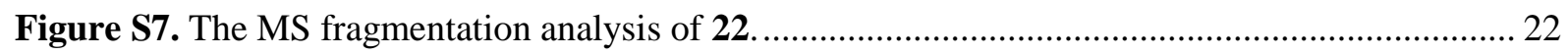

Figure S8. The most probably structures of metabolites M1-M3 based on the MetaSite metabolites'

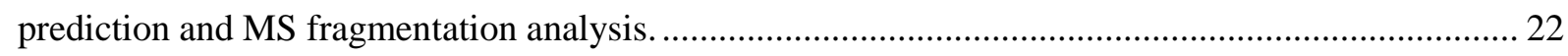

Figure S9. The influence of $\mathbf{2 2}$ and the reference inhibitor ketoconazole (KE) on CYP3A4 activity. 23 Figure S10. The influence of $\mathbf{2 2}$ and the reference inhibitor quinidine (QD) on CYP2D6 activity.... 23

In vitro ADME-Tox studies - materials and methods............................................................................... 24

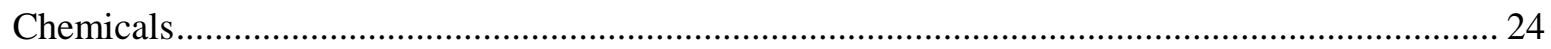

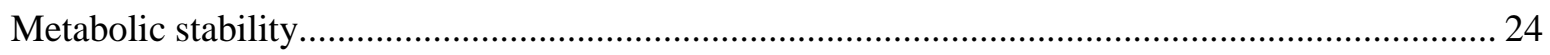

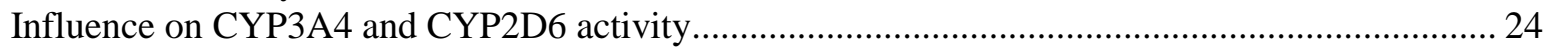

Radioligand binding/functional assays .................................................................................................... 25

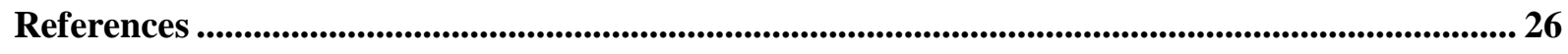

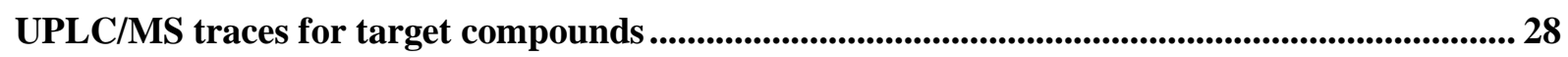

${ }^{1} \mathrm{H}$ NMR, ${ }^{13} \mathrm{C}$ NMR, ${ }^{19} \mathrm{~F}$ NMR spectra for selected starting amines, intermediates and final compounds ........................................................................................................................................................ 70 


\section{Physicochemical and spectra data}

\section{Boc-protected 4-phenylpiperazine derivatives A2-A12 and amines A14-A24}

Tert-butyl 4-(3-(tert-butyl)phenyl)piperazine-1-carboxylate (A2). Yellow oil, yield 79\% (2.42g); TLC: $R_{\mathrm{f}}=0.58\left(\mathrm{~S}_{3}\right)$; UPLC (purity $\left.72 \%\right): t_{\mathrm{R}}=9.19 \mathrm{~min}$. LC-MS (ESI): $\mathrm{m} / \mathrm{z}$ calcd for $\mathrm{C}_{19} \mathrm{H}_{30} \mathrm{~N}_{2} \mathrm{O}_{2}(\mathrm{M}+\mathrm{H})^{+}$319.24, found 319.2.

Tert-butyl 4-(3-(trifluoromethoxy)phenyl)piperazine-1-carboxylate (A3). Yellow oil, yield $78 \%(2.71 \mathrm{~g})$; TLC: $R_{\mathrm{f}}=0.52\left(\mathrm{~S}_{2}\right)$; UPLC (purity 98\%): $t_{\mathrm{R}}=8.73 \mathrm{~min}$. LC-MS (ESI): $\mathrm{m} / z$ calcd for $\mathrm{C}_{16} \mathrm{H}_{21} \mathrm{~F}_{3} \mathrm{~N}_{2} \mathrm{O}_{3}(\mathrm{M}+\mathrm{H})^{+} 347.16$, found 347.2.

Tert-butyl 4-([1,1'-biphenyl]-3-yl)piperazine-1-carboxylate (A4). Yellow oil, yield 74\% $(2.52 \mathrm{~g})$; TLC: $R_{\mathrm{f}}=0.57\left(\mathrm{~S}_{2}\right)$; UPLC (purity $\left.100 \%\right): t_{\mathrm{R}}=8.95 \mathrm{~min}$. LC-MS (ESI): $\mathrm{m} / z$ calcd for $\mathrm{C}_{21} \mathrm{H}_{26} \mathrm{~N}_{2} \mathrm{O}_{2}(\mathrm{M}+\mathrm{H})^{+}$339.23, found 339.2.

Tert-butyl 4-(3-(benzyloxy)phenyl)piperazine-1-carboxylate (A5). Yellow oil, yield 68\% $(2.49 \mathrm{~g})$; TLC: $R_{\mathrm{f}}=0.46\left(\mathrm{~S}_{3}\right)$; UPLC (purity $\left.87 \%\right): t_{\mathrm{R}}=8.84 \mathrm{~min}$. LC-MS (ESI): $\mathrm{m} / \mathrm{z}$ calcd for $\mathrm{C}_{22} \mathrm{H}_{28} \mathrm{~N}_{2} \mathrm{O}_{3}(\mathrm{M}+\mathrm{H})^{+} 369.22$, found 369.2.

Tert-butyl 4-(3-(methylthio)phenyl)piperazine-1-carboxylate (A6). Yellow oil, yield 72\% $(2.22 \mathrm{~g})$; TLC: $R_{\mathrm{f}}=0.91\left(\mathrm{~S}_{2}\right)$; UPLC (purity $\left.85 \%\right): t_{\mathrm{R}}=7.69 \mathrm{~min}$. LC-MS (ESI): $\mathrm{m} / \mathrm{z}$ calcd for $\mathrm{C}_{16} \mathrm{H}_{24} \mathrm{~N}_{2} \mathrm{O}_{2} \mathrm{~S}(\mathrm{M}+\mathrm{H})^{+}$308.16, found 308.2.

Tert-butyl 4-(3-((trifluoromethyl)thio)phenyl)piperazine-1-carboxylate (A7). Yellow oil, yield 75\% (2.72 g); TLC: $R_{\mathrm{f}}=0.74\left(\mathrm{~S}_{2}\right)$; UPLC (purity 91.4\%): $t_{\mathrm{R}}=9.14 \mathrm{~min}$. LC-MS (ESI): $m / z$ calcd for $\mathrm{C}_{16} \mathrm{H}_{21} \mathrm{~F}_{3} \mathrm{~N}_{2} \mathrm{O}_{2} \mathrm{~S}(\mathrm{M}+\mathrm{H})^{+} 363.13$, found 363.2.

Tert-butyl 4-(3-(difluoromethyl)phenyl)piperazine-1-carboxylate (A8). Yellow oil, yield $82 \%(2.57 \mathrm{~g})$; TLC: $R_{\mathrm{f}}=0.86\left(\mathrm{~S}_{2}\right)$; UPLC (purity $\left.87.8 \%\right): t_{\mathrm{R}}=7.83 \mathrm{~min}$. LC-MS (ESI): $\mathrm{m} / \mathrm{z}$ calcd for $\mathrm{C}_{16} \mathrm{H}_{22} \mathrm{~F}_{2} \mathrm{~N}_{2} \mathrm{O}_{2}(\mathrm{M}+\mathrm{H})^{+} 313.16$, found 313.4 .

Tert-butyl 4-(3,5-dichlorophenyl)piperazine-1-carboxylate (A9). Yellow oil, yield 71\% $(2.35 \mathrm{~g})$; TLC: $R_{\mathrm{f}}=0.80\left(\mathrm{~S}_{2}\right)$; UPLC (purity $\left.84.9 \%\right): t_{\mathrm{R}}=9.32 \mathrm{~min}$. LC-MS (ESI): $\mathrm{m} / z$ calcd for $\mathrm{C}_{15} \mathrm{H}_{20} \mathrm{Cl}_{2} \mathrm{~N}_{2} \mathrm{O}_{2}(\mathrm{M}+\mathrm{H})^{+} 331.09$, found 331.1.

Tert-butyl 4-(3,5-bis(trifluoromethyl)phenyl)piperazine-1-carboxylate (A10). Yellow oil, yield 62\% (2.48 g); TLC: $R_{\mathrm{f}}=0.89\left(\mathrm{~S}_{2}\right)$; UPLC (purity 100\%): $t_{\mathrm{R}}=9.34 \mathrm{~min}$. LC-MS (ESI): $m / z$ calcd for $\mathrm{C}_{17} \mathrm{H}_{20} \mathrm{~F}_{6} \mathrm{~N}_{2} \mathrm{O}_{2}(\mathrm{M}+\mathrm{H})^{+} 399.14$, found 399.5.

Tert-butyl 4-(3,5-bis((trifluoromethyl)thio)phenyl)piperazine-1-carboxylate (A11). Yellow oil, yield 67\% (3.10 g); TLC: $R_{\mathrm{f}}=0.78\left(\mathrm{~S}_{2}\right)$; UPLC (purity 94.2\%): $t_{\mathrm{R}}=9.42 \mathrm{~min}$. LCMS (ESI): $m / z$ calcd for $\mathrm{C}_{17} \mathrm{H}_{20} \mathrm{~F}_{6} \mathrm{~N}_{2} \mathrm{O}_{2} \mathrm{~S}_{2}(\mathrm{M}+\mathrm{H})^{+} 463.09$, found 463.2.

Tert-butyl 4-(3-chloro-5-(trifluoromethyl)phenyl)piperazine-1-carboxylate (A12). Yellow oil, yield 73\% (2.66 g); TLC: $R_{\mathrm{f}}=0.82\left(\mathrm{~S}_{2}\right)$; UPLC (purity $\left.85.7 \%\right): t_{\mathrm{R}}=9.27 \mathrm{~min}$. LC-MS (ESI): $m / z$ calcd for $\mathrm{C}_{16} \mathrm{H}_{20} \mathrm{ClF}_{3} \mathrm{~N}_{2} \mathrm{O}_{2}(\mathrm{M}+\mathrm{H})^{+} 365.12$, found 365.2.

1-(3-(Tert-butyl)phenyl)piperazine (A14). Yellow oil, yield 98\% (1.62 g); TLC: $R_{\mathrm{f}}=0.30$ $\left(\mathrm{S}_{3}\right)$; UPLC (purity 89\%): $t_{\mathrm{R}}=4.46 \mathrm{~min}$. LC-MS (ESI): $\mathrm{m} / z$ calcd for $\mathrm{C}_{14} \mathrm{H}_{22} \mathrm{~N}_{2}(\mathrm{M}+\mathrm{H})^{+} 219.18$, found 219.2. ${ }^{1} \mathrm{H}$ NMR $\left(300 \mathrm{MHz}, \mathrm{CDCl}_{3}\right) \delta 1.28-1.36\left(\mathrm{~m}, 10 \mathrm{H} ; 9 \mathrm{H}, \mathrm{C}\left(\mathrm{CH}_{3}\right)_{3}, 1 \mathrm{H}\right.$, piperazine), 3.07-3.28 (m, 8H, piperazine), 6.77 (dd, 1H, J=2.4, 0.8 Hz, ArH), 6.90-7.04 (m, 2H, ArH), $7.22(\mathrm{t}, 1 \mathrm{H}, J=7.9 \mathrm{~Hz}, \mathrm{ArH})$. 
1-(3-(Trifluoromethoxy)phenyl)piperazine (A15). Yellow oil, yield 97\% (1.87 g); TLC: $R_{\mathrm{f}}=$ $0.25\left(\mathrm{~S}_{3}\right)$; UPLC (purity 85\%): $t_{\mathrm{R}}=3.99 \mathrm{~min}$. LC-MS $(\mathrm{ESI}): \mathrm{m} / z$ calcd for $\mathrm{C}_{11} \mathrm{H}_{13} \mathrm{~F}_{3} \mathrm{~N}_{2} \mathrm{O}(\mathrm{M}+\mathrm{H})^{+}$ 247.10, found 247.1. ${ }^{1} \mathrm{H}$ NMR (300 MHz, $\left.\mathrm{CDCl}_{3}\right) \delta 1.23(\mathrm{~s}, 1 \mathrm{H}$, piperazine) $2.91-3.16(\mathrm{~m}, 8 \mathrm{H}$, piperazine) $6.59-6.85(\mathrm{~m}, 3 \mathrm{H}, \mathrm{ArH}), 7.19(\mathrm{t}, 1 \mathrm{H}, J=8.3 \mathrm{~Hz}, \mathrm{ArH})$.

1-([1,1'-Biphenyl]-3-yl)piperazine (A16). Yellow oil, yield 95\% (1.68 g); TLC: $R_{\mathrm{f}}=0.82\left(\mathrm{~S}_{3}\right)$; UPLC (purity 100\%): $t_{\mathrm{R}}=4.39 \mathrm{~min}$. LC-MS (ESI): $\mathrm{m} / z$ calcd for $\mathrm{C}_{16} \mathrm{H}_{18} \mathrm{~N}_{2}(\mathrm{M}+\mathrm{H})^{+} 239.15$, found 239.2. ${ }^{1} \mathrm{H}$ NMR $\left(300 \mathrm{MHz}, \mathrm{CDCl}_{3}\right) \delta 1.15-1.28(\mathrm{~m}, 1 \mathrm{H}$, piperazine), 3.14-3.76 $(\mathrm{m}, 8 \mathrm{H}$, piperazine), 6.65-7.73 (m, 9H, ArH).

1-(3-(Benzyloxy)phenyl)piperazine (A17). Yellow oil, yield 94\% (1.71 g); TLC: $R_{\mathrm{f}}=0.35$ $\left(\mathrm{S}_{3}\right)$; UPLC (purity $86 \%$ ): $t_{\mathrm{R}}=4.59 \mathrm{~min}$. LC-MS (ESI): $\mathrm{m} / z$ calcd for $\mathrm{C}_{17} \mathrm{H}_{20} \mathrm{~N}_{2} \mathrm{O}(\mathrm{M}+\mathrm{H})^{+}$ 269.16, found 269.2. ${ }^{1} \mathrm{H}$ NMR (300 MHz, $\left.\mathrm{CDCl}_{3}\right) \delta 1.28(\mathrm{~s}, 1 \mathrm{H}$, piperazine), 2.58-3.59 $(\mathrm{m}, 8 \mathrm{H}$, piperazine), $4.89-5.16\left(\mathrm{~m}, 2 \mathrm{H},-\mathrm{CH}_{2}-\mathrm{O}-\right), 6.22-6.73(\mathrm{~m}, 3 \mathrm{H}, \mathrm{ArH}), 6.85-7.76(\mathrm{~m}, 6 \mathrm{H}, \mathrm{ArH})$.

1-(3-(Methylthio)phenyl)piperazine (A18) Yellow oil, yield 98\% (1.47 g); TLC: $R_{\mathrm{f}}=0.51$ $\left(\mathrm{S}_{4}\right)$; UPLC (purity $98.6 \%$ ): $t_{\mathrm{R}}=3.20 \mathrm{~min}$. LC-MS (ESI): $\mathrm{m} / z$ calcd for $\mathrm{C}_{11} \mathrm{H}_{16} \mathrm{~N}_{2} \mathrm{~S}(\mathrm{M}+\mathrm{H})^{+}$ 209.10, found 209.1. ${ }^{1} \mathrm{H}$ NMR (500 MHz, $\left.\mathrm{CDCl}_{3}\right) \delta 1.65-1.71(\mathrm{~m}, 1 \mathrm{H}$, piperazine), $2.46(\mathrm{~s}, 3 \mathrm{H}$, $\mathrm{CH}_{3}$ ), 3.00-3.02 (m, 4H, piperazine), 3.12-3.14 (m, 4H, piperazine), 6.70 (m, 1H, ArH), 6.74 (d, $1 \mathrm{H}, J=7.6 \mathrm{~Hz}, \mathrm{ArH}), 6.81$ (t, 1H, $J=2.0 \mathrm{~Hz}, \operatorname{ArH}), 7.17$ (t, $1 \mathrm{H}, J=7.9 \mathrm{~Hz}, \operatorname{ArH})$

1-(3-((Trifluoromethyl)thio)phenyl)piperazine (A19) Yellow oil, yield 95\% (2.04 g); TLC: $R_{\mathrm{f}}=0.57\left(\mathrm{~S}_{4}\right)$; UPLC (purity 97\%): $t_{\mathrm{R}}=3.69 \mathrm{~min}$. LC-MS (ESI): $\mathrm{m} / z$ calcd for $\mathrm{C}_{11} \mathrm{H}_{13} \mathrm{~F}_{3} \mathrm{~N}_{2} \mathrm{~S}$ $(\mathrm{M}+\mathrm{H})^{+}$263.08, found 263.1. ${ }^{1} \mathrm{H} \mathrm{NMR}\left(500 \mathrm{MHz}, \mathrm{CDCl}_{3}\right) \delta 2.65-2.71(\mathrm{~m}, 1 \mathrm{H}$, piperazine), 3.10-3.16 (m, 3H, piperazine), 3.18-3.31 (m, 5H, piperazine), 6.99-7.01 (m, 1H, ArH), 7.09$7.16(\mathrm{~m}, 2 \mathrm{H}, \mathrm{ArH}), 7.25-7.30(\mathrm{~m}, 1 \mathrm{H}, \mathrm{ArH})$.

1-(3-(Difluoromethyl)phenyl)piperazine (A20) Yellow oil, yield 97\% (1.69 g); TLC: $R_{\mathrm{f}}=$ $0.71\left(\mathrm{~S}_{4}\right)$; UPLC (purity 96.3\%): $t_{\mathrm{R}}=3.01 \mathrm{~min}$. LC-MS (ESI): $\mathrm{m} / z$ calcd for $\mathrm{C}_{11} \mathrm{H}_{14} \mathrm{~F}_{2} \mathrm{~N}_{2}(\mathrm{M}+\mathrm{H})^{+}$ 213.11, found 212.9. ${ }^{1} \mathrm{H}$ NMR (500 MHz, $\left.\mathrm{CDCl}_{3}\right) \delta 2.63-2.66(\mathrm{~m}, 1 \mathrm{H}$, piperazine), 3.15-3.18 (m, 4H, piperazine), 3.27-3.30 (m, 4H, piperazine), 6.40-6.55 (m, 1H, $\left.\mathrm{CHF}_{2}\right), 6.60-6.73(\mathrm{~m}$, 1H, ArH), 6.96-7.03 (m, 1H, ArH), 7.05-7.11 (m, 1H ArH), 7.15-7.23 (m, 1H, ArH).

1-(3,5-Dichlorophenyl)piperazine (A21) Yellow oil, yield 94\% $(1.53 \mathrm{~g})$; TLC: $R_{\mathrm{f}}=0.62\left(\mathrm{~S}_{4}\right)$; UPLC (purity 83.4\%): $t_{\mathrm{R}}=3.99 \mathrm{~min}$. LC-MS (ESI): $\mathrm{m} / z$ calcd for $\mathrm{C}_{10} \mathrm{H}_{12} \mathrm{Cl}_{2} \mathrm{~N}_{2}(\mathrm{M}+\mathrm{H})^{+} 231.04$, found 231.0. ${ }^{1} \mathrm{H}$ NMR $\left(500 \mathrm{MHz}, \mathrm{CDCl}_{3}\right) \delta 2.02-2.18(\mathrm{~m}, 1 \mathrm{H}$, piperazine) $2.97-3.00(\mathrm{~m}, 4 \mathrm{H}$, piperazine), 3.11-3.14 (m, 4H, piperazine), 6.72 (s, 2H, ArH), 6.77 (s, 1H, ArH).

1-(3,5-Bis(trifluoromethyl)phenyl)piperazine (A22) Yellow oil, yield 98\% $(1.81 \mathrm{~g})$; TLC: $R_{\mathrm{f}}$ $=0.57\left(\mathrm{~S}_{4}\right) ;$ UPLC (purity $\left.100 \%\right): t_{\mathrm{R}}=4.80 \mathrm{~min}$. LC-MS $(\mathrm{ESI}): \mathrm{m} / \mathrm{z}$ calcd for $\mathrm{C}_{12} \mathrm{H}_{12} \mathrm{~F}_{6} \mathrm{~N}_{2}$ $(\mathrm{M}+\mathrm{H})^{+}$299.09, found 299.3. ${ }^{1} \mathrm{H} \mathrm{NMR}\left(500 \mathrm{MHz}, \mathrm{CDCl}_{3}\right) \delta 2.63$ (br. s, $1 \mathrm{H}$, piperazine), $3.03-$ $3.05(\mathrm{~m}, 4 \mathrm{H}$, piperazine), 3.23-3.25 (m, 4H, piperazine), $7.22(\mathrm{~s}, 2 \mathrm{H}, \mathrm{ArH}), 7.25-7.26(\mathrm{~m}, 1 \mathrm{H}$, $\mathrm{ArH})$.

1-(3,5-Bis((trifluoromethyl)thio)phenyl)piperazine (A23) Yellow oil, yield 95\% (2.30 g); TLC: $R_{\mathrm{f}}=0.62\left(\mathrm{~S}_{4}\right) ;$ UPLC (purity 98.2\%): $t_{\mathrm{R}}=4.62 \mathrm{~min}$. LC-MS (ESI): $\mathrm{m} / z$ calcd for $\mathrm{C}_{12} \mathrm{H}_{12} \mathrm{~F}_{6} \mathrm{~N}_{2} \mathrm{~S}_{2}(\mathrm{M}+\mathrm{H})^{+}$363.03, found 363.2. $\delta$ 2.63-2.70 (m, $1 \mathrm{H}$, piperazine), 3.08-3.12 (m, $4 \mathrm{H}$, piperazine), 3.21-3.34 (m, 4H, piperazine), 6.95-7.03 (m, 1H, ArH), 7.12-7.22 (m, 1H, ArH), 7.26-7.29 (m, 1H, ArH). 
1-(3-Chloro-5-(trifluoromethyl)phenyl)piperazine (A24) Yellow oil, yield 98\% (1.89 g);

TLC: $R_{\mathrm{f}}=0.57\left(\mathrm{~S}_{4}\right)$; UPLC (purity 97.1\%): $t_{\mathrm{R}}=4.53 \mathrm{~min}$. LC-MS (ESI): $\mathrm{m} / \mathrm{z}$ calcd for $\mathrm{C}_{11} \mathrm{H}_{12} \mathrm{ClF}_{3} \mathrm{~N}_{2}(\mathrm{M}+\mathrm{H})^{+}$265.06, found 265.2. ${ }^{1} \mathrm{H}$ NMR $\left(500 \mathrm{MHz}, \mathrm{CDCl}_{3}\right) \delta 2.64-2.73(\mathrm{~m}, 2 \mathrm{H}$, piperazine $), 3.01-3.06(\mathrm{~m}, 3 \mathrm{H}$, piperazine $), 3.20-3.26(\mathrm{~m}, 4 \mathrm{H}$, piperazine $), 6.97(\mathrm{~d}, 2 \mathrm{H}$, $J=9.68 \mathrm{~Hz}, \mathrm{ArH}), 7.00-7.03(\mathrm{~m}, 1 \mathrm{H}, \mathrm{ArH})$.

\section{Intermediate succinamic acids 2-5}

4-((1-Carboxy-2-phenylethyl)amino)-4-oxobutanoic acid (2). White solid. Yield: $88 \%$ (7.00 g); mp. 143.8-144.9 ${ }^{\circ} \mathrm{C}$; TLC: $\mathrm{R}_{\mathrm{f}}=0.52\left(\mathrm{~S}_{4}\right)$; UPLC (purity 100\%): $t_{\mathrm{R}}=3.25 \mathrm{~min}$. LC-MS (ESI): $m / z$ calcd for $\mathrm{C}_{13} \mathrm{H}_{15} \mathrm{NO}_{5}(\mathrm{M}+\mathrm{H})^{+} 266.10$, found 266.1 .

4-((Carboxy(3-fluorophenyl)methyl)amino)-4-oxobutanoic acid (3). White solid. Yield: $80 \%$ (6.46 g); mp. 136.2-137.5 ${ }^{\circ}$; TLC: $\mathrm{R}_{\mathrm{f}}=0.45\left(\mathrm{~S}_{4}\right)$; UPLC (purity 100\%): $t_{\mathrm{R}}=3.21 \mathrm{~min}$. LC-MS (ESI): $m / z$ calcd for $\mathrm{C}_{12} \mathrm{H}_{12} \mathrm{FNO}_{5}(\mathrm{M}+\mathrm{H})^{+} 270.08$, found 270.1 .

4-((Carboxy(4-fluorophenyl)methyl)amino)-4-oxobutanoic acid (4). White solid. Yield: $85 \%$ (6.86 g); mp. 125.7-126.9 ${ }^{\circ}$; TLC: $\mathrm{R}_{\mathrm{f}}=0.42\left(\mathrm{~S}_{4}\right)$; UPLC (purity 100\%): $t_{\mathrm{R}}=3.19 \mathrm{~min}$. LC-MS (ESI): $m / z$ calcd for $\mathrm{C}_{12} \mathrm{H}_{12} \mathrm{FNO}_{5}(\mathrm{M}+\mathrm{H})^{+} 270.08$, found 270.1 .

4-((Carboxy(3,4-difluorophenyl)methyl)amino)-4-oxobutanoic acid (5). White solid. Yield: 83\% (7.15 g); mp. 145.5-146.4 ${ }^{\circ} \mathrm{C}$; TLC: $\mathrm{R}_{\mathrm{f}}=0.28\left(\mathrm{~S}_{4}\right)$; UPLC (purity 100\%): $t_{\mathrm{R}}=3.33 \mathrm{~min}$. LC-MS (ESI): $m / z$ calcd for $\mathrm{C}_{12} \mathrm{H}_{11} \mathrm{~F}_{2} \mathrm{NO}_{5}(\mathrm{M}+\mathrm{H})^{+}$288.07, found 288.1.

\section{Intermediate monocarboxylic acids 7-10, and 51}

2-(2,5-Dioxopyrrolidin-1-yl)-3-phenylpropanoic acid (7). White solid. Yield: 94\% (4.64 g); mp. $123.2-124.7^{\circ} \mathrm{C}$; TLC: $\mathrm{R}_{\mathrm{f}}=0.71\left(\mathrm{~S}_{4}\right)$; UPLC (purity $\left.100 \%\right): t_{\mathrm{R}}=3.62 \mathrm{~min}$. LC-MS (ESI): $m / z$ calcd for $\mathrm{C}_{13} \mathrm{H}_{13} \mathrm{NO}_{4}(\mathrm{M}+\mathrm{H})^{+} 248.09$, found $248.1 .{ }^{1} \mathrm{H}$ NMR $\left(300 \mathrm{MHz}, \mathrm{DMSO}-\mathrm{D}_{6}\right) \delta 2.30$ 2.77 (m, 4H, imide), 3.21-3.59 (m, 2H Ar- $\underline{\mathrm{H}}_{2}-$ ), 4.84-5.14 (m, 1H, -Cㅌ- $\left.-\mathrm{COOH}\right), 7.00-7.40$ (m, 4H, ArH), 13.23 (br. s, 1H, $\mathrm{COO} \underline{\mathrm{H}}$ ).

2-(2,5-Dioxopyrrolidin-1-yl)-2-(3-fluorophenyl)acetic acid (8). White solid. Yield: 92\% (4.62 g); mp. $151.8-152.4^{\circ} \mathrm{C}$; TLC: $\mathrm{R}_{\mathrm{f}}=0.21\left(\mathrm{~S}_{4}\right)$; UPLC (purity 98.3\%): $t_{\mathrm{R}}=3.70 \mathrm{~min}$. LC-MS (ESI): $m / z$ calcd for $\mathrm{C}_{12} \mathrm{H}_{10} \mathrm{FNO}_{4}(\mathrm{M}+\mathrm{H})^{+} 252.06$, found $252.1 .{ }^{1} \mathrm{H}$ NMR (300 MHz, DMSOD $) \delta 2.75(\mathrm{~s}, 4 \mathrm{H}$, imide), $5.79(\mathrm{~s}, 1 \mathrm{H},-\mathrm{C} \underline{\mathrm{H}}-\mathrm{COOH}), 6.91-7.15(\mathrm{~m}, 1 \mathrm{H}, \mathrm{ArH}), 7.18-7.49$ (m, $3 \mathrm{H}, \mathrm{ArH}$ ), 13.21 (br. s, 1H, $\mathrm{COO} \underline{\mathrm{H}}$ ).

2-(2,5-Dioxopyrrolidin-1-yl)-2-(4-fluorophenyl)acetic acid (9). White solid. Yield: 88\% $(4.42 \mathrm{~g}) ; \mathrm{mp} .200 .2-201.9^{\circ} \mathrm{C}$; TLC: $\mathrm{R}_{\mathrm{f}}=0.25\left(\mathrm{~S}_{4}\right)$; UPLC (purity 98.1\%): $t_{\mathrm{R}}=3.69 \mathrm{~min}$. LC-MS (ESI): $m / z$ calcd for $\mathrm{C}_{12} \mathrm{H}_{10} \mathrm{FNO}_{4}(\mathrm{M}-\mathrm{H})^{+} 252.06$, found 252.1. ${ }^{1} \mathrm{H}$ NMR (300 MHz, DMSOD $\left.{ }_{6}\right) 2.74(\mathrm{~s}, 4 \mathrm{H}$, imide), 5.80 (s, 1H, - $\underline{\mathrm{H}}-\mathrm{COOH}), 7.04$ (t, 2H, J=8.8 Hz, ArH), 7.40-7.62 (m, $2 \mathrm{H}, \mathrm{ArH}), 13.22$ (br. s, $1 \mathrm{H}, \mathrm{COOH}$ ).

2-(3,4-Difluorophenyl)-2-(2,5-dioxopyrrolidin-1-yl)acetic acid (10). White solid. Yield: 91\% $(4.90 \mathrm{~g})$; mp. $159.5-160.3^{\circ} \mathrm{C}$; TLC: $\mathrm{R}_{\mathrm{f}}=0.35\left(\mathrm{~S}_{4}\right)$; UPLC (purity $\left.100 \%\right): t_{\mathrm{R}}=4.07 \mathrm{~min}$. LC-MS (ESI): $m / z$ calcd for $\mathrm{C}_{12} \mathrm{H}_{9} \mathrm{~F}_{2} \mathrm{NO}_{2}(\mathrm{M}-\mathrm{H})^{+}$268.05, found 268. ${ }^{1} \mathrm{H}$ NMR (300 MHz, DMSO-D 6 ) $\delta 2.75$ (s, 4H, imide), 5.77 (s, $1 \mathrm{H}-\mathrm{C} \underline{\mathrm{H}}-\mathrm{COOH}), 7.12-7.29$ (m, 2H, ArH), 7.44 (ddd, $1 \mathrm{H}, J=11.7$, 7.8, $2.0 \mathrm{~Hz}, \mathrm{ArH}), 13.23$ (br. s, $1 \mathrm{H}, \overline{\mathrm{COOH}}$ ). 
4-(2,5-Dioxopyrrolidin-1-yl)benzoic acid (51). White solid. Yield: 76\% (3.33 g); mp. 231$232.5^{\circ} \mathrm{C}$; TLC: $\mathrm{R}_{\mathrm{f}}=0.60\left(\mathrm{~S}_{4}\right)$; UPLC (purity 95.2\%): $t_{\mathrm{R}}=2.54 \mathrm{~min} . \mathrm{LC}-\mathrm{MS}(\mathrm{ESI}): \mathrm{m} / z$ calcd for $\mathrm{C}_{11} \mathrm{H}_{9} \mathrm{NO}_{4}(\mathrm{M}+\mathrm{H})^{+}$220.06, found 220.1. ${ }^{1} \mathrm{H}$ NMR (300 MHz, DMSO-D $) \delta$ 2.75-2.93 (m, 4H, imide), 7.37-7.49 (m, 2H, ArH), 8.07-8.17 (m, 2H, ArH), 13.07 (br. s, 1H, COOH $)$.

\section{Final compounds 12-49, 52 and 53}

1-(2-(4-(2-Chlorophenyl)piperazin-1-yl)-2-oxo-1-phenylethyl)pyrrolidine-2,5-dione (12). White solid. Yield: $80 \%(1.97 \mathrm{~g})$; mp. $189.5-191.2^{\circ} \mathrm{C}$; TLC: $\mathrm{R}_{\mathrm{f}}=0.38\left(\mathrm{~S}_{2}\right)$; UPLC (purity $100 \%): t_{\mathrm{R}}=6.65 \mathrm{~min}$. LC-MS (ESI): $\mathrm{m} / z$ calcd for $\mathrm{C}_{22} \mathrm{H}_{22} \mathrm{ClN}_{3} \mathrm{O}_{3}(\mathrm{M}+\mathrm{H})^{+} 412.13$, found 412.3 . ${ }^{1} \mathrm{H}$ NMR $\left(300 \mathrm{MHz}, \mathrm{CDCl}_{3}\right) \delta 2.54-2.78(\mathrm{~m}, 5 \mathrm{H}$; 4H, imide, $1 \mathrm{H}$, piperazine), 2.79-2.90 (m, $1 \mathrm{H}$, piperazine), $2.92-3.13(\mathrm{~m}, 2 \mathrm{H}$, piperazine), $3.18-3.31(\mathrm{~m}, 1 \mathrm{H}$, piperazine $), 3.32-3.44(\mathrm{~m}$, $1 \mathrm{H}$, piperazine), 3.73-3.86 (m, 1H, piperazine), 3.88-3.99 (m, 1H, piperazine), $6.12(\mathrm{~s}, 1 \mathrm{H}$, CHCO), 6.86-7.03 (m, 2H, ArH), 7.19 (td, 1H, J=7.7, 1.5 Hz, ArH), 7.27-7.51 (m, 6H, ArH); ${ }^{13} \mathrm{C}$ NMR $\left(75 \mathrm{MHz}, \mathrm{CDCl}_{3}\right) \delta 28.0,42.8,46.2,50.8,50.9,56.8,120.5,124.3,127.7,128.6$, 128.8, 129.8, 130.7, 133.0, 148.4, 165.0, 176.3. Anal. calcd for $\mathrm{C}_{22} \mathrm{H}_{22} \mathrm{ClN}_{3} \mathrm{O}_{3}$ (411.88): C: 64.15, H: 5.38, N: 10.20; Found C: 64.13, H: 5.39, N: 10.19.

1-(2-(4-(3-Chlorophenyl)piperazin-1-yl)-2-oxo-1-phenylethyl)pyrrolidine-2,5-dione (13). White solid. Yield: $81 \%(2.00 \mathrm{~g})$; mp. $128.1-129^{\circ} \mathrm{C}$; TLC: $\mathrm{R}_{\mathrm{f}}=0.51\left(\mathrm{~S}_{1}\right)$; UPLC (purity $100 \%$ ): $t_{\mathrm{R}}=6.72 \mathrm{~min}$. LC-MS (ESI): $\mathrm{m} / z$ calcd for $\mathrm{C}_{22} \mathrm{H}_{22} \mathrm{ClN}_{3} \mathrm{O}_{3}(\mathrm{M}+\mathrm{H})^{+} 412.13$, found 412.2 . ${ }^{1} \mathrm{H}$ NMR (300 MHz, $\mathrm{CDCl}_{3}$ ) $\delta 2.58-2.73$ (m, 4H, imide), 3.00 (br. s, 1H, piperazine), 3.27-3.53 (m, 3H, piperazine), 3.54-3.86 (m, 2H, piperazine), 4.17 (br. s, $2 \mathrm{H}$, piperazine), $6.02(\mathrm{~s}, 1 \mathrm{H}$, C트), 7.27-7.40 (m, 7H, ArH), 7.51-7.63 (m, 2H, ArH); $\left.{ }^{13} \mathrm{C} \mathrm{NMR} \mathrm{(75} \mathrm{MHz,} \mathrm{CDCl}_{3}\right) \delta 28.0$, 40.0, 43.3, 53.3, 53.7, 56.5, 118.9, 120.8, 128.9, 129.1, 129.3, 129.6, 131.4, 132.1, 135.9, 143.8, 165.5, 176.7. Anal. calcd for $\mathrm{C}_{22} \mathrm{H}_{22} \mathrm{ClN}_{3} \mathrm{O}_{3}$ (411.89): C: 64.15, H: 5.38, N: 10.20; Found C: 64.16, H: 5.40, N: 10.17.

1-(2-(4-(4-Chlorophenyl)piperazin-1-yl)-2-oxo-1-phenylethyl)pyrrolidine-2,5-dione (14). White solid. Yield: $83 \%$ (2.05 g); mp. $162.8-163.5^{\circ} \mathrm{C}$; TLC: $\mathrm{R}_{\mathrm{f}}=0.44\left(\mathrm{~S}_{2}\right)$; UPLC (purity $100 \%$ ): $t_{\mathrm{R}}=6.67 \mathrm{~min}$. LC-MS (ESI): $\mathrm{m} / z$ calcd for $\mathrm{C}_{22} \mathrm{H}_{22} \mathrm{ClN}_{3} \mathrm{O}_{3}(\mathrm{M}+\mathrm{H})^{+} 412.13$, found $412.4 .{ }^{1} \mathrm{H}$ NMR $\left(300 \mathrm{MHz}, \mathrm{CDCl}_{3}\right) \delta 2.59-2.78(\mathrm{~m}, 5 \mathrm{H} ; 4 \mathrm{H}$, imide, $1 \mathrm{H}$, piperazine), 2.94-3.08 (m, 2H, piperazine), $3.14-3.37(\mathrm{~m}, 3 \mathrm{H}$, piperazine $), 3.61-3.76(\mathrm{~m}, 1 \mathrm{H}$, piperazine $), 3.90-4.03(\mathrm{~m}, 1 \mathrm{H}$, piperazine), 6.11 (s, 1H, CHCO), 6.69-6.78 (m, 2H, ArH), 7.13-7.21 (m, 2H, ArH), 7.29-7.47 $(\mathrm{m}, 5 \mathrm{H}, \mathrm{ArH}) ;{ }^{13} \mathrm{C}$ NMR $\left(75 \mathrm{MHz}, \mathrm{CDCl}_{3}\right) \delta 28.0,42.3,45.6,48.9,49.1,56.7,117.8,125.5$, 128.7, 128.9, 129.1, 129.8, 132.9, 149.3, 165.0, 176.3. Anal. calcd for $\mathrm{C}_{22} \mathrm{H}_{22} \mathrm{ClN}_{3} \mathrm{O}_{3}(411.89)$ : C: $64.15, \mathrm{H}: 5.38, \mathrm{~N}: 10.20$; Found C: 64.20, H: 5.36, N: 10.30.

1-(2-(4-(2-Fluorophenyl)piperazin-1-yl)-2-oxo-1-phenylethyl)pyrrolidine-2,5-dione (15). White solid. Yield: $69 \%$ (1.64g); mp. 211.2-212.5 ${ }^{\circ}$; TLC: $\mathrm{R}_{\mathrm{f}}=0.46\left(\mathrm{~S}_{2}\right)$; UPLC (purity 100\%): $t_{\mathrm{R}}=6.10 \mathrm{~min} \mathrm{LC}-\mathrm{MS}(\mathrm{ESI}): \mathrm{m} / z$ calcd for $\mathrm{C}_{22} \mathrm{H}_{22} \mathrm{FN}_{3} \mathrm{O}_{3}(\mathrm{M}+\mathrm{H})^{+} 396.16$, found 396.4. ${ }^{1} \mathrm{H} \mathrm{NMR}$ $\left(300 \mathrm{MHz}, \mathrm{CDCl}_{3}\right) \delta 2.53-2.78(\mathrm{~m}, 5 \mathrm{H} ; 4 \mathrm{H}$ imide, $1 \mathrm{H}$, piperazine $), 2.85-2.92(\mathrm{~m}, 1 \mathrm{H}$, piperazine), 2.97-3.17 (m, 2H, piperazine), 3.21-3.44 (m, 2H, piperazine), 3.68-3.84 (m, 1H, piperazine), 3.87-4.01 (m, 1H, piperazine), 6.12 (s, 1H, CㅂCO), 6.79-6.88 (m, 1H, ArH), 6.89-7.07 (m, 3H, ArH), 7.28-7.48 (m, 5H, ArH); ${ }^{13} \mathrm{C}$ NMR $\left(75 \mathrm{MHz}, \mathrm{CDCl}_{3}\right) \delta$ 28.0, 42.6, 46.0, 50.1, 56.7, 116.2 (d, $J=20.7 \mathrm{~Hz}), 119.1,123.2$ (d, $J=8.1 \mathrm{~Hz}), 124.5,128.6,128.8,129.8$, 133.0, 139.3 (d, $J=8.1 \mathrm{~Hz}), 154.0,157.3,165.0,176.3 ;{ }^{19} \mathrm{~F} \mathrm{NMR}\left(282 \mathrm{MHz}, \mathrm{CDCl}_{3}\right) \delta-123.00$ (br. s, 1F). Anal. calcd for $\mathrm{C}_{22} \mathrm{H}_{22} \mathrm{FN}_{3} \mathrm{O}_{3}$ (395.43): C: 66.82, $\mathrm{H}: 5.61, \mathrm{~N}: 10.63$; Found C: 66.78, H: 5.59, N: 10.65 . 
1-(2-(4-(3-Fluorophenyl)piperazin-1-yl)-2-oxo-1-phenylethyl)pyrrolidine-2,5-dione (16). White solid. Yield: $77 \%(1.92 \mathrm{~g})$; mp. $182.1-183.5^{\circ} \mathrm{C}$; TLC: $\mathrm{R}_{\mathrm{f}}=0.42\left(\mathrm{~S}_{1}\right)$; UPLC (purity 98.45\%): $t_{\mathrm{R}}=6.18 \mathrm{~min}$. LC-MS (ESI): $\mathrm{m} / \mathrm{z}$ calcd for $\mathrm{C}_{22} \mathrm{H}_{22} \mathrm{FN}_{3} \mathrm{O}_{3}(\mathrm{M}+\mathrm{H})^{+} 396.16$, found 396.5 . ${ }^{1} \mathrm{H}$ NMR $\left(300 \mathrm{MHz}, \mathrm{CDCl}_{3}\right) \delta 2.51-2.66(\mathrm{~m}, 4 \mathrm{H}$, imide), 2.72-2.87 (m, 1H, piperazine), 3.033.57 (m, 5H, piperazine), 3.94(br. s, $2 \mathrm{H}$, piperazine), 5.99 (s, $1 \mathrm{H}, \mathrm{CHCO}), 6.72(\mathrm{t}, 1 \mathrm{H}, J=8.1$, $1.5 \mathrm{~Hz}, \mathrm{ArH}), 6.82-7.00(\mathrm{~m}, 1 \mathrm{H}, \mathrm{ArH}), 7.15-7.33(\mathrm{~m}, 7 \mathrm{H}, \mathrm{ArH}) ;{ }^{13} \mathrm{C} \mathrm{NMR}\left(75 \mathrm{MHz}, \mathrm{CDCl}_{3}\right)$ $\delta 27.9,40.9,44.2,48.2,48.4,48.7,49.0,49.3,51.0,51.3,56.5,106.0$ (d, J=25.4 Hz), 110.0, 111.7, 114.1, 128.7, 129.0, 129.5, 130.9, 131.0, 132.2, 161.6, 165.5, 176.8. ${ }^{19}$ F NMR $(282 \mathrm{MHz}$, $\left.\mathrm{CDCl}_{3}\right) \delta-109.91$ (br. s, 1F). Anal. calcd for $\mathrm{C}_{22} \mathrm{H}_{22} \mathrm{FN}_{3} \mathrm{O}_{3}$ (395.43): C: 66.82, H: 5.61, N: 10.63; Found C: 66.88, H: 5.65, N: 10.61.

1-(2-(4-(4-Fluorophenyl)piperazin-1-yl)-2-oxo-1-phenylethyl)pyrrolidine-2,5-dione (17). White solid. Yield: $76 \%(1.80 \mathrm{~g})$; mp. $141.2-142.5^{\circ} \mathrm{C}$; TLC: $\mathrm{R}_{\mathrm{f}}=0.43\left(\mathrm{~S}_{2}\right)$; UPLC (purity $100 \%): t_{\mathrm{R}}=5.93 \mathrm{~min}$. LC-MS (ESI): $\mathrm{m} / z$ calcd for $\mathrm{C}_{22} \mathrm{H}_{22} \mathrm{FN}_{3} \mathrm{O}_{3}(\mathrm{M}+\mathrm{H})^{+} 396.16$, found 396.4 ${ }^{1} \mathrm{H}$ NMR $\left(300 \mathrm{MHz}, \mathrm{CDCl}_{3}\right) \delta 2.54-2.78(\mathrm{~m}, 5 \mathrm{H} ; 4 \mathrm{H}$, imide, $1 \mathrm{H}$, piperazine), 2.84-3.07 (m, $2 \mathrm{H}$, piperazine), 3.08-3.41 (m, 3H, piperazine), 3.63-3.77 (m, 1H, piperazine), 3.90-4.03 (m, $1 \mathrm{H}$, piperazine), 6.11 (s, 1H, CㅂCO), 6.74-6.83 (m, 2H, ArH), 6.88-6.98 (m, 2H, ArH), 7.28$7.47(\mathrm{~m}, 5 \mathrm{H}, \mathrm{ArH}) ;{ }^{13} \mathrm{C} \mathrm{NMR}\left(75 \mathrm{MHz}, \mathrm{CDCl}_{3}\right) \delta 28.0,42.5,45.8,49.9,50.2,56.7,115.7(\mathrm{~d}$, $J=21.9 \mathrm{~Hz}), 118.5$ (d, $J=8.1 \mathrm{~Hz}), 128.6,128.9,129.8,132.9,147.4,156.0,159.2,165.0,176.3$; ${ }^{19} \mathrm{~F} \mathrm{NMR}\left(282 \mathrm{MHz}, \mathrm{CDCl}_{3}\right) \delta-123.21$ (br. s, 1F). Anal. calcd for $\mathrm{C}_{22} \mathrm{H}_{22} \mathrm{FN}_{3} \mathrm{O}_{3}$ (395.43): C: 66.82, H: 5.61, N: 10.63; Found C: 66.79, H: 5.62, N: 10.59 .

1-(2-Oxo-1-phenyl-2-(4-(o-tolyl)piperazin-1-yl)ethyl)pyrrolidine-2,5-dione (18). White solid. Yield: $75 \%(1.76 \mathrm{~g})$; mp. $187.2-188.1^{\circ} \mathrm{C}$; TLC: $\mathrm{R}_{\mathrm{f}}=0.41\left(\mathrm{~S}_{2}\right)$; UPLC (purity $\left.100 \%\right): t_{\mathrm{R}}=$ 6.72 min LC-MS (ESI): $\mathrm{m} / z$ calcd for $\mathrm{C}_{23} \mathrm{H}_{25} \mathrm{~N}_{3} \mathrm{O}_{3}(\mathrm{M}+\mathrm{H})^{+} 392.19$, found 392.3. ${ }^{1} \mathrm{H}$ NMR (300 $\left.\mathrm{MHz} \mathrm{CDCl}_{3}\right) \delta 2.25\left(\mathrm{~s}, 3 \mathrm{H},-\mathrm{CH}_{3}\right), 2.44-2.56(\mathrm{~m}, 1 \mathrm{H}$, piperazine), 2.59-2.78 (m, 5H; $4 \mathrm{H}$, imide, $1 \mathrm{H}$, piperazine), 2.79-2.98 ( $\mathrm{m}, 2 \mathrm{H}$, piperazine), 3.16-3.42 (m, 2H, piperazine), 3.70$3.98(\mathrm{~m}, 2 \mathrm{H}$, piperazine), 6.14 (s, 1H, CㅂCO), 6.90 (d, 1H, J=7.8 Hz, ArH), 6.94-7.02 (m, 1H, ArH), 7.10-7.19 (m, 2H, ArH), 7.30-7.50 (m, 5H, ArH); $\left.{ }^{13} \mathrm{C} \mathrm{NMR} \mathrm{(75} \mathrm{MHz,} \mathrm{CDCl}_{3}\right) \delta 17.7$, 28.1, 43.1, 46.4, 51.3, 51.5, 56.8, 119.1, 123.8, 126.7, 128.6, 128.8, 129.9, 131.1, 132.6, 133.0, 150.6, 165.0, 176.4. Anal. calcd for $\mathrm{C}_{23} \mathrm{H}_{25} \mathrm{~N}_{3} \mathrm{O}_{3}$ (391.47): C: 70.57, H: 6.44, N: 10.73; Found C: $70.55, \mathrm{H}: 6.45, \mathrm{~N}: 10.79$.

1-(2-Oxo-1-phenyl-2-(4-(m-tolyl)piperazin-1-yl)ethyl)pyrrolidine-2,5-dione (19). White solid. Yield: $86 \%$ (2.02 g); mp. $188.7-192.1^{\circ} \mathrm{C}$; TLC: $\mathrm{R}_{\mathrm{f}}=0.45\left(\mathrm{~S}_{2}\right)$; UPLC (purity 98.92\%): $t_{\mathrm{R}}$ $=6.40$ min. LC-MS (ESI): $\mathrm{m} / z$ calcd for $\mathrm{C}_{23} \mathrm{H}_{25} \mathrm{~N}_{3} \mathrm{O}_{3}(\mathrm{M}+\mathrm{H})^{+} 392.19$, found 392.1. ${ }^{1} \mathrm{H} \mathrm{NMR}$ $\left(300 \mathrm{MHz}, \mathrm{CDCl}_{3}\right) \delta 2.36$ (s, 3H, - $\left.\underline{\mathrm{H}}_{3}\right), 2.57-2.78$ (m, 5H; $4 \mathrm{H}$, imide, $1 \mathrm{H}$, piperazine), $2.91-$ $3.54(\mathrm{~m}, 3 \mathrm{H}$, piperazine), 3.63-4.55 (m, $4 \mathrm{H}$, piperazine), $6.06(\mathrm{~s}, 1 \mathrm{H}, \mathrm{CHCO}), 7.22(\mathrm{~d}, 1 \mathrm{H}$, $J=7.5 \mathrm{~Hz}, \mathrm{ArH}), 7.27-7.62(\mathrm{~m}, 8 \mathrm{H}, \mathrm{ArH}) ;{ }^{13} \mathrm{C} \mathrm{NMR}\left(75 \mathrm{MHz}, \mathrm{CDCl}_{3}\right) \delta 21.4,28.1,39.7,43.0$, 54.1, 54.6, 56.5, 117.9, 121.7, 128.9, 129.3, 129.7, 130.2, 130.8, 132.3, 141.0, 141.8, 165.4, 176.3. Anal. calcd for $\mathrm{C}_{23} \mathrm{H}_{25} \mathrm{~N}_{3} \mathrm{O}_{3}$ (391.47): C: 70.57, H: 6.44, N: 10.73; Found C: 70.60, H: 6.48, N: 10.71 .

1-(2-Oxo-1-phenyl-2-(4-(p-tolyl)piperazin-1-yl)ethyl)pyrrolidine-2,5-dione (20). White solid. Yield: $77 \%(1.81 \mathrm{~g})$; mp. $140.2-141.8^{\circ} \mathrm{C}$; TLC: $\mathrm{R}_{\mathrm{f}}=0,28\left(\mathrm{~S}_{2}\right)$; UPLC (purity 100\%): $t_{\mathrm{R}}=$ 6.22 min. LC-MS (ESI): $\mathrm{m} / z$ calcd for $\mathrm{C}_{23} \mathrm{H}_{25} \mathrm{~N}_{3} \mathrm{O}_{3}(\mathrm{M}+\mathrm{H}){ }^{+}$392.19, found 391.9. ${ }^{1} \mathrm{H}$ NMR (300 $\left.\mathrm{MHz} \mathrm{CDCl}_{3}\right) \delta 2.26\left(\mathrm{~s}, 3 \mathrm{H},-\mathrm{CH}_{3}\right), 2.57-2.81(\mathrm{~m}, 5 \mathrm{H}$; $4 \mathrm{H}$, imide, $1 \mathrm{H}$, piperazine), 2.88-3.10 (m, $2 \mathrm{H}$, piperazine), 3.12-3.42 (m, 3H, piperazine), 3.63-3.77 (m, $1 \mathrm{H}$, piperazine), $3.90-4.05$ (m, 1H, piperazine), $6.12(\mathrm{~s}, 1 \mathrm{H}, \mathrm{C} \underline{\mathrm{HCO}}), 6.72-6.79(\mathrm{~m}, 2 \mathrm{H}, \mathrm{ArH}), 7.05(\mathrm{~d}, 2 \mathrm{H}, J=8.2 \mathrm{~Hz}$, ArH), 7.28-7.48 (m, 5H, ArH); ${ }^{13} \mathrm{C} \mathrm{NMR}\left(75 \mathrm{MHz}, \mathrm{CDCl}_{3}\right) \delta 20.4,28.0,42.5,45.8,49.5,49.7$, 56.8, 116.9, 128.6, 128.8, 129.7, 129.9, 130.2, 133.0, 148.6, 164.9, 176.3. . Anal. calcd for $\mathrm{C}_{23} \mathrm{H}_{25} \mathrm{~N}_{3} \mathrm{O}_{3}$ (391.47): C: 70.57, H: 6.44, N: 10.73; Found C: 70.51, H: 6.40, N: 10.77. 
1-(2-Oxo-1-phenyl-2-(4-(2-(trifluoromethyl)phenyl)piperazin-1-yl)ethyl)pyrrolidine-2,5dione (21). White solid. Yield: 69\% (1.84 g); mp. 167.1-168.7 ${ }^{\circ} \mathrm{C}$; TLC: $\mathrm{R}_{\mathrm{f}}=0.25\left(\mathrm{~S}_{2}\right)$; UPLC (purity $100 \%$ ): $t_{\mathrm{R}}=7.08 \mathrm{~min}$. LC-MS (ESI): $\mathrm{m} / z$ calcd for $\mathrm{C}_{23} \mathrm{H}_{22} \mathrm{~F}_{3} \mathrm{~N}_{3} \mathrm{O}_{3}(\mathrm{M}+\mathrm{H})^{+} 446.16$, found 446.2. ${ }^{1} \mathrm{H}$ NMR (300 MHz, $\left.\mathrm{CDCl}_{3}\right) \delta 2.44-2.55(\mathrm{~m}, 1 \mathrm{H}$, piperazine), 2.59-2.79 (m, 5H; 4H, imide, $1 \mathrm{H}$, piperazine), 2.82-2.98 (m, $2 \mathrm{H}$, piperazine), 3.15-3.22 (m, $1 \mathrm{H}$, piperazine), 3.29-

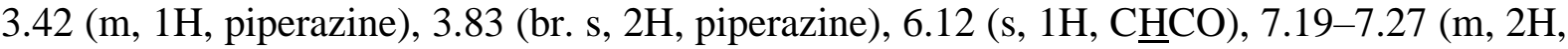
ArH), 7.29-7.54 (m, 6H, ArH), $7.60(\mathrm{~d}, 1 \mathrm{H}, J=8.1 \mathrm{~Hz}, \mathrm{ArH}) ;{ }^{13} \mathrm{C}$ NMR $\left(75 \mathrm{MHz}, \mathrm{CDCl}_{3}\right) \delta$ 28.0, 43.0, 46.4, 52.9, 56.8, 123.8 (q, $J=272.9 \mathrm{~Hz}), 124.0,125.5,127.2$ (q, J=5.7 Hz), 128.6, 128.8, 129.8, 132.8, 133.0, 151.4, 165.0, 176.3; ${ }^{19} \mathrm{~F} \mathrm{NMR}\left(282 \mathrm{MHz}, \mathrm{CDCl}_{3}\right) \delta-60.46(\mathrm{~s}, 3 \mathrm{~F})$. Anal. calcd for $\mathrm{C}_{23} \mathrm{H}_{22} \mathrm{~F}_{3} \mathrm{~N}_{3} \mathrm{O}_{3}$ (445.44): C: 62.02, $\mathrm{H}: 4.98$, N: 9.43; Found C: 62.08, $\mathrm{H}: 4.97$, $\mathrm{N}: 9.46$.

1-(2-Oxo-1-phenyl-2-(4-(3-(trifluoromethyl)phenyl)piperazin-1-yl)ethyl)pyrrolidine-2,5dione (22). White solid. Yield: $82 \%(2.19 \mathrm{~g})$; mp. $150.3-151.4^{\circ} \mathrm{C}$; TLC: $\mathrm{R}_{\mathrm{f}}=0.34\left(\mathrm{~S}_{1}\right)$; UPLC (purity $100 \%$ ): $t_{\mathrm{R}}=6.99 \mathrm{~min}$. LC-MS (ESI): $\mathrm{m} / z$ calcd for $\mathrm{C}_{23} \mathrm{H}_{22} \mathrm{~F}_{3} \mathrm{~N}_{3} \mathrm{O}_{3}(\mathrm{M}+\mathrm{H})^{+} 446.16$, found 446.2. ${ }^{1} \mathrm{H}$ NMR $\left(300 \mathrm{MHz}, \mathrm{CDCl}_{3}\right) \delta 2.60-2.86(\mathrm{~m}, 5 \mathrm{H}$; 4H, imide, $1 \mathrm{H}$, piperazine), 3.00-3.20 (m, $2 \mathrm{H}$, piperazine), 3.23-3.44 (m, 3H, piperazine), 3.62-3.76 $(\mathrm{m}, 1 \mathrm{H}$, piperazine $), 3.93-4.06$ (m, 1H, piperazine), 6.12 (s, 1H, C $\underline{H C O}), 6.94-7.04(\mathrm{~m}, 2 \mathrm{H}, \mathrm{ArH}), 7.09(\mathrm{~d}, 1 \mathrm{H}, J=7.7 \mathrm{~Hz}, \mathrm{ArH})$, 7.28-7.51 (m, 6H, ArH); $\left.{ }^{13} \mathrm{C} \mathrm{NMR} \mathrm{(75} \mathrm{MHz,} \mathrm{CDCl}_{3}\right) \delta$ 28.0, 42.2, 45.6, 48.4, 48.6, 56.8, 112.7 (q, $J=4.6 \mathrm{~Hz}), 116.7(\mathrm{q}, J=4.6 \mathrm{~Hz}), 119.2,123.4(\mathrm{q}, J=271.8 \mathrm{~Hz}), 128.7,128.9,129.7,129.8$, 131.5 (q, $J=31.8 \mathrm{~Hz}), 132.9,150.8,165.1,176.3 ;{ }^{19} \mathrm{~F} \mathrm{NMR}\left(282 \mathrm{MHz}, \mathrm{CDCl}_{3}\right) \delta-62.77$ (br. s, 3F). Anal. calcd for $\mathrm{C}_{23} \mathrm{H}_{22} \mathrm{~F}_{3} \mathrm{~N}_{3} \mathrm{O}_{3}$ (445.44): C: 62.02, $\mathrm{H}: 4.98, \mathrm{~N}: 9.43$; Found C: 62.05, H: 5.00, N: 9.40 .

1-(2-Oxo-1-phenyl-2-(4-(4-(trifluoromethyl)phenyl)piperazin-1-yl)ethyl)pyrrolidine-2,5-

dione (23). White solid. Yield: $62 \%(1.66 \mathrm{~g})$; mp. $173.2-174.3^{\circ} \mathrm{C}$; TLC: $\mathrm{R}_{\mathrm{f}}=0.49\left(\mathrm{~S}_{2}\right)$; UPLC (purity $100 \%$ ): $t_{\mathrm{R}}=6.99 \mathrm{~min}$. LC-MS (ESI): $\mathrm{m} / z$ calcd for $\mathrm{C}_{23} \mathrm{H}_{22} \mathrm{~F}_{3} \mathrm{~N}_{3} \mathrm{O}_{3}(\mathrm{M}+\mathrm{H})^{+} 446.16$, found 446.2. ${ }^{1} \mathrm{H}$ NMR $\left(300 \mathrm{MHz}, \mathrm{CDCl}_{3}\right) \delta 2.61-2.85(\mathrm{~m}, 5 \mathrm{H} ; 4 \mathrm{H}$, imide, $1 \mathrm{H}$, piperazine), 3.04-3.43 (m, 5H, piperazine), 3.63-3.77 $(\mathrm{m}, 1 \mathrm{H}$, piperazine $), 3.91-4.05(\mathrm{~m}, 1 \mathrm{H}$, piperazine $), 6.12(\mathrm{~s}, 1 \mathrm{H}$, CHCO), 6.83 (d, 2H, J=8.6 Hz, ArH), 7.30-7.40 (m, 3H, ArH), 7.40-7.50 (m, 4H, ArH); ${ }^{13} \mathrm{C}$ NMR $\left(75 \mathrm{MHz}, \mathrm{CDCl}_{3}\right) \delta 28.0,42.1,45.4,47.6,47.9,56.8,115.0,124.5$ (q, J=270.6 Hz), 126.5 $(\mathrm{q}, J=4.6 \mathrm{~Hz}), 128.7,128.8,128.9,129.8,132.8,152.7,165.1,176.3 ;{ }^{19} \mathrm{~F}$ NMR $(282 \mathrm{MHz}$, $\left.\mathrm{CDCl}_{3}\right) \delta-61.5(\mathrm{~s}, 3 \mathrm{~F})$. Anal. calcd for $\mathrm{C}_{23} \mathrm{H}_{22} \mathrm{~F}_{3} \mathrm{~N}_{3} \mathrm{O}_{3}$ (445.44): C: 62.02, H: 4.98, N: 9.43; Found C: 62.08, H: 4.96, N: 9.50.

\section{1-(2-(4-(3-Methoxyphenyl)piperazin-1-yl)-2-oxo-1-phenylethyl)pyrrolidine-2,5-dione}

(24). White solid. Yield: $73 \%(1.78 \mathrm{~g})$; mp. $161.4-162.7^{\circ} \mathrm{C}$; TLC: $\mathrm{R}_{\mathrm{f}}=0.36\left(\mathrm{~S}_{2}\right)$; UPLC (purity $100 \%): t_{\mathrm{R}}=5.87 \mathrm{~min}$. LC-MS (ESI): $\mathrm{m} / z$ calcd for $\mathrm{C}_{23} \mathrm{H}_{25} \mathrm{~N}_{3} \mathrm{O}_{4}(\mathrm{M}+\mathrm{H})^{+} 408.18$, found 408.2. ${ }^{1} \mathrm{H}$ NMR $\left(300 \mathrm{MHz}, \mathrm{CDCl}_{3}\right) \delta 2.63-2.75(\mathrm{~m}, 5 \mathrm{H}$; 4H, imide, $1 \mathrm{H}$, piperazine), 2.92-3.11 (m, $3 \mathrm{H}$, piperazine $), 3.19-3.33(\mathrm{~m}, 2 \mathrm{H}$, piperazine $), 3.61-3.72(\mathrm{~m}, 1 \mathrm{H}$, piperazine $), 3.73(\mathrm{~s}, 3 \mathrm{H}$, $\left.\mathrm{OCH}_{3}\right), 3.87-4.00(\mathrm{~m}, 1 \mathrm{H}$, piperazine), 6.09 (s, 1H, C $\underline{\mathrm{HCO}}), 6.34$ (t, $\left.1 \mathrm{H}, J=2.3 \mathrm{~Hz}, \mathrm{ArH}\right), 6.38-$ $6.45(\mathrm{~m}, 2 \mathrm{H}, \mathrm{ArH}), 7.12(\mathrm{t}, 1 \mathrm{H}, J=8.2 \mathrm{~Hz}, \mathrm{ArH}), 7.27-7.44(\mathrm{~m}, 5 \mathrm{H}, \mathrm{ArH}) ;{ }^{13} \mathrm{C} \mathrm{NMR}(75 \mathrm{MHz}$, $\left.\mathrm{CDCl}_{3}\right) \delta 28.0,42.4,45.7,48.8,49.0,55.2,56.7,103.1,105.3,109.2,128.6,128.9,129.8,129.9$, 132.8, 152.0, 160.5, 165.1, 176.5. Anal. calcd for $\mathrm{C}_{23} \mathrm{H}_{25} \mathrm{~N}_{3} \mathrm{O}_{4}$ (407.47): C: 67.80, H: 6.18, N: 10.31; Found C: 67.85, H: 6.20, N: 10.29 .

\section{1-(2-Oxo-1-phenyl-2-(4-(3-(trifluoromethoxy)phenyl)piperazin-1-yl)ethyl)pyrrolidine-}

2,5-dione (25). White solid. Yield: $89 \%(2.46 \mathrm{~g})$; mp. 100.3-101.6 ${ }^{\circ} \mathrm{C}$; TLC: $\mathrm{R}_{\mathrm{f}}=0.42\left(\mathrm{~S}_{2}\right)$; UPLC (purity 100\%): $t_{\mathrm{R}}=7.13 \mathrm{~min}$. LC-MS (ESI): $\mathrm{m} / \mathrm{z}$ calcd for $\mathrm{C}_{23} \mathrm{H}_{22} \mathrm{~F}_{3} \mathrm{~N}_{3} \mathrm{O}_{4}(\mathrm{M}+\mathrm{H})^{+} 462.16$, found 462.2. ${ }^{1} \mathrm{H} \mathrm{NMR}\left(300 \mathrm{MHz}, \mathrm{CDCl}_{3}\right) \delta 2.63-2.79(\mathrm{~m}, 5 \mathrm{H} ; 4 \mathrm{H}$, imide, $1 \mathrm{H}$, piperazine), $3.00-3.16(\mathrm{~m}, 2 \mathrm{H}$, piperazine $), 3.22-3.39(\mathrm{~m}, 3 \mathrm{H}$, piperazine $), 3.93-4.05(\mathrm{~m}, 1 \mathrm{H}$, piperazine $)$, 3.63-3.75 (m, 1H, piperazine), $6.12(\mathrm{~s}, 1 \mathrm{H}, \mathrm{CHCO}), 6.62(\mathrm{~s}, 1 \mathrm{H}, \mathrm{ArH}), 6.66-6.78(\mathrm{~m}, 2 \mathrm{H}, \mathrm{ArH})$, 7.16-7.28 (m, 1H, ArH), 7.32-7.48 (m, 5H, ArH); ${ }^{13} \mathrm{C}$ NMR $\left(75 \mathrm{MHz}, \mathrm{CDCl}_{3}\right) \delta 28.0,42.2$, $45.5,48.3,48.5,56.8,108.8,112.1,114.2,120,4$ (q, $J=256.8 \mathrm{~Hz}), 128.7,128.9,129.8,130.2$, 
132.8, 150.2, 151.9, 165.1, 176.3. Anal. calcd for $\mathrm{C}_{23} \mathrm{H}_{22} \mathrm{~F}_{3} \mathrm{~N}_{3} \mathrm{O}_{4}$ (461.44): C: 59.87, H: 4.81, $\mathrm{N}: 9.11$; Found C: $59.85, \mathrm{H}: 4.79, \mathrm{~N}: 9.15$.

1-(2-(4-(3-(Methylthio)phenyl)piperazin-1-yl)-2-oxo-1-phenylethyl)pyrrolidine-2,5-dione (26). White solid. Yield: $72 \%$ (1.82 g); m.p. 88.1-89.2 ${ }^{\circ} \mathrm{C}$; TLC: $\mathrm{R}_{\mathrm{f}}=0.69\left(\mathrm{~S}_{3}\right)$; UPLC (purity 98.55\%): $t_{\mathrm{R}}=6.60 \mathrm{~min}$. LC-MS (ESI): $\mathrm{m} / z$ calcd for $\mathrm{C}_{23} \mathrm{H}_{25} \mathrm{~N}_{3} \mathrm{O}_{3} \mathrm{~S}(\mathrm{M}+\mathrm{H})^{+} 424.16$, found 424.1 . ${ }^{1} \mathrm{H} \mathrm{NMR}\left(500 \mathrm{MHz}, \mathrm{CDCl}_{3}\right) \delta 2.43\left(\mathrm{~s}, 3 \mathrm{H}, \mathrm{CH}_{3}\right), 2.64-2.75(\mathrm{~m}, 5 \mathrm{H} ; 4 \mathrm{H}$, imide, $1 \mathrm{H}$, piperazine), 2.97-3.11 (m, 2H, piperazine), 3.22-3.33 (m, 3H, piperazine), 3.63-3.71 (m, $1 \mathrm{H}$, piperazine), 3.98 (ddd, $1 \mathrm{H}, J=13.17,5.73,3.44 \mathrm{~Hz}$, piperazine), 6.11 (s, 1H, $\mathrm{HCO}$ ), 6.60 (dd, $1 \mathrm{H}, J=8.31$, $2.00 \mathrm{~Hz}, \operatorname{ArH}), 6.71(\mathrm{t}, 1 \mathrm{H}, J=2.00 \mathrm{~Hz}, \operatorname{ArH}), 6.75(\mathrm{~d}, 1 \mathrm{H}, J=7.66 \mathrm{~Hz}, \operatorname{ArH}), 7.14(\mathrm{t}, 1 \mathrm{H}, J=8.02$ $\mathrm{Hz}, \mathrm{ArH}), 7.32-7.36$ (m, 3H, ArH) 7.42 (d, 2H, J=6.87 Hz, ArH). ${ }^{13} \mathrm{C}$ NMR $\left(126 \mathrm{MHz}, \mathrm{CDCl}_{3}\right)$ $\delta 15.9,28.1,42.5,45.8,48.9,49.1,56.9,113.6,114.9,118.6,128.8,129.0,129.6,129.9,133.0$, 139.6, 151.1, 165.1, 176.4. Anal. calcd for $\mathrm{C}_{23} \mathrm{H}_{25} \mathrm{~N}_{3} \mathrm{O}_{3} \mathrm{~S}$ (423.53): C: $65.23, \mathrm{H}: 5.95$, N: 9.92; Found C: 65.38, H: 5.87, N: 9.98.

\section{1-(2-Oxo-1-phenyl-2-(4-(3-((trifluoromethyl)thio)phenyl)piperazin-1-}

yl)ethyl)pyrrolidine-2,5-dione (27). White solid. Yield: $64 \%(1.83 \mathrm{~g})$; m.p. $97.8-99.2{ }^{\circ} \mathrm{C}$; TLC: $\mathbf{R}_{\mathrm{f}}=0.48\left(\mathrm{~S}_{3}\right)$; UPLC (purity $100 \%$ ): $t_{\mathrm{R}}=7.55 \mathrm{~min}$. LC-MS (ESI): $\mathrm{m} / \mathrm{z}$ calcd for $\mathrm{C}_{23} \mathrm{H}_{22} \mathrm{~F}_{3} \mathrm{~N}_{3} \mathrm{O}_{3} \mathrm{~S}(\mathrm{M}+\mathrm{H})^{+} 478.13$, found 478.1. ${ }^{1} \mathrm{H} \mathrm{NMR}\left(500 \mathrm{MHz}, \mathrm{CDCl}_{3}\right) \delta$ 2.64-2.78 (m, $5 \mathrm{H}$; $4 \mathrm{H}$, imide, $1 \mathrm{H}$, piperazine), 3.01-3.07 (m, 1H, piperazine), 3.09-3.15 $(\mathrm{m}, 1 \mathrm{H}$, piperazine $)$, 3.24-3.32 (m, 2H, piperazine), $3.34(\mathrm{dd}, 1 \mathrm{H}, J=7.7,3.2 \mathrm{~Hz}$, piperazine), 3.62-3.75 (m, $1 \mathrm{H}$, piperazine), 3.99 (ddd, $1 \mathrm{H}, J=13.2,5.7,3.4 \mathrm{~Hz}$, piperazine), 6.11 (s, 1H, CㅂCO), 6.92 (dd, $1 \mathrm{H}$, $J=8.0,2.3 \mathrm{~Hz}, \operatorname{ArH}), 7.06$ (s, 1H, ArH), $7.12(\mathrm{~d}, 1 \mathrm{H}, J=7.4 \mathrm{~Hz}, \operatorname{ArH}), 7.24-7.29$ (m, 1H, ArH), 7.33-7.38 (m, 3H, ArH), 7.43 (d, 2H, $J=6.8 \mathrm{~Hz}, \mathrm{ArH}) .{ }^{13} \mathrm{C} \mathrm{NMR}\left(126 \mathrm{MHz}, \mathrm{CDCl}_{3}\right) \delta 28.1$, 45.6, 48.4, 48.7, 56.9, 118.5, 123.7, 125.3, 127.8, 128.5, 129.4 (d, J=141.2 Hz), 129.6 (d, $J=$ 137.0 Hz), 130.9, 132.9, 151.4, 165.2, 176.4. Anal. calcd for $\mathrm{C}_{23} \mathrm{H}_{22} \mathrm{~F}_{3} \mathrm{~N}_{3} \mathrm{O}_{3} \mathrm{~S}$ (477.50): $\mathrm{C}$ : 57.85, H: 4.64, N: 8.80; Found C: 57.72, H: 4.72, N: 8.73.

\section{1-(2-(4-(3-(Difluoromethyl)phenyl)piperazin-1-yl)-2-oxo-1-phenylethyl)pyrrolidine-2,5-}

dione (28). White solid. Yield: $83 \%$ (2.13g); m.p. 156.4-157.6 ${ }^{\circ} \mathrm{C}$; TLC: $\mathrm{R}_{\mathrm{f}}=0.55\left(\mathrm{~S}_{2}\right)$; UPLC (purity $100 \%$ ): $t_{\mathrm{R}}=6.36 \mathrm{~min}$. LC-MS (ESI): $\mathrm{m} / z$ calcd for $\mathrm{C}_{23} \mathrm{H}_{23} \mathrm{~F}_{2} \mathrm{~N}_{3} \mathrm{O}_{3}(\mathrm{M}+\mathrm{H})^{+} 428.17$, found 428.2. ${ }^{1} \mathrm{H}$ NMR $\left(500 \mathrm{MHz}, \mathrm{CDCl}_{3}\right) \delta$ 2.68-2.74 (m, 4H, imide), 3.01-3.17 (m, 3H, piperazine), 3.23-3.40 (m, 3H, piperazine), 3.65-3.74 (m, 1H, piperazine), 4.01 (ddd, $1 \mathrm{H}, J=13.17,5.73$, $2.86 \mathrm{~Hz}$, piperazine), $6.12(\mathrm{~s}, 1 \mathrm{H}, \mathrm{C} \underline{\mathrm{HCO}}), 6.55\left(\mathrm{t}, 1 \mathrm{H}, J=56.42 \mathrm{~Hz}, \mathrm{CH}_{2}\right), 6.90-6.94(\mathrm{~m}, 2 \mathrm{H}$, $\mathrm{ArH}), 6.98$ (d, 1H, J=7.45 Hz, ArH), 7.28-7.38 (m, 4H, ArH), 7.42-7.45 (m, 2H, ArH). ${ }^{13} \mathrm{C}$ NMR $\left(126 \mathrm{MHz} \mathrm{CDCl}_{3}\right) \delta 28.1,42.4,45.7,48.8$ (d, $\left.J=29.6 \mathrm{~Hz}\right), 56.9,113.1,116.2(\mathrm{~d}, J=341.0$ $\mathrm{Hz}), 118.6,129.4$ (d, $J=143.1 \mathrm{~Hz}), 129.4$ (d, $J=86.3 \mathrm{~Hz}), 133.0,151.0,162.3,165.2,176.4$. Anal. calcd for $\mathrm{C}_{23} \mathrm{H}_{23} \mathrm{~F}_{2} \mathrm{~N}_{3} \mathrm{O}_{3}$ (427.45): C: 64.63, H: 5.42, N: 9.83; Found C: 64.51, H: 5.28, N: 9.71 .

1-(2-(4-(3-Aminophenyl)piperazin-1-yl)-2-oxo-1-phenylethyl)pyrrolidine-2,5-dione (29). White solid. Yield: $65 \%(1.53 \mathrm{~g})$; mp. $122.1-123.6^{\circ} \mathrm{C}$; TLC: $\mathrm{R}_{\mathrm{f}}=0.48\left(\mathrm{~S}_{3}\right)$; UPLC (purity 98.75\%): $t_{\mathrm{R}}=3.32 \mathrm{~min}$. LC-MS (ESI): $\mathrm{m} / z$ calcd for $\mathrm{C}_{22} \mathrm{H}_{24} \mathrm{~N}_{4} \mathrm{O}_{3}(\mathrm{M}+\mathrm{H})^{+} 393.19$, found 393.2. ${ }^{1} \mathrm{H}$ NMR $\left(300 \mathrm{MHz}, 300 \mathrm{MHz}, \mathrm{CDCl}_{3}\right) \delta 2.56-2.81$ (m, 5H; 4H, imide, 1H, piperazine), $2.94-$ $3.10(\mathrm{~m}, 3 \mathrm{H}$, piperazine), 3.17-3.36 (m, 3H, piperazine), 3.52-3.72 (m, $1 \mathrm{H}$, piperazine $), 5.88$ (br. s, 2H, $\mathrm{NH}_{2}$ ), 6.04-6.12 (m, 1H, CㅌC), 6.23 (dt, 1H, J=1.9, 8.3 Hz, ArH), 7.01 (t, 1H, $J=8.0 \mathrm{~Hz}, \mathrm{ArH}), 7.10(\mathrm{t}, 1 \mathrm{H}, J=8.1 \mathrm{~Hz}, \mathrm{ArH}), 7.31-7.69(\mathrm{~m}, 6 \mathrm{H}, \mathrm{ArH}) .{ }^{13} \mathrm{C}$ NMR $(75 \mathrm{MHz}$, $\left.\mathrm{CDCl}_{3}\right) \delta 28.0,28.2,31.0,42.4,45.8,48.6,48.9,49.1,56.8,59.3,103.3,107.1,107.7,112.6$, 128.6, 128.7, 128.8, 128.9, 129.5, 129.7, 129.8, 129.9, 130.0, 132.9, 133.7, 147.4, 151.3, 151.9, 164.7, 165.0, 176.3, 176.5. Anal. calcd for $\mathrm{C}_{22} \mathrm{H}_{24} \mathrm{~N}_{4} \mathrm{O}_{3}$ (392.45): C: 67.33, H: 6.16, N: 14.28; Found C: $67.30, \mathrm{H}: 6.15, \mathrm{~N}: 14.31$. 
1-(2-(4-(3-Isopropylphenyl)piperazin-1-yl)-2-oxo-1-phenylethyl)pyrrolidine-2,5-dione (30). White solid. Yield: $72 \%$ (1.81g); mp. $149.4-151.2^{\circ} \mathrm{C}$; TLC: $\mathrm{R}_{\mathrm{f}}=0.46\left(\mathrm{~S}_{2}\right)$; UPLC (purity $100 \%): t_{\mathrm{R}}=7.19 \mathrm{~min}$. LC-MS (ESI): $\mathrm{m} / z$ calcd for $\mathrm{C}_{25} \mathrm{H}_{29} \mathrm{~N}_{3} \mathrm{O}_{3}(\mathrm{M}+\mathrm{H})^{+} 420.22$, found 420.3 . ${ }^{1} \mathrm{H}$ NMR $\left(300 \mathrm{MHz}, 300 \mathrm{MHz}, \mathrm{CDCl}_{3}\right) \delta 1.09-1.35\left(\mathrm{~m}, 6 \mathrm{H}, \mathrm{CH}\left(\mathrm{CH}_{3}\right)_{2}\right), 2.15$ (s, 4H, imide), 2.52-4.10 (m, 9H; $1 \mathrm{H}, \mathrm{C} \underline{\mathrm{H}}\left(\mathrm{CH}_{3}\right)_{2} ; 8 \mathrm{H}$, piperazine), 6.12 (s, $\left.1 \mathrm{H}, \mathrm{C} \underline{\mathrm{HCO}}\right), 6.57-8.15(\mathrm{~m}, 9 \mathrm{H}$, ArH). ${ }^{13} \mathrm{C}$ NMR $\left(75 \mathrm{MHz}, \mathrm{CDCl}_{3}\right) \delta 22.6,22.7,24.0,28.1,29.7,30.5,30.9,33.9,42.9,46.3$, 50.8, 51.1, 51.3, 56.7, 118.0, 118.7, 122.2, 125.8, 127.1, 128.7, 128.8, 129.2, 129.5, 129.8, 129.8, 130.4, 132.9, 148.8, 165.2, 176.5, 207.2. Anal. calcd for $\mathrm{C}_{25} \mathrm{H}_{29} \mathrm{~N}_{3} \mathrm{O}_{3}$ (419.53): C: 71.57, H: 6.97, N: 10.02; Found C: 71.60, H: 6.99, N: 10.04 .

\section{1-(2-(4-(3-(tert-Butyl)phenyl)piperazin-1-yl)-2-oxo-1-phenylethyl)pyrrolidine-2,5-dione}

(31). White solid. Yield: $64 \%$ (1.66 g); mp. $158.2-160.7^{\circ} \mathrm{C}$; TLC: $\mathrm{R}_{\mathrm{f}}=0.42\left(\mathrm{~S}_{1}\right)$; UPLC (purity 97.92\%): $t_{\mathrm{R}}=7.63 \mathrm{~min}$. LC-MS (ESI): $\mathrm{m} / z$ calcd for $\mathrm{C}_{26} \mathrm{H}_{31} \mathrm{~N}_{3} \mathrm{O}_{3}(\mathrm{M}+\mathrm{H})^{+} 434.24$, found 434.2. ${ }^{1} \mathrm{H}$ NMR $\left(300 \mathrm{MHz}, \mathrm{CDCl}_{3}\right) \delta 1.24-1.35\left(\mathrm{~m}, 9 \mathrm{H}, \mathrm{C}\left(\mathrm{C}_{3}\right)_{3}\right), 2.71(\mathrm{~s}, 4 \mathrm{H}$, imide), 2.87-4.58 (m, $8 \mathrm{H}$, piperazine), $6.08(\mathrm{~s}, 1 \mathrm{H}, \mathrm{CHCO}), 7.29-7.56(\mathrm{~m}, 8 \mathrm{H}, \mathrm{ArH}), 7.79$ (br. s, $1 \mathrm{H}, \mathrm{ArH}) ;{ }^{13} \mathrm{C} \mathrm{NMR}$ $\left(75 \mathrm{MHz}, \mathrm{CDCl}_{3}\right) \delta 28.1,31.1,35.2,39.9,43.2,54.5,56.5,117.6,118.1,129.0,129.3,129.8$, 130.1, 154.5, 165.3, 176.4. Anal. calcd for $\mathrm{C}_{26} \mathrm{H}_{31} \mathrm{~N}_{3} \mathrm{O}_{3}$ (433.55): C: 72.03, H: 7.21, N: 9.69; Found C: 72.10, H: 7.20, N: 9.63.

\section{1-(2-(4-([1,1'-Biphenyl]-3-yl)piperazin-1-yl)-2-oxo-1-phenylethyl)pyrrolidine-2,5-dione}

(32). White solid. Yield: $82 \%$ (2.23 g); mp. 114.1-115.4 ${ }^{\circ} \mathrm{C}$; TLC: $\mathrm{R}_{\mathrm{f}}=0.4\left(\mathrm{~S}_{3}\right)$; UPLC (purity $100 \%): t_{\mathrm{R}}=7.40 \mathrm{~min}$. LC-MS (ESI): $\mathrm{m} / z$ calcd for $\mathrm{C}_{28} \mathrm{H}_{27} \mathrm{~N}_{3} \mathrm{O}_{3}(\mathrm{M}+\mathrm{H})^{+} 454.20$, found 454.5 . ${ }^{1} \mathrm{H}$ NMR $\left(300 \mathrm{MHz}, \mathrm{CDCl}_{3}\right) \delta 2.56-2.81(\mathrm{~m}, 5 \mathrm{H} ; 4 \mathrm{H}$, imide, $1 \mathrm{H}$, piperazine), 3.00-3.21 (m, $2 \mathrm{H}$, piperazine), 3.23-3.56 (m, 3H, piperazine), 3.65-3.79 (m, 1H, piperazine), 3.94-4.11 (m, 1H, piperazine), 6.14 (s, 1H, C $\underline{H C O}), 6.83$ (dd, $1 \mathrm{H}, J=8.1,2.0 \mathrm{~Hz}, \mathrm{ArH}), 7.00-7.17$ (m, 2H, $\mathrm{ArH}), 7.27-7.62(\mathrm{~m}, 11 \mathrm{H}, \mathrm{ArH}) ;{ }^{13} \mathrm{C}$ NMR $\left(75 \mathrm{MHz}, \mathrm{CDCl}_{3}\right) \delta 28.1,42.5,45.8,49.0,49.2$, 56.8, 115.4, 115.6, 119.7, 127.2, 127.4, 128.7, 128.8, 129.6, 129.9, 132.9, 141.4, 142.5, 151.1, 165.0, 176.4. Anal. calcd for $\mathrm{C}_{28} \mathrm{H}_{27} \mathrm{~N}_{3} \mathrm{O}_{3}$ (453.54): C: 74.15, H: 6.00, N: 9.27; Found C: 74.12, H: 6.03, N: 9.25.

\section{1-(2-(4-(3-(Benzyloxy)phenyl)piperazin-1-yl)-2-oxo-1-phenylethyl)pyrrolidine-2,5-dione}

(33). White solid. Yield: $64 \%(1.86 \mathrm{~g})$; mp. 94.1-95.6 $6^{\circ}$; TLC: $\mathrm{R}_{\mathrm{f}}=0.49\left(\mathrm{~S}_{2}\right)$; UPLC (purity $100 \%): t_{\mathrm{R}}=7.43 \mathrm{~min}$. LC-MS (ESI): $\mathrm{m} / z$ calcd for $\mathrm{C}_{29} \mathrm{H}_{29} \mathrm{~N}_{3} \mathrm{O}_{4}(\mathrm{M}+\mathrm{H})^{+} 484.21$, found 484.2. ${ }^{1} \mathrm{H}$ NMR $\left(300 \mathrm{MHz}, \mathrm{CDCl}_{3}\right) \delta 2.59-2.88(\mathrm{~m}, 5 \mathrm{H}$; 4H, imide, $1 \mathrm{H}$, piperazine), 3.03-3.22 (m, $2 \mathrm{H}$, piperazine), $3.25-3.55(\mathrm{~m}, 3 \mathrm{H}$, piperazine $), 3.62-3.75(\mathrm{~m}, 1 \mathrm{H}$, piperazine $), 3.90-4.08(\mathrm{~m}$, $1 \mathrm{H}$, piperazine), 5.12 (s, $\left.2 \mathrm{H},-\mathrm{C}_{2}-\mathrm{O}-\right), 6.14$ (s, 1H, $\left.\mathrm{CHCO}\right), 6.81-6.90$ (m, 1H, ArH), 7.19$7.24(\mathrm{~m}, 2 \mathrm{H}, \mathrm{ArH}), 7.26-7.63(\mathrm{~m}, 11 \mathrm{H}, \mathrm{ArH})$. Anal. calcd for $\mathrm{C}_{29} \mathrm{H}_{29} \mathrm{~N}_{3} \mathrm{O}_{4}$ (483.56): C: 72.03 , H: 6.05, N: 8.69; Found C: 72.05, H: 6.07, N: 8.66.

\section{1-(1-(3-Fluorophenyl)-2-oxo-2-(4-(3-(trifluoromethyl)phenyl)piperazin-1-}

yl)ethyl)pyrrolidine-2,5-dione (34). White solid. Yield: $81 \%(2.25 \mathrm{~g})$; mp. $134.7-136.1^{\circ} \mathrm{C}$; TLC: $\mathrm{R}_{\mathrm{f}}=0.62\left(\mathrm{~S}_{3}\right)$; UPLC (purity $100 \%$ ): $t_{\mathrm{R}}=7.09 \mathrm{~min}$. LC-MS (ESI): $\mathrm{m} / \mathrm{z}$ calcd for $\mathrm{C}_{23} \mathrm{H}_{21} \mathrm{~F}_{4} \mathrm{~N}_{3} \mathrm{O}_{3}(\mathrm{M}+\mathrm{H})^{+}$464.15, found 464.0. ${ }^{1} \mathrm{H}$ NMR $\left(300 \mathrm{MHz}, \mathrm{CDCl}_{3}\right) \delta 2.58-2.83(\mathrm{~m}, 5 \mathrm{H}$; $4 \mathrm{H}$, imide, $1 \mathrm{H}$, piperazine), $3.00-3.20(\mathrm{~m}, 2 \mathrm{H}$, piperazine), $3.22-3.44(\mathrm{~m}, 3 \mathrm{H}$, piperazine), 3.64-3.80 (m, 1H, piperazine), 3.90-4.04 (m, 1H, piperazine), 6.09 (s, 1H CㅂCO), 6.93-7.15 (m, 5H, ArH), 7.29-7.38 (m, 1H, ArH), 7.39-7.48 (m, 2H, ArH). ${ }^{13} \mathrm{C} \mathrm{NMR}\left(75 \mathrm{MHz}, \mathrm{CDCl}_{3}\right)$ $\delta$ 28.0, 28.1, 42.3, 45.6, 48.5, 48.6, 56.0, 112.7 (q, $J=3.4 \mathrm{~Hz}), 115.6,115.9,116.8$ (q, J=3.4 Hz), 119.3, 124.1 (q, $J=271.8 \mathrm{~Hz}), 128.7$ (d, $J=3.4 \mathrm{~Hz}), 129.7,131.5$ (q, $J=32.2 \mathrm{~Hz}), 131.7,132.8$, 150.7, 161.1, 164.4, 164.9, 176.3. ${ }^{19} \mathrm{~F}$ NMR $\left(282 \mathrm{MHz}, \mathrm{CDCl}_{3}\right) \delta-111.83--111.78(\mathrm{~m}, 1 \mathrm{~F}),-$ 62.78 (br. s, 3F). Anal. calcd for $\mathrm{C}_{23} \mathrm{H}_{21} \mathrm{~F}_{4} \mathrm{~N}_{3} \mathrm{O}_{3}$ (463.43): C: 59.61, $\mathrm{H}: 4.57, \mathrm{~N}: 9.07$; Found C: 59.58, H: 4.55, N: 9.05 . 
1-(1-(4-Fluorophenyl)-2-oxo-2-(4-(3-(trifluoromethyl)phenyl)piperazin-1-

yl)ethyl)pyrrolidine-2,5-dione (35). White solid. Yield: $73 \%(2.03 \mathrm{~g})$; mp. 88.8-90.7 ${ }^{\circ} \mathrm{C}$; TLC: $\mathrm{R}_{\mathrm{f}}=0.63\left(\mathrm{~S}_{3}\right)$; UPLC (purity $100 \%$ ): $t_{\mathrm{R}}=7.07 \mathrm{~min}$. LC-MS (ESI): $\mathrm{m} / z$ calcd for $\mathrm{C}_{23} \mathrm{H}_{21} \mathrm{~F}_{4} \mathrm{~N}_{3} \mathrm{O}_{3}$ $(\mathrm{M}+\mathrm{H})^{+}$464.15, found 464.1. ${ }^{1} \mathrm{H}$ NMR $\left(300 \mathrm{MHz}, \mathrm{CDCl}_{3}\right) \delta 2.61-2.89(\mathrm{~m}, 5 \mathrm{H} ; 4 \mathrm{H}$, imide, $1 \mathrm{H}$, piperazine $), 3.02-3.46(\mathrm{~m}, 5 \mathrm{H}$, piperazine $), 3.67-3.80(\mathrm{~m}, 1 \mathrm{H}$, piperazine $), 3.88-4.04(\mathrm{~m}, 1 \mathrm{H}$, piperazine), 6.09 (s, 1H CㅌCO), 6.94-7.27 (m, 6H, ArH), 7.29-7.40 (m, 2H, ArH). ${ }^{13} \mathrm{C} \mathrm{NMR}$ $\left(75 \mathrm{MHz}, \mathrm{CDCl}_{3}\right) \delta 28.0,42.3,45.6,48.5,48.6,56.0,112.7$ (q, J=3.4 Hz), 115.9, 116.2, 116.7 (q, $J=3.4 \mathrm{~Hz}), 117.0,119.3,124.1$ (q, $J=272.9 \mathrm{~Hz}), 125.5$ (d, $J=3.4 \mathrm{~Hz}), 129.7,130.2,130.3$, 131.5 (q, $J=31.1 \mathrm{~Hz}), 135.1$ (d, $J=6.91 \mathrm{~Hz}), 150.7,160.9,164.2,164.5,176.23 .{ }^{19} \mathrm{~F}$ NMR $(282$ $\left.\mathrm{MHz}, \mathrm{CDCl}_{3}\right) \delta-111.73--111.65(\mathrm{~m}, 1 \mathrm{~F}),-62.77$ (br. s, 3F). Anal. calcd for $\mathrm{C}_{23} \mathrm{H}_{21} \mathrm{~F}_{4} \mathrm{~N}_{3} \mathrm{O}_{3}$ (463.43): C: 59.61, H: 4.57, N: 9.07; Found C: 59.61, H: 4.60, N: 9.09.

\section{1-(1-(3,4-Difluorophenyl)-2-oxo-2-(4-(3-(trifluoromethyl)phenyl)piperazin-1-}

yl)ethyl)pyrrolidine-2,5-dione (36). White solid. Yield: $85 \%$ (2.45 g); mp. 145.6-146. ${ }^{\circ} \mathrm{C}$; TLC: $\mathrm{R}_{\mathrm{f}}=0.63\left(\mathrm{~S}_{3}\right)$; UPLC (purity 100\%): $t_{\mathrm{R}}=7.24 \mathrm{~min}$. LC-MS (ESI): $\mathrm{m} / \mathrm{z}$ calcd for $\mathrm{C}_{23} \mathrm{H}_{20} \mathrm{~F}_{5} \mathrm{~N}_{3} \mathrm{O}_{3}(\mathrm{M}+\mathrm{H})^{+} 482.15$, found 482.1. ${ }^{1} \mathrm{H}$ NMR $\left(300 \mathrm{MHz}, \mathrm{CDCl}_{3}\right) \delta 2.68-2.93(\mathrm{~m}, 5 \mathrm{H}$; $4 \mathrm{H}$, imide, $1 \mathrm{H}$, piperazine), 3.03-3.47 ( $\mathrm{m}, 5 \mathrm{H}$, piperazine), 3.68-3.82 (m, 1H, piperazine), 3.85-4.00 (m, 1H, piperazine), 6.04 (s, 1H, C트), 6.94-7.07 (m, 2H, ArH), 7.09-7.22 (m, $3 \mathrm{H}, \mathrm{ArH}), 7.25-7.43$ (m, 2H, ArH); ${ }^{13} \mathrm{C} \mathrm{NMR}\left(75 \mathrm{MHz}, \mathrm{CDCl}_{3}\right) \delta$ 28.0, 42.4, 45.6, 48.6, 55.5, 112.8 (q, $J=3.5 \mathrm{~Hz}), 116.9$ (q, $J=3.5 \mathrm{~Hz}), 117.4,117.7,118.7,119.0,119.2,119.3,122.3,125.9$, 126.2 (dd, $J=5.8,3.5 \mathrm{~Hz}), 129.5,129.8,129.9,131.6$ (q, $J=32.2 \mathrm{~Hz}), 148.6$ (dd, $J=31.1,15.0$ Hz) $150.7,152.0(\mathrm{dd}, J=32.8,14.4 \mathrm{~Hz}), 164.3,176.2 .{ }^{19} \mathrm{~F}$ NMR $\left(282 \mathrm{MHz}, \mathrm{CDCl}_{3}\right) \delta-136.47-$ -135.80 (m, 2F) -62.77 (br. s, 3F). Anal. calcd for $\mathrm{C}_{23} \mathrm{H}_{20} \mathrm{~F}_{5} \mathrm{~N}_{3} \mathrm{O}_{3}$ (481.42): C: 57.38, H: 4.19, N: 8.73; Found C: 57.40, H: 4.17, N: 8.71.

1-(1-Oxo-3-phenyl-1-(4-phenylpiperazin-1-yl)propan-2-yl)pyrrolidine-2,5-dione (37). White solid. Yield: $87 \%$ (2.04 g); mp. $121.7-123.2^{\circ} \mathrm{C}$; TLC: $\mathrm{R}_{\mathrm{f}}=0.62\left(\mathrm{~S}_{3}\right)$; UPLC (purity $100 \%$ ): $t_{\mathrm{R}}=6.19 \mathrm{~min}$. LC-MS (ESI): $\mathrm{m} / z$ calcd for $\mathrm{C}_{23} \mathrm{H}_{25} \mathrm{~N}_{3} \mathrm{O}_{3}(\mathrm{M}+\mathrm{H})^{+} 392.19$, found 392.4. ${ }^{1} \mathrm{H} \mathrm{NMR}$ (300 MHz, $\left.\mathrm{CDCl}_{3}\right) \delta 2.49-2.61$ (m, 4H, imide), 2.95-3.20 (m, 4H, piperazine), 3.31-3.89 (m, $6 \mathrm{H}$, piperazine, $-\mathrm{CH}_{2}-\underline{ }^{-}, 5.19$ (dd, $\left.1 \mathrm{H}, J=10.3,6.2 \mathrm{~Hz}, \mathrm{CHCO}\right), 6.83-6.96(\mathrm{~m}, 3 \mathrm{H}, \mathrm{ArH}), 7.13-$ $7.33(\mathrm{~m}, 7 \mathrm{H}, \mathrm{ArH}) ;{ }^{13} \mathrm{C} \mathrm{NMR}\left(75 \mathrm{MHz}, \mathrm{CDCl}_{3}\right) \delta 27.8,34.2,42.5,45.4,49.3,49.6,52.9,116.6$, 120.7, 127.1, 128.6, 129.1, 129.3, 136.7, 150.7, 166.4, 176.5. Anal. calcd for $\mathrm{C}_{23} \mathrm{H}_{25} \mathrm{~N}_{3} \mathrm{O}_{3}$ (391.46): C: 70.57, H: 6.44, N: 10.73; Found C: 70.60, H: 6.42, N: 10.71.

\section{1-(1-(4-(3-Chlorophenyl)piperazin-1-yl)-1-oxo-3-phenylpropan-2-yl)pyrrolidine-2,5-}

dione (38). White solid. Yield: $87 \%(2.21 \mathrm{~g})$; mp. $114.3-116.2^{\circ} \mathrm{C}$; TLC: $\mathrm{R}_{\mathrm{f}}=0.8\left(\mathrm{~S}_{3}\right)$; UPLC (purity $100 \%$ ): $t_{\mathrm{R}}=6.95 \mathrm{~min}$. LC-MS (ESI): $\mathrm{m} / z$ calcd for $\mathrm{C}_{23} \mathrm{H}_{24} \mathrm{ClN}_{3} \mathrm{O}_{3}(\mathrm{M}+\mathrm{H})^{+} 426.15$, found 426.3. ${ }^{1} \mathrm{H}$ NMR (300 MHz, $\left.\mathrm{CDCl}_{3}\right)$ 2.49-2.64 (m, 4H, imide), 2.95-3.21 (m, 4H, piperazine), 3.30-3.86 (m, 6H; 4H, piperazine, $2 \mathrm{H},-\underline{\mathrm{C}}_{2}-$ ), 5.17 (dd, $\left.1 \mathrm{H}, J=10.0,6.2 \mathrm{~Hz}, \mathrm{C} \underline{\mathrm{HCO}}\right), 6.73$ (ddd, $1 \mathrm{H}, J=8.3,2.2,0.9 \mathrm{~Hz}, \mathrm{ArH}), 6.79-6.88$ (m, 2H, ArH), 7.06-7.35 (m, 6H, ArH); ${ }^{13} \mathrm{C} \mathrm{NMR}$ $\left(75 \mathrm{MHz}, \mathrm{CDCl}_{3}\right) \delta 27.8,34.2,42.2,45.2,48.7,49.0,52.9,114.4,116.3,120.2,127.1,128.6$, 129.1, 130.2, 135.0, 136.6, 151.7, 166.4, 176.5. Anal. calcd for $\mathrm{C}_{23} \mathrm{H}_{24} \mathrm{ClN}_{3} \mathrm{O}_{3}$ (425.91): C: 64.86, H: 5.68, N: 9.87; Found C: 64.83, H: 5.70, N: 9.86.

1-(1-Oxo-3-phenyl-1-(4-(m-tolyl)piperazin-1-yl)propan-2-yl)pyrrolidine-2,5-dione (39). White solid. Yield: $75 \%(1.82 \mathrm{~g}) ; 123.8-125.1^{\circ} \mathrm{C}$; TLC: $\mathrm{R}_{\mathrm{f}}=0.65\left(\mathrm{~S}_{3}\right)$; UPLC (purity 100\%): $t_{\mathrm{R}}$ $=6.62$ min. LC-MS (ESI): $\mathrm{m} / z$ calcd for $\mathrm{C}_{24} \mathrm{H}_{27} \mathrm{~N}_{3} \mathrm{O}_{3}(\mathrm{M}+\mathrm{H})^{+} 406.2$, found 406.3. ${ }^{1} \mathrm{H} \mathrm{NMR}$ $\left(300 \mathrm{MHz}, \mathrm{CDCl}_{3}\right) \delta 2.31\left(\mathrm{~s}, 3 \mathrm{H}, \mathrm{CH}_{3}\right), 2.46-2.65(\mathrm{~m}, 4 \mathrm{H}$, imide), 2.97-3.19 (m, 4H, piperazine), 3.30-3.86 (m, 6H; 4H, piperazine; $\left.2 \mathrm{H},-\mathrm{C}_{2}{ }_{2}\right), 5.19(\mathrm{dd}, 1 \mathrm{H}, J=10.2,6.0 \mathrm{~Hz}$, C $\underline{H C O}), 6.65-6.76(\mathrm{~m}, 3 \mathrm{H}, \mathrm{ArH}), 7.11-7.32(\mathrm{~m}, 6 \mathrm{H}, \mathrm{ArH}) .{ }^{13} \mathrm{C} \mathrm{NMR}\left(75 \mathrm{MHz}, \mathrm{CDCl}_{3}\right) \delta 27.8$, 34.2, 42.5, 45.4, 49.4, 49.7, 53.0, 115.5, 115.7, 119.7, 127.1, 127.2, 127.4, 128.6, 128.7, 129.1, 129.6, 136.7, 141.4, 142.5, 151.1, 166.4, 176.5 Anal. calcd for $\mathrm{C}_{24} \mathrm{H}_{27} \mathrm{~N}_{3} \mathrm{O}_{3}$ (405.49): C: 71.09 , H: 6.71, N: 10.36; Found C: 71.12, H: 6.73, N: 10.40 . 
1-(1-Oxo-3-phenyl-1-(4-(3-(trifluoromethyl)phenyl)piperazin-1-yl)propan-2-

yl)pyrrolidine-2,5-dione (40). White solid. Yield: $84 \%$ (2.31 g); mp. 126.1-127.2 ${ }^{\circ} \mathrm{C}$; TLC: $\mathrm{R}_{\mathrm{f}}$ $=0.72\left(\mathrm{~S}_{3}\right)$; UPLC (purity 100\%): $t_{\mathrm{R}}=7.19 \mathrm{~min}$. LC-MS $(\mathrm{ESI}): \mathrm{m} / z$ calcd for $\mathrm{C}_{24} \mathrm{H}_{24} \mathrm{~F}_{3} \mathrm{~N}_{3} \mathrm{O}_{3}$ $(\mathrm{M}+\mathrm{H})^{+} 460.18$, found $460.3 .{ }^{1} \mathrm{H}$ NMR $\left(300 \mathrm{MHz}, \mathrm{CDCl}_{3}\right) \delta 2.44-2.64$ (m, 4H, imide), 3.00$3.24\left(\mathrm{~m}, 4 \mathrm{H}\right.$, piperazine), 3.32-3.89 (m, 6H; 4H, piperazine, $\left.2 \mathrm{H}, \mathrm{CH}_{2}-\mathrm{ArH}\right), 5.18(\mathrm{dd}, 1 \mathrm{H}$, $J=10.0,6.2 \mathrm{~Hz}, \mathrm{C} \underline{\mathrm{HCO}}), 6.94-7.42(\mathrm{~m}, 9 \mathrm{H}, \mathrm{ArH}) ;{ }^{13} \mathrm{C} \mathrm{NMR}\left(75 \mathrm{MHz}, \mathrm{CDCl}_{3}\right) \delta 27.8,34.2$, 42.2, 45.2, 48.7, 49.0, 52.9, 112.7 (q, $J=3.4 \mathrm{~Hz}), 116.8$ (q, $J=3.4 \mathrm{~Hz}), 124.1$ (q, $J=271.8 \mathrm{~Hz})$, 127.1, 128.6, 129.1, 129.7, 131.5 (q, $J=32.2 \mathrm{~Hz}), 136.6,150.8,166,5,176.5 ;{ }^{19} \mathrm{~F}$ NMR $(282$ $\left.\mathrm{MHz}, \mathrm{CDCl}_{3}\right) \delta-62.76$ (br. s, 3F). Anal. calcd for $\mathrm{C}_{24} \mathrm{H}_{24} \mathrm{~F}_{3} \mathrm{~N}_{3} \mathrm{O}_{3}$ (459.47): C: 62.74, H: 5.27, N: 9.15; Found C: 62.72, H: 5.25, N: 9.13.

1-(1-Oxo-3-phenyl-1-(4-(3-(trifluoromethoxy)phenyl)piperazin-1-yl)propan-2-

yl)pyrrolidine-2,5-dione (41). White solid. Yield: 83\% (2.36 g); mp. 104.4-105.5 C; TLC: $\mathrm{R}_{\mathrm{f}}$ $=0.71\left(\mathrm{~S}_{3}\right)$; UPLC (purity 100\%): $t_{\mathrm{R}}=7.37 \mathrm{~min}$. LC-MS (ESI): $\mathrm{m} / z$ calcd for $\mathrm{C}_{24} \mathrm{H}_{24} \mathrm{~F}_{3} \mathrm{~N}_{3} \mathrm{O}_{4}$ $(\mathrm{M}+\mathrm{H})^{+} 476.17$, found $476.3 .{ }^{1} \mathrm{H}$ NMR $\left(300 \mathrm{MHz}, \mathrm{CDCl}_{3}\right) \delta 2.41-2.73(\mathrm{~m}, 4 \mathrm{H}$, imide), 2.94 $3.22\left(\mathrm{~m}, 4 \mathrm{H}\right.$, piperazine), $3.31-3.86\left(\mathrm{~m}, 6 \mathrm{H} ; 4 \mathrm{H}\right.$, piperazine, $\left.2 \mathrm{H},-\mathrm{CH}_{2}-\right), 5.18(\mathrm{dd}, 1 \mathrm{H}, J=10.0$, $6.2 \mathrm{~Hz}, \mathrm{C} \underline{\mathrm{HCO}}), 6.62-6.85$ (m, 3H, ArH), 7.11-7.36 (m, 6H, ArH); ${ }^{13} \mathrm{C} \mathrm{NMR}\left(75 \mathrm{MHz}, \mathrm{CDCl}_{3}\right)$ $\delta$ 27.8, 34.2, 42.2, 45.2, 48.6, 48.9, 52.9, 108.8, 112.2, 114,2, 120.4 (q, J=256.8 Hz), 127.1, 128.6, 129.1, 130.2, 136.6, 150.2, 151.9, 166.5, 176.5; ${ }^{19} \mathrm{~F}$ NMR (282 $\left.\mathrm{MHz}, \mathrm{CDCl}_{3}\right) \delta-57.6$ (br. s, 3F). Anal. calcd for $\mathrm{C}_{24} \mathrm{H}_{24} \mathrm{~F}_{3} \mathrm{~N}_{3} \mathrm{O}_{4}$ (475.47): C: 60.63, H: 5.09, N: 8.84; Found C: 60.61, H: 5.08, N: 8.82.

\section{1-(2-(4-(2,3-Dichlorophenyl)piperazin-1-yl)-2-oxo-1-phenylethyl)pyrrolidine-2,5-dione}

(42). White solid. Yield: $81 \%$ (2.17 g); mp.164.2-165.8 ${ }^{\circ} \mathrm{C}$; TLC: $\mathrm{R}_{\mathrm{f}}=0.33\left(\mathrm{~S}_{2}\right)$; UPLC (purity $100 \%): t_{\mathrm{R}}=7.24 \mathrm{~min}$. LC-MS (ESI): $\mathrm{m} / z$ calcd for $\mathrm{C}_{22} \mathrm{H}_{21} \mathrm{Cl}_{2} \mathrm{~N}_{3} \mathrm{O}_{3}(\mathrm{M}+\mathrm{H})^{+} 446.10$, found 446.4 . ${ }^{1} \mathrm{H}$ NMR $\left(300 \mathrm{MHz}, \mathrm{CDCl}_{3}\right) \delta 2.54-2.89(\mathrm{~m}, 6 \mathrm{H} ; 4 \mathrm{H}$, imide, $2 \mathrm{H}$, piperazine), 2.91-3.11 (m, $2 \mathrm{H}$, piperazine), 3.19-3.30 (m, $1 \mathrm{H}$, piperazine), 3.32-3.45 (m, 1H, piperazine), 3.75-3.99 (m, 2H, piperazine), $6.12(\mathrm{~s}, 1 \mathrm{H}, \mathrm{CHCO}), 6.84(\mathrm{dd}, 1 \mathrm{H}, J=7.5,2.0 \mathrm{~Hz}, \mathrm{ArH}), 7.07-7.20(\mathrm{~m}, 2 \mathrm{H}$, ArH), 7.27-7.48 (m, 5H, ArH); ${ }^{\overline{13}} \mathrm{C} \mathrm{NMR}\left(75 \mathrm{MHz}, \mathrm{CDCl}_{3}\right) \delta$ 28.0, 42.7, 46.1, 50.9, 51.0, 56.8, $118.7,125.2,127.5,127.7,128.6,128.8,129.8,132.9,134.2,150.3,165.1,176.3$. Anal. calcd for $\mathrm{C}_{22} \mathrm{H}_{21} \mathrm{Cl}_{2} \mathrm{~N}_{3} \mathrm{O}_{3}$ (446.33): C: 59.20, H: 4.74, N: 9.41; Found C: 59.15, H: 4.75, N: 9.50.

\section{1-(2-(4-(3,4-Dichlorophenyl)piperazin-1-yl)-2-oxo-1-phenylethyl)pyrrolidine-2,5-dione}

(43). White solid. Yield: $79 \%(2.11 \mathrm{~g})$; mp. 177.1-177.8 $8^{\circ}$; TLC: $\mathrm{R}_{\mathrm{f}}=0.33\left(\mathrm{~S}_{2}\right)$; UPLC (purity $100 \%): t_{\mathrm{R}}=7.19 \mathrm{~min}$. LC-MS (ESI): $\mathrm{m} / z$ calcd for $\mathrm{C}_{22} \mathrm{H}_{21} \mathrm{Cl}_{2} \mathrm{~N}_{3} \mathrm{O}_{3}(\mathrm{M}+\mathrm{H})^{+} 446.10$, found 446.3 . ${ }^{1} \mathrm{H}$ NMR $\left(300 \mathrm{MHz}, \mathrm{CDCl}_{3}\right) \delta 2.59-2.81(\mathrm{~m}, 5 \mathrm{H} ; 4 \mathrm{H}$, imide, $1 \mathrm{H}$, piperazine), 2.89-3.13 (m, $2 \mathrm{H}$, piperazine), 3.15-3.40 (m, 3H, piperazine), 3.60-3.73 (m, 1H, piperazine), 3.91-4.04 (m, 1H, piperazine), 6.10 (s, 1H, CㅂCO), $6.64(\mathrm{dd}, 1 \mathrm{H}, J=8.9,2.9 \mathrm{~Hz}, \mathrm{ArH}), 6.86(\mathrm{~d}, 1 \mathrm{H}, J=2.9$ $\mathrm{Hz}, \mathrm{ArH}), 7.23$ (s, 1H, ArH), 7.29-7.46 (m, 5H, ArH); ${ }^{13} \mathrm{C} \mathrm{NMR}\left(75 \mathrm{MHz}, \mathrm{CDCl}_{3}\right) \delta 28.0,42.1$, 45.4, 48.4, 48.6, 56.7, 115.8, 117.8, 123.1, 128.7, 129.0, 129.8, 130.6, 132.8, 132.9, 150.0, 165.1, 176.3. Anal. calcd for $\mathrm{C}_{22} \mathrm{H}_{21} \mathrm{Cl}_{2} \mathrm{~N}_{3} \mathrm{O}_{3}$ (446.33): C: 59.20, H: 4.74, N: 9.41; Found C: 59.16, H: 4.70, N: 9.51 .

\section{1-(2-(4-(3,5-dichlorophenyl)piperazin-1-yl)-2-oxo-1-phenylethyl)pyrrolidine-2,5-dione}

(44). White solid. Yield: $77 \%$ (2.06 g); m.p. $163.8-165.2^{\circ} \mathrm{C}$; TLC: $\mathrm{R}_{\mathrm{f}}=0.42\left(\mathrm{~S}_{2}\right)$; UPLC (purity $100 \%): t_{R}=7.58 \mathrm{~min}$. LC-MS (ESI): $\mathrm{m} / z$ calcd for $\mathrm{C}_{22} \mathrm{H}_{21} \mathrm{Cl}_{2} \mathrm{~N}_{3} \mathrm{O}_{3}(\mathrm{M}+\mathrm{H})^{+} 446.10$, found 446.1 . ${ }^{1} \mathrm{H}$ NMR $\left(500 \mathrm{MHz}, \mathrm{CDCl}_{3}\right) \delta 2.63-2.78(\mathrm{~m}, 5 \mathrm{H} ; 4 \mathrm{H}$, imide, $1 \mathrm{H}$, piperazine), 2.98-3.13 (m, $2 \mathrm{H}$, piperazine), 3.20-3.36 (m, 3H, piperazine), 3.59-3.68 (m, 1H, piperazine), 3.97-4.00 (m, 1H, piperazine), 6.09 (s, 1H, CㅌCO), 6.64 (d, J=1.7 Hz, 2H, ArH), 6.80 (t, J=1.7 Hz, 1H, ArH), 7.33-7.38 (m, 3H, ArH), $7.42(\mathrm{~d}, J=6.7 \mathrm{~Hz}, 2 \mathrm{H}, \mathrm{ArH}) .{ }^{13} \mathrm{C} \mathrm{NMR}\left(126 \mathrm{MHz}, \mathrm{CDCl}_{3}\right) \delta 28.1$, 42.2, 45.4, 48.0, 48.2, 56.9, 114.4, 119.8, 128.8, 129.1, 129.9, 132.9, 135.6, 152.1, 165.2, 176.4 . Anal. calcd for $\mathrm{C}_{22} \mathrm{H}_{21} \mathrm{Cl}_{2} \mathrm{~N}_{3} \mathrm{O}_{3}$ (446.33) C, 59.20; H, 4.74; N, 9.41; Found C: 59.33, H: 4.59, N: 9.28 . 
1-(2-(4-(3,5-bis(trifluoromethyl)phenyl)piperazin-1-yl)-2-oxo-1-phenylethyl)pyrrolidine2,5-dione (45). White solid. Yield: 69\% (2.12 g); m.p. 228.1-229.4 ${ }^{\circ} \mathrm{C}$; TLC: $\mathrm{R}_{\mathrm{f}}=0.47\left(\mathrm{~S}_{3}\right)$; UPLC (purity 100\%): $t_{R}=6.57 \mathrm{~min}$. LC-MS (ESI): $\mathrm{m} / z$ calcd for $\mathrm{C}_{24} \mathrm{H}_{21} \mathrm{~F}_{6} \mathrm{~N}_{3} \mathrm{O}_{3}(\mathrm{M}+\mathrm{H})^{+}$514.10, found 514.4. ${ }^{1} \mathrm{H}$ NMR $\left(500 \mathrm{MHz}, \mathrm{CDCl}_{3}\right) \delta$ 2.64-2.79 (m, 4H, imide), 3.04-3.15 (m, 3H, piperazine), $3.26-3.42(\mathrm{~m}, 3 \mathrm{H}$, piperazine), 3.65-3.74 (m, $1 \mathrm{H}$, piperazine), 3.99-4.07 $(\mathrm{m}, 1 \mathrm{H}$, piperazine), 6.12 (s, 1H, CHCO), 7.15 (s, 2H, ArH), 7.34-7.38 (m, 4H, ArH), 7.43-7.45 (m, $2 \mathrm{H}, \mathrm{ArH}) .{ }^{13} \mathrm{C} \mathrm{NMR}\left(126 \mathrm{MHz}, \mathrm{CDCl}_{3}\right) \delta 28.1,42.1,45.4,48.0(\mathrm{~d}, J=27.2 \mathrm{~Hz}), 56.9,113.0(\mathrm{~d}$, $J=3.6 \mathrm{~Hz}), 115.2,123.4(\mathrm{q}, J=272.4 \mathrm{~Hz}), 128.9,129.2,129.9,132.6$ (q, $J=32.6 \mathrm{~Hz}), 132.8$, 151.2, 165.3, 176.4. Anal. calcd for $\mathrm{C}_{24} \mathrm{H}_{21} \mathrm{~F}_{6} \mathrm{~N}_{3} \mathrm{O}_{3}$ (513.44) C, 56.14; H, 4.12; N, 8.18; Found C: 56.31, H: 4.25, N: 8.11.

\section{1-(2-(4-(3,5-bis((trifluoromethyl)thio)phenyl)piperazin-1-yl)-2-oxo-1-}

phenylethyl)pyrrolidine-2,5-dione (46). White solid. Yield: $64 \%$ (2.21 g); m.p. 232.1-233.6 ${ }^{\circ} \mathrm{C}$; TLC: $\mathrm{R}_{\mathrm{f}}=0.51\left(\mathrm{~S}_{3}\right)$; UPLC (purity 100\%): $t_{R}=6.50 \mathrm{~min}$. LC-MS (ESI): $\mathrm{m} / z$ calcd for $\mathrm{C}_{24} \mathrm{H}_{21} \mathrm{~F}_{6} \mathrm{~N}_{3} \mathrm{O}_{3} \mathrm{~S}_{2}(\mathrm{M}+\mathrm{H})^{+} 578.21$ found 578.2. ${ }^{1} \mathrm{H}$ NMR $\left(500 \mathrm{MHz}, \mathrm{CDCl}_{3}\right) \delta 2.65-2.75(\mathrm{~m}$, $4 \mathrm{H}$, imide), 3.10-3.32 (m, 4H, piperazine), 3.56-3.75 (m, 3H, piperazine), 3.89-4.03 (m, $1 \mathrm{H}$, piperazine), 6.12 (s, 1H, CㅌCO), 6.64-6.58 (m, 2H, ArH), 6.93-7.12 (m, 2H, ArH), 7.28-7.57 (m, 4H, ArH). ${ }^{13} \mathrm{C}$ NMR (126 MHz, $\left.\mathrm{CDCl}_{3}\right) \delta 28.2,42.2,45.4,48.3(\mathrm{~d}, J=27.1 \mathrm{~Hz}), 56.8,113.0$ (d, $J=3.5 \mathrm{~Hz}), 115.2,124.6$ (q, $J=271.4 \mathrm{~Hz}), 128.7,129.2,129.8,132.7,132.9,151.9,165.1$, 176.5. Anal. calcd for $\mathrm{C}_{24} \mathrm{H}_{21} \mathrm{~F}_{6} \mathrm{~N}_{3} \mathrm{O}_{3} \mathrm{~S}_{2}(577.56) \mathrm{C}$, 49.91; H, 3.67; N, 7.28; Found C: 49.64, H: $3.72, \mathrm{~N}: 7.35$.

\section{1-(2-(4-(3-chloro-5-(trifluoromethyl)phenyl)piperazin-1-yl)-2-oxo-1-}

phenylethyl)pyrrolidine-2,5-dione (47). White solid. Yield: $69 \%$ (1.98 g); mp. $214.8-215.5^{\circ} \mathrm{C}$; TLC: $\mathrm{R}_{\mathrm{f}}=0.43\left(\mathrm{~S}_{3}\right)$; UPLC (purity $100 \%$ ): $t_{\mathrm{R}}=7.71 \mathrm{~min}$. LC-MS (ESI): $\mathrm{m} / \mathrm{z}$ calcd for $\mathrm{C}_{23} \mathrm{H}_{21} \mathrm{ClF}_{3} \mathrm{~N}_{3} \mathrm{O}_{3}(\mathrm{M}+\mathrm{H})^{+}$480.12, found 480.2. ${ }^{1} \mathrm{H} \mathrm{NMR}\left(500 \mathrm{MHz}, \mathrm{CDCl}_{3}\right) \delta$ 2.66-2.76 (m, $5 \mathrm{H}$; $4 \mathrm{H}$, imide, $1 \mathrm{H}$, piperazine), 3.04-3.20 (m, $2 \mathrm{H}$, piperazine), $3.24-3.39(\mathrm{~m}, 3 \mathrm{H}$, piperazine), 3.62-3.71 (m, 1H, piperazine), 3.96-4.06 (m, 1H, piperazine), $6.10(\mathrm{~s}, 1 \mathrm{H}, \mathrm{CHCO}), 6.86-6.93$ (m, 2H, ArH), 7.04 (s, 1H, ArH), 7.33-7.38 (m, 3H, ArH), 7.43 (d, 2H, J=6.75 Hz, ArH). ${ }^{13} \mathrm{C}$ NMR $\left(126 \mathrm{MHz}, \mathrm{CDCl}_{3}\right) \delta 28.1,42.2,45.4,47.9,48.2,56.9,110.8(\mathrm{~d}, J=4.2 \mathrm{~Hz}), 116.5(\mathrm{~d}$, $J=3.6 \mathrm{~Hz}), 118.7,123.4$ (q, $J=273.2 \mathrm{~Hz}), 128.8,129.1,129.9,132.8$ (d, $J=32.6 \mathrm{~Hz}), 132.9$, $135.8,151.7,165.2,176.4$.

1-(2-Oxo-1-phenyl-2-(4-(3-(trifluoromethyl)phenyl)piperidin-1-yl)ethyl)pyrrolidine-2,5dione (48). White solid. Yield: 85\% (2,26 g); mp. 100.1-101.5 ${ }^{\circ} \mathrm{C}$; TLC: $\mathrm{R}_{\mathrm{f}}=0.45\left(\mathrm{~S}_{3}\right)$; UPLC (purity 100\%): $t_{\mathrm{R}}=7.24 \mathrm{~min}$. LC-MS (ESI): $\mathrm{m} / z$ calcd for $\mathrm{C}_{24} \mathrm{H}_{23} \mathrm{~F}_{3} \mathrm{~N}_{2} \mathrm{O}_{3}(\mathrm{M}+\mathrm{H})^{+} 445.17$, found 445.1. ${ }^{1} \mathrm{H}$ NMR (300 MHz, $\left.\mathrm{CDCl}_{3}\right) \delta 1.24-2.04$ (m, 3H, piperidin), 2.64-2.80 (m, 8H; 4H, imide, $4 \mathrm{H}$, piperidin), 2.98-3.13 (m, $1 \mathrm{H}$, piperidin), 3.63 (t, $J=14.4 \mathrm{~Hz}, 1 \mathrm{H}$, piperidin), 4.71$4.95(\mathrm{~m}, 1 \mathrm{H}$, piperidin) $6.14(\mathrm{~d}, 1 \mathrm{H}, J=9.4 \mathrm{~Hz}, \mathrm{CHCO}), 7.31-7.54(\mathrm{~m}, 9 \mathrm{H}, \mathrm{ArH})$; Anal. calcd for $\mathrm{C}_{24} \mathrm{H}_{23} \mathrm{~F}_{3} \mathrm{~N}_{2} \mathrm{O}_{3}$ (444.45): C: 64.86, $\mathrm{H}: 5.22, \mathrm{~N}: 6.30$; Found $\mathrm{C}: 64.77, \mathrm{H}: 5.19, \mathrm{~N}: 6.45$.

1-(2-Oxo-1-phenyl-2-(4-(3-(trifluoromethyl)phenyl)-1,4-diazepan-1-yl)ethyl)pyrrolidine2,5-dione (49). White solid. Yield: $83 \%(2,28 \mathrm{~g})$; mp. 80.5-81.2 ${ }^{\circ} \mathrm{C}$; TLC: $\mathrm{R}_{\mathrm{f}}=0.50\left(\mathrm{~S}_{3}\right)$; UPLC (purity 100\%): $t_{\mathrm{R}}=7.04 \mathrm{~min}$. LC-MS (ESI): $\mathrm{m} / z$ calcd for $\mathrm{C}_{24} \mathrm{H}_{24} \mathrm{~F}_{3} \mathrm{~N}_{3} \mathrm{O}_{3}(\mathrm{M}+\mathrm{H})^{+} 460.18$, found 460.2. ${ }^{1} \mathrm{H}$ NMR $\left(300 \mathrm{MHz}, \mathrm{CDCl}_{3}\right) \delta$ 2.06-2.13 (m, 1H, 1,4-diazepane), 2.63-2.75 (m, 4H, imide), 3.17-3.30 (m, 2H, 1,4-diazepane), 3.32-3.62 (m, 5H, 1,4-diazepane), 3.73-3.90 (m, 2H, 1,4-diazepane), 5.93-6.18 (m, 1H, CHCO), 6.74-6.84 (m, 1H, ArH), 6.93 (t, 1H, J=7.0 Hz, ArH), 7.19-7.35 (m, 5H, ArH), 7.39-7.44 (m, 2H, ArH). Anal. calcd for $\mathrm{C}_{24} \mathrm{H}_{24} \mathrm{~F}_{3} \mathrm{~N}_{3} \mathrm{O}_{3}$ (459.47): C, 62.74; H, 5.27; N, 9.15; Found C: 62.77, H: 5.35, N: 9.04. 
1-(3-(4-(3-(Trifluoromethyl)phenyl)piperazine-1-carbonyl)phenyl)pyrrolidine-2,5-dione (52). White solid. Yield: $60 \%$ (1.55 g); mp. 126.9-128.5 ${ }^{\circ}$; TLC: $\mathrm{R}_{\mathrm{f}}=0.31\left(\mathrm{~S}_{2}\right)$; UPLC (purity $100 \%): t_{\mathrm{R}}=6.27 \mathrm{~min}$. LC-MS (ESI): $\mathrm{m} / z$ calcd for $\mathrm{C}_{22} \mathrm{H}_{20} \mathrm{~F}_{3} \mathrm{~N}_{3} \mathrm{O}_{3}(\mathrm{M}+\mathrm{H})^{+} 432.15$, found 432.2 . ${ }^{1} \mathrm{H}$ NMR (300 MHz, $\mathrm{CDCl}_{3}$ ) $\delta 2.88$ (s, 4H, imide), 3.24 (br. s, 4H, piperazine), 3.50-4.05 (m, 4H, piperazine), 6.99-7.18 (m, 3H, ArH), 7.30-7.46 (m, 3H, ArH), 7.47-7.59 (m, 2H, ArH); ${ }^{19} \mathrm{~F}$ NMR (282 MHz, $\left.\mathrm{CDCl}_{3}\right) \delta-62.70$ (br. s, 3F). Anal. calcd for $\mathrm{C}_{22} \mathrm{H}_{20} \mathrm{~F}_{3} \mathrm{~N}_{3} \mathrm{O}_{3}$ (431.42): C: 61.25, H: 4.67, N: 9.74; Found C: 61.24, H: 4.65, N: 9.76.

\section{1-(4-(4-(3-(Trifluoromethyl)phenyl)piperazine-1-carbonyl)phenyl)pyrrolidine-2,5-dione} (53). White solid. Yield: 82\% (2.12 g); mp. 196.0-197.5 ${ }^{\circ}$; TLC: $\mathrm{R}_{\mathrm{f}}=0.47\left(\mathrm{~S}_{2}\right)$; UPLC (purity 99.40\%): $t_{\mathrm{R}}=6.34 \mathrm{~min}$. LC-MS (ESI): $\mathrm{m} / z$ calcd for $\mathrm{C}_{22} \mathrm{H}_{20} \mathrm{~F}_{3} \mathrm{~N}_{3} \mathrm{O}_{3}(\mathrm{M}+\mathrm{H})^{+} 432.15$, found 432.1. ${ }^{1} \mathrm{H}$ NMR $\left(300 \mathrm{MHz}, \mathrm{CDCl}_{3}\right) \delta 2.83-2.93$ (m, 4H, imide), 3.03-3.38 (m, 4H, piperazine), 3.49-4.05 (m, 4H, piperazine), 7.01-7.19 (m, 3H, ArH), 7.31-7.45 (m, 3H, ArH), 7.49-7.59 $(\mathrm{m}, 2 \mathrm{H}, \mathrm{ArH}) ;{ }^{13} \mathrm{C}$ NMR $\left(75 \mathrm{MHz}, \mathrm{CDCl}_{3}\right) \delta 28.4,49.2,112.8(\mathrm{q}, J=3.4 \mathrm{~Hz}), 116.8(\mathrm{q}, J=3.4$ $\mathrm{Hz}), 119.5,124.1$ (q, $J=272.9 \mathrm{~Hz}), 126.5,128.0,129.7,131.5$ (q, $J=31.1 \mathrm{~Hz}), 133.3,135.4$, 151.0, 169.3, 175.8; ${ }^{19} \mathrm{~F}$ NMR $\left(282 \mathrm{MHz}, \mathrm{CDCl}_{3}\right) \delta-62.72$ (s, 3F). Anal. calcd for $\mathrm{C}_{22} \mathrm{H}_{20} \mathrm{~F}_{3} \mathrm{~N}_{3} \mathrm{O}_{3}$ (431.42): C: 61.25, H: 4.67, N: 9.74; Found C: 61.27, H: 4.69, N: 9.75. 
Table S1. Anticonvulsant activity-MES and $6 \mathrm{~Hz}(32 \mathrm{~mA})$ tests in mice i.p. (dose of 100 $\mathrm{mg} / \mathrm{kg}$ ) - compounds 11-47

\begin{tabular}{|c|c|c|c|c|c|c|c|c|}
\hline \multirow{3}{*}{ Compd } & \multirow{3}{*}{$\mathrm{n}$} & \multirow{3}{*}{$\mathrm{R}$} & \multirow{3}{*}{$\mathrm{R}^{1}$} & \multirow{3}{*}{$\mathrm{R}^{2}$} & \multirow{2}{*}{\multicolumn{2}{|c|}{$\frac{\text { MES test }}{{\text { Pretreatment times }(h)^{\mathrm{a}}}^{-}}$}} & \multirow{2}{*}{\multicolumn{2}{|c|}{$\frac{6 \mathrm{~Hz}(32 \mathrm{~mA}) \text { test }}{\text { Pretreatment time }(\mathrm{h})^{\mathrm{a}}}$}} \\
\hline & & & & & & & & \\
\hline & & & & & 0.5 & 2.0 & 0.5 & 2.0 \\
\hline 11 & 0 & $\mathrm{H}$ & $\mathrm{H}$ & - & $4 / 4$ & $0 / 4$ & $3 / 4$ & $0 / 4$ \\
\hline 12 & 0 & $\mathrm{H}$ & $2-\mathrm{Cl}$ & - & $0 / 4$ & $1 / 4$ & - & - \\
\hline 13 & 0 & $\mathrm{H}$ & $3-\mathrm{Cl}$ & - & $3 / 4$ & $0 / 4$ & $4 / 4$ & $3 / 4$ \\
\hline 14 & 0 & $\mathrm{H}$ & 4-Cl & - & $0 / 4$ & $0 / 4$ & - & - \\
\hline 15 & 0 & $\mathrm{H}$ & $2-\mathrm{F}$ & - & $0 / 4$ & $0 / 4$ & - & - \\
\hline 16 & 0 & $\mathrm{H}$ & $3-\mathrm{F}$ & - & $2 / 4$ & $0 / 4$ & - & - \\
\hline 17 & 0 & $\mathrm{H}$ & $4-\mathrm{F}$ & - & $0 / 4$ & $1 / 4$ & - & - \\
\hline 18 & 0 & $\mathrm{H}$ & $2-\mathrm{CH}_{3}$ & - & $0 / 4$ & $0 / 4$ & - & - \\
\hline 19 & 0 & $\mathrm{H}$ & $3-\mathrm{CH}_{3}$ & - & $3 / 4$ & $3 / 4$ & $4 / 4$ & $1 / 4$ \\
\hline 20 & 0 & $\mathrm{H}$ & $4-\mathrm{CH}_{3}$ & - & $3 / 4$ & $2 / 4$ & $1 / 4$ & $0 / 4$ \\
\hline 21 & 0 & $\mathrm{H}$ & $2-\mathrm{CF}_{3}$ & - & $0 / 4$ & $0 / 4$ & - & - \\
\hline 22 & 0 & $\mathrm{H}$ & $3-\mathrm{CF}_{3}$ & - & $4 / 4$ & $4 / 4$ & $4 / 4$ & $2 / 4$ \\
\hline 23 & 0 & $\mathrm{H}$ & $4-\mathrm{CF}_{3}$ & - & $3 / 4$ & $2 / 4$ & $3 / 4$ & $2 / 4$ \\
\hline 24 & 0 & $\mathrm{H}$ & $3-\mathrm{OCH}_{3}$ & - & $1 / 4$ & $1 / 4$ & - & - \\
\hline 25 & 0 & $\mathrm{H}$ & $3-\mathrm{OCF}_{3}$ & - & $4 / 4$ & $4 / 4$ & $4 / 4$ & $1 / 4$ \\
\hline 26 & 0 & $\mathrm{H}$ & $3-\mathrm{SCH}_{3}$ & - & $1 / 4$ & $1 / 4$ & - & - \\
\hline 27 & 0 & $\mathrm{H}$ & $3-\mathrm{SCF}_{3}$ & - & $4 / 4$ & $4 / 4$ & $4 / 4$ & $2 / 4$ \\
\hline 28 & 0 & $\mathrm{H}$ & $3-\mathrm{CHF}_{2}$ & - & $3 / 4$ & $2 / 4$ & $3 / 4$ & $2 / 4$ \\
\hline 29 & 0 & $\mathrm{H}$ & $3-\mathrm{NH}_{2}$ & - & $0 / 4$ & $0 / 4$ & - & - \\
\hline 30 & 0 & $\mathrm{H}$ & $\mathrm{CH}\left(\mathrm{CH}_{3}\right)_{2}$ & - & $1 / 4$ & $1 / 4$ & - & - \\
\hline 31 & 0 & $\mathrm{H}$ & $\mathrm{C}(\mathrm{CH} 3)_{3}$ & - & $2 / 4$ & $0 / 4$ & - & - \\
\hline 32 & 0 & $\mathrm{H}$ & $\mathrm{C}_{6} \mathrm{H}_{5}$ & - & $4 / 4$ & $3 / 4$ & $4 / 4$ & $1 / 4$ \\
\hline 33 & 0 & $\mathrm{H}$ & $\mathrm{OCH}_{2} \mathrm{C}_{6} \mathrm{H}_{5}$ & - & $1 / 4$ & $0 / 4$ & - & - \\
\hline 34 & 0 & $3-\mathrm{F}$ & $3-\mathrm{CF}_{3}$ & - & $1 / 4$ & $1 / 4$ & - & - \\
\hline 35 & 0 & $4-\mathrm{F}$ & $3-\mathrm{CF}_{3}$ & - & $4 / 4$ & $1 / 4$ & $3 / 4$ & $0 / 4$ \\
\hline 36 & 0 & $3,4-\mathrm{F}$ & $3-\mathrm{CF}_{3}$ & - & $1 / 4$ & $1 / 4$ & - & - \\
\hline 37 & 1 & $\mathrm{H}$ & $\mathrm{H}$ & - & $2 / 4$ & $0 / 4$ & - & - \\
\hline 38 & 1 & $\mathrm{H}$ & $3-\mathrm{Cl}$ & - & $2 / 4$ & $0 / 4$ & - & - \\
\hline 39 & 1 & $\mathrm{H}$ & $3-\mathrm{CH}_{3}$ & - & $2 / 4$ & $0 / 4$ & - & - \\
\hline 40 & 1 & $\mathrm{H}$ & $3-\mathrm{CF}_{3}$ & - & $2 / 4$ & $1 / 4$ & - & - \\
\hline 41 & 1 & $\mathrm{H}$ & $3-\mathrm{OCF}_{3}$ & - & $2 / 4$ & $0 / 4$ & - & - \\
\hline 42 & - & - & $2-\mathrm{Cl}$ & $3-\mathrm{Cl}$ & $0 / 4$ & $0 / 4$ & - & - \\
\hline 43 & - & - & $3-\mathrm{Cl}$ & $3-\mathrm{Cl}$ & $0 / 4$ & $0 / 4$ & - & - \\
\hline 44 & - & - & $3-\mathrm{Cl}$ & $5-\mathrm{Cl}$ & $4 / 4$ & $3 / 4$ & $4 / 4$ & $2 / 4$ \\
\hline 45 & - & - & $3-\mathrm{CF}_{3}$ & $5-\mathrm{CF}_{3}$ & $1 / 4$ & $1 / 4$ & - & - \\
\hline 46 & - & - & $3-\mathrm{SCF}_{3}$ & $5-\mathrm{SCF}_{3}$ & $1 / 4$ & $1 / 4$ & - & - \\
\hline 47 & - & - & $3-\mathrm{Cl}$ & $5-\mathrm{CF}_{3}$ & $1 / 4$ & $1 / 4$ & - & - \\
\hline
\end{tabular}

Ratios where at least one animal was protected have been highlighted in bold for easier data interpretation.

${ }^{a}$ Data indicate: number of mice protected / number of mice tested. The animals were examined at two pretreatment times- 0.5 and $2 \mathrm{~h}$. A dash indicates-not tested. 
Table S2. Anticonvulsant activity-PTZ test in mice i.p. (dose of $100 \mathrm{mg} / \mathrm{kg}$ )

\begin{tabular}{|c|c|c|c|c|c|c|}
\hline \multirow{3}{*}{ Compd } & \multirow{3}{*}{$\mathrm{n}$} & \multirow{3}{*}{$\mathrm{R}$} & \multirow{3}{*}{$\mathrm{R}^{1}$} & \multirow{3}{*}{$\mathrm{R}^{2}$} & \multicolumn{2}{|c|}{ PTZ test } \\
\hline & & & & & \multicolumn{2}{|c|}{ Pretreatment times $(\mathrm{h})^{\mathrm{a}}$} \\
\hline & & & & & 0.5 & 2.0 \\
\hline 11 & 0 & $\mathrm{H}$ & $\mathrm{H}$ & - & $3 / 4$ & $1 / 4$ \\
\hline 13 & 0 & $\mathrm{H}$ & $3-\mathrm{Cl}$ & - & $3 / 4$ & $2 / 4$ \\
\hline 19 & 0 & $\mathrm{H}$ & $3-\mathrm{CH}_{3}$ & - & $3 / 4$ & $1 / 4$ \\
\hline 22 & 0 & $\mathrm{H}$ & $3-\mathrm{CF}_{3}$ & - & $4 / 4$ & $3 / 4$ \\
\hline 23 & 0 & $\mathrm{H}$ & $4-\mathrm{CF}_{3}$ & - & $1 / 4$ & $1 / 4$ \\
\hline 25 & 0 & $\mathrm{H}$ & $3-\mathrm{OCF}_{3}$ & - & $2 / 4$ & $2 / 4$ \\
\hline 27 & 0 & $\mathrm{H}$ & $3-\mathrm{SCF}_{3}$ & - & $0 / 4$ & $0 / 4$ \\
\hline 28 & 0 & $\mathrm{H}$ & $3-\mathrm{CHF}_{2}$ & - & $3 / 4$ & $1 / 4$ \\
\hline 32 & 0 & $\mathrm{H}$ & $\mathrm{C}_{6} \mathrm{H}_{5}$ & - & $2 / 4$ & $2 / 4$ \\
\hline 35 & 0 & $4-\mathrm{F}$ & $3-\mathrm{CF}_{3}$ & - & $1 / 4$ & $1 / 4$ \\
\hline 44 & - & - & $3-\mathrm{Cl}$ & $5-\mathrm{Cl}$ & $3 / 4$ & $1 / 4$ \\
\hline
\end{tabular}

Ratios where at least one animal was protected have been highlighted in bold for easier data interpretation.

${ }^{\text {a }}$ Data indicate: number of mice protected / number of mice tested. The animals were examined at two pretreatment times- -0.5 and $2 \mathrm{~h}$.

Table S3. Anticonvulsant activity in mice i.p. (dose of $100 \mathrm{mg} / \mathrm{kg}$ ) - compounds 48, 49, 52, and $\mathbf{5 3}$

\begin{tabular}{|c|c|c|c|c|c|c|c|c|}
\hline \multirow{3}{*}{ Cmpd } & \multirow{3}{*}{$X$} & \multirow{3}{*}{$\mathrm{m}$} & \multicolumn{2}{|c|}{ MES test } & \multicolumn{2}{|c|}{$6 \mathrm{~Hz}(32 \mathrm{~mA})$ test } & \multicolumn{2}{|c|}{ PTZ test } \\
\hline & & & \multicolumn{2}{|c|}{ Pretreatment times $(\mathrm{h})^{\mathrm{a}}$} & \multicolumn{2}{|c|}{ Pretreatment time $(\mathrm{h})^{\mathrm{a}}$} & \multicolumn{2}{|c|}{ Pretreatment times $(\mathrm{h})^{\mathrm{a}}$} \\
\hline & & & 0.5 & 2.0 & 0.5 & 2.0 & 0.5 & 2.0 \\
\hline 48 & $\mathrm{C}$ & 1 & $3 / 4$ & $1 / 4$ & $3 / 4$ & $1 / 4$ & $1 / 4$ & $0 / 4$ \\
\hline 49 & $\mathrm{~N}$ & 2 & $0 / 4$ & $0 / 4$ & - & - & - & - \\
\hline 52 & - & - & $2 / 4$ & $0 / 4$ & - & - & - & - \\
\hline 53 & - & - & $1 / 4$ & $0 / 4$ & - & - & - & - \\
\hline
\end{tabular}

Ratios where at least one animal was protected have been highlighted in bold for easier data interpretation.

${ }^{a}$ Data indicate: number of mice protected / number of mice tested. The animals were examined at two pretreatment times- -0.5 and $2 \mathrm{~h}$. A dash indicates-not tested. 
Table S4. The detailed data form $s c$ PTZ seizure test for compounds 11, 13, 19, and 22

\begin{tabular}{|c|c|c|c|c|c|}
\hline Compd & $\begin{array}{l}\text { Dose } \\
(\mathrm{mg} / \mathrm{kg})\end{array}$ & $\begin{array}{c}\text { Number of seizure } \\
\text { episodes }\end{array}$ & $\begin{array}{l}\text { Number of } \\
\text { deaths/total }\end{array}$ & $\begin{array}{c}\text { Mortality } \\
(\%)\end{array}$ & $\begin{array}{l}\text { Protection } \\
(\%)\end{array}$ \\
\hline Vehicle & - & 20 & $5 / 10$ & 50 & - \\
\hline \multirow{3}{*}{11} & 60 & 11 & $0 / 6$ & 0 & 100 \\
\hline & 80 & 5 & $2 / 6$ & 33.3 & 66.7 \\
\hline & 120 & $2^{*}$ & $2 / 6$ & 33.3 & 66.7 \\
\hline \multirow{3}{*}{13} & 40 & 6 & $1 / 6$ & 16.7 & 83.3 \\
\hline & 60 & 3 & $0 / 6$ & 0 & 100 \\
\hline & 80 & $1^{*}$ & $0 / 6$ & 0 & 100 \\
\hline \multirow{3}{*}{19} & 40 & 6 & $2 / 6$ & 33.3 & 66.7 \\
\hline & 60 & 3 & $2 / 6$ & 33.3 & 66.7 \\
\hline & 80 & 6 & $1 / 6$ & 16.7 & 83.3 \\
\hline \multirow{3}{*}{22} & 30 & 6 & $1 / 6$ & 16.7 & 83.3 \\
\hline & 60 & 8 & $1 / 6$ & 16.7 & 83.3 \\
\hline & 80 & 4 & $0 / 6$ & 0 & 100 \\
\hline \multirow[t]{2}{*}{ vehicle } & - & 14 & $3 / 6$ & 50.0 & - \\
\hline & 120 & 5 & $0 / 6$ & 0 & 100 \\
\hline \multirow[t]{3}{*}{ ETX } & 150 & 3 & $0 / 6$ & 0 & 100 \\
\hline & 180 & $1^{*}$ & $0 / 6$ & 0 & 100 \\
\hline & 200 & 4 & $1 / 6$ & 16.7 & 83.3 \\
\hline \multirow[t]{2}{*}{ VPA } & 250 & $2^{*}$ & 0 & 0 & 100 \\
\hline & 300 & $1^{*}$ & 0 & 0 & 100 \\
\hline
\end{tabular}

The tested compounds and valproic acid (VPA) were administered i.p. 30 min and ethosuximide (ETX) $15 \mathrm{~min}$ before the $s c$ PTZ test. ETX and VPA were used as positive control (reference drugs). ${ }^{*} \mathrm{p}<0.05$ $v s$ control group (Fisher's exact test). 
Table S5. Binding/functional profile for anticonvulsant active $(\mathbf{2 7}, \mathbf{4 4})$ vs. inactive (26) compounds

\begin{tabular}{|c|c|c|c|c|}
\hline Binding studies & Source & \multicolumn{3}{|c|}{$\begin{array}{l}\text { \% Inhibition of control specific binding }{ }^{a} \\
\qquad \text { (concentration } \mu \mathrm{M})\end{array}$} \\
\hline Compd & & 26 & 27 & 44 \\
\hline $\mathrm{Na}^{+}$channel (site 2 ) & $\begin{array}{l}\text { rat cerebral } \\
\text { cortex }\end{array}$ & $\begin{array}{c}24.8(100) \\
-3.7(10)\end{array}$ & $\begin{array}{c}85.8(100) \\
25.1(10)\end{array}$ & $\begin{array}{c}45.8(100) \\
2.2(10)\end{array}$ \\
\hline Functional studies & & \multicolumn{3}{|c|}{$\begin{array}{l}\% \text { Inhibition of control agonist response }{ }^{a} \\
\qquad \text { (concentration } \mu \mathrm{M})\end{array}$} \\
\hline $\begin{array}{l}\text { TRPV1 (VR1) }(h) \\
\text { (antagonist effect) }\end{array}$ & $\begin{array}{l}\text { human } \\
\text { recombinant } \\
\text { (CHO cells) }\end{array}$ & $2.5(100)$ & $75.3(100)$ & $68.3(100)$ \\
\hline $\begin{array}{l}\mathrm{Cav}_{1.2} \text { (L-type) }(h) \text { calcium } \\
\text { ion channel cell based } \\
\text { antagonist calcium flux assay }\end{array}$ & $\begin{array}{l}\text { human } \\
\text { recombinant } \\
\text { (HEK-293 cells) }\end{array}$ & $38.0(10)$ & $107.0(10)$ & $97.0(10)$ \\
\hline
\end{tabular}

${ }^{a}$ Results showing activity higher than $\mathbf{5 0 \%}$ are considered to represent significant effects of the test compounds; results showing an inhibition between $25 \%$ and $50 \%$ are indicative of weak effect; results showing an inhibition lower than $25 \%$ are not considered significant and mostly attributable to variability of the signal around the control level. Binding or functional studies were performed commercially in Cerep Laboratories (Poitiers, France). 

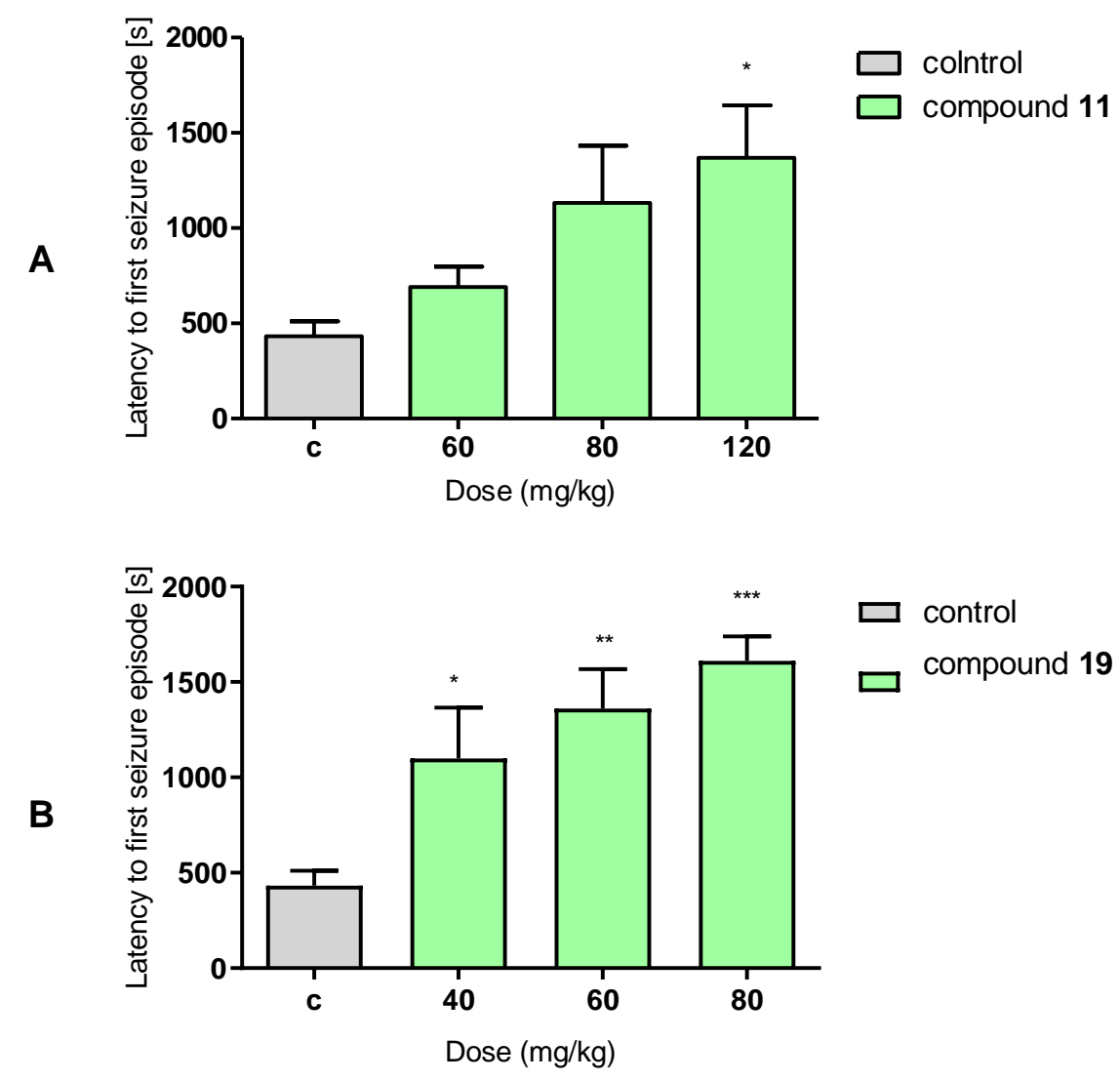

Figure S1 A and B. Anticonvulsant activity of compounds $\mathbf{1 1}$ and $\mathbf{1 9}$ in the $s c$ PTZ test. Each value represents the mean \pm SEM obtained from 6-10 mice. Statistical analysis: one-way analysis of variance (ANOVA), followed by Dunnett's post hoc test. The compounds were administered i.p. $0.5 \mathrm{~h}$ before the test. Significant difference compared to the control group: *p $<0.05, * * \mathrm{p}<0.01, * * * \mathrm{p}<0.001$ 


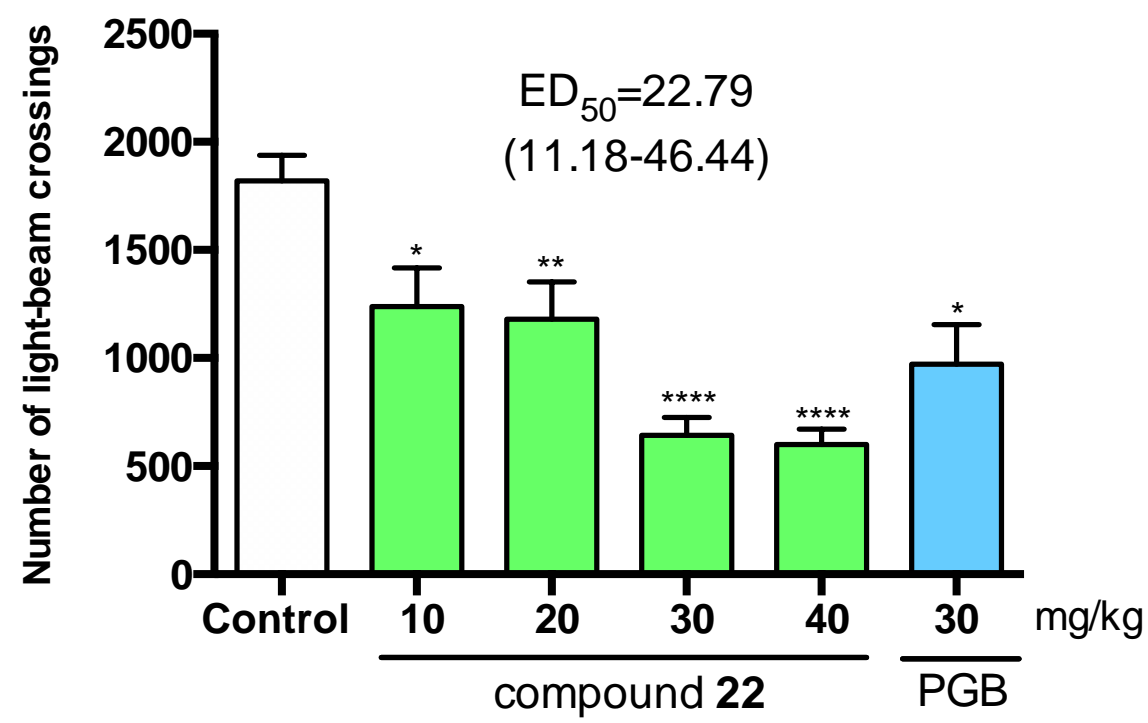

Figure S2. The influence of compound 22 and PGB on spontaneous activity of mice. Results are shown as number of light beam crossings in $30 \mathrm{~min}$ period after i.p. injection of the test compound. Each value represents the mean \pm S.E.M. for 8-10 animals. Statistical analysis: oneway ANOVA followed by post hoc Dunnett's test. Statistical significance compared to vehicletreated animals (Tween): ${ }^{*} \mathrm{p}<0.05, * * \mathrm{p}<0,01, * * * * \mathrm{p}<0.0001$.

The mean number of light-beam crossings in the vehicle-treated animals was $1.82 \pm 0.12 \times 10^{3}$ measured during the whole 30-min-long period of observation. The values were significantly decreased in animals treated with test compound at doses $10,20,30$ and $40 \mathrm{mg} / \mathrm{kg}$ by $31.94 \%$ $(\mathrm{p}<0.05), 35.18 \%(\mathrm{p}<0.01), 64.64 \%(\mathrm{p}<0.0001)$ and $66.93 \%(\mathrm{p}<0.0001)$, respectively. The $\mathrm{ED}_{50}$ value obtained in this test was $22.7 \mathrm{mg} / \mathrm{kg}$, it was approximately 1.8 -fold higher than the $\mathrm{ED}_{50}$ value obtained in the late phase of the formalin test. The administration of PGB at the dose of $30 \mathrm{mg} / \mathrm{kg}$ also resulted in a significant decrease in number of light beam crossings by $45.78 \%(\mathrm{p}<0.05)$. 


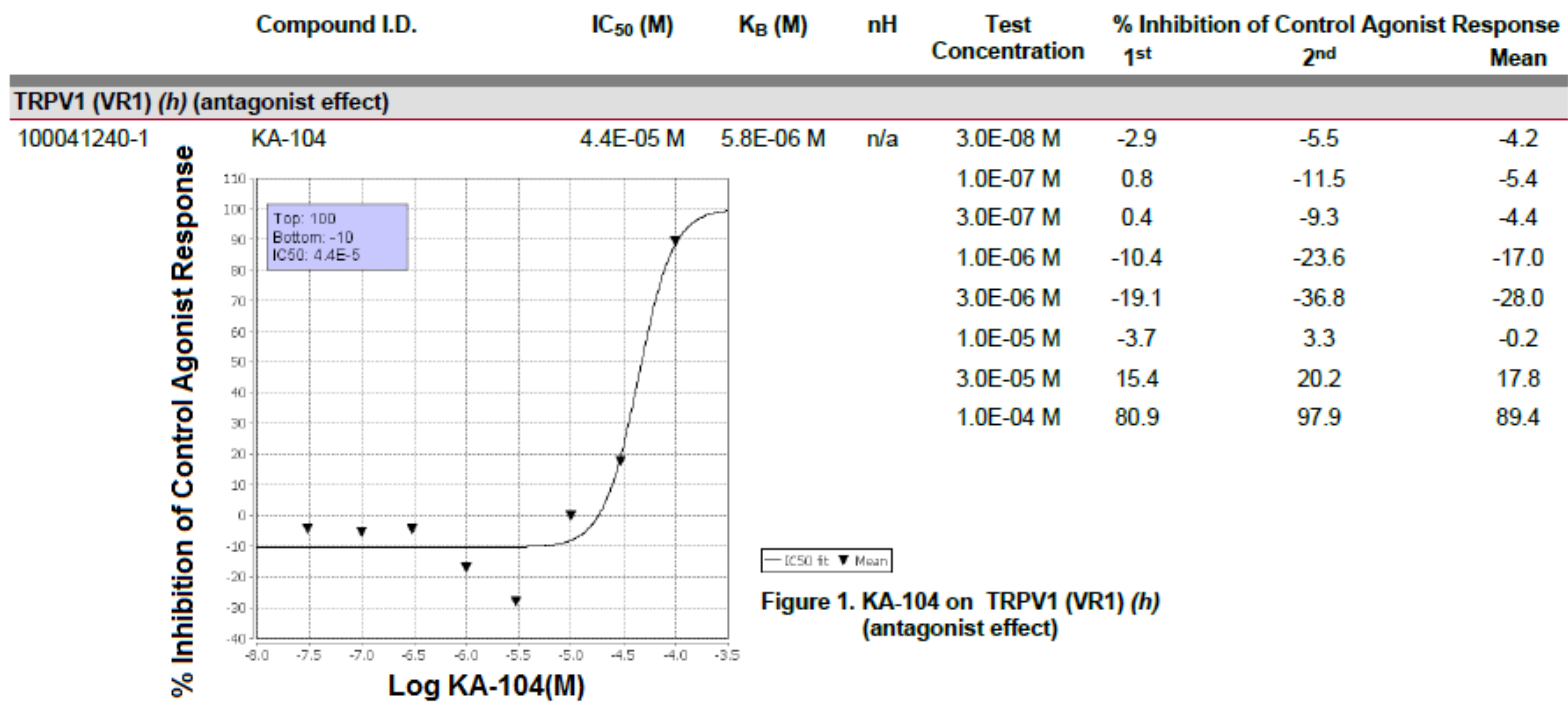

Figure S3. TRPV1 antagonism - $\mathrm{IC}_{50}$ determination and $\mathrm{K}_{\mathrm{B}}$ value for compound 22 (studies were performed in Cerep Laboratories (Poitiers, France).

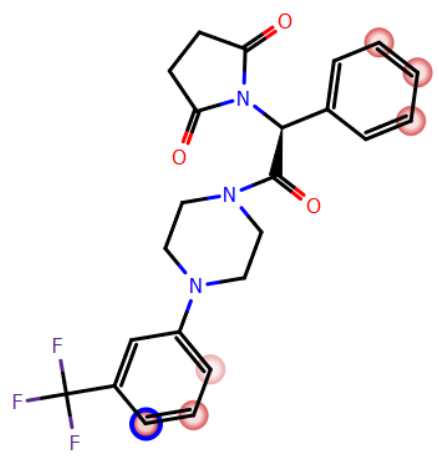

Figure S4. The in silico prediction of the most probably sites of metabolism of 22 by using MetaSite 5.1.1 software. The darker red color of the marked functional group indicates its higher probability to be involved in the metabolism pathway. The blue circle marked the site involved in metabolism with the highest probability $(100 \%)$. 


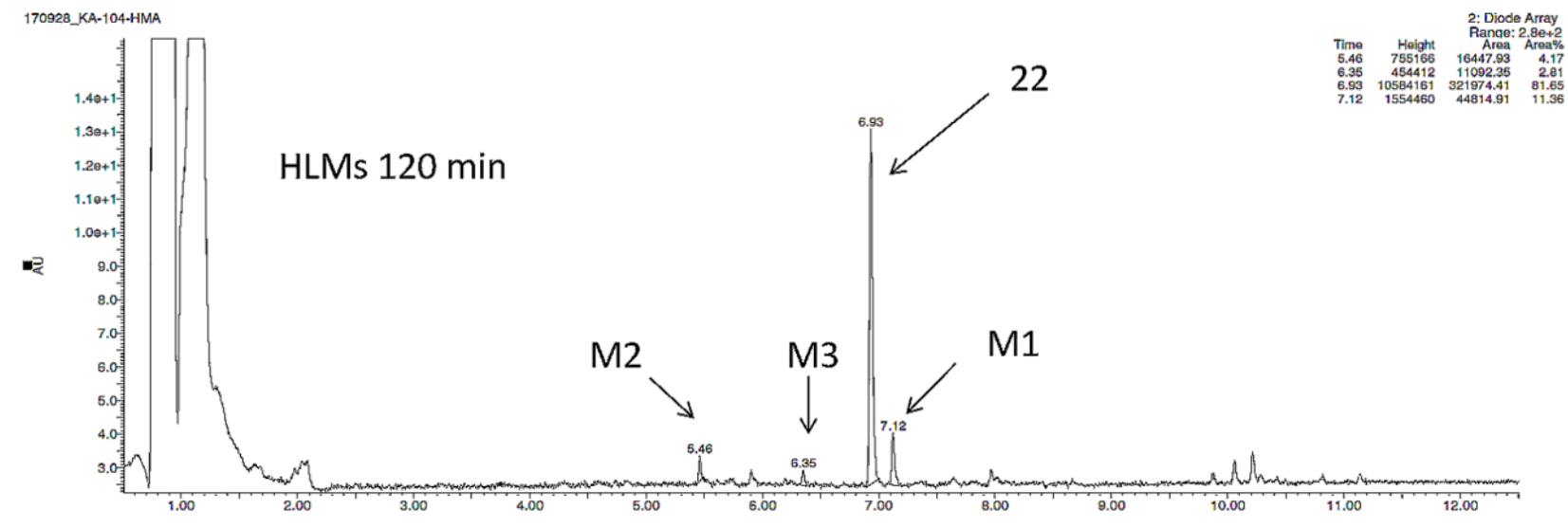

Figure S5. The UPLC spectrum after 120 min reaction of 22 with HLMs. Three metabolites (M1-M3) were observed. Around 20\% of 22 was metabolized with HLMs.

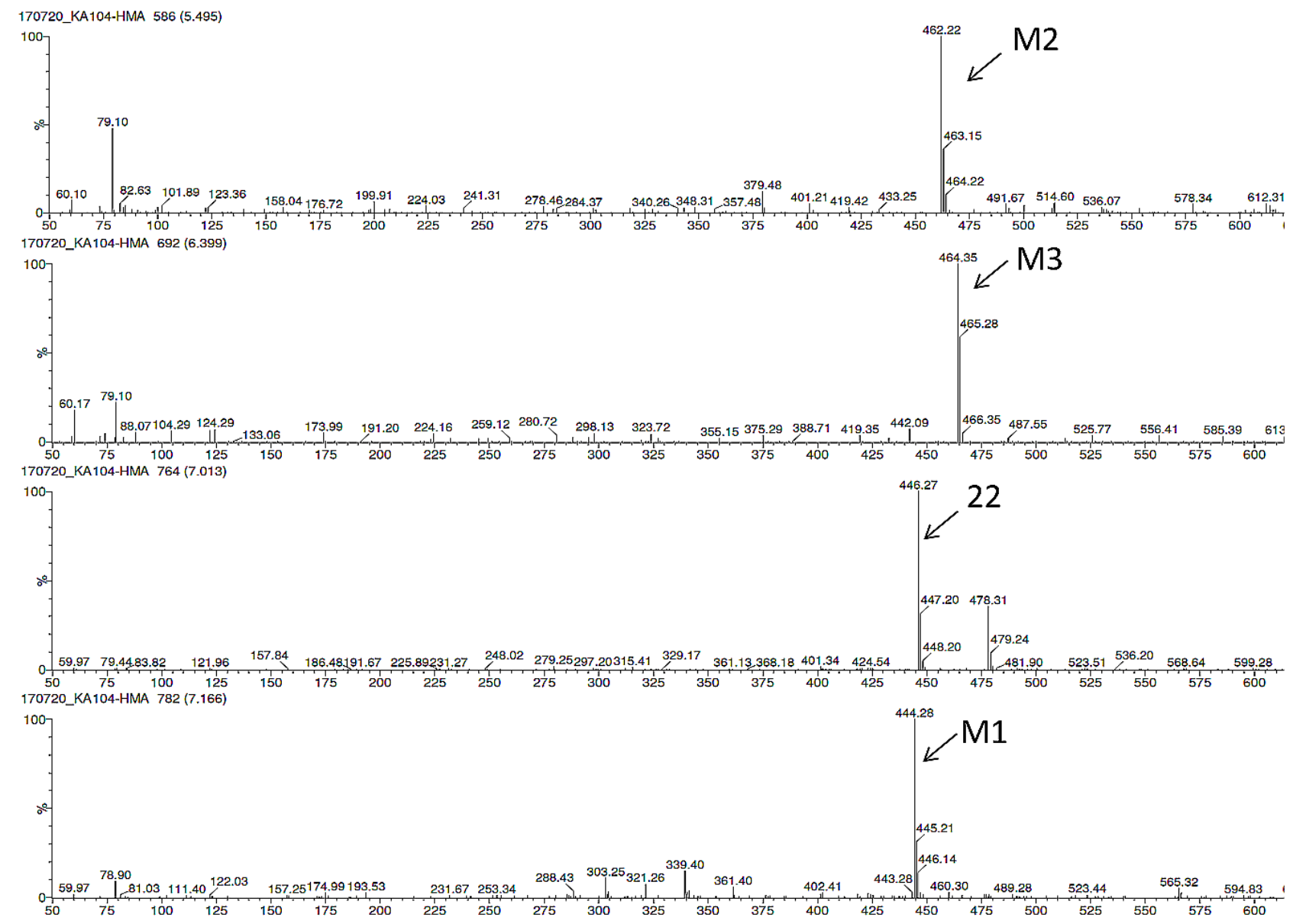

Figure S6. MS spectra of compound 22 and its metabolites M1-M3 obtained after incubation with HLMs. 


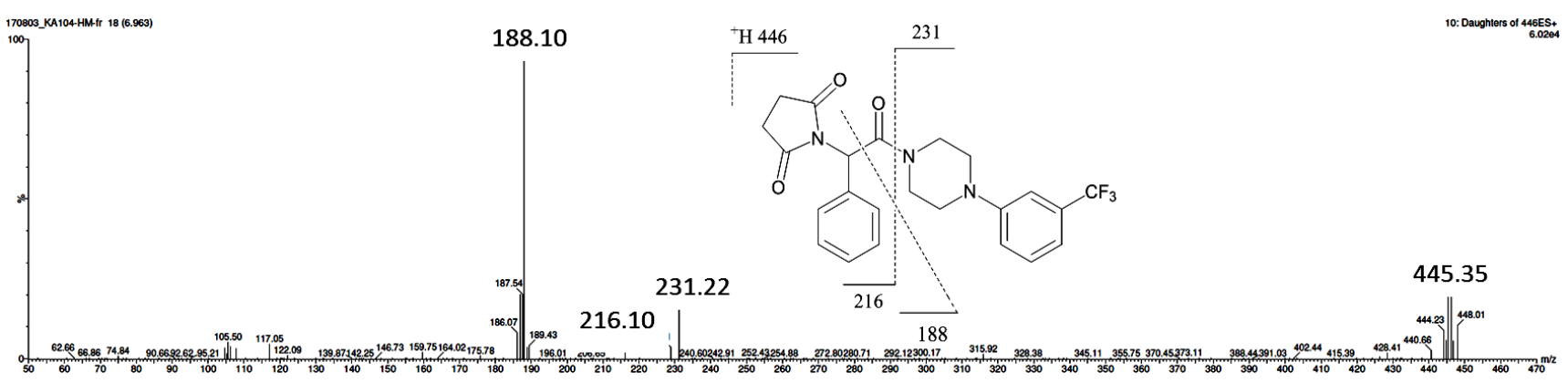

Figure S7. The MS fragmentation analysis of 22.

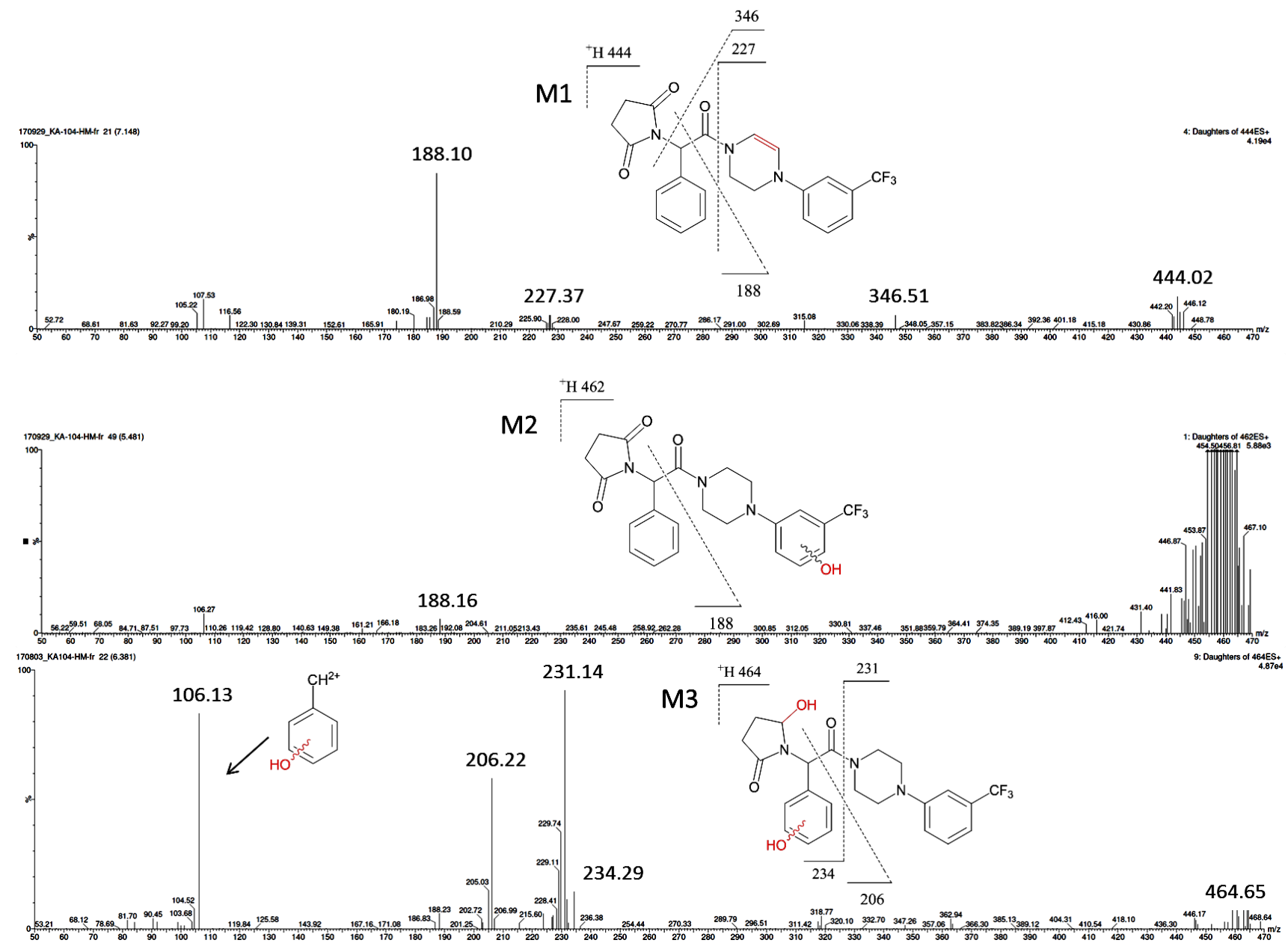

Figure S8. The most probably structures of metabolites M1-M3 based on the MetaSite metabolites' prediction and MS fragmentation analysis. 


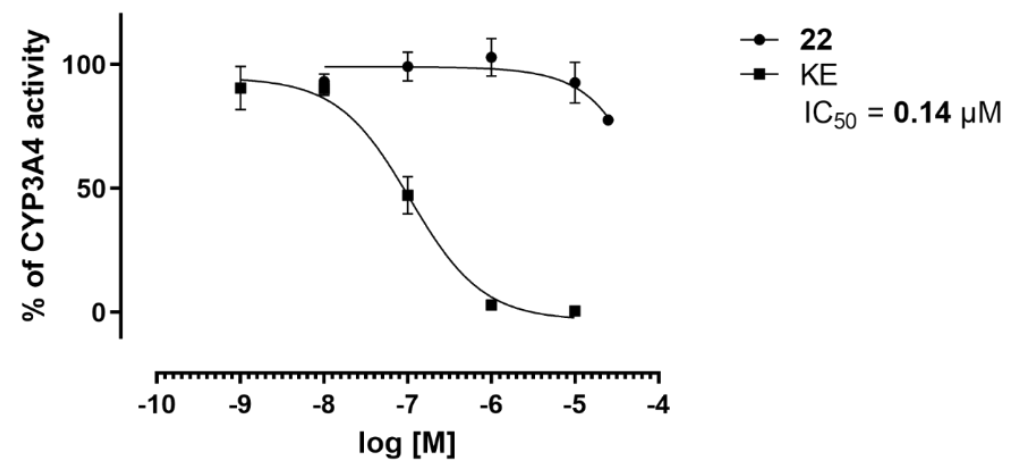

Figure S9. The influence of 22 and the reference inhibitor ketoconazole (KE) on CYP3A4 activity.

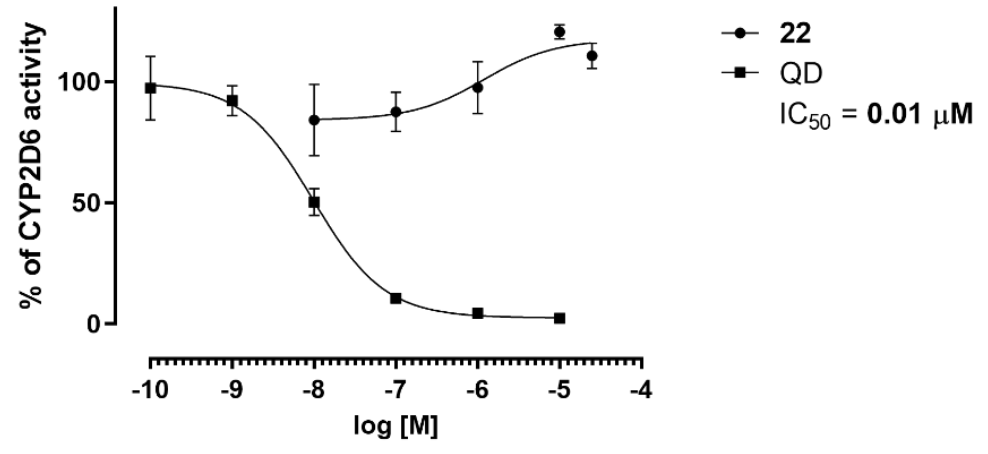

Figure S10. The influence of $\mathbf{2 2}$ and the reference inhibitor quinidine (QD) on CYP2D6 activity. 


\section{In vitro ADME-Tox studies - materials and methods}

\section{Chemicals}

The reference compounds used during in vitro study, as follows: doxorubicin (DX), caffeine (CFN), carbonyl cyanide 3-chlorophenylhydrazone (CCCP), ketoconazole (KE), norfloxacin (NFX), quinidine (QD) were provided by Sigma-Aldrich (St. Louis, MO, USA).

\section{Metabolic stability}

Human liver microsomes (HLMs) were purchased form Sigma-Aldrich (St. Louis, MO, USA). The NADPH Regeneration System was purchased from Promega (Madison, WI, USA). All experiments were performed as described before. ${ }^{1,2}$ For determination of the half-life $\left(\mathrm{t}_{1 / 2}\right)$ and intrinsic clearance $\left(\mathrm{CL}_{\text {int }}\right)$ parameters the four independent reactions with $22(50 \mu \mathrm{M})$ were performed and terminated by the addition of cold methanol containing internal standard (IS) at different points: 5, 15, 30, $45 \mathrm{~min}$. The $\mathrm{t}_{1 / 2}$ and $\mathrm{CL}_{\text {int }}$ values were calculated using Eq. 1, 2, and 3 , according to Obach et al. ${ }^{3}$ For the metabolic pathways determination the reactions were terminated after $120 \mathrm{~min}$.

$t_{1 / 2}=\frac{\ln 2}{-k}$

(Eq. 1)

(Eq. 2)

$C L_{\text {int (mouse) }}=\frac{0.693}{{\text { in vitro } t_{1 / 2}}_{\text {vicrosomes }}} \times \frac{\text { ml incubation }}{\text { mg micros liver }} \times \frac{140 \text { gm liver }}{\mathrm{kg} \mathrm{b.w.}}$

$C L_{\text {int (human })}=\frac{0.693}{{\text { in vitro } t_{1 / 2}}_{\text {ng microsomes }}} \times \frac{\text { ml incubation }}{\text { mg liver }} \times \frac{20 \mathrm{gm} \text { liver }}{\mathrm{kg} \mathrm{b.w.}}$

\section{Influence on CYP3A4 and CYP2D6 activity}

The luminescent CYP3A4 P450-Glo ${ }^{\mathrm{TM}}$ and CYP2D6 P450-Glo TM assays and protocols were provided by Promega (Madison, WI, USA). All experiments were performed as described before. ${ }^{4}$ Each compound was tested in triplicate at the final concentrations similar for both CYP3A4 and CYP2D6 assays in range from 0.025 to $25 \mu \mathrm{M}$. The $\mathrm{IC}_{50}$ values of the reference compounds ketoconazole and quinidine were estimated previously. ${ }^{5}$ The luminescent signal was measured by using a microplate reader EnSpire (PerkinElmer, Waltham, MA USA). 


\section{Radioligand binding/functional assays}

Binding studies were performed commercially in Cerep Laboratories (Poitiers, France) using testing procedures described elsewhere:

\begin{tabular}{|c|c|}
\hline Binding studies & Ref. \\
\hline $\mathrm{Na}^{+}$channel (site 2$)$ & 6 \\
\hline $\begin{array}{l}\text { L-type } \mathrm{Ca}^{2+} \\
\text { (dihydropyridine site, antagonist radioligand) }\end{array}$ & 7 \\
\hline $\begin{array}{l}\text { L-type } \mathrm{Ca}^{2+} \\
\text { (diltiazem site, antagonist radioligand) }\end{array}$ & 8 \\
\hline $\begin{array}{l}\text { L-type } \mathrm{Ca}^{2+} \\
\text { (verapamil site, antagonist radioligand) }\end{array}$ & 9 \\
\hline NMDA (antagonist radioligand) & 10 \\
\hline $\mathrm{Na}^{+}$channel (site 1$)$ & 11 \\
\hline $\begin{array}{l}\mathrm{N} \text {-type } \mathrm{Ca}^{2+} \\
\text { (antagonist radioligand) }\end{array}$ & 12 \\
\hline $\begin{array}{l}\text { GABA transporter } \\
\text { (antagonist radioligand) }\end{array}$ & 13 \\
\hline Potassium channel (hERG) & 14 \\
\hline \multicolumn{2}{|l|}{ Functional studies } \\
\hline TRPV1 (VR1) $(h)$ (antagonist effect) & 15 \\
\hline $\operatorname{Cav}_{1.2}$ (L-type) (h) calcium ion channel cell based antagonist calcium flux assay & 16,17 \\
\hline
\end{tabular}




\section{References}

(1) Latacz, G.; Lubelska, A.; Jastrzębska-Więsek, M.; Partyka, A.; Sobiło, A.; Olejarz, A.; Kucwaj-Brysz, K.; Satała, G.; Bojarski, A. J.; Wesołowska, A.; Kieć-Kononowicz, K.; Handzlik, J. In the Search for a Lead Structure among Series of Potent and Selective Hydantoin 5-HT7R Agents: The Drug-Likeness in Vitro Study. Chem. Biol. Drug Des. 2017, 90 (6), 1295-1306. https://doi.org/10.1111/cbdd.13106.

(2) Latacz, G.; Lubelska, A.; Jastrzębska-Więsek, M.; Partyka, A.; Kucwaj-Brysz, K.; Wesołowska, A.; Kieć-Kononowicz, K.; Handzlik, J. MF-8, a Novel Promising Arylpiperazine-Hydantoin Based 5-HT7 Receptor Antagonist: In Vitro Drug-Likeness Studies and in Vivo Pharmacological Evaluation. Bioorg. Med. Chem. Lett. 2018, 28 (5), 878-883. https://doi.org/10.1016/j.bmcl.2018.02.003.

(3) Obach, R. S. Prediction of Human Clearance of Twenty-Nine Drugs from Hepatic Microsomal Intrinsic Clearance Data: An Examination of In Vitro Half-Life Approach and Nonspecific Binding to Microsomes. Drug Metab. Dispos. 1999, 27 (11), 1350 1359.

(4) Socała, K.; Mogilski, S.; Pieróg, M.; Nieoczym, D.; Abram, M.; Szulczyk, B.; Lubelska, A.; Latacz, G.; Doboszewska, U.; Wlaź, P.; Kamiński, K. KA-11, a Novel Pyrrolidine-2,5-Dione Derived Broad-Spectrum Anticonvulsant: Its Antiepileptogenic, Antinociceptive Properties and in Vitro Characterization. ACS Chem. Neurosci. 2019, 10 (1), 636-648. https://doi.org/10.1021/acschemneuro.8b00476.

(5) Sadek, B.; Saad, A.; Latacz, G.; Kuder, K.; Olejarz, A.; Karcz, T.; Stark, H.; KiećKononowicz, K. Non-Imidazole-Based Histamine H3 Receptor Antagonists with Anticonvulsant Activity in Different Seizure Models in Male Adult Rats. Drug Des. Devel. Ther. 2016, 10, 3879-3898. https://doi.org/10.2147/DDDT.S116192.

(6) Brown, G. B. 3H-Batrachotoxinin-A Benzoate Binding to Voltage-Sensitive Sodium Channels: Inhibition by the Channel Blockers Tetrodotoxin and Saxitoxin. J. Neurosci. 1986, 6 (7), 2064-2070. https://doi.org/10.1523/JNEUROSCI.06-07-02064.1986.

(7) Gould, R. J.; Murphy, K. M.; Snyder, S. H. [3H]Nitrendipine-Labeled Calcium Channels Discriminate Inorganic Calcium Agonists and Antagonists. Proc. Natl. Acad. Sci. 1982, 79 (11), 3656-3660. https://doi.org/10.1073/pnas.79.11.3656.

(8) Schoemaker, H.; Langer, S. Z. [3H]Diltiazem Binding to Calcium Channel Antagonists Recognition Sites in Rat Cerebral Cortex. Eur. J. Pharmacol. 1985, 111 (2), 273-277. https://doi.org/10.1016/0014-2999(85)90768-X.

(9) Reynolds, I. J.; Snowman, A. M.; Snyder, S. H. (-)-[3H] Desmethoxyverapamil Labels Multiple Calcium Channel Modulator Receptors in Brain and Skeletal Muscle Membranes: Differentiation by Temperature and Dihydropyridines. J. Pharmacol. Exp. Ther. 1986, 237 (3), 731-738.

(10) Sills, M. A.; Fagg, G.; Pozza, M.; Angst, C.; Brundish, D. E.; Hurt, S. D.; Wilusz, E. J.; Williams, M. [3H]CGP 39653: A New N-Methyl-D-Aspartate Antagonist Radioligand with Low Nanomolar Affinity in Rat Brain. Eur. J. Pharmacol. 1991, 192 (1), 19-24. https://doi.org/10.1016/0014-2999(91)90063-v.

(11) Doucette, G. J.; Logan, M. M.; Ramsdell, J. S.; Van Dolah, F. M. Development and Preliminary Validation of a Microtiter Plate-Based Receptor Binding Assay for Paralytic Shellfish Poisoning Toxins. Toxicon 1997, 35 (5), 625-636. https://doi.org/10.1016/S0041-0101(96)00189-4.

(12) Wagner, J. A.; Snowman, A. M.; Biswas, A.; Olivera, B. M.; Snyder, S. H. OmegaConotoxin GVIA Binding to a High-Affinity Receptor in Brain: Characterization, Calcium Sensitivity, and Solubilization. J. Neurosci. 1988, 8 (9), 3354-3359. https://doi.org/10.1523/JNEUROSCI.08-09-03354.1988. 
(13) Shank, R. P.; Baldy, W. J.; Mattucci, L. C.; Villani, F. J. Ion and Temperature Effects on the Binding of $\gamma$-Aminobutyrate to Its Receptors and the High-Affinity Transport System. J. Neurochem. 1990, 54 (6), 2007-2015. https://doi.org/10.1111/j.14714159.1990.tb04905.x.

(14) Huang, X.-P.; Mangano, T.; Hufeisen, S.; Setola, V.; Roth, B. L. Identification of Human Ether-à-Go-Go Related Gene Modulators by Three Screening Platforms in an Academic Drug-Discovery Setting. Assay Drug Dev. Technol. 2010, 8 (6), 727-742. https://doi.org/10.1089/adt.2010.0331.

(15) Phelps, P. T.; Anthes, J. C.; Correll, C. C. Cloning and Functional Characterization of Dog Transient Receptor Potential Vanilloid Receptor-1 (TRPV1). Eur. J. Pharmacol. 2005, 513 (1-2), 57-66. https://doi.org/10.1016/j.ejphar.2005.02.045.

(16) Sirenko, O.; Crittenden, C.; Callamaras, N.; Hesley, J.; Chen, Y.-W.; Funes, C.; Rusyn, I.; Anson, B.; Cromwell, E. F. Multiparameter in Vitro Assessment of Compound Effects on Cardiomyocyte Physiology Using IPSC Cells. J. Biomol. Screen. 2013, 18 (1), 39-53. https://doi.org/10.1177/1087057112457590.

(17) Xia, M.; Imredy, J. P.; Koblan, K. S.; Bennett, P.; Connolly, T. M. State-Dependent Inhibition of L-Type Calcium Channels: Cell-Based Assay in High-Throughput Format. Anal. Biochem. 2004, 327 (1), 74-81. https://doi.org/10.1016/j.ab.2004.01.003. 


\section{UPLC/MS traces for target compounds}

1-(2-Oxo-1-phenyl-2-(4-phenylpiperazin-1-yl)ethyl)pyrrolidine-2,5-dione (11)

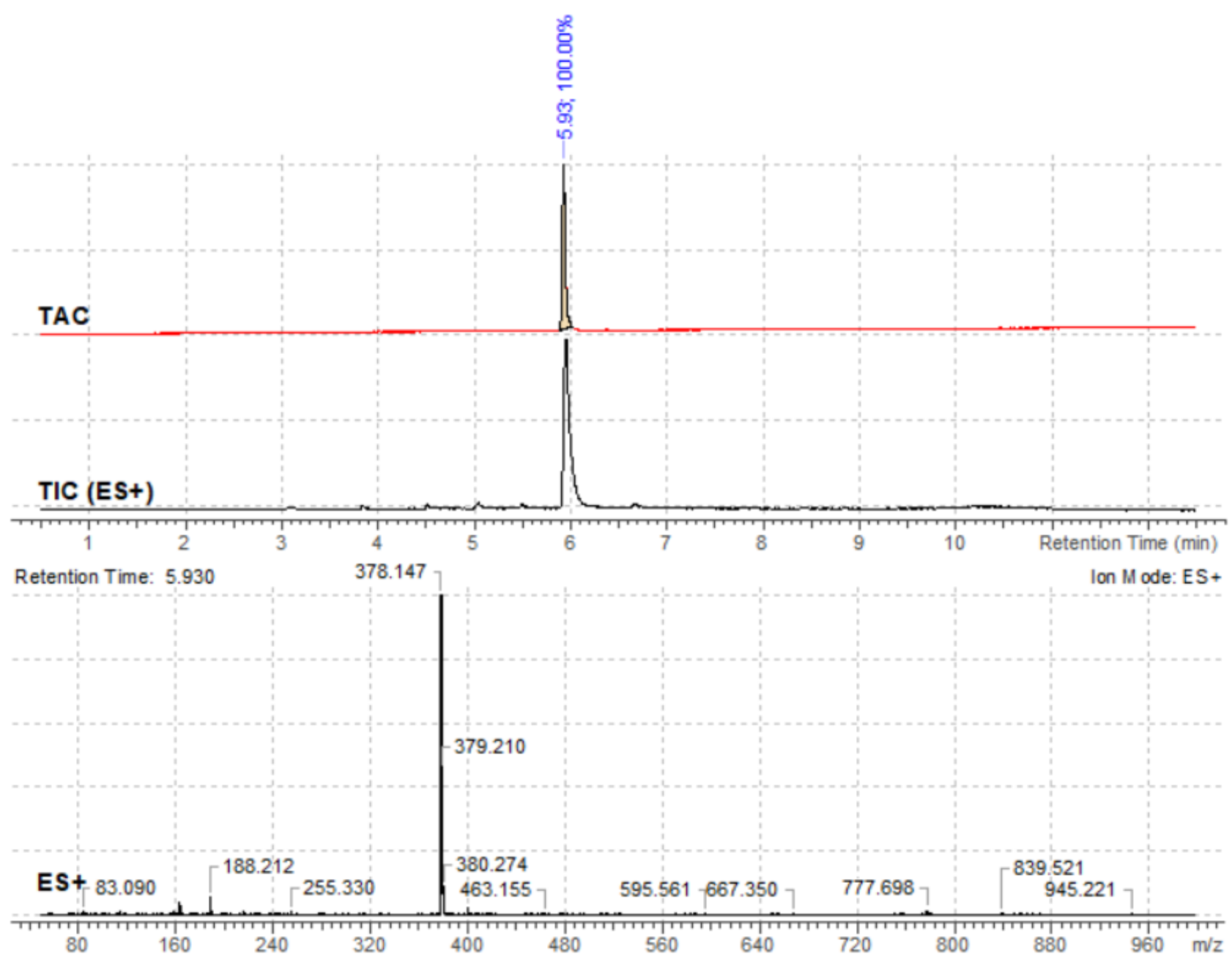


1-(2-(4-(2-Chlorophenyl)piperazin-1-yl)-2-oxo-1-phenylethyl)pyrrolidine-2,5-dione (12).

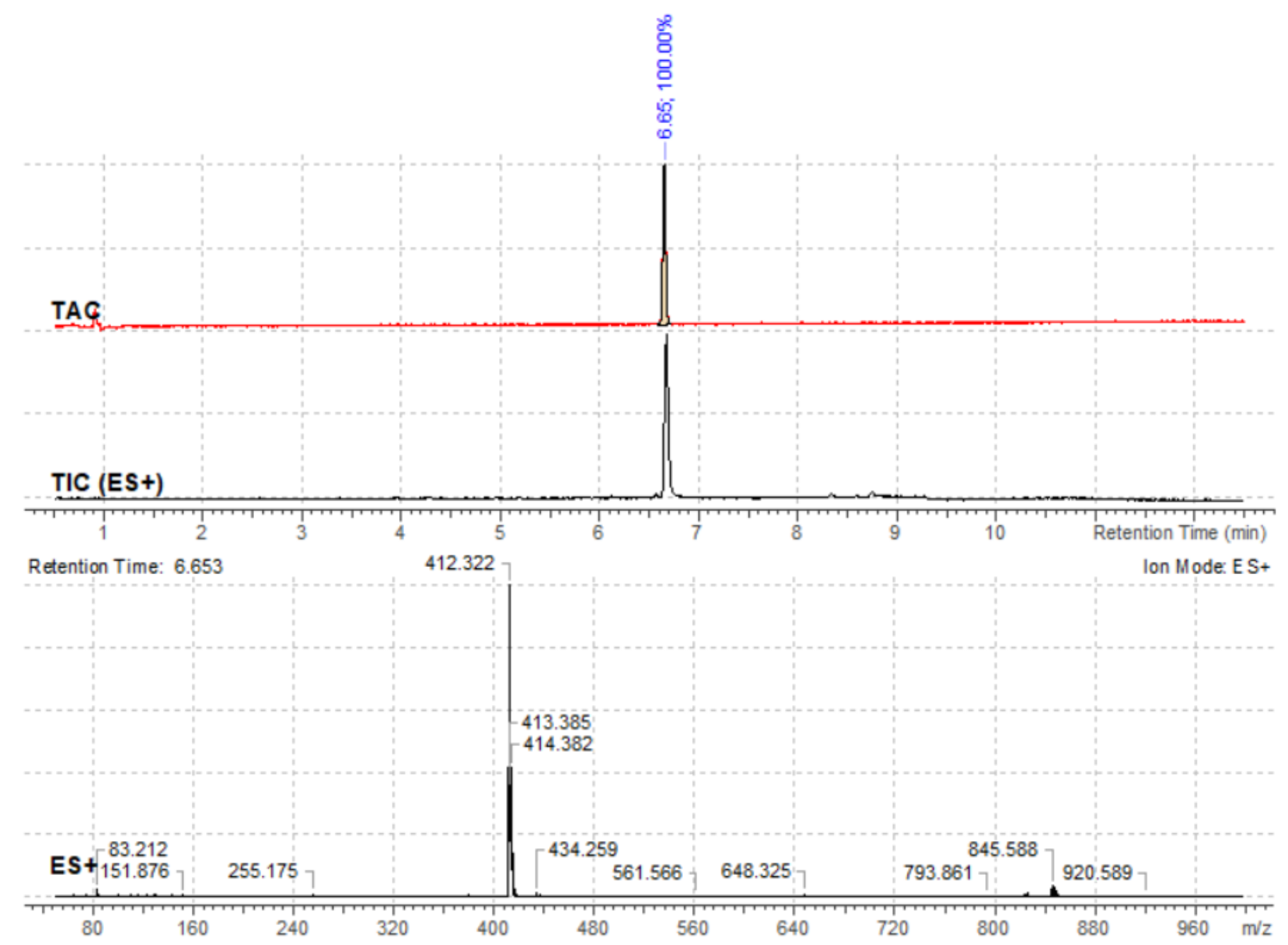


1-(2-(4-(3-Chlorophenyl)piperazin-1-yl)-2-oxo-1-phenylethyl)pyrrolidine-2,5-dione (13)

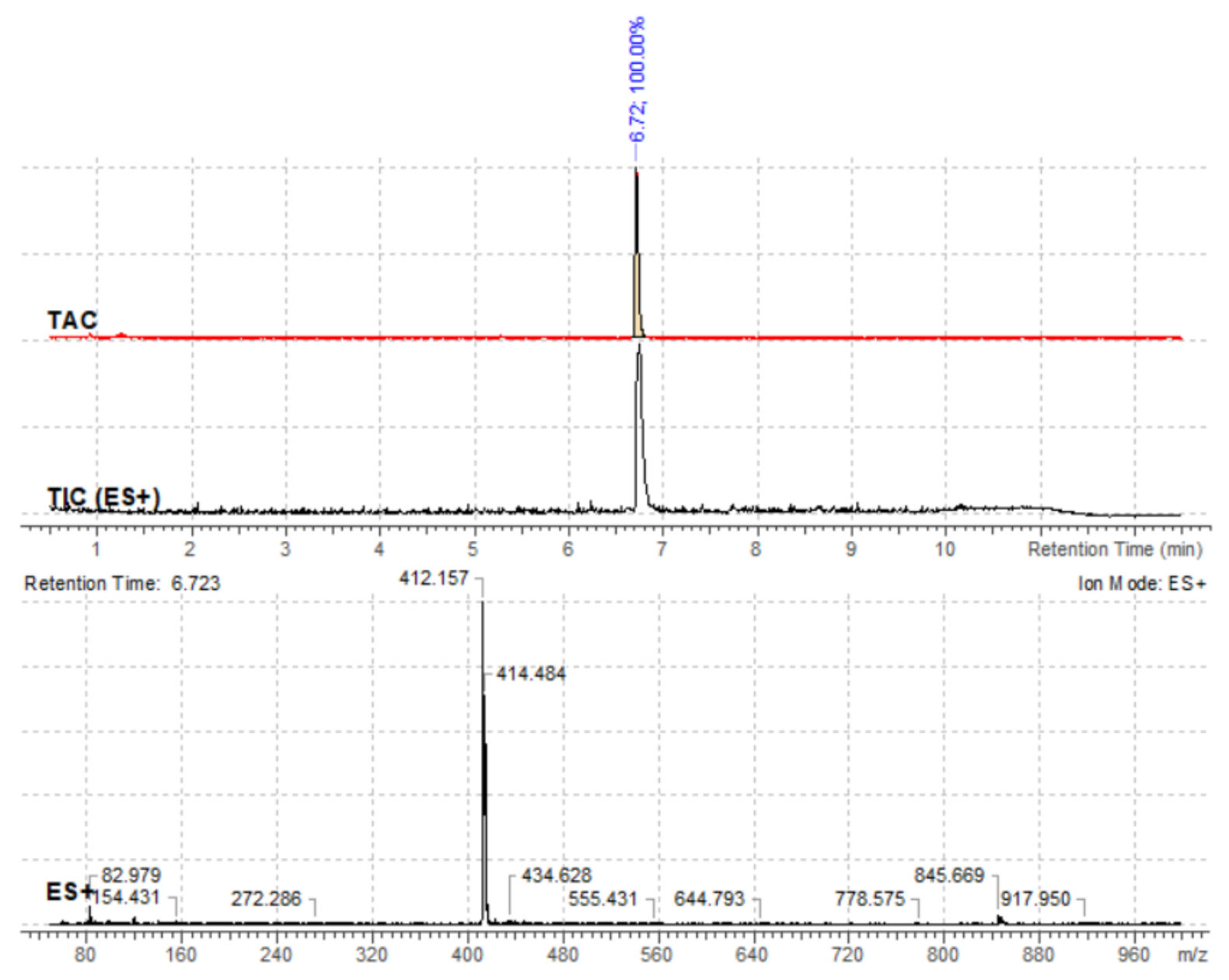


1-(2-(4-(4-Chlorophenyl)piperazin-1-yl)-2-oxo-1-phenylethyl)pyrrolidine-2,5-dione (14).

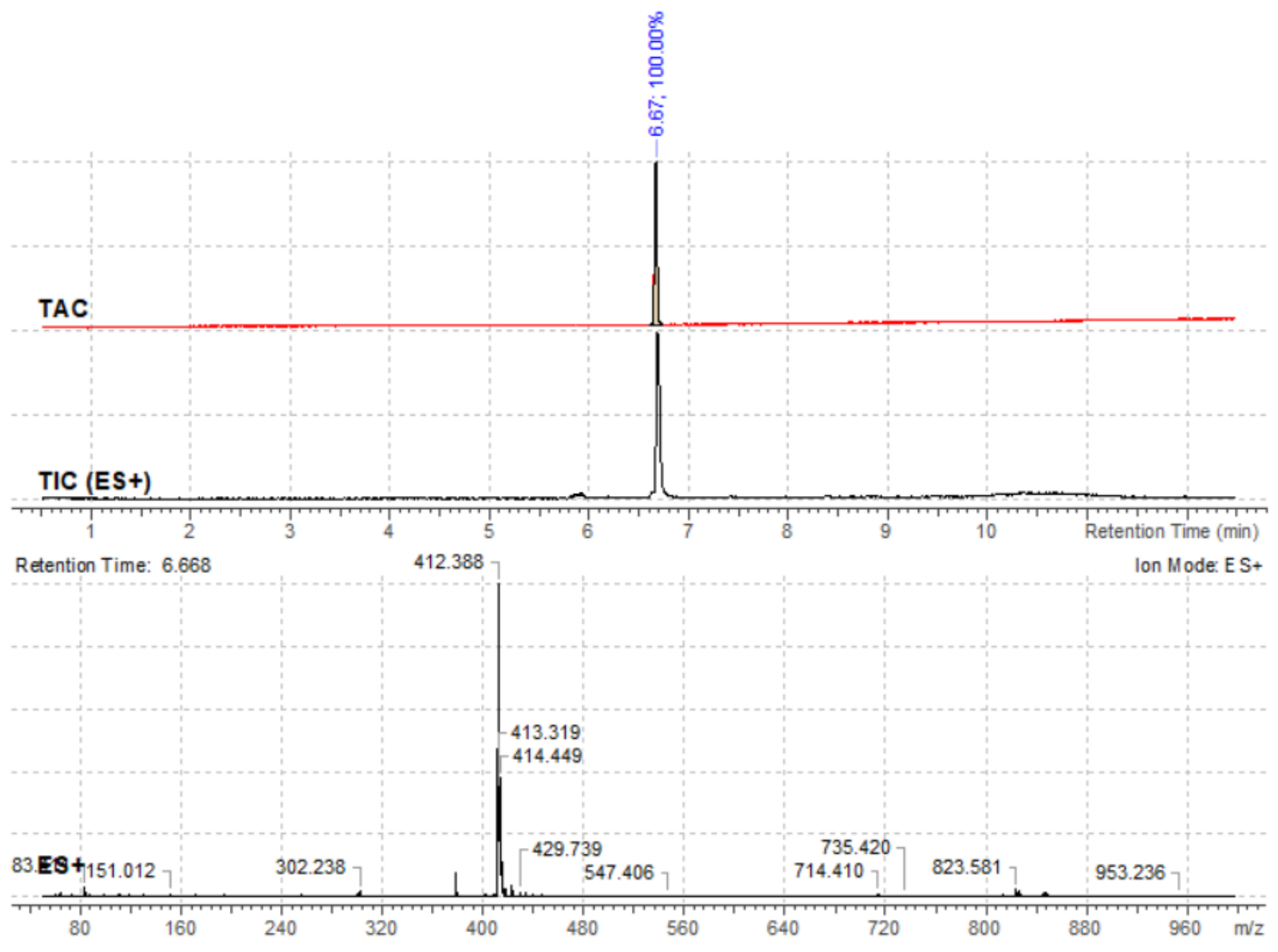


1-(2-(4-(2-Fluorophenyl)piperazin-1-yl)-2-oxo-1-phenylethyl)pyrrolidine-2,5-dione (15).

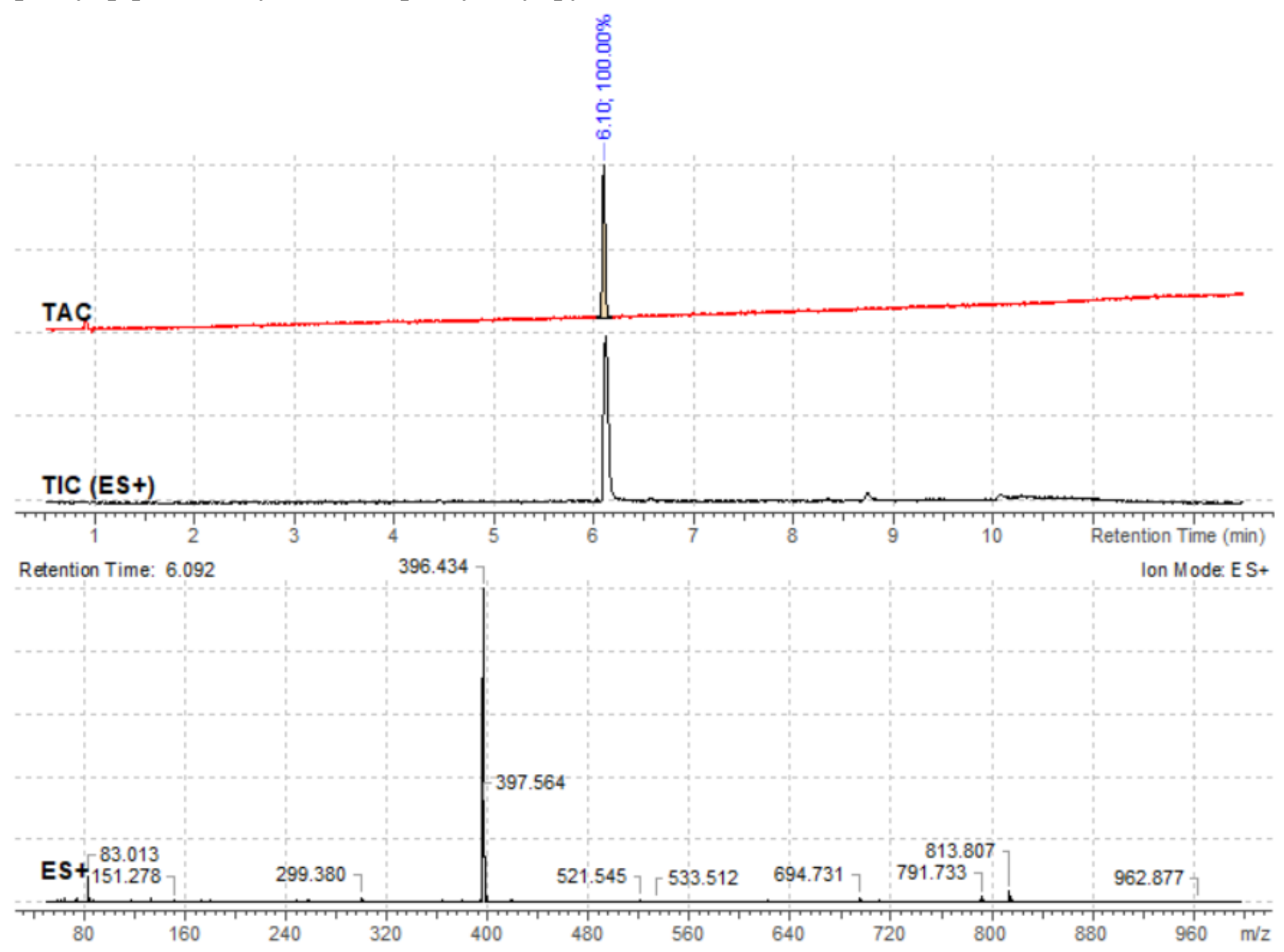


1-(2-(4-(3-Fluorophenyl)piperazin-1-yl)-2-oxo-1-phenylethyl)pyrrolidine-2,5-dione (16).

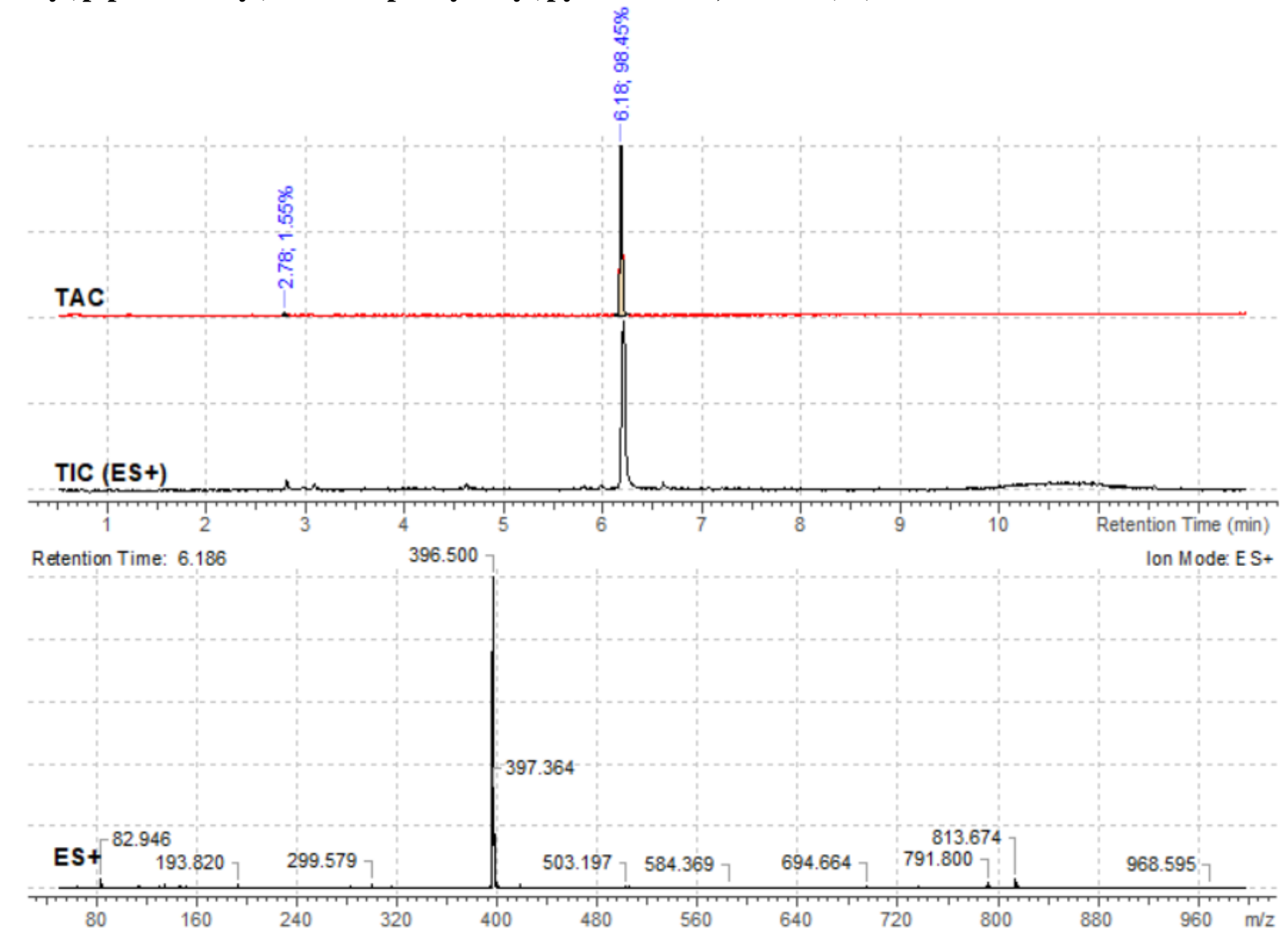


1-(2-(4-(4-Fluorophenyl)piperazin-1-yl)-2-oxo-1-phenylethyl)pyrrolidine-2,5-dione (17).

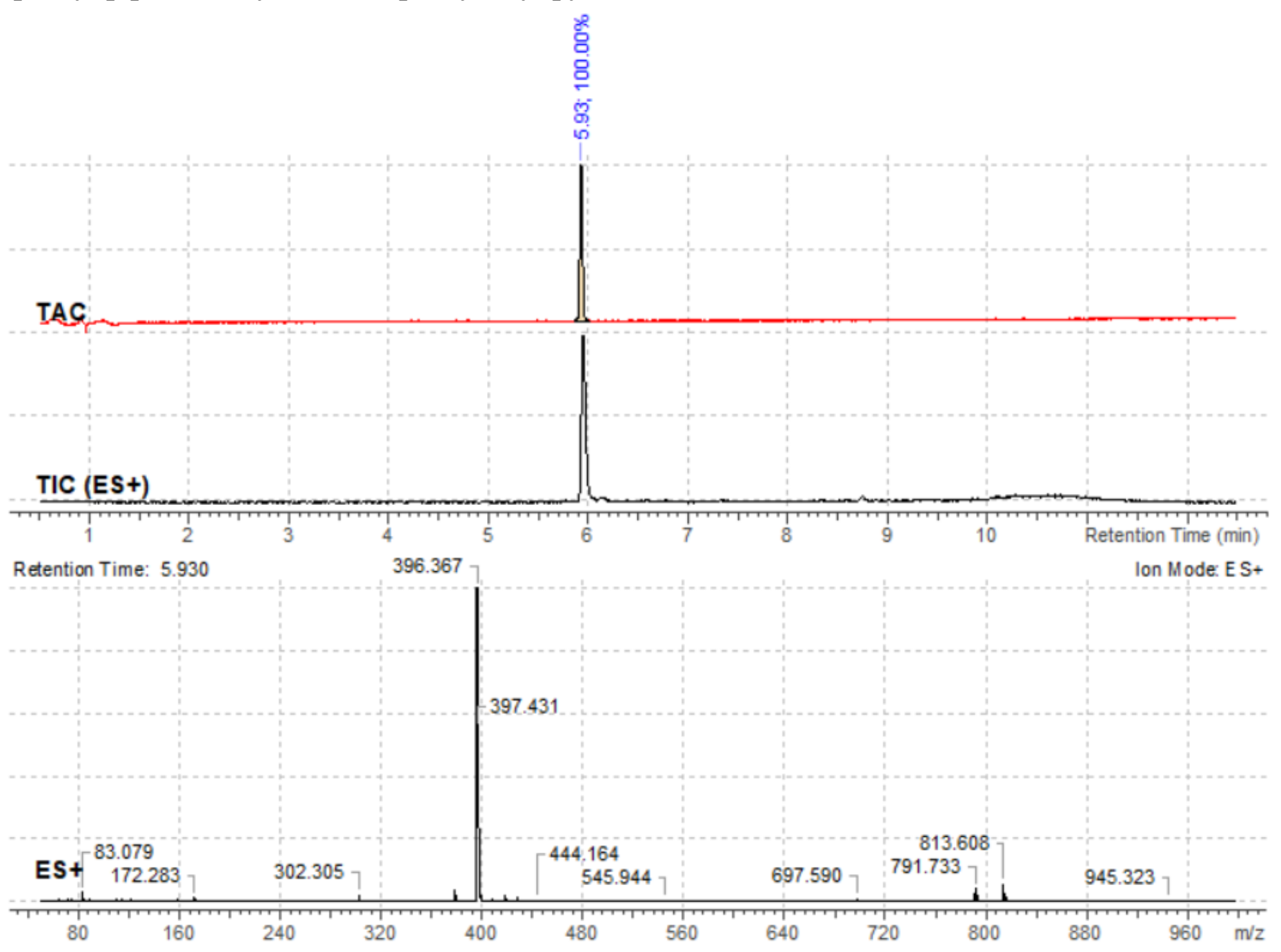


1-(2-Oxo-1-phenyl-2-(4-(o-tolyl)piperazin-1-yl)ethyl)pyrrolidine-2,5-dione (18).

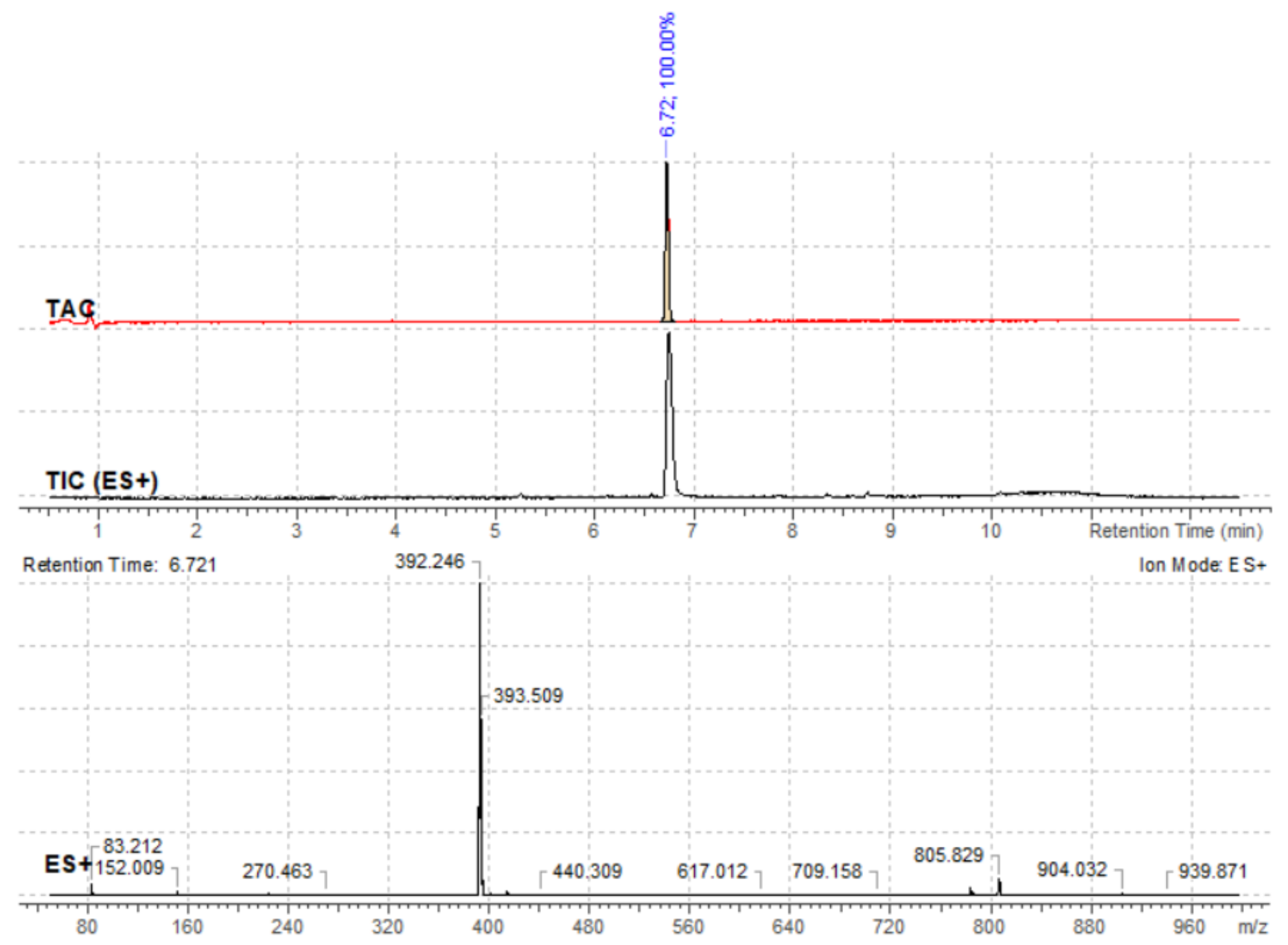


1-(2-Oxo-1-phenyl-2-(4-(m-tolyl)piperazin-1-yl)ethyl)pyrrolidine-2,5-dione (19)

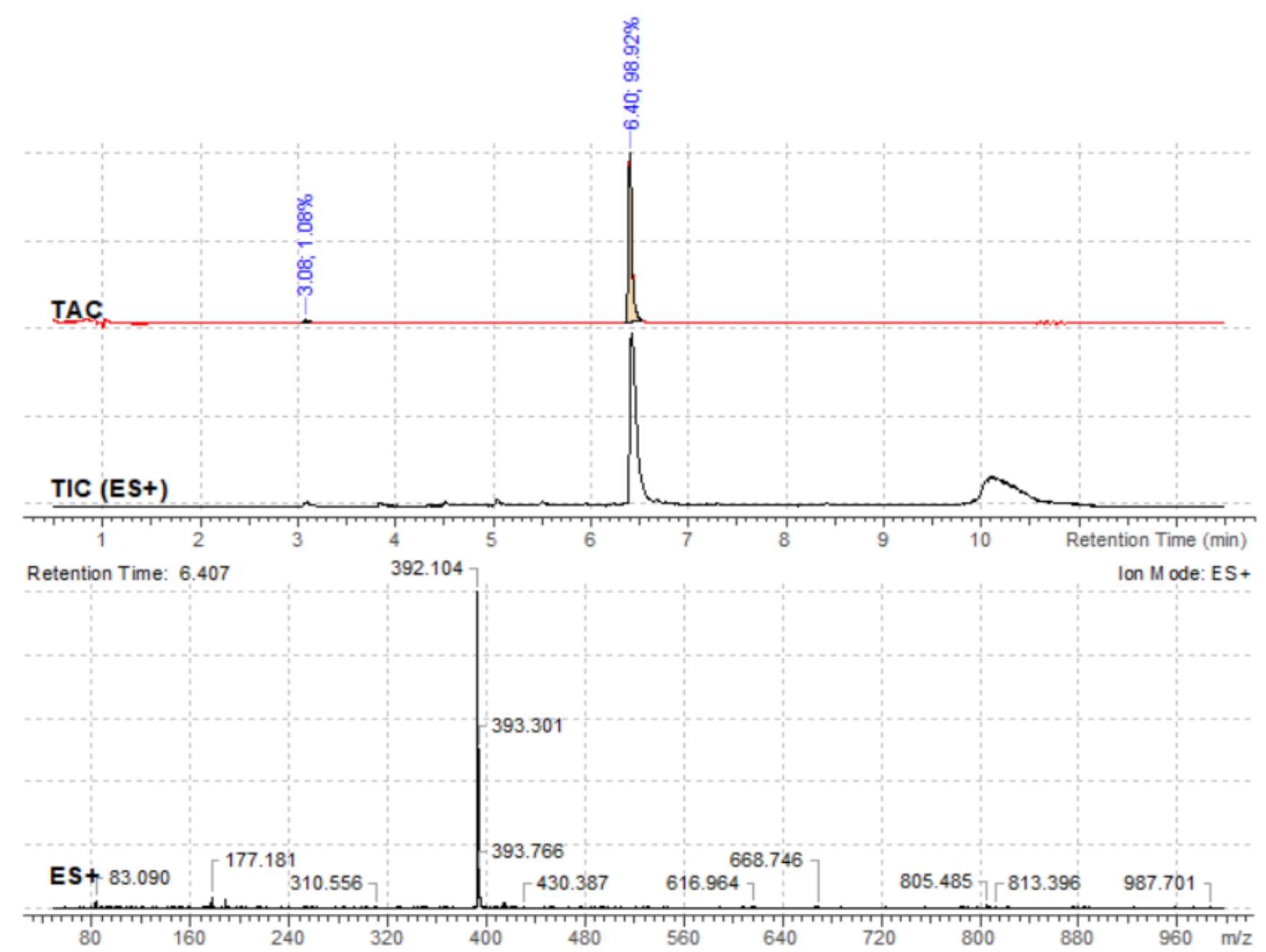


1-(2-Oxo-1-phenyl-2-(4-(p-tolyl)piperazin-1-yl)ethyl)pyrrolidine-2,5-dione (20).

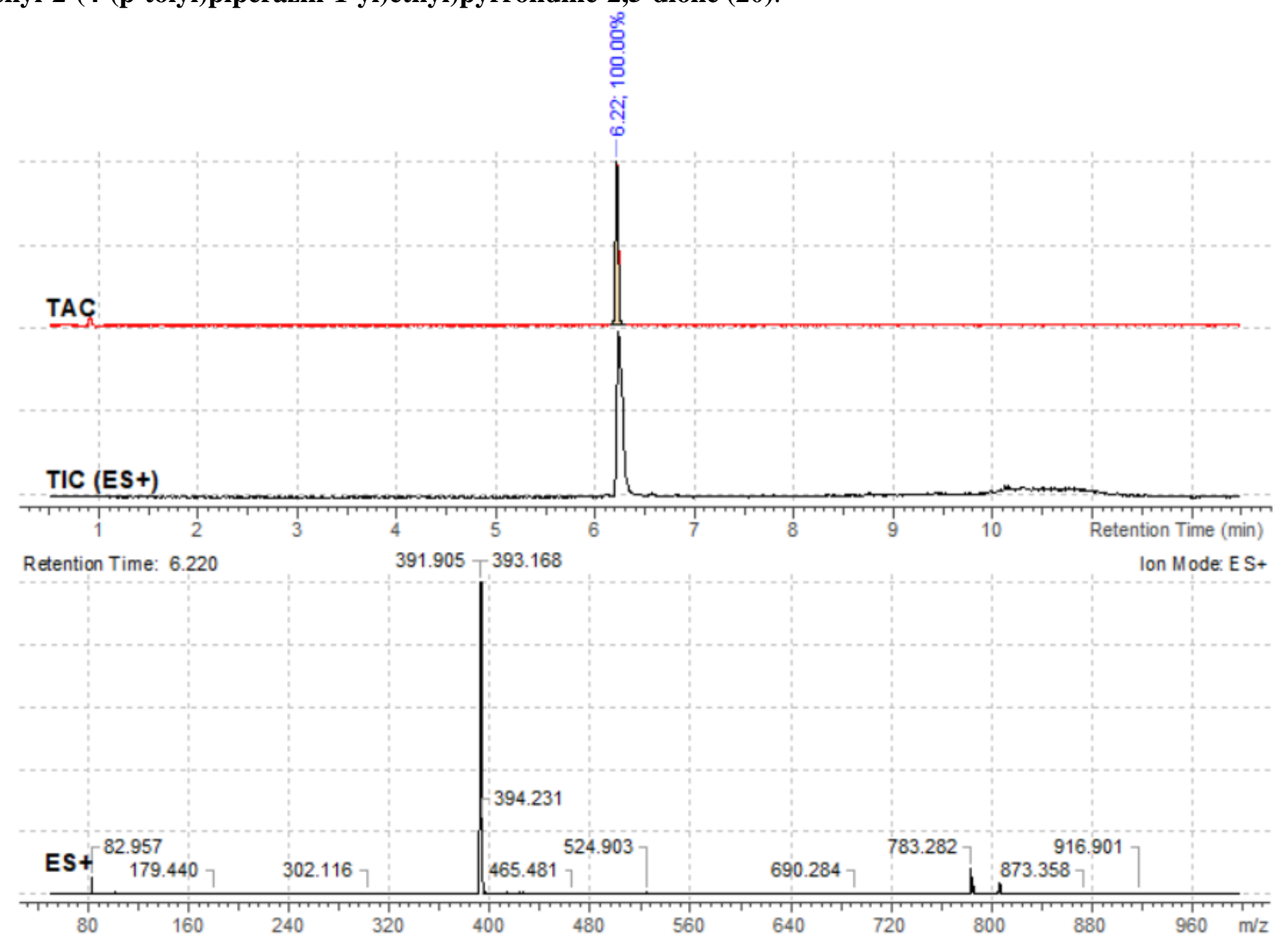


1-(2-Oxo-1-phenyl-2-(4-(2-(trifluoromethyl)phenyl)piperazin-1-yl)ethyl)pyrrolidine-2,5-dione (21).

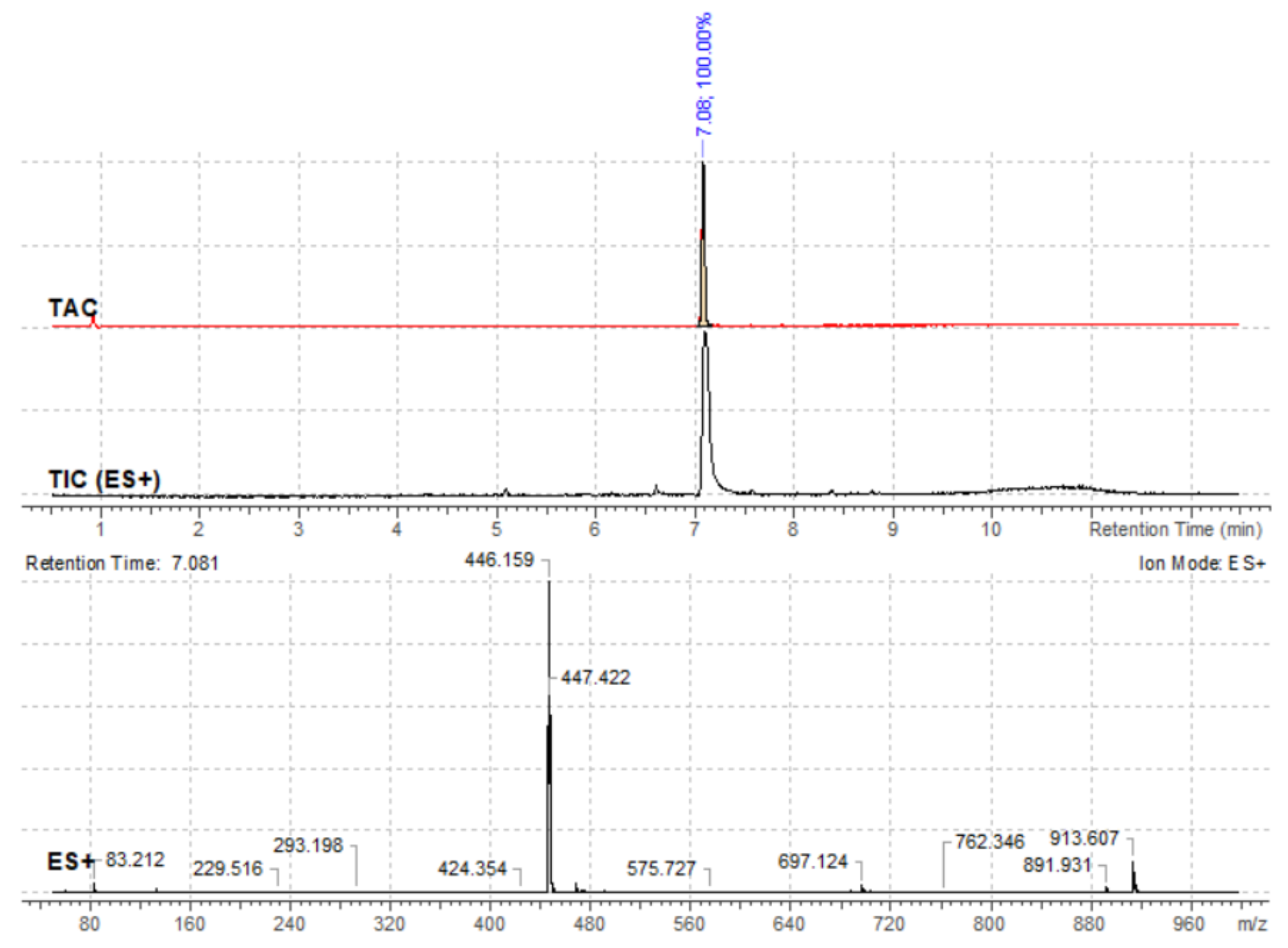


1-(2-Oxo-1-phenyl-2-(4-(3-(trifluoromethyl)phenyl)piperazin-1-yl)ethyl)pyrrolidine-2,5-dione (22)

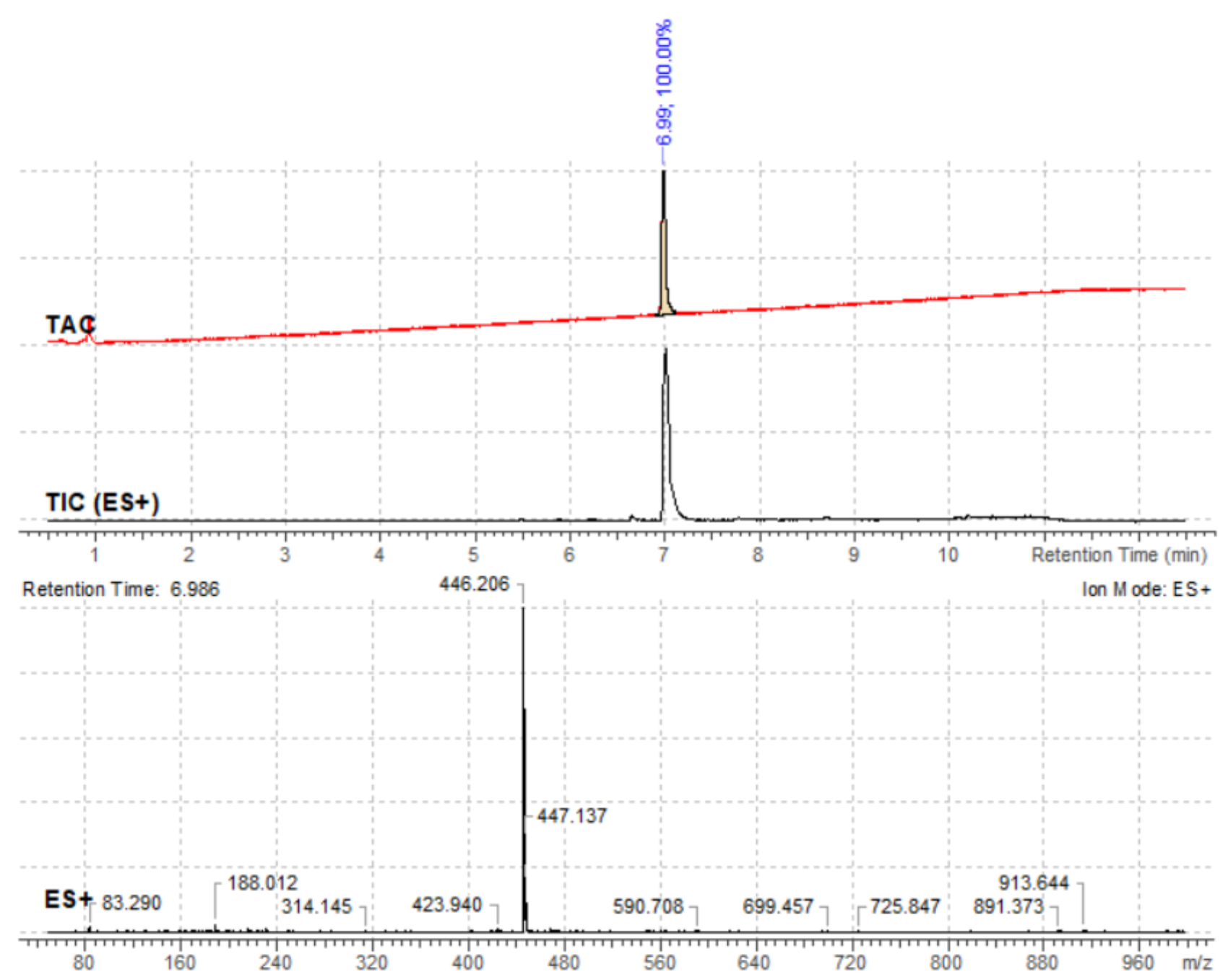


1-(2-Oxo-1-phenyl-2-(4-(4-(trifluoromethyl)phenyl)piperazin-1-yl)ethyl)pyrrolidine-2,5-dione (23)

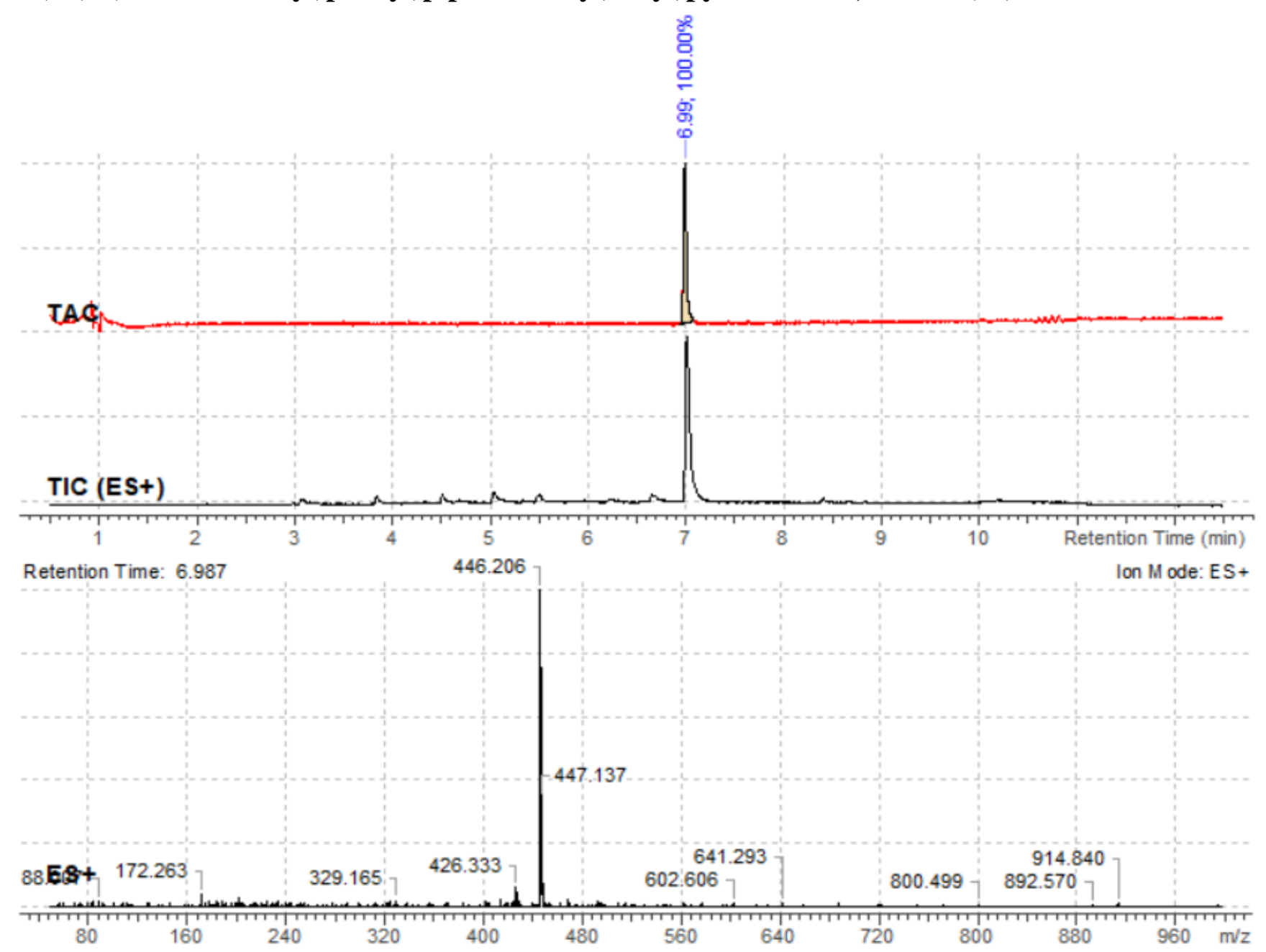


1-(2-(4-(3-Methoxyphenyl)piperazin-1-yl)-2-oxo-1-phenylethyl)pyrrolidine-2,5-dione (24).

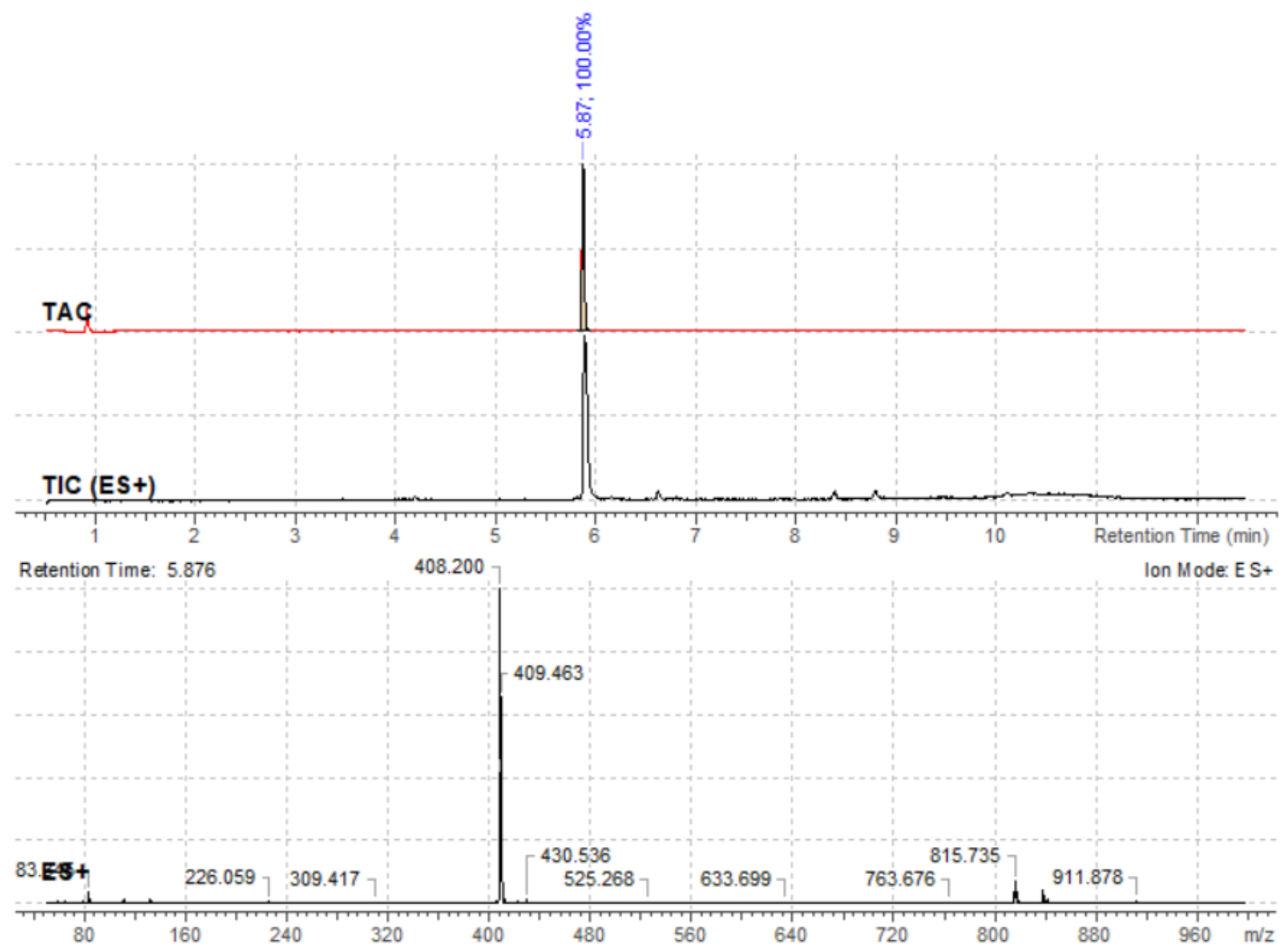


1-(2-Oxo-1-phenyl-2-(4-(3-(trifluoromethoxy)phenyl)piperazin-1-yl)ethyl)pyrrolidine-2,5-dione (25)

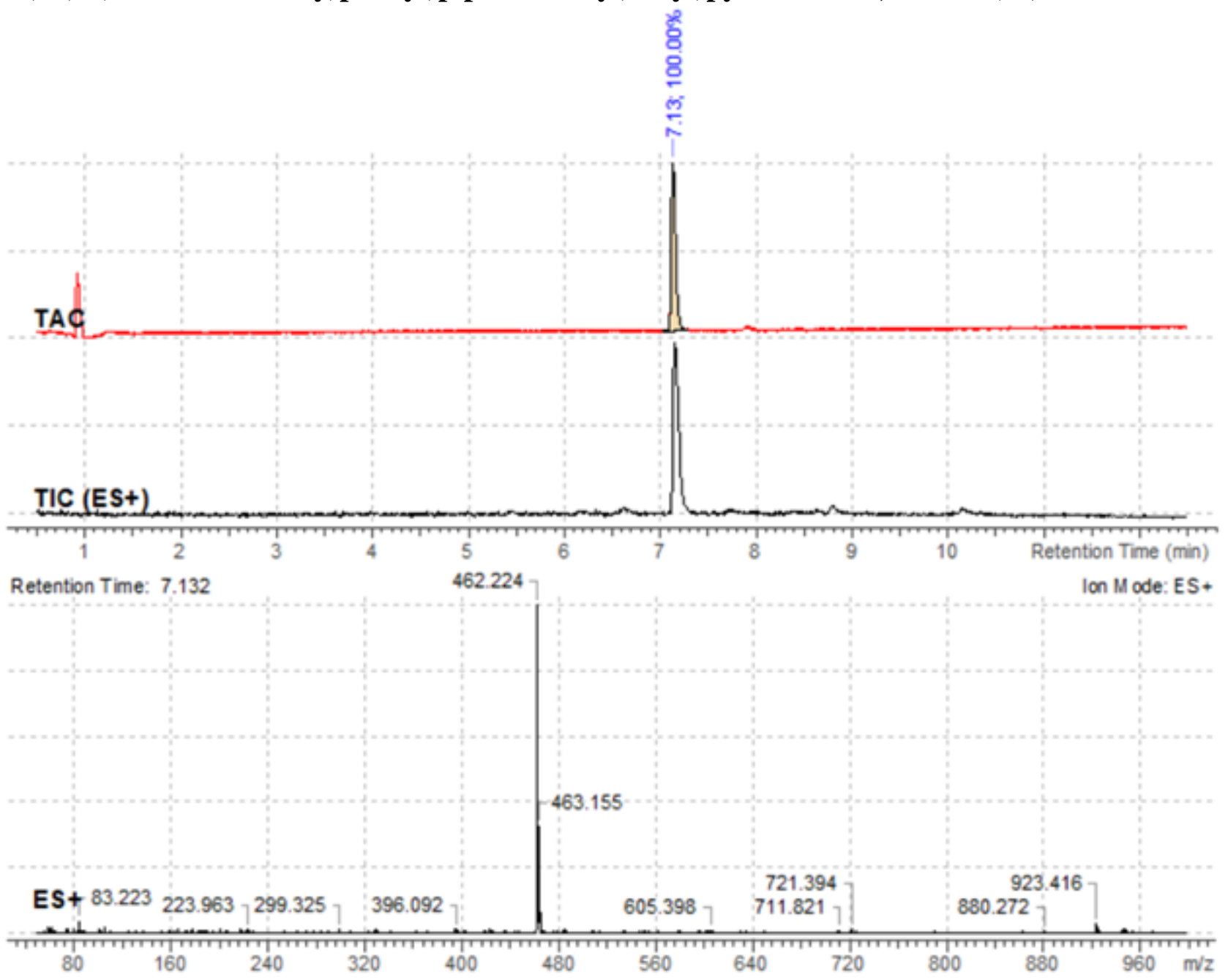


1-(2-(4-(3-(Methylthio)phenyl)piperazin-1-yl)-2-oxo-1-phenylethyl)pyrrolidine-2,5-dione (26).

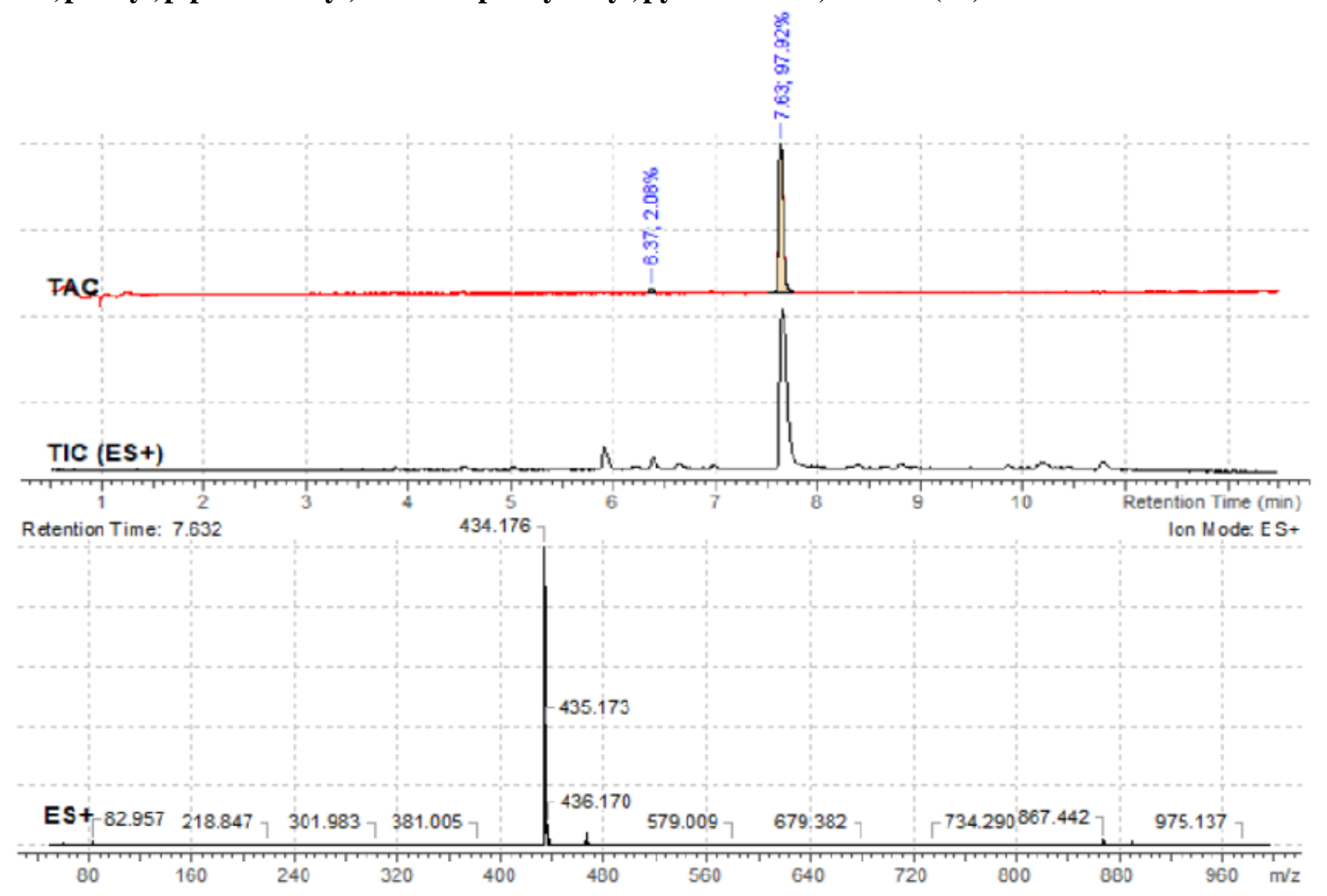


1-(2-Oxo-1-phenyl-2-(4-(3-((trifluoromethyl)thio)phenyl)piperazin-1-yl)ethyl)pyrrolidine-2,5-dione (27)

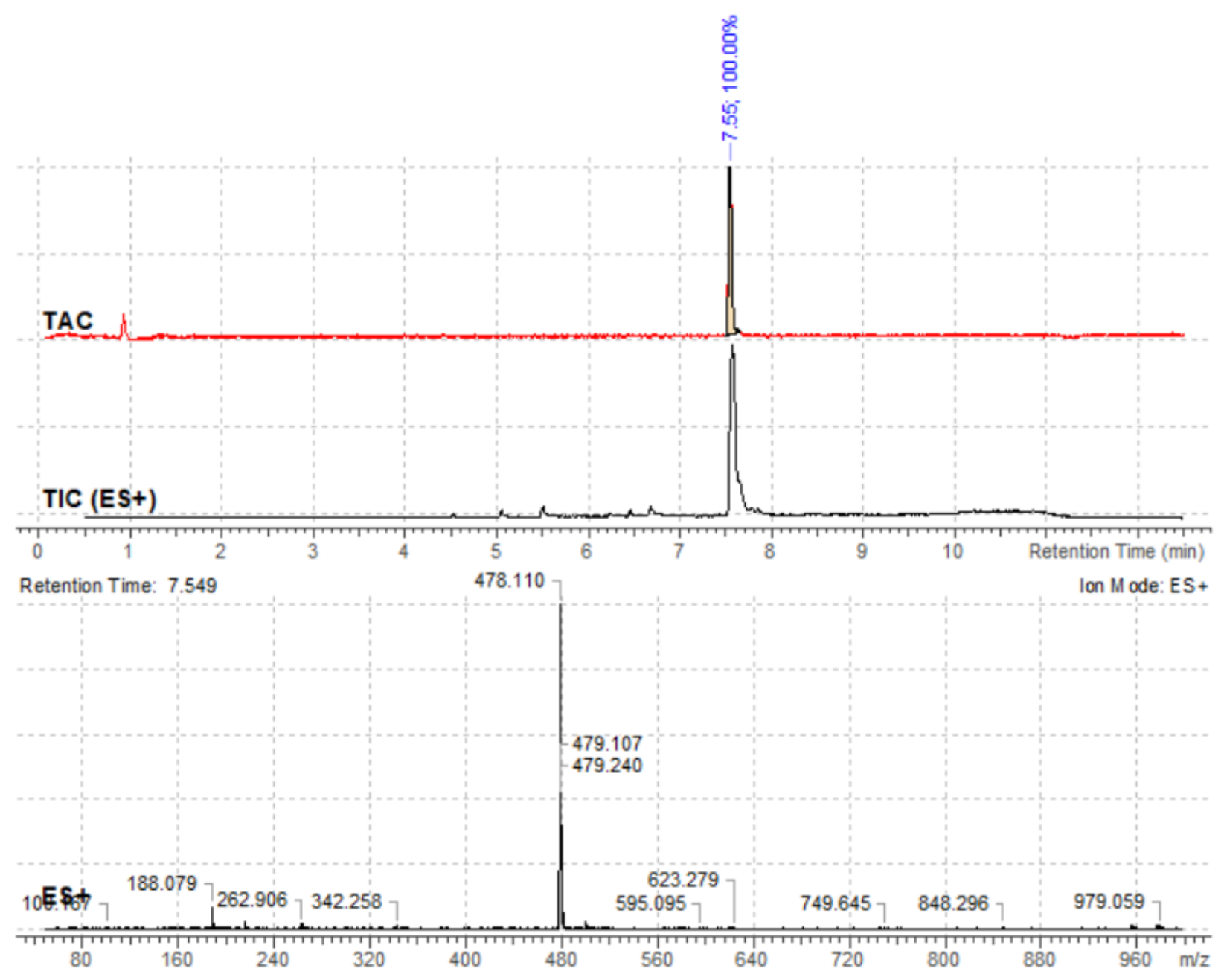


1-(2-(4-(3-(Difluoromethyl)phenyl)piperazin-1-yl)-2-oxo-1-phenylethyl)pyrrolidine-2,5-dione (28)

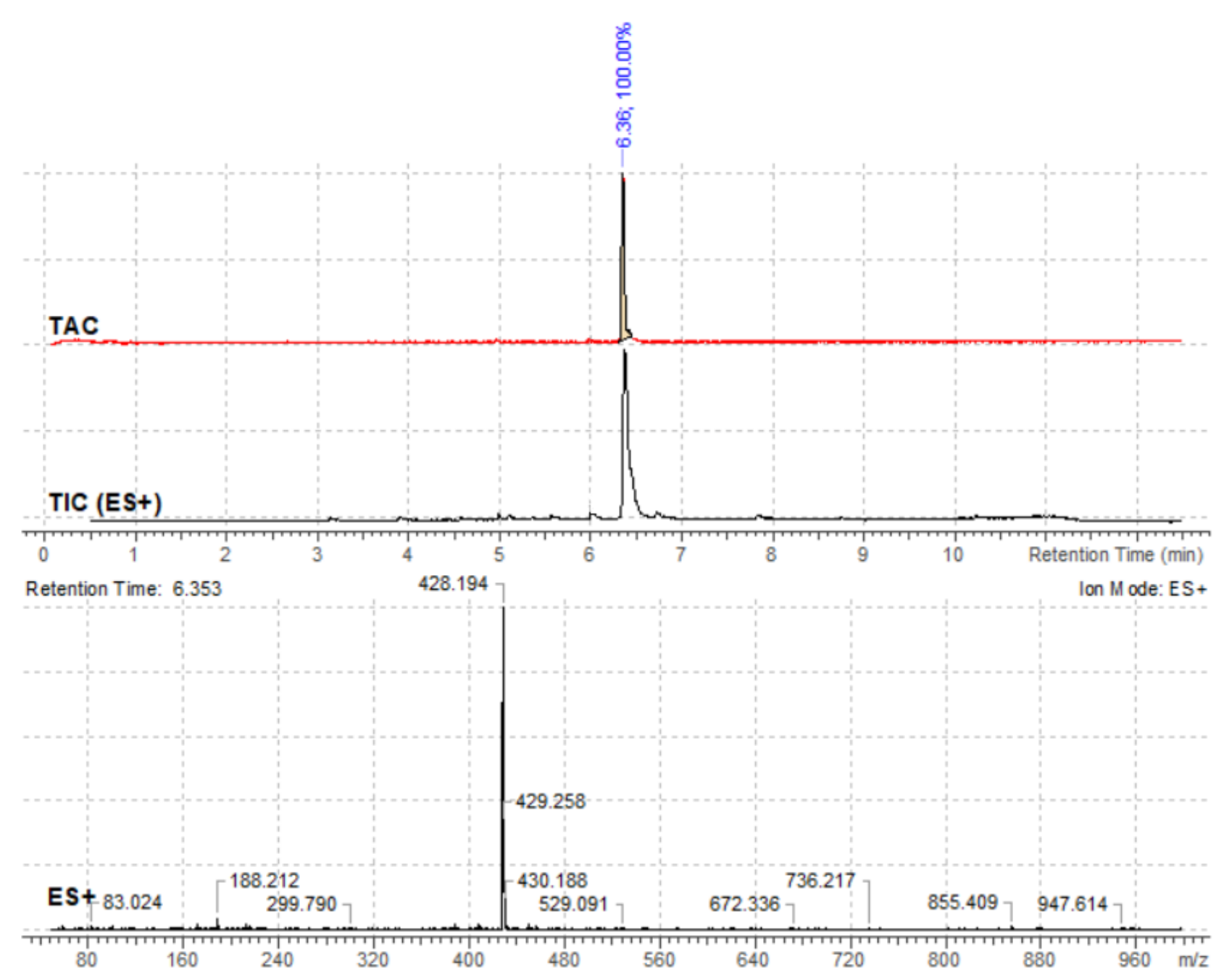


1-(2-(4-(3-Aminophenyl)piperazin-1-yl)-2-oxo-1-phenylethyl)pyrrolidine-2,5-dione (29).

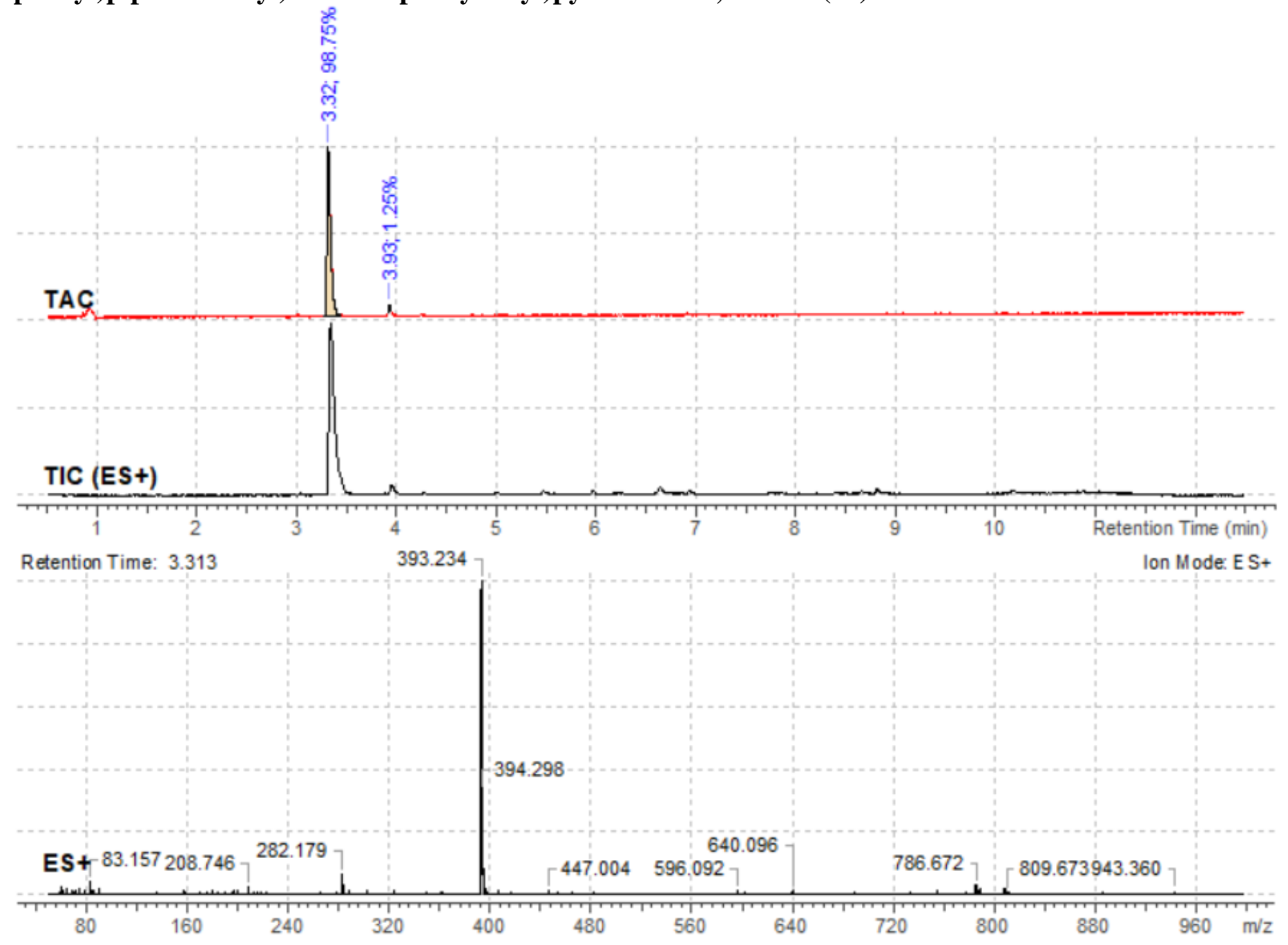


1-(2-(4-(3-Isopropylphenyl)piperazin-1-yl)-2-oxo-1-phenylethyl)pyrrolidine-2,5-dione (30).

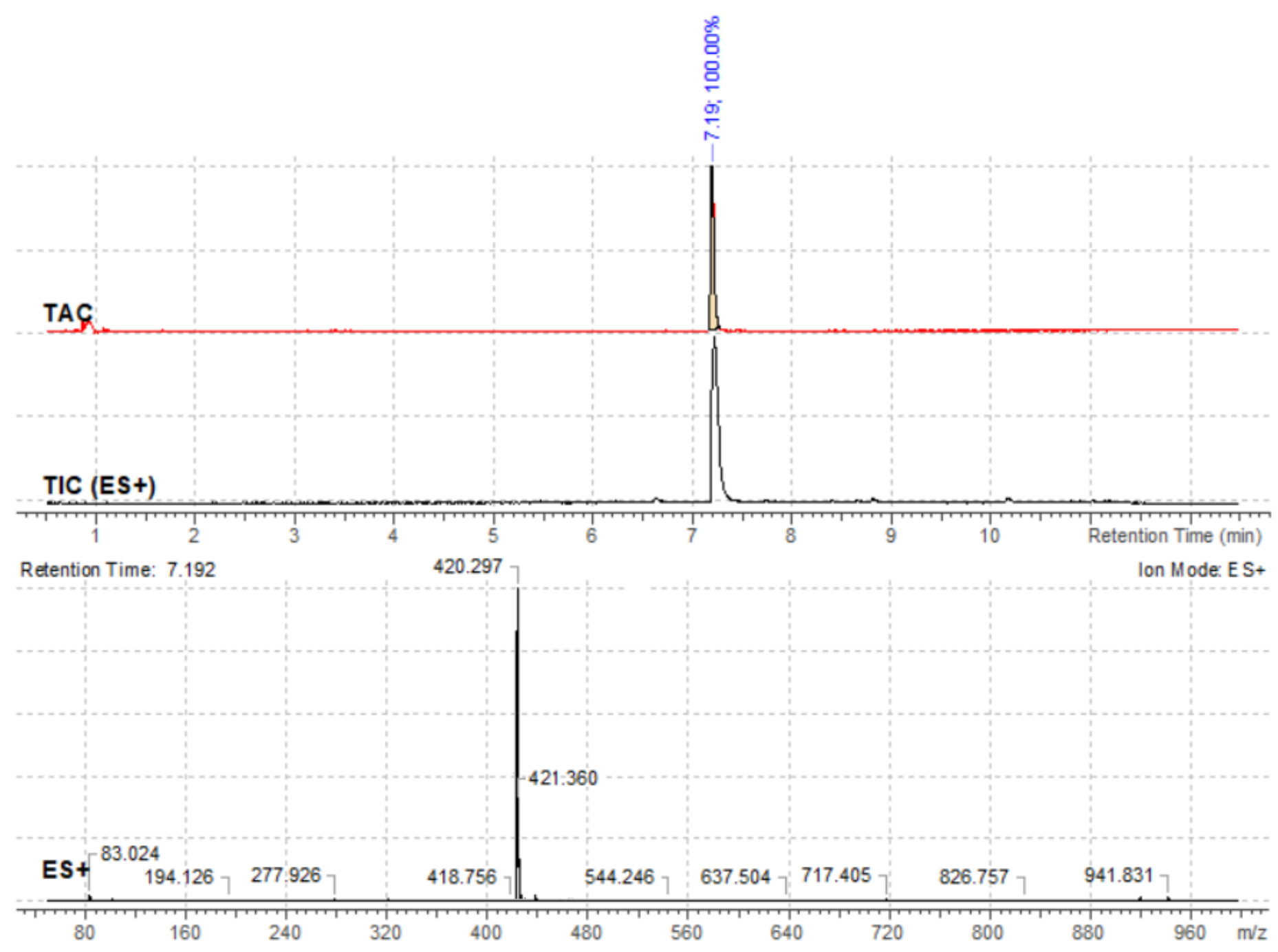


1-(2-(4-(3-(tert-Butyl)phenyl)piperazin-1-yl)-2-oxo-1-phenylethyl)pyrrolidine-2,5-dione (31).

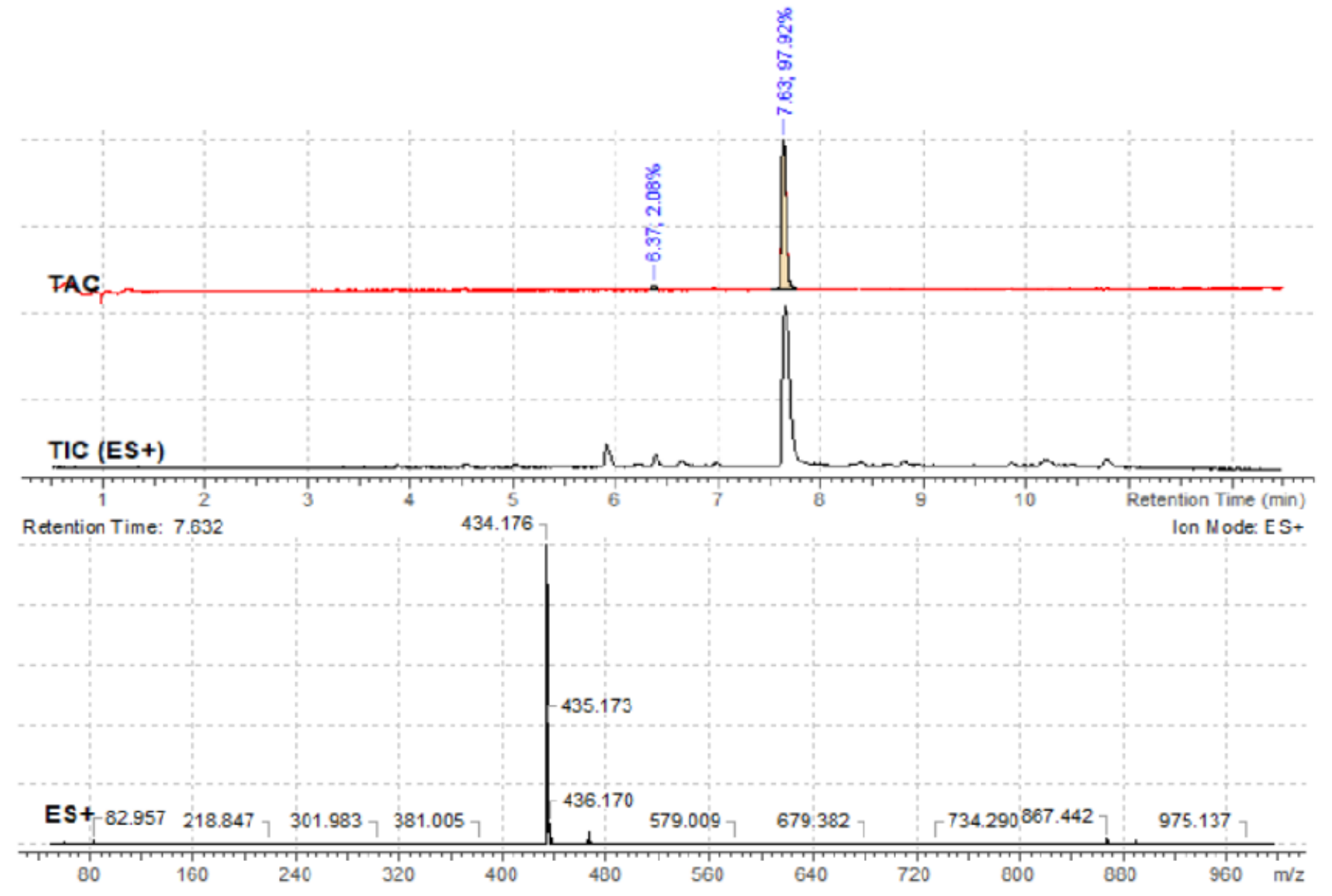


1-(2-(4-([1,1'-Biphenyl]-3-yl)piperazin-1-yl)-2-oxo-1-phenylethyl)pyrrolidine-2,5-dione (32)

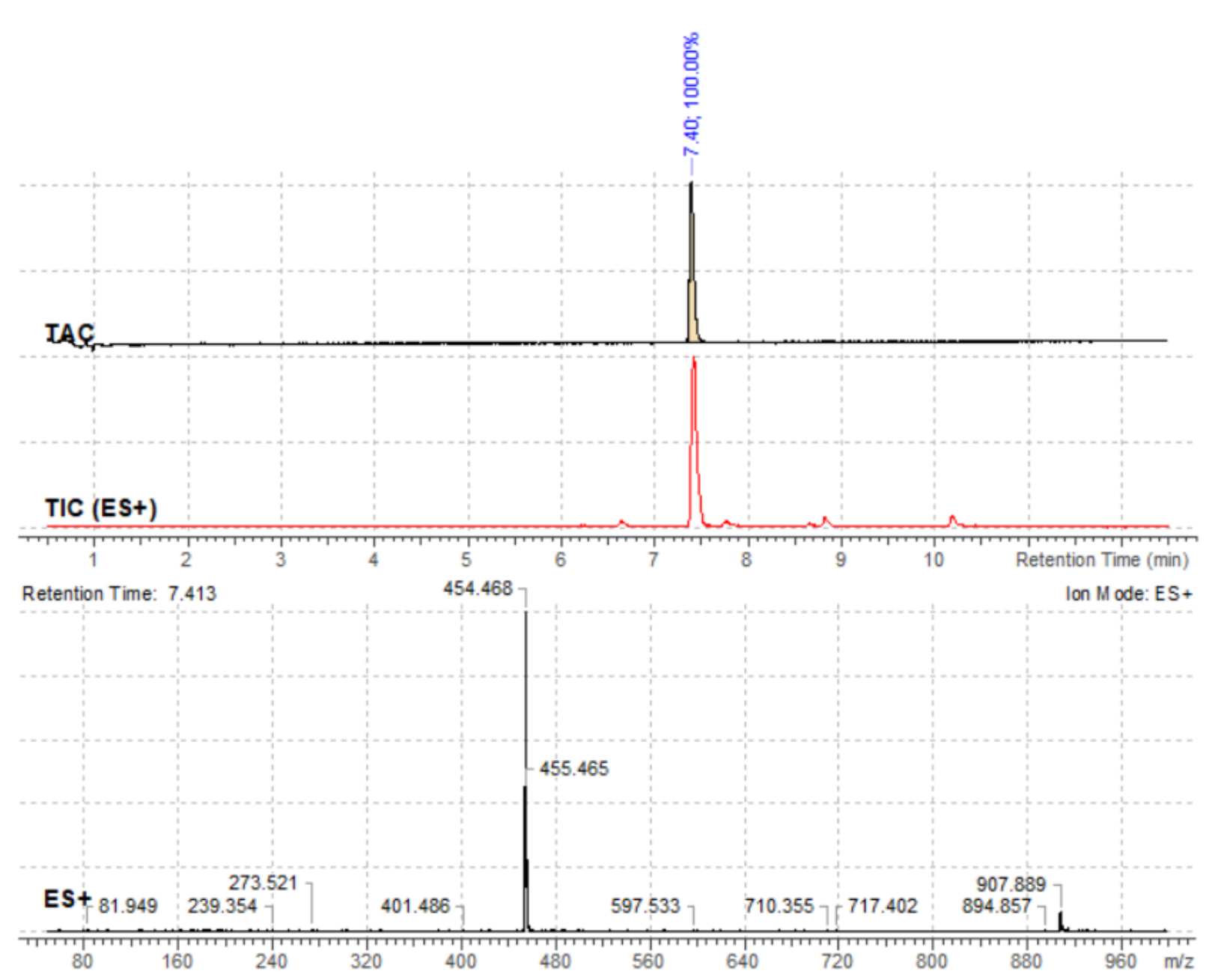


1-(2-(4-(3-(Benzyloxy)phenyl)piperazin-1-yl)-2-oxo-1-phenylethyl)pyrrolidine-2,5-dione (33).

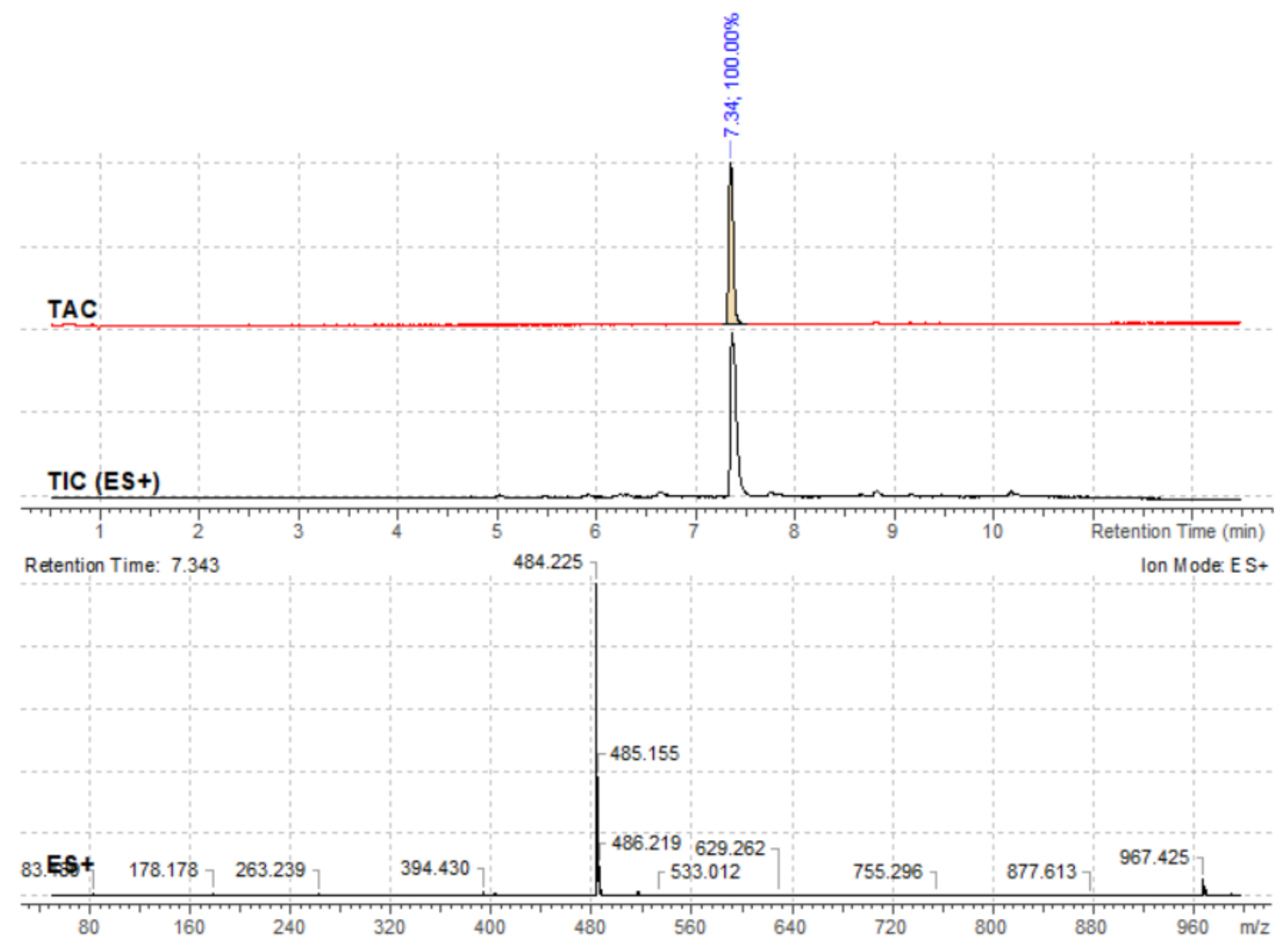


1-(1-(3-Fluorophenyl)-2-oxo-2-(4-(3-(trifluoromethyl)phenyl)piperazin-1-yl)ethyl)pyrrolidine-2,5-dione (34).

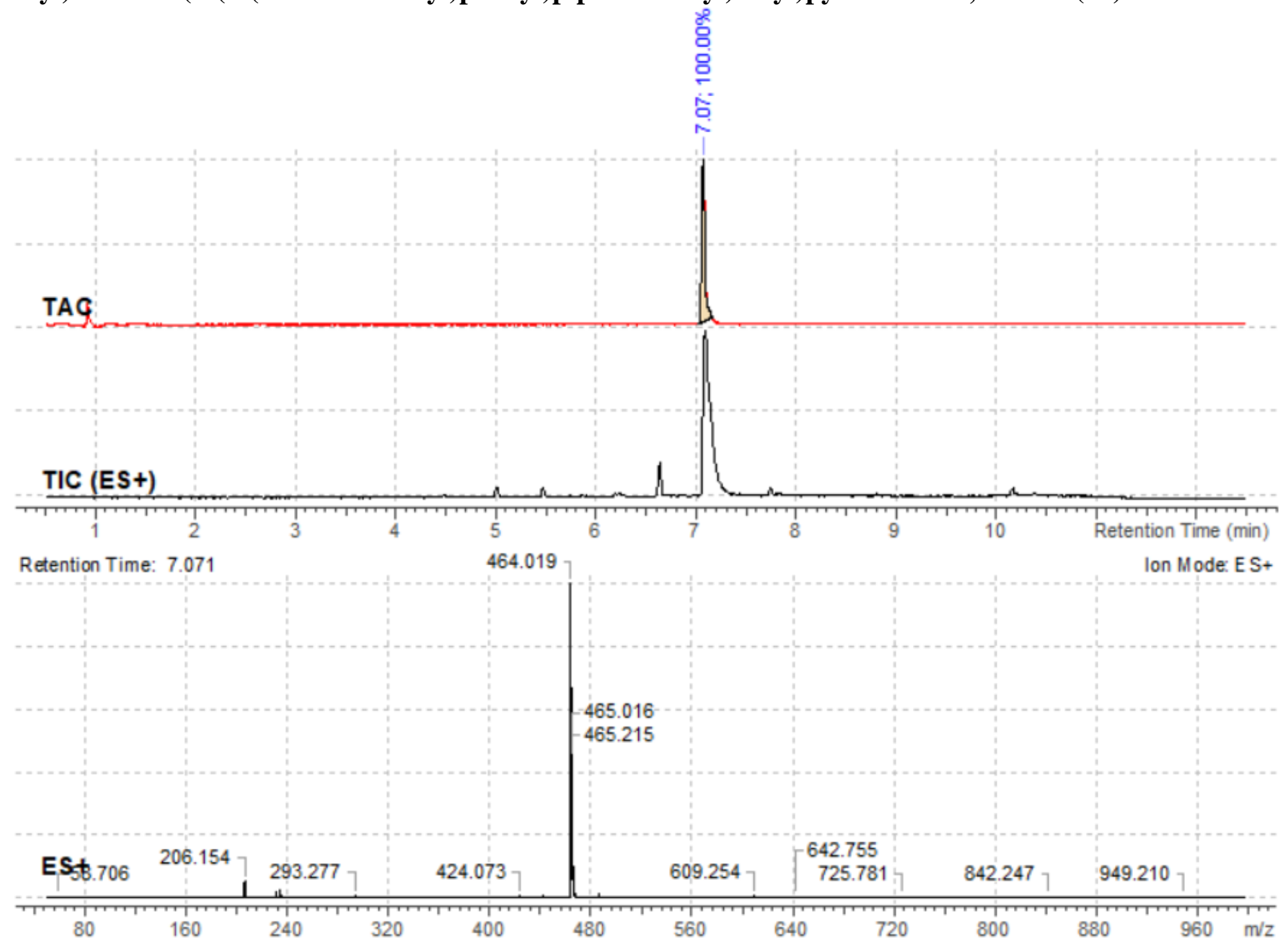


1-(1-(4-Fluorophenyl)-2-oxo-2-(4-(3-(trifluoromethyl)phenyl)piperazin-1-yl)ethyl)pyrrolidine-2,5-dione (35)

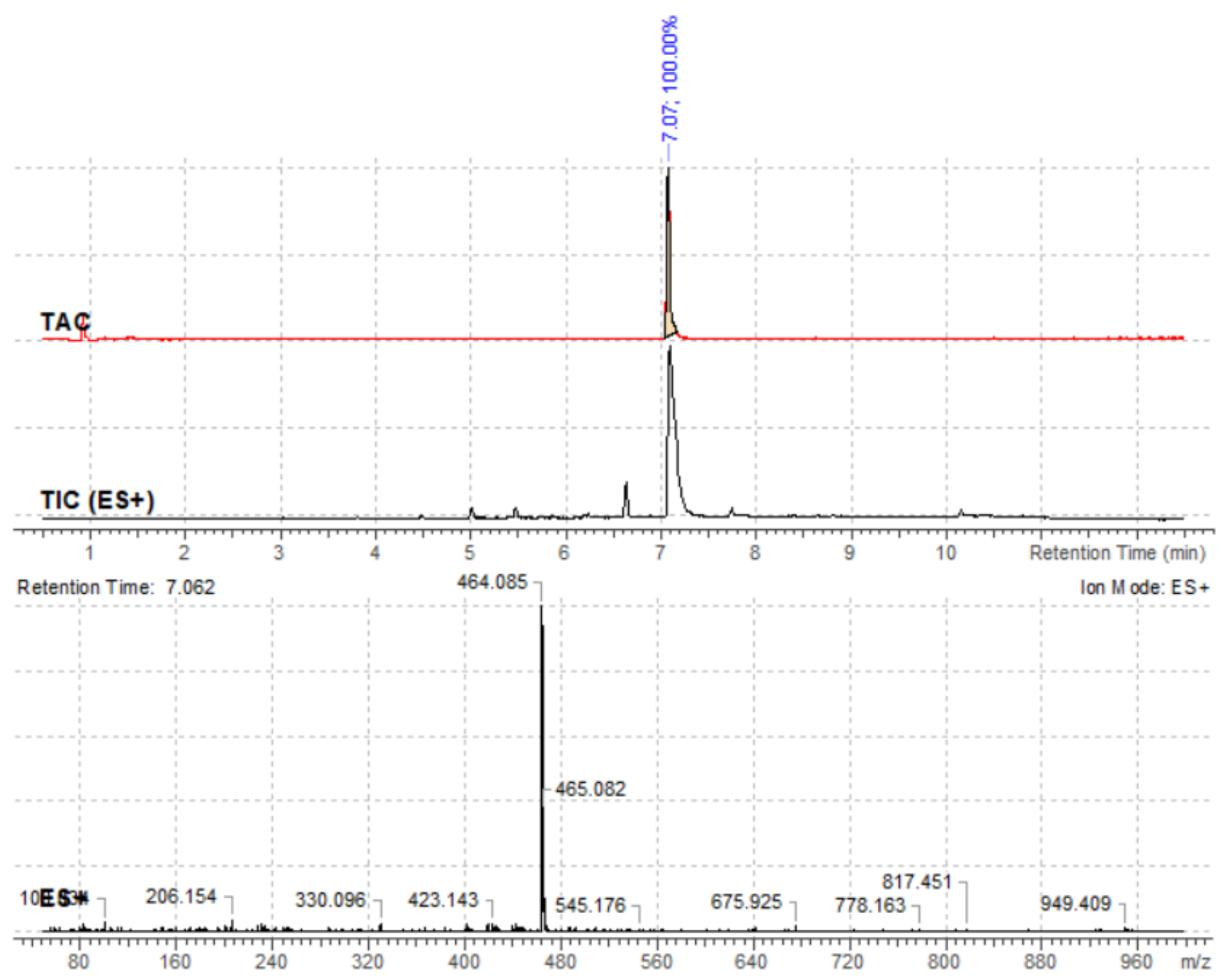


1-(1-(3,4-Difluorophenyl)-2-oxo-2-(4-(3-(trifluoromethyl)phenyl)piperazin-1-yl)ethyl)pyrrolidine-2,5-dione (36).

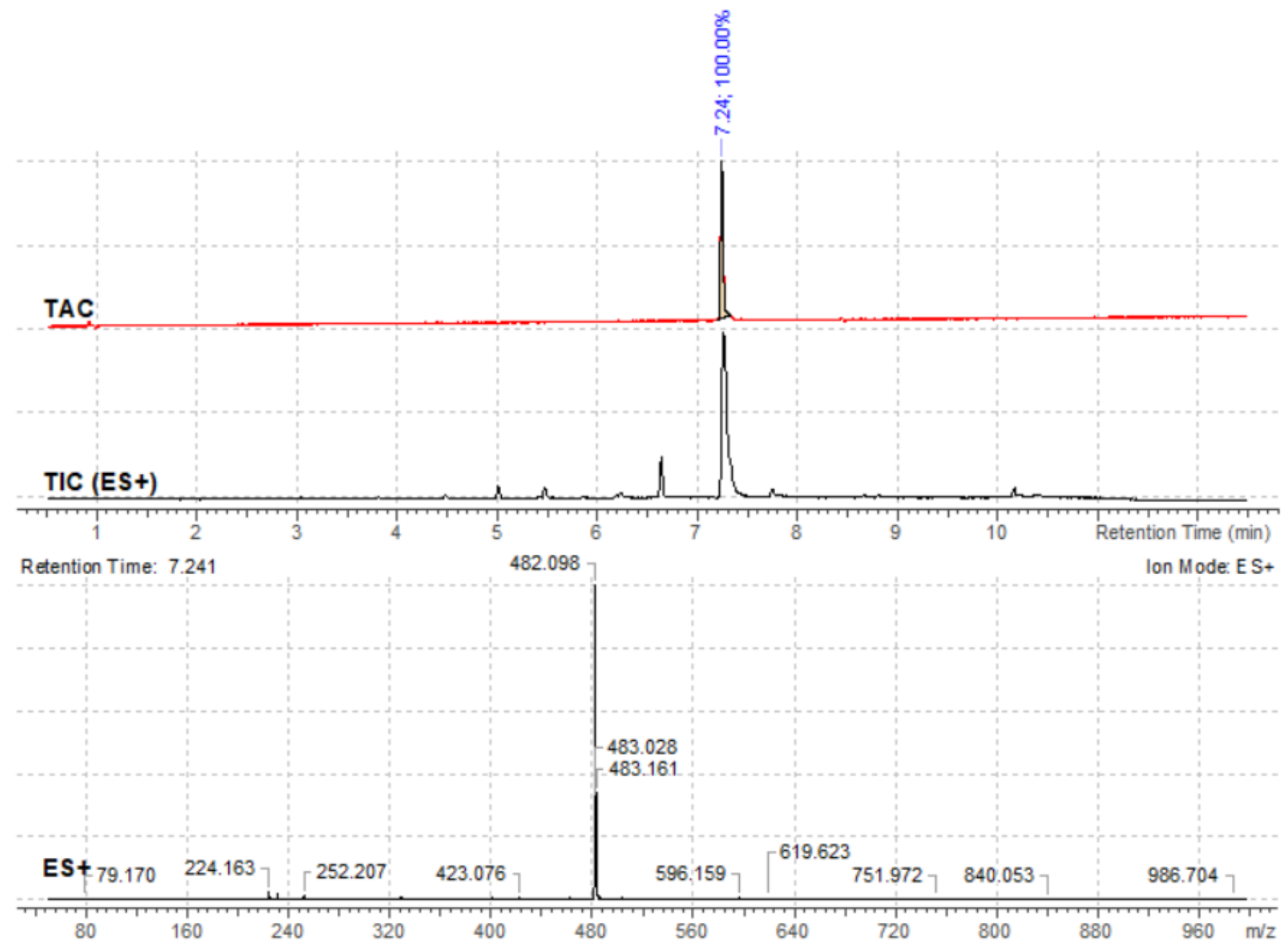


1-(1-Oxo-3-phenyl-1-(4-phenylpiperazin-1-yl)propan-2-yl)pyrrolidine-2,5-dione (37).

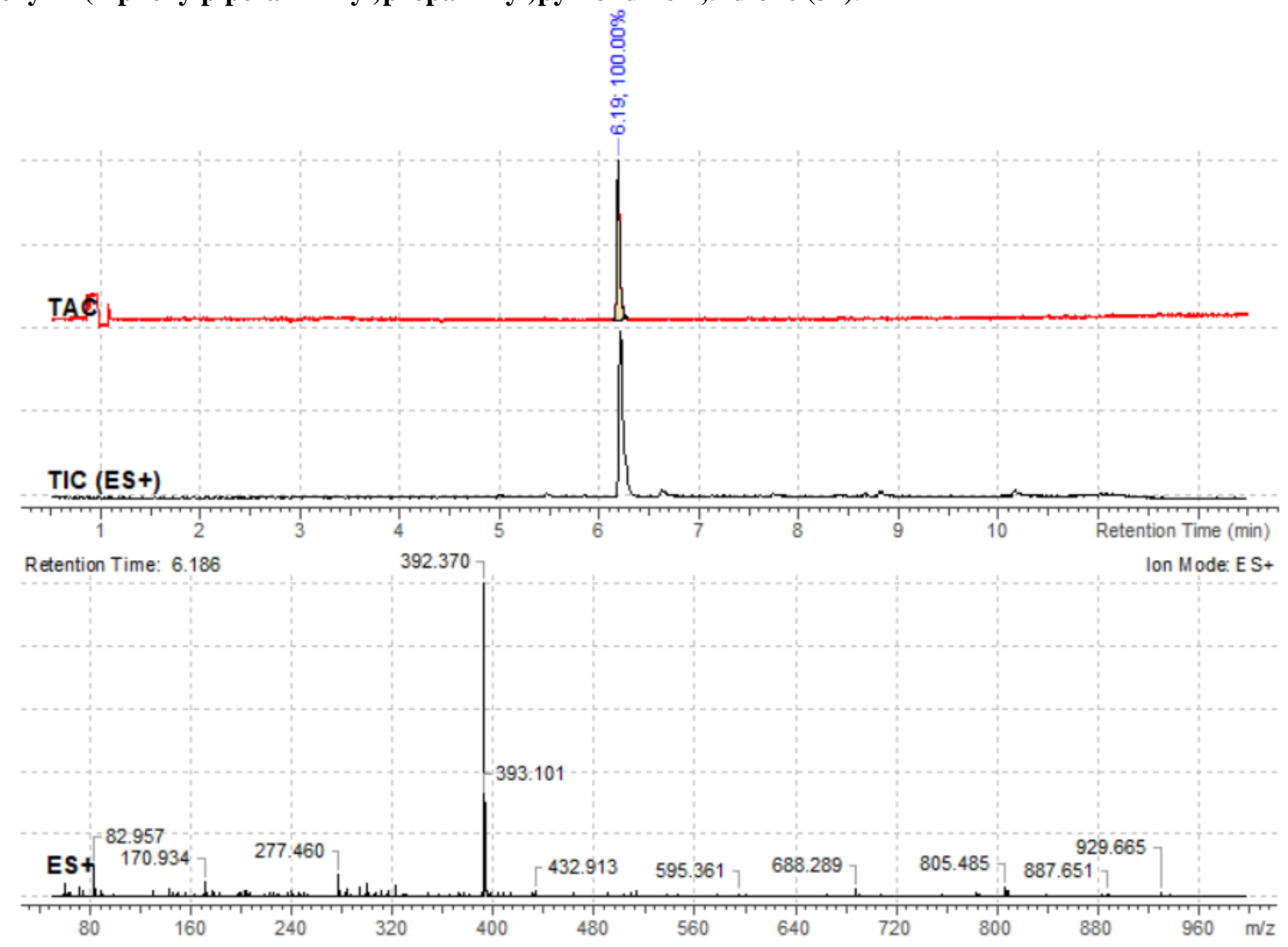


1-(1-(4-(3-Chlorophenyl)piperazin-1-yl)-1-oxo-3-phenylpropan-2-yl)pyrrolidine-2,5-dione (38).

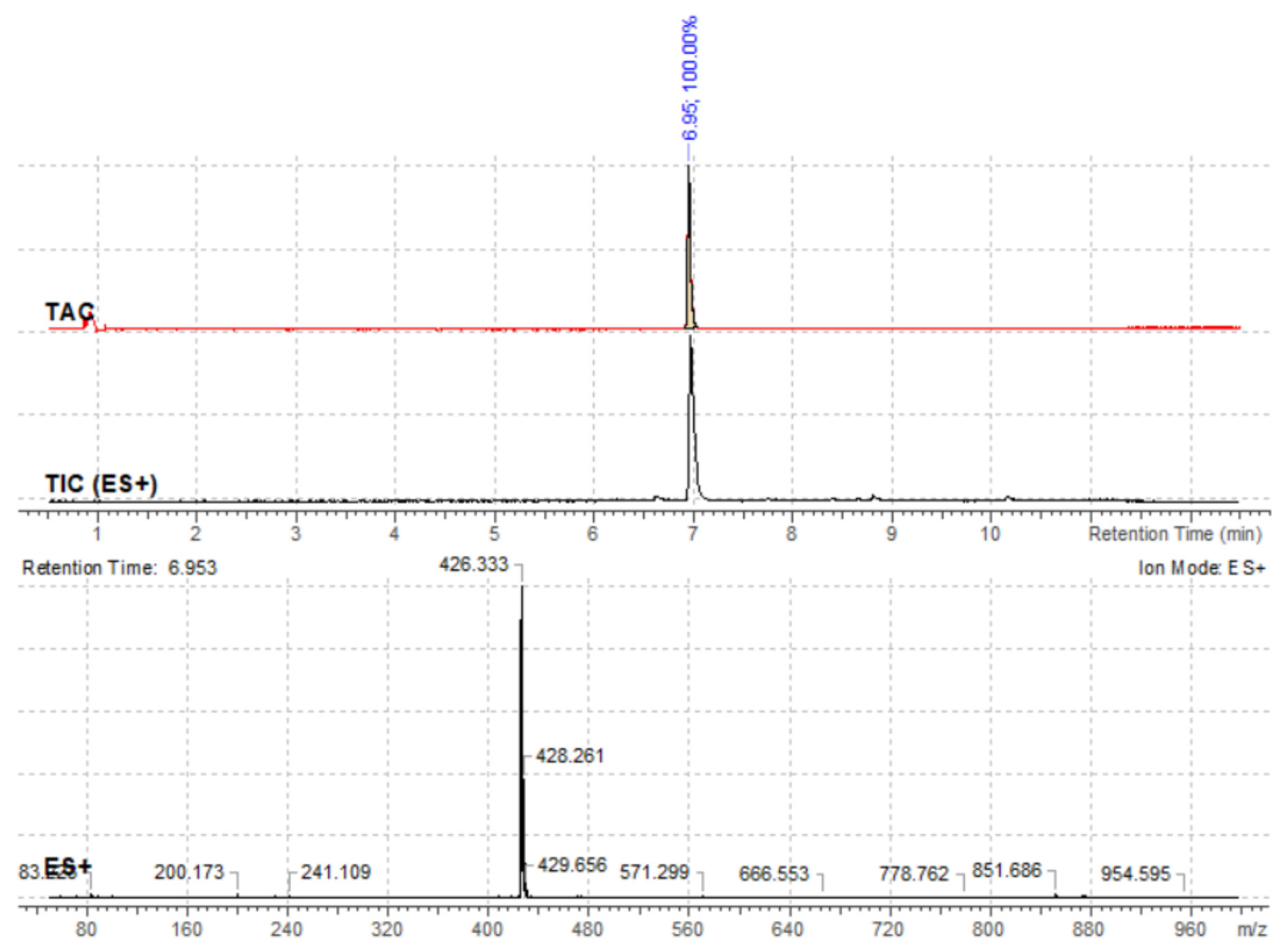


1-(1-Oxo-3-phenyl-1-(4-(m-tolyl)piperazin-1-yl)propan-2-yl)pyrrolidine-2,5-dione (39).

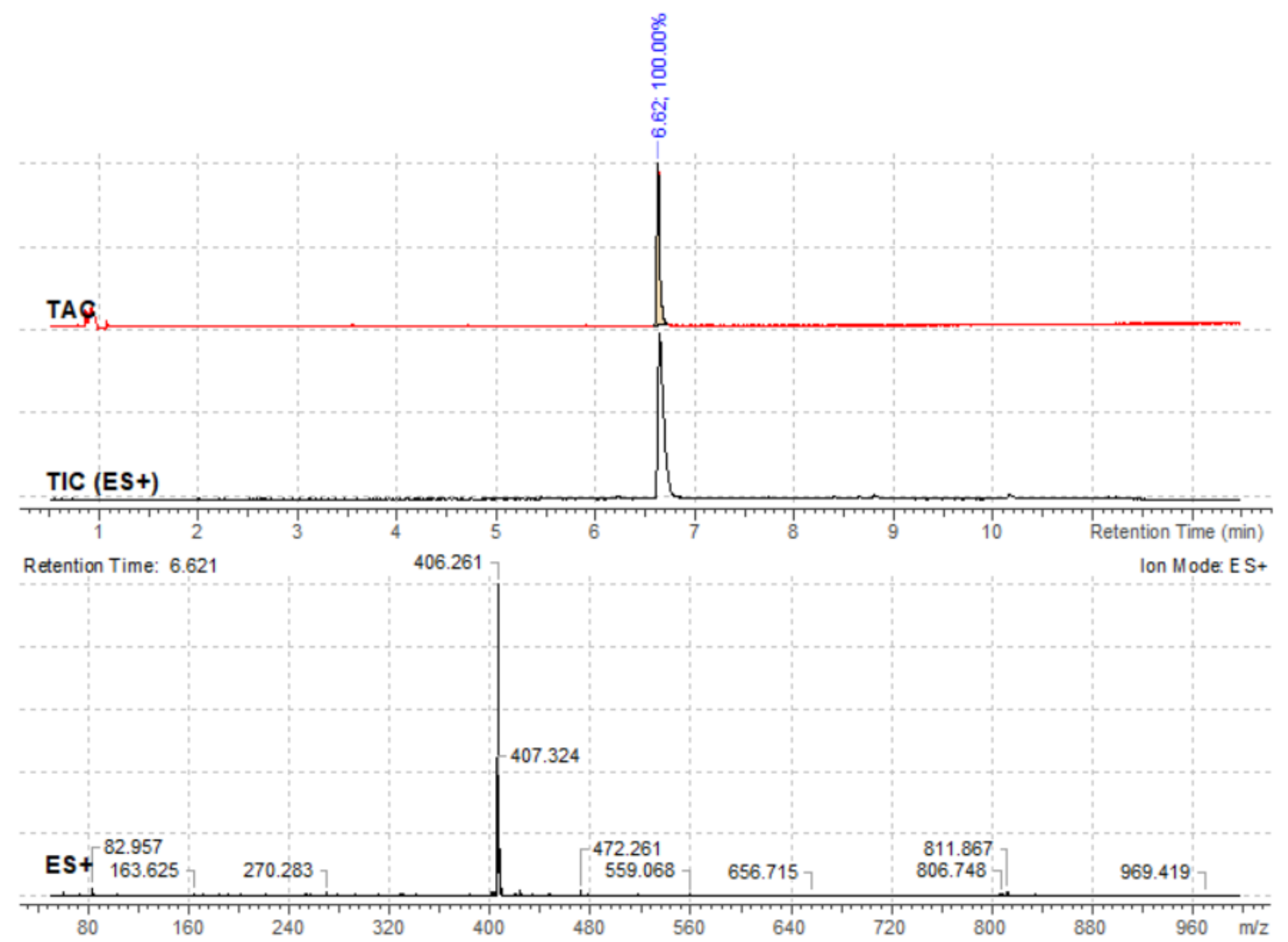


1-(1-Oxo-3-phenyl-1-(4-(3-(trifluoromethyl)phenyl)piperazin-1-yl)propan-2-yl)pyrrolidine-2,5-dione (40).

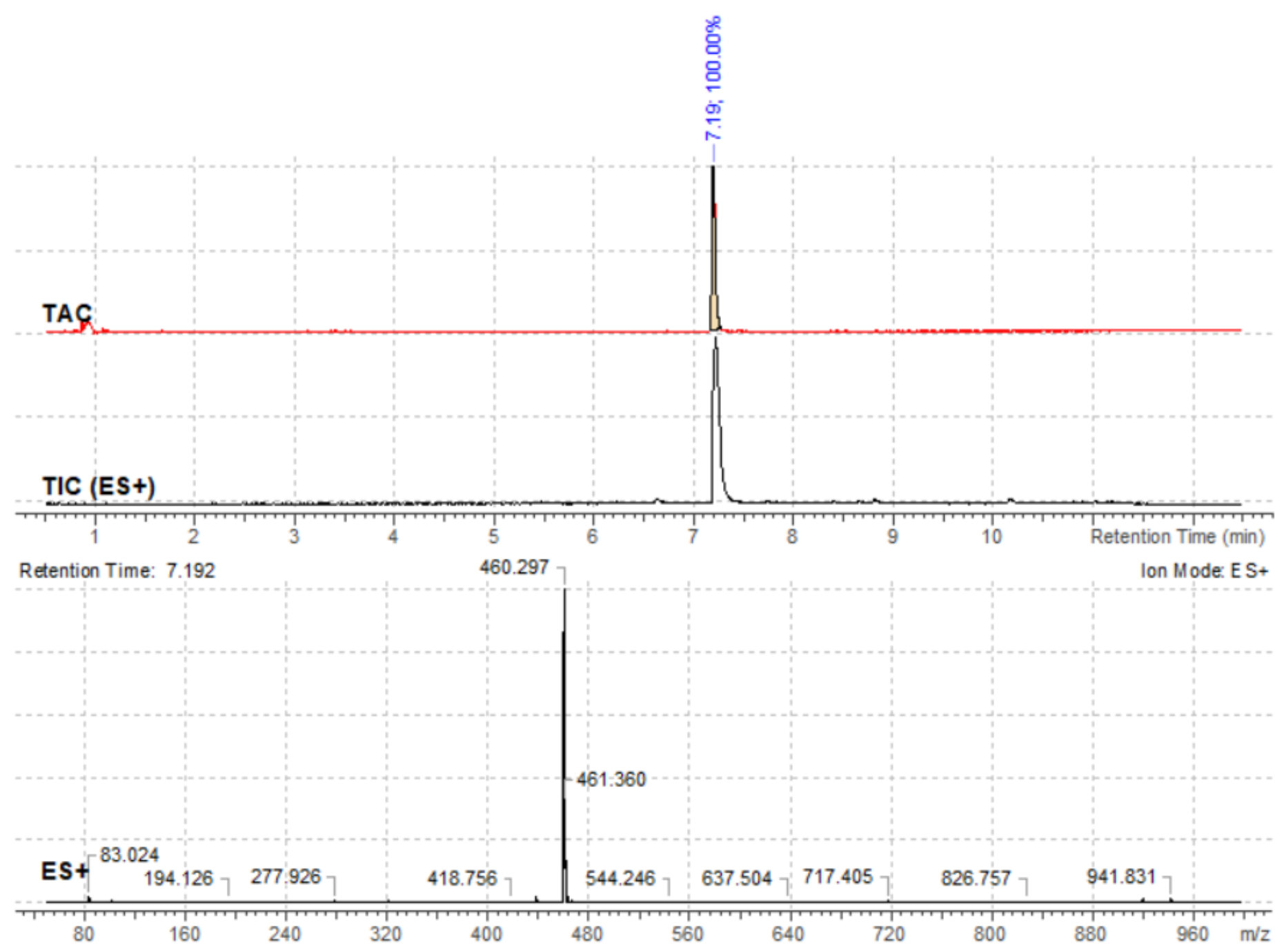


1-(1-Oxo-3-phenyl-1-(4-(3-(trifluoromethoxy)phenyl)piperazin-1-yl)propan-2-yl)pyrrolidine-2,5-dione (41).

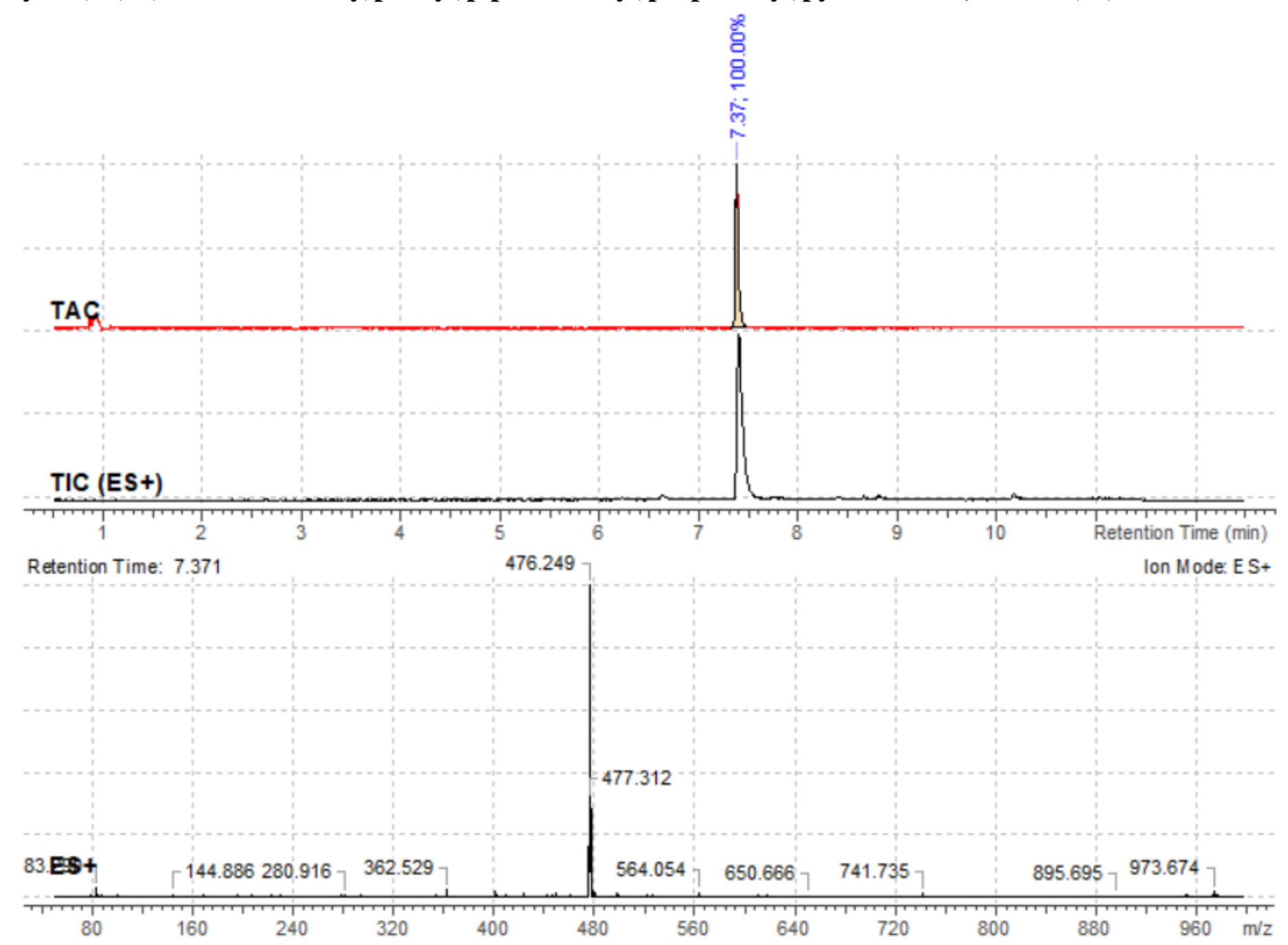


1-(2-(4-(2,3-Dichlorophenyl)piperazin-1-yl)-2-oxo-1-phenylethyl)pyrrolidine-2,5-dione (42).

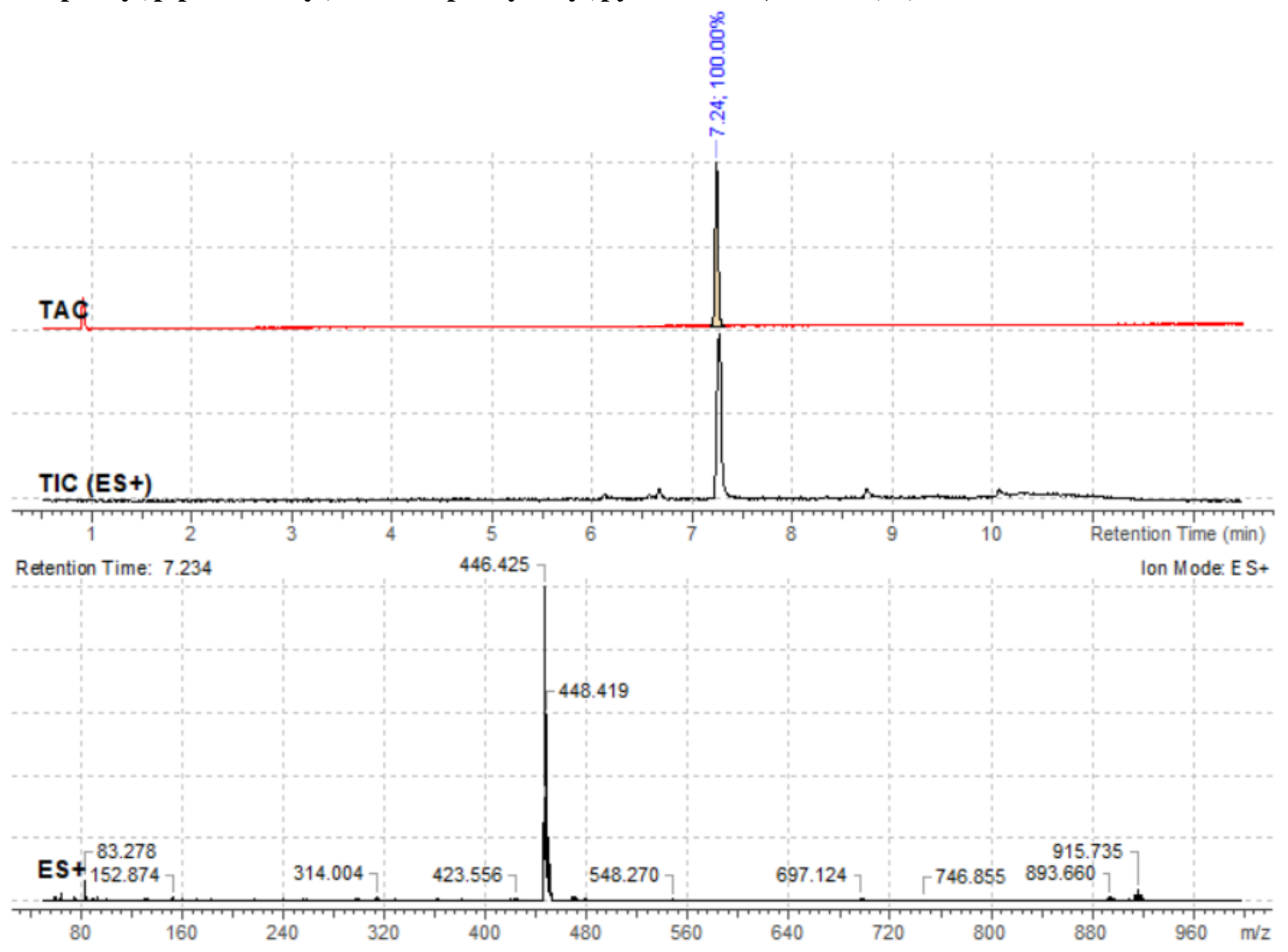


1-(2-(4-(3,4-Dichlorophenyl)piperazin-1-yl)-2-oxo-1-phenylethyl)pyrrolidine-2,5-dione (43).

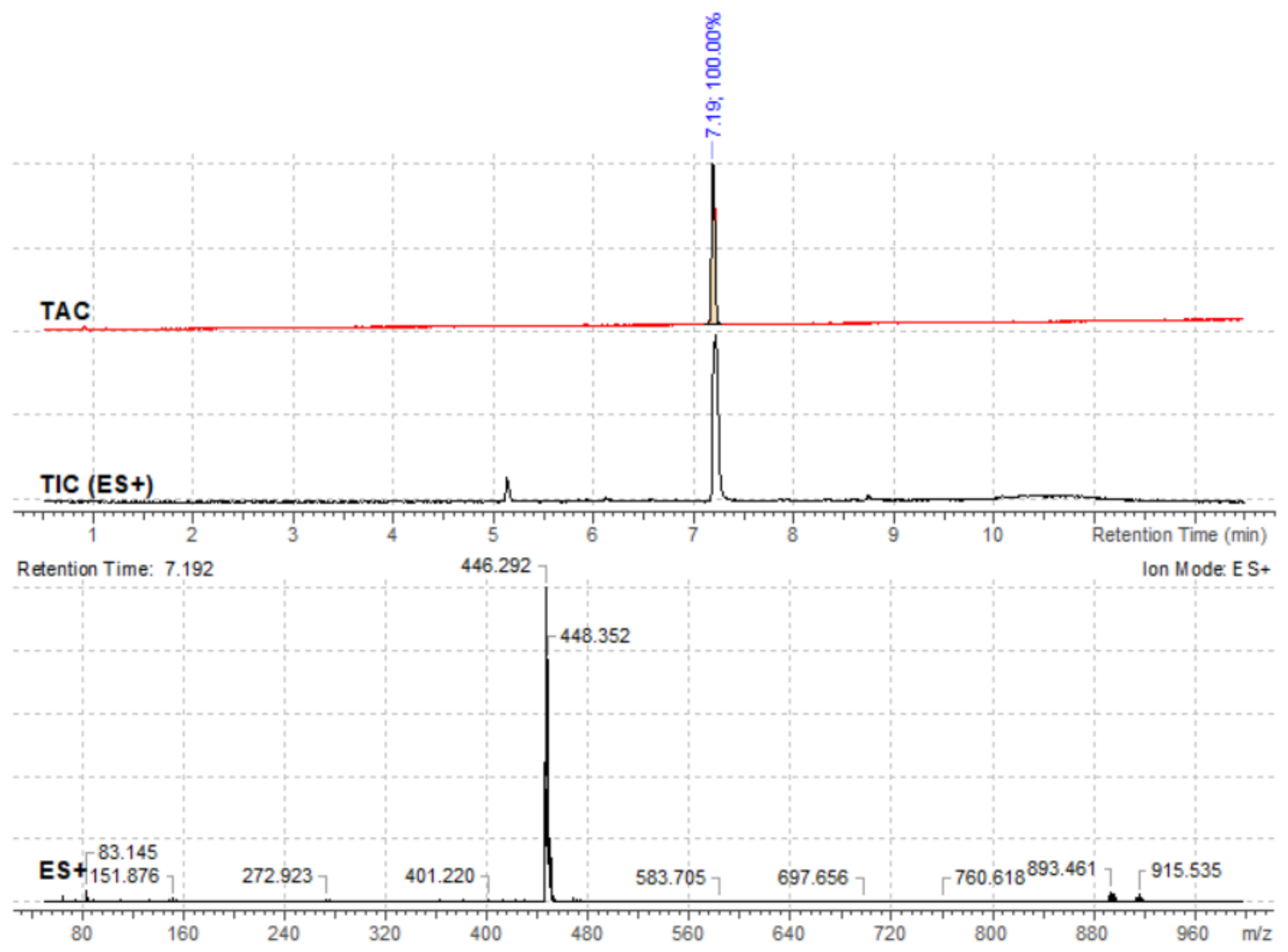


1-(2-(4-(3,5-Dichlorophenyl)piperazin-1-yl)-2-oxo-1-phenylethyl)pyrrolidine-2,5-dione (44)

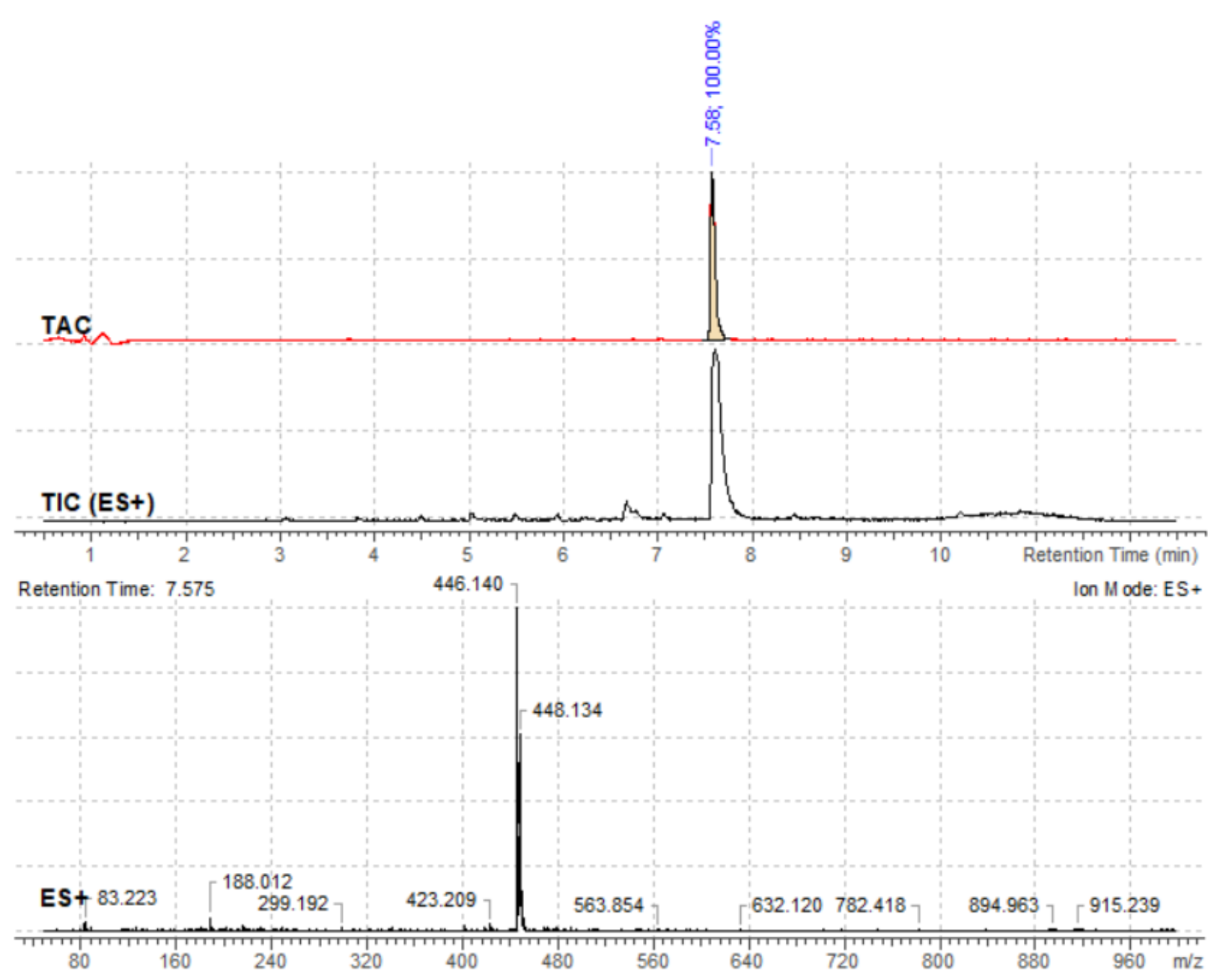


1-(2-(4-(3,5-bis(trifluoromethyl)phenyl)piperazin-1-yl)-2-oxo-1-phenylethyl)pyrrolidine-2,5-dione (45).

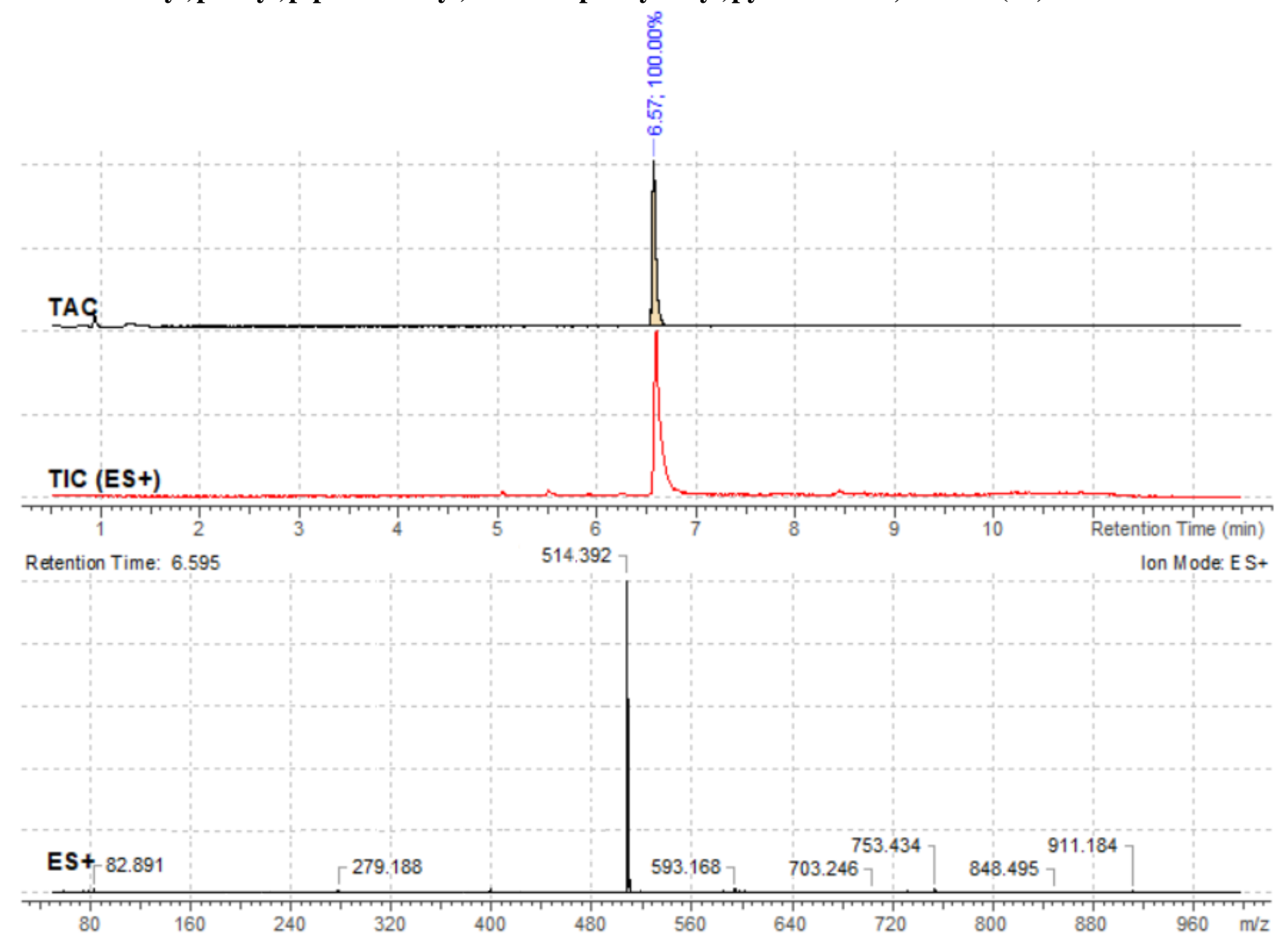


1-(2-(4-(3,5-bis((trifluoromethyl)thio)phenyl)piperazin-1-yl)-2-oxo-1-phenylethyl)pyrrolidine-2,5-dione (46).

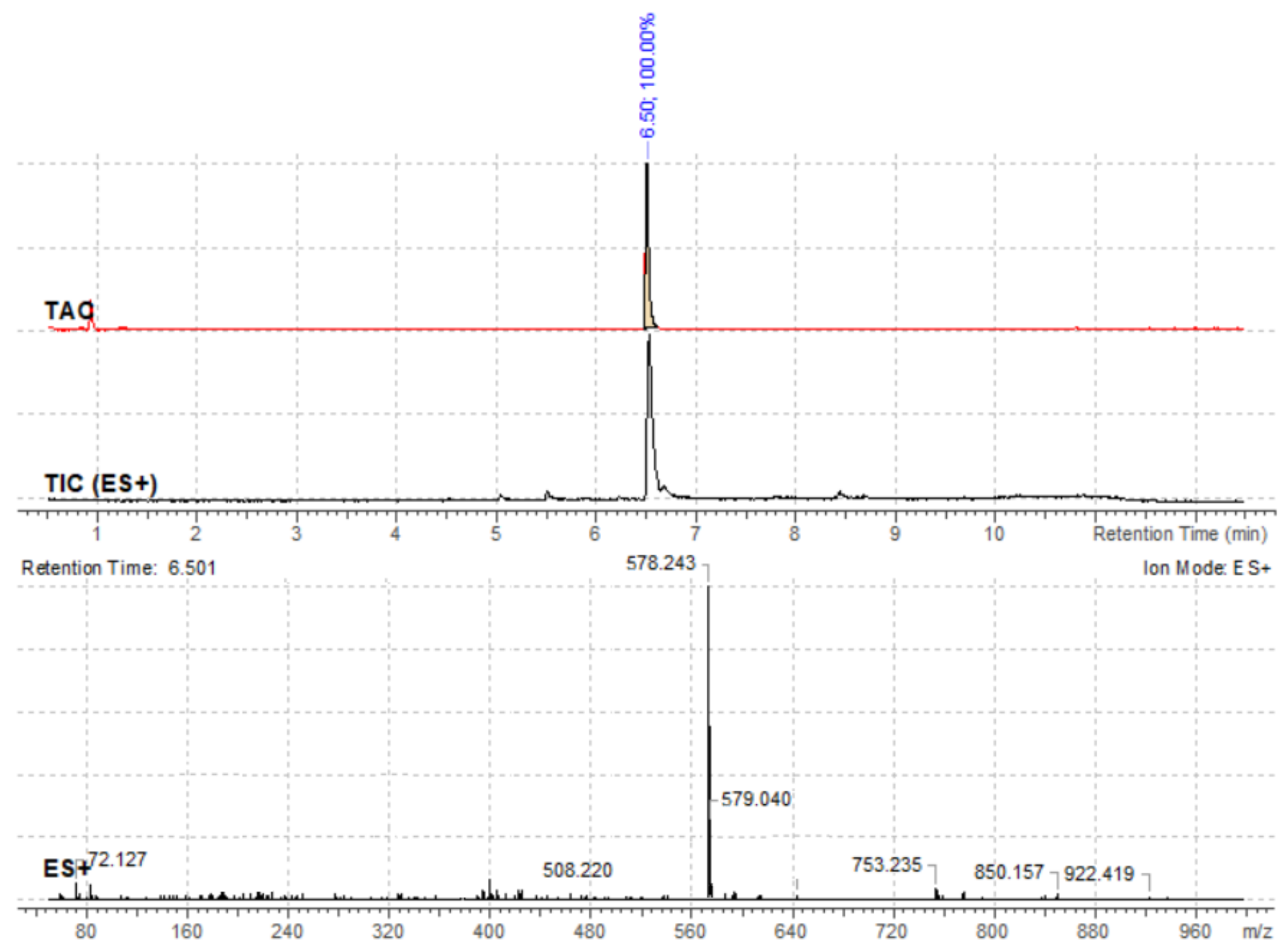


1-(2-(4-(3-chloro-5-(trifluoromethyl)phenyl)piperazin-1-yl)-2-oxo-1-phenylethyl)pyrrolidine-2,5-dione (47).

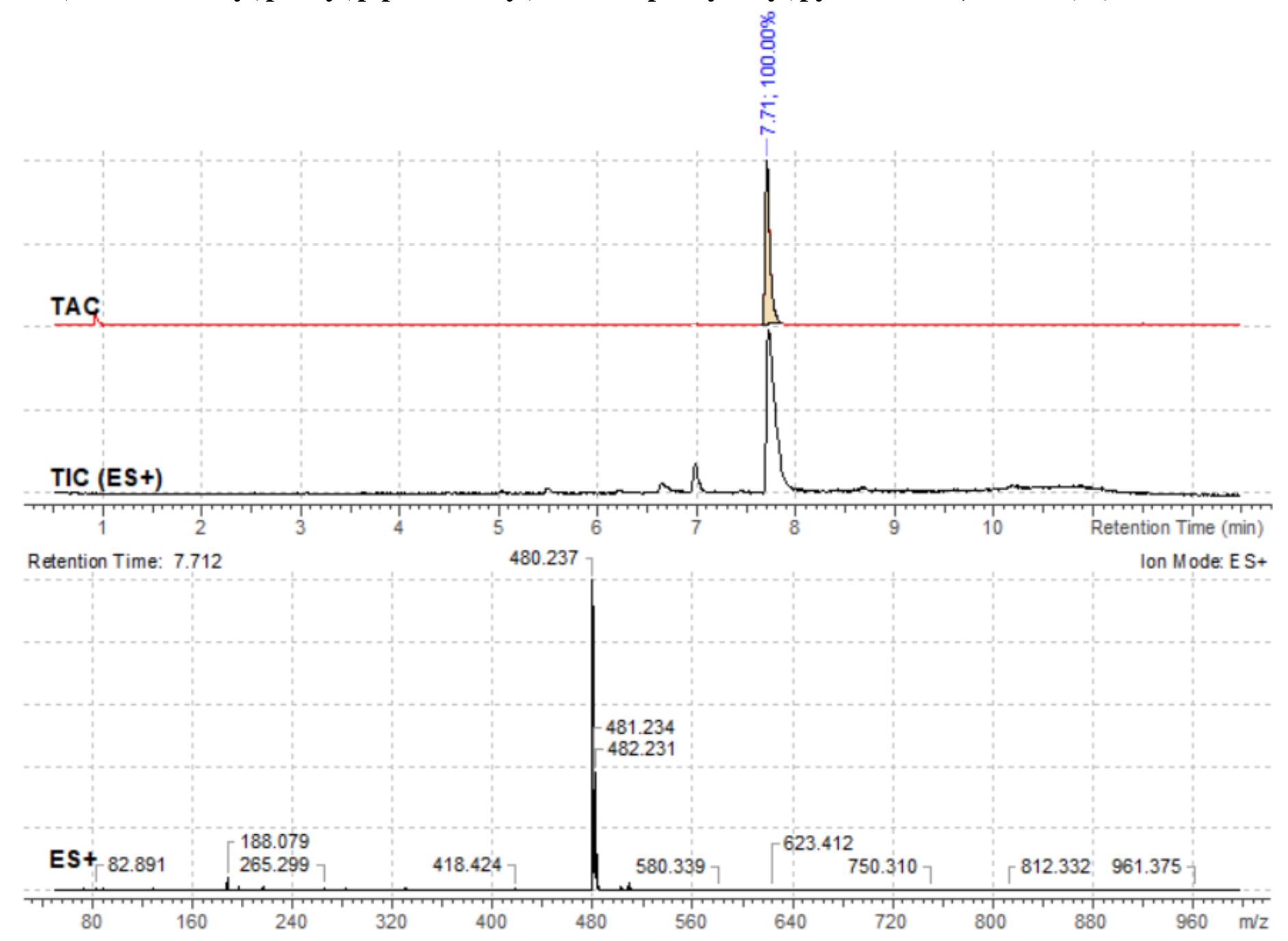


1-(2-Oxo-1-phenyl-2-(4-(3-(trifluoromethyl)phenyl)piperidin-1-yl)ethyl)pyrrolidine-2,5-dione (48)
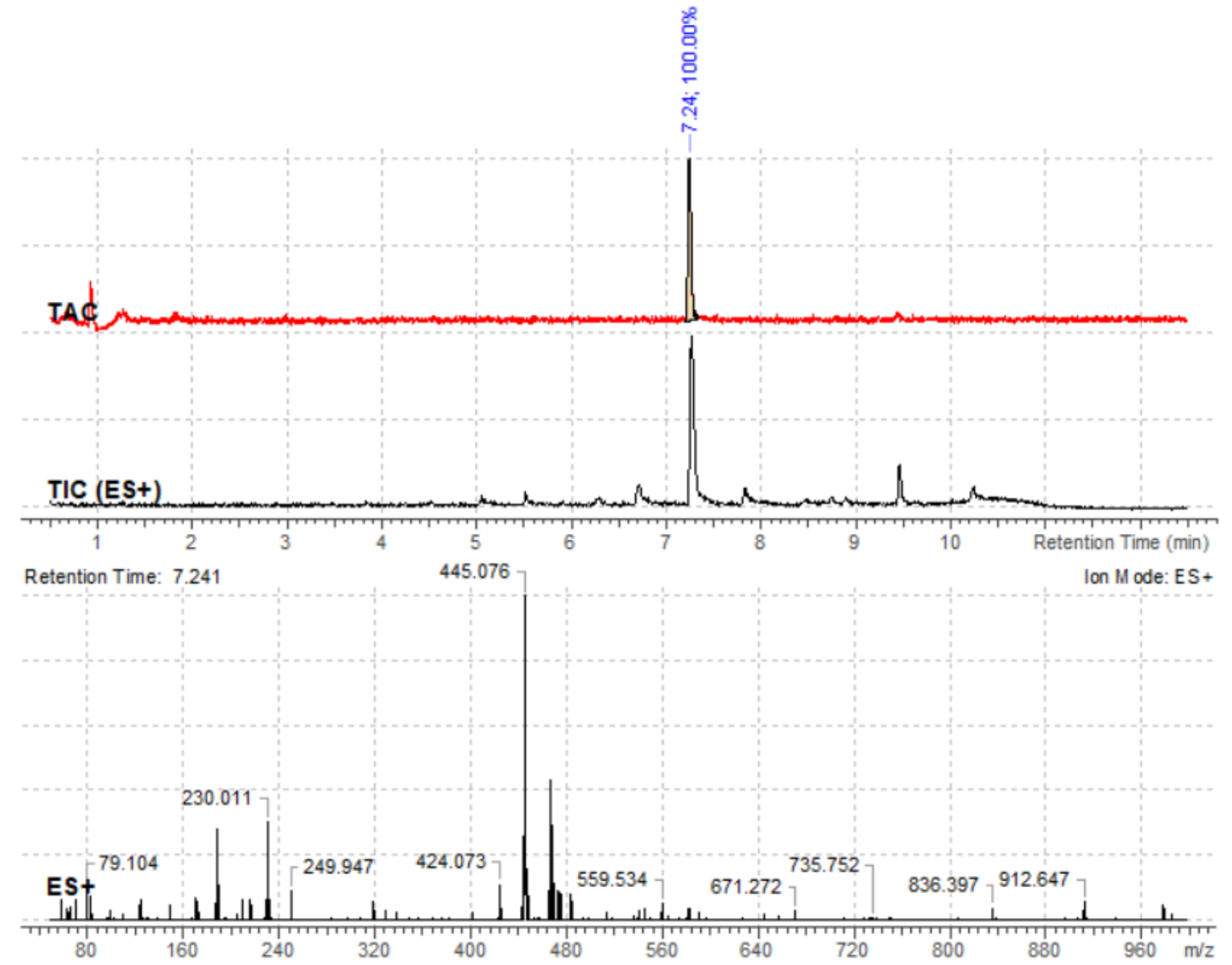
1-(2-Oxo-1-phenyl-2-(4-(3-(trifluoromethyl)phenyl)-1,4-diazepan-1-yl)ethyl)pyrrolidine-2,5-dione (49).

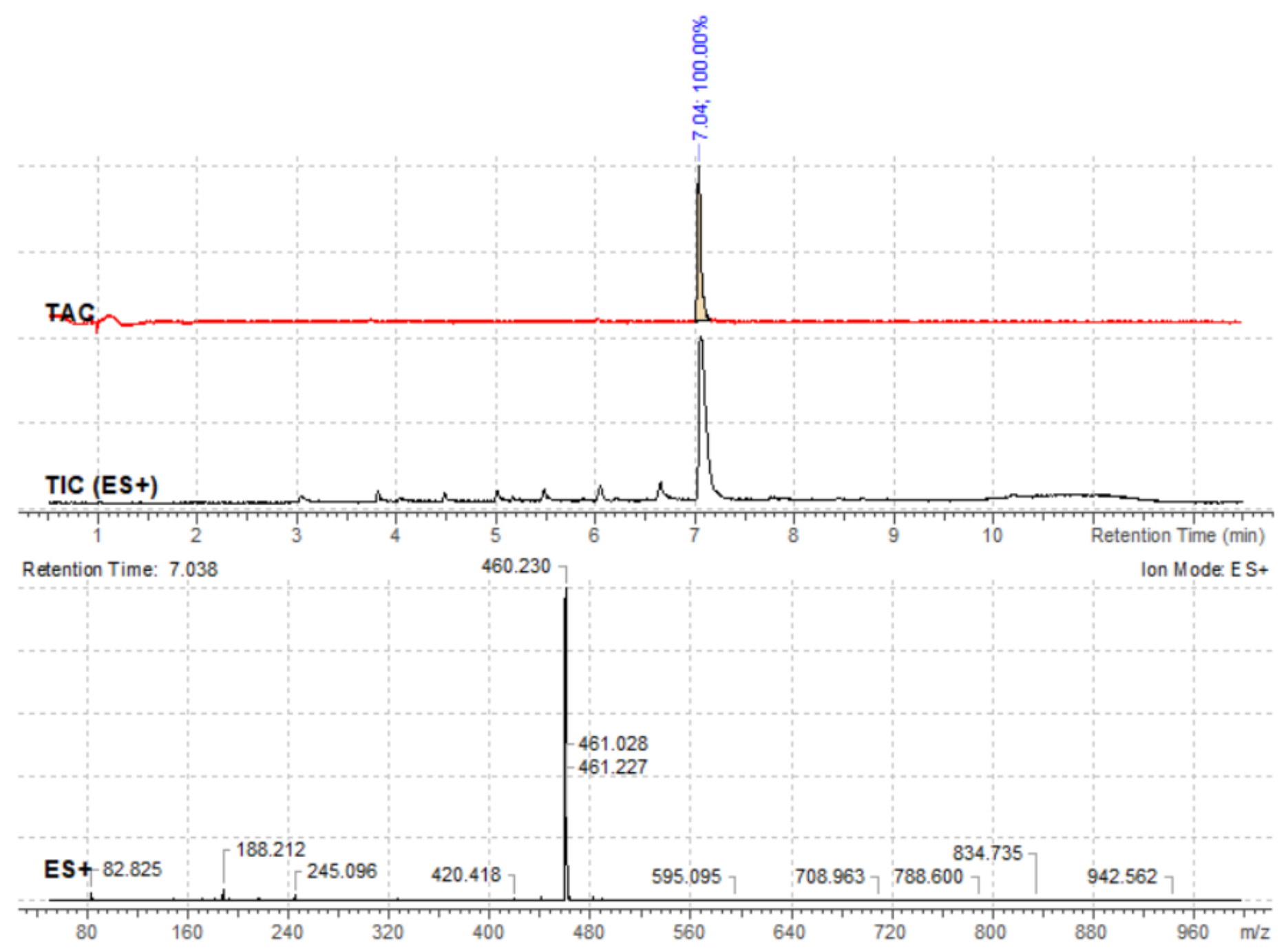


1-(3-(4-(3-(Trifluoromethyl)phenyl)piperazine-1-carbonyl)phenyl)pyrrolidine-2,5-dione (52).

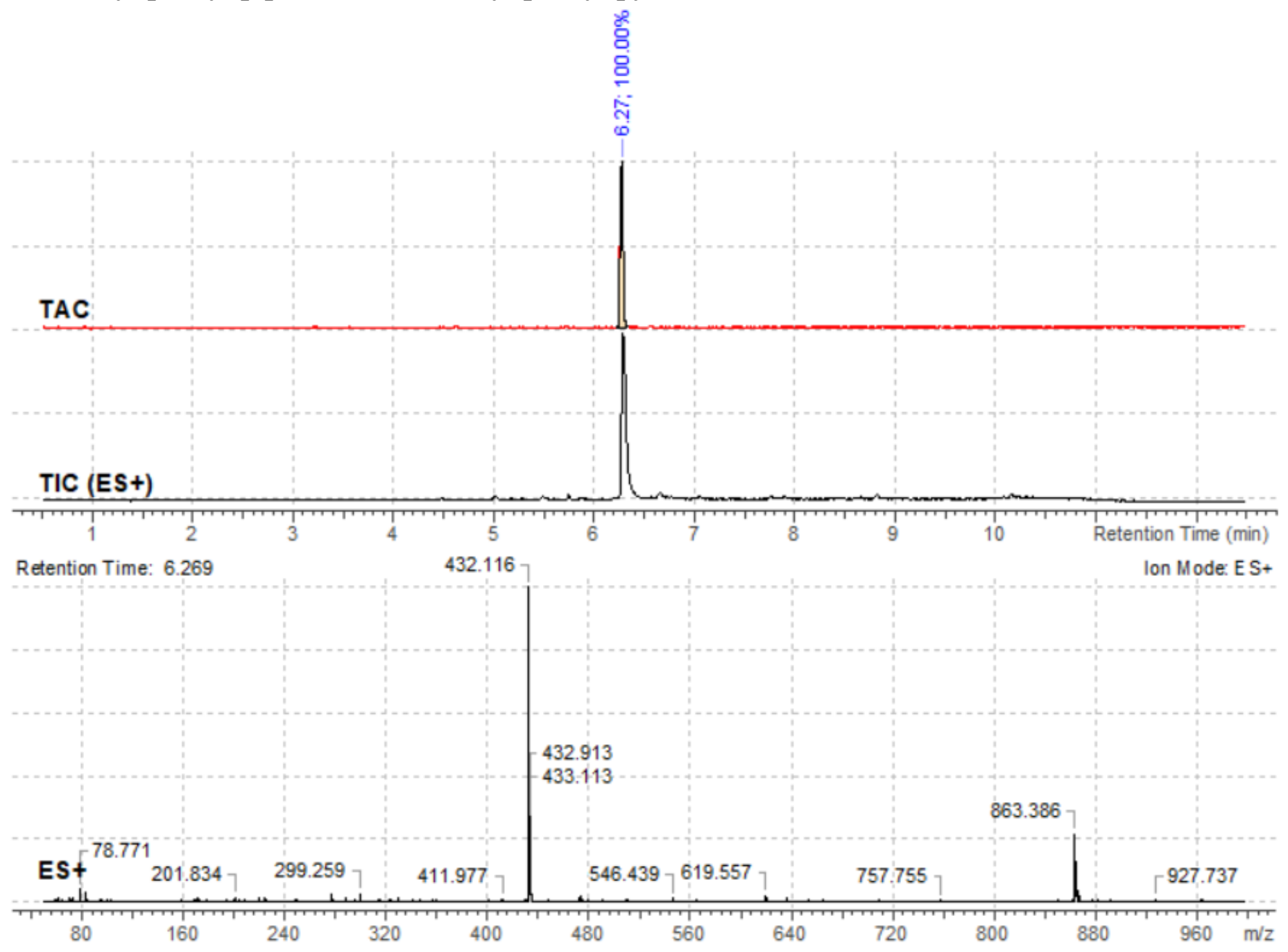


1-(4-(4-(3-(Trifluoromethyl)phenyl)piperazine-1-carbonyl)phenyl)pyrrolidine-2,5-dione (53).

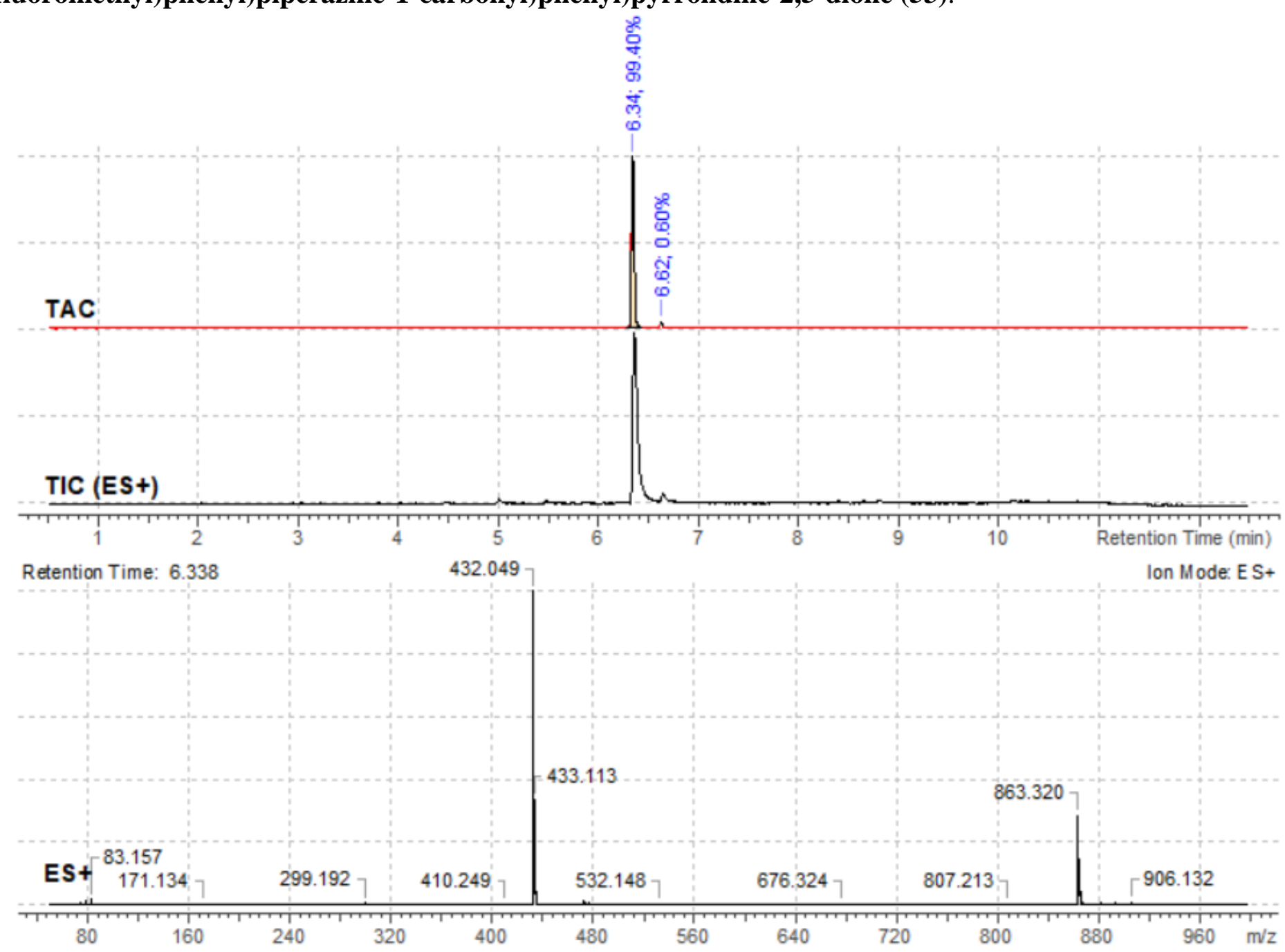


${ }^{1} \mathrm{H}$ NMR, ${ }^{13} \mathrm{C}$ NMR, ${ }^{19} \mathrm{~F}$ NMR spectra for selected starting amines, intermediates and final compounds

1-(3-(Tert-butyl)phenyl)piperazine (A14) - ${ }^{1} \mathrm{H}$ NMR

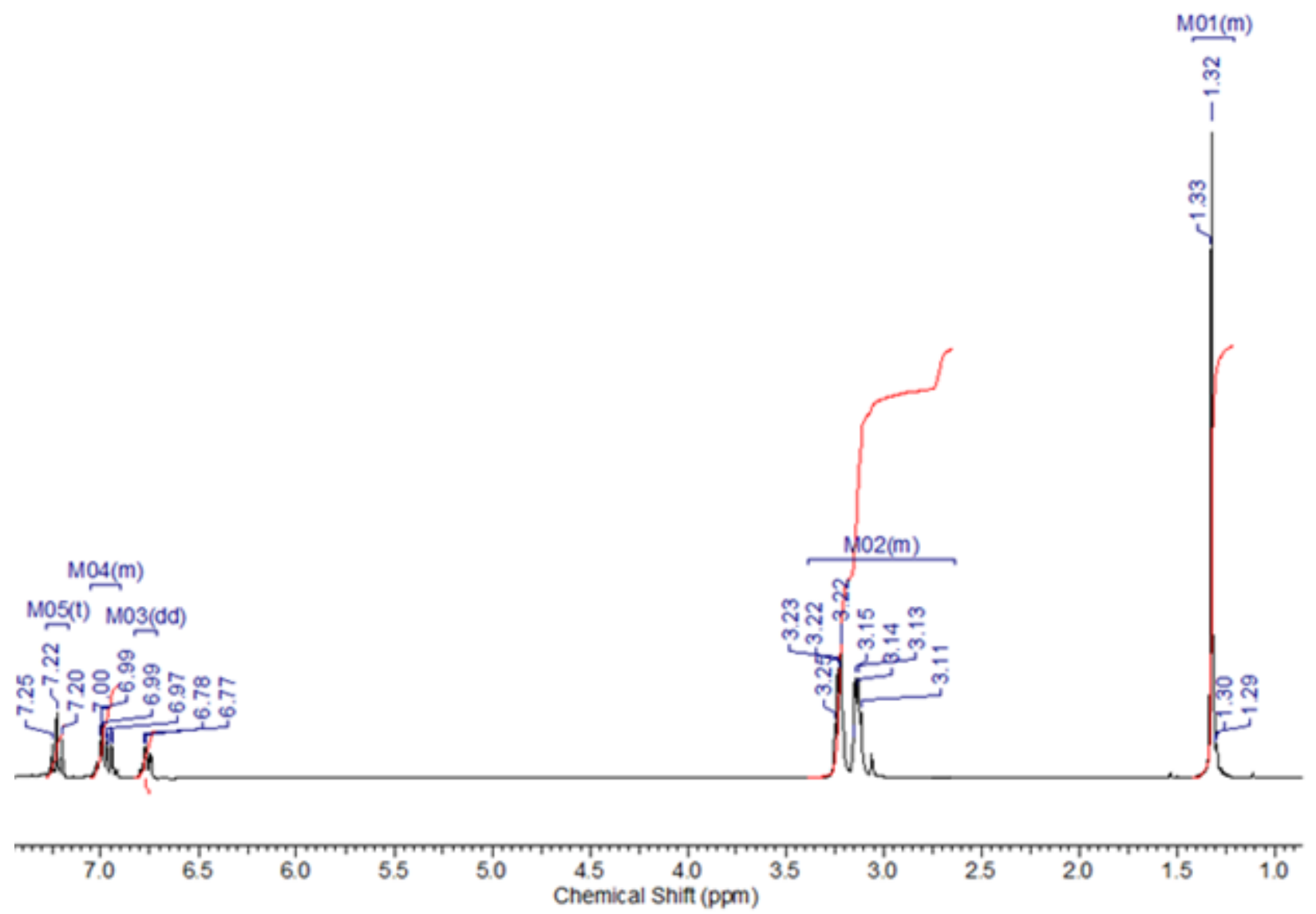


2-(2,5-dioxopyrrolidin-1-yl)-2-phenylacetic acid (6) - ${ }^{1} \mathrm{H}$ NMR

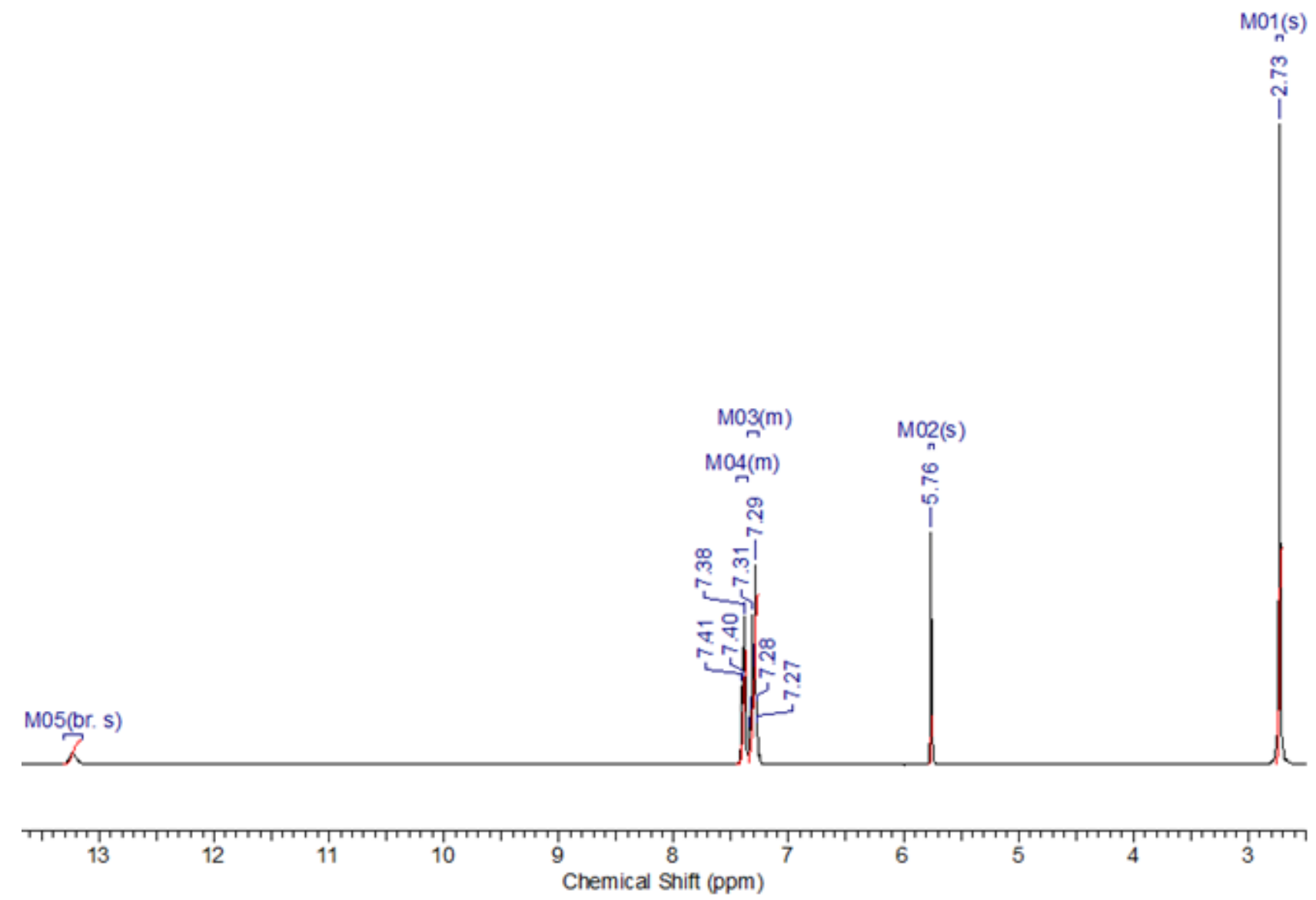


1-(2-Oxo-1-phenyl-2-(4-phenylpiperazin-1-yl)ethyl)pyrrolidine-2,5-dione (11) - ${ }^{1} \mathrm{H}$ NMR

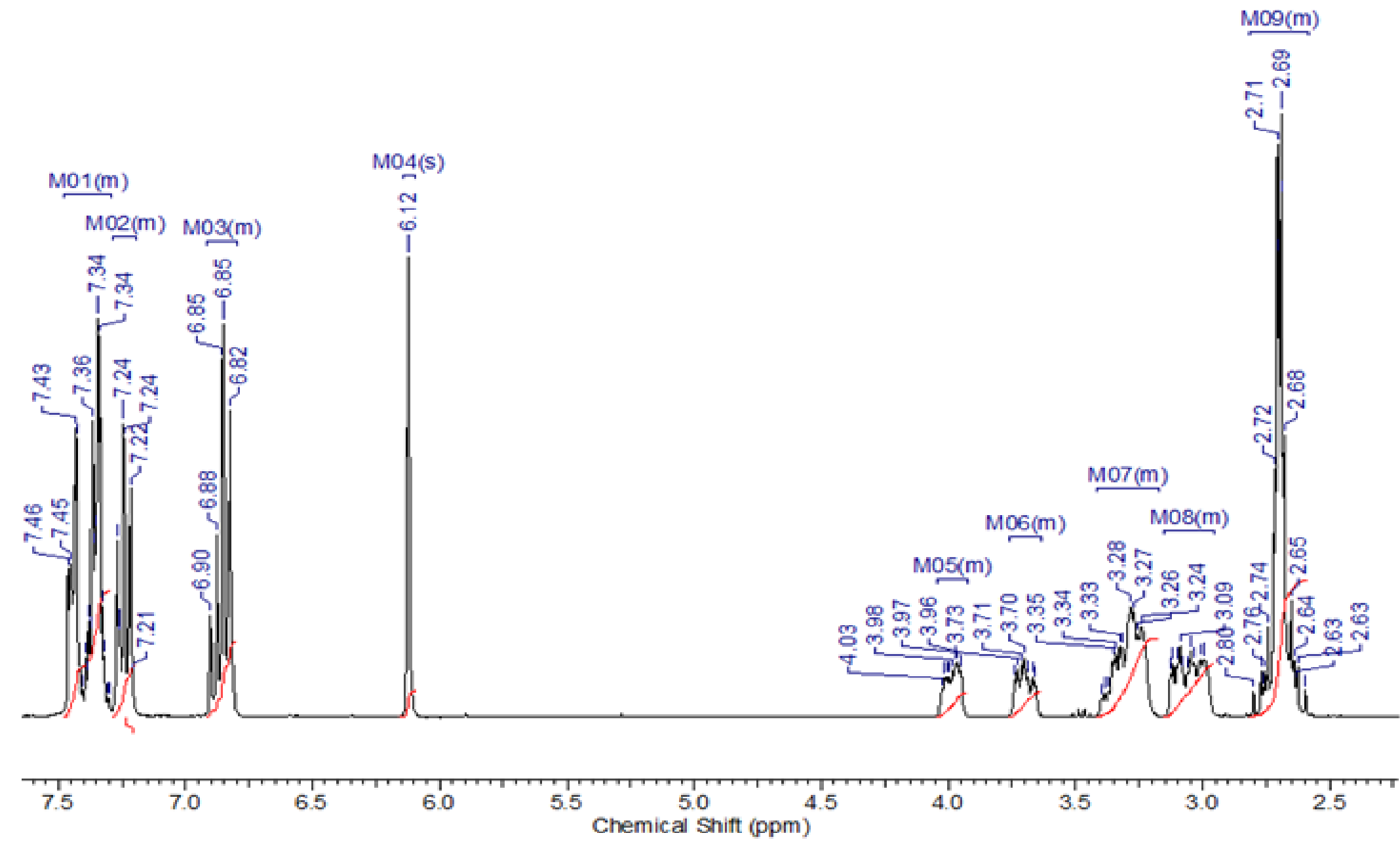


1-(2-Oxo-1-phenyl-2-(4-phenylpiperazin-1-yl)ethyl)pyrrolidine-2,5-dione (11) - ${ }^{13} \mathrm{C} \mathrm{NMR}$

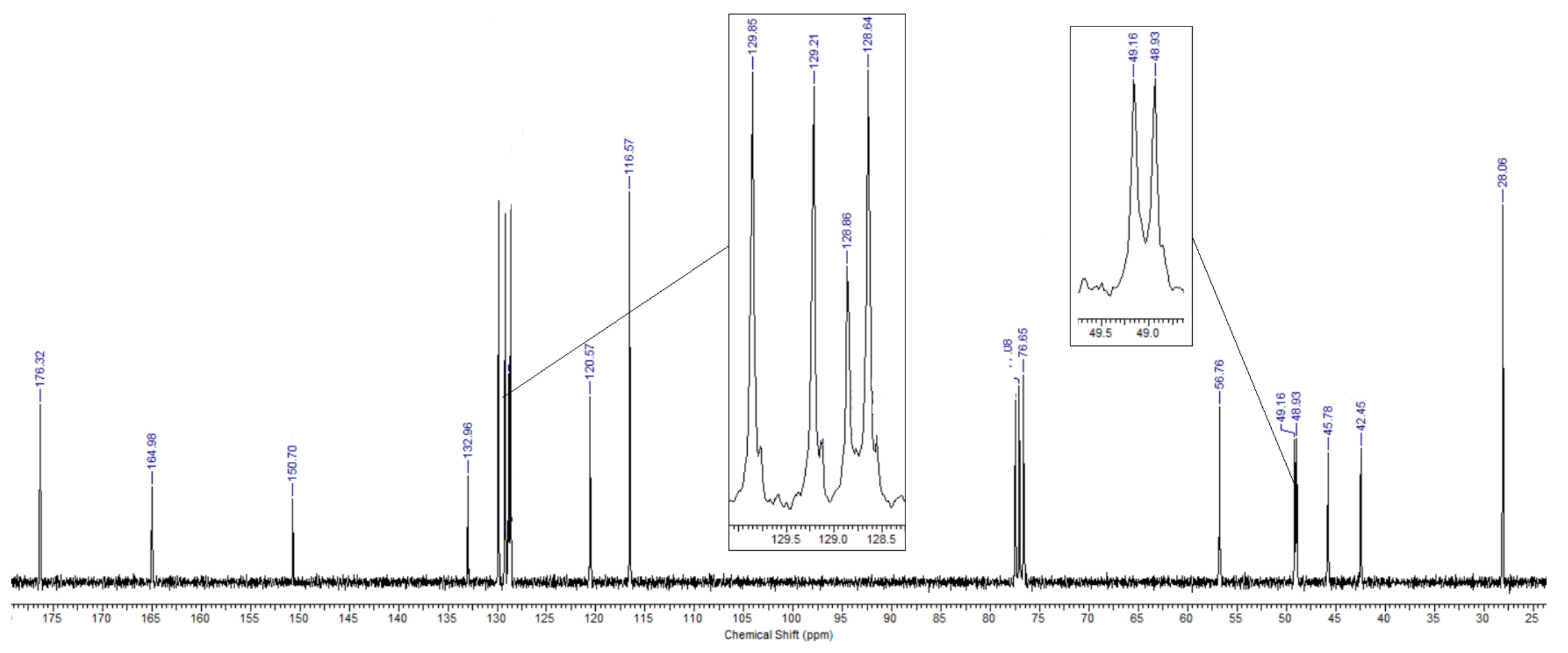


1-(2-(4-(2-Chlorophenyl)piperazin-1-yl)-2-oxo-1-phenylethyl)pyrrolidine-2,5-dione (12) - ${ }^{1} \mathrm{H}$ NMR

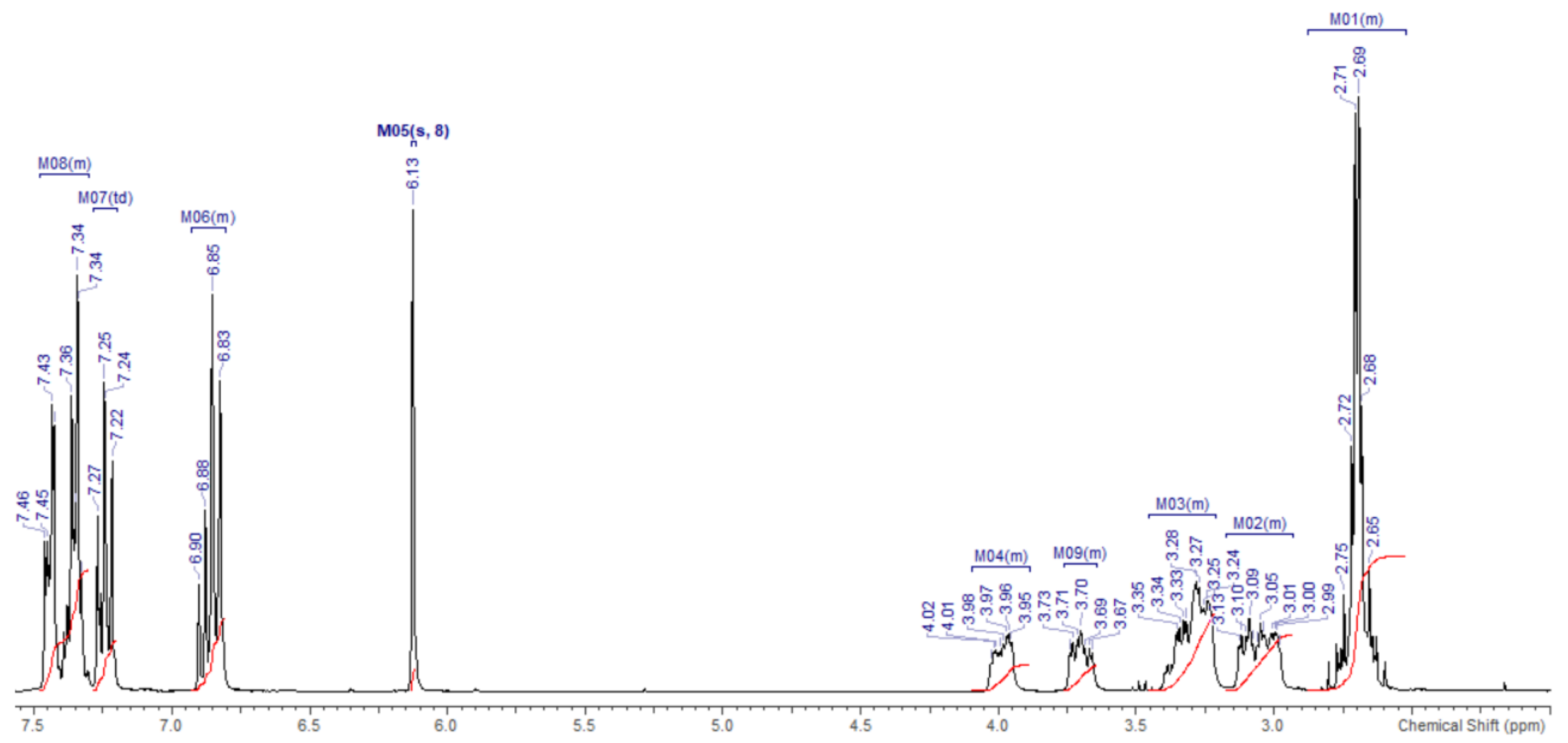


1-(2-(4-(2-Chlorophenyl)piperazin-1-yl)-2-oxo-1-phenylethyl)pyrrolidine-2,5-dione (12) - ${ }^{13} \mathrm{C}$ NMR

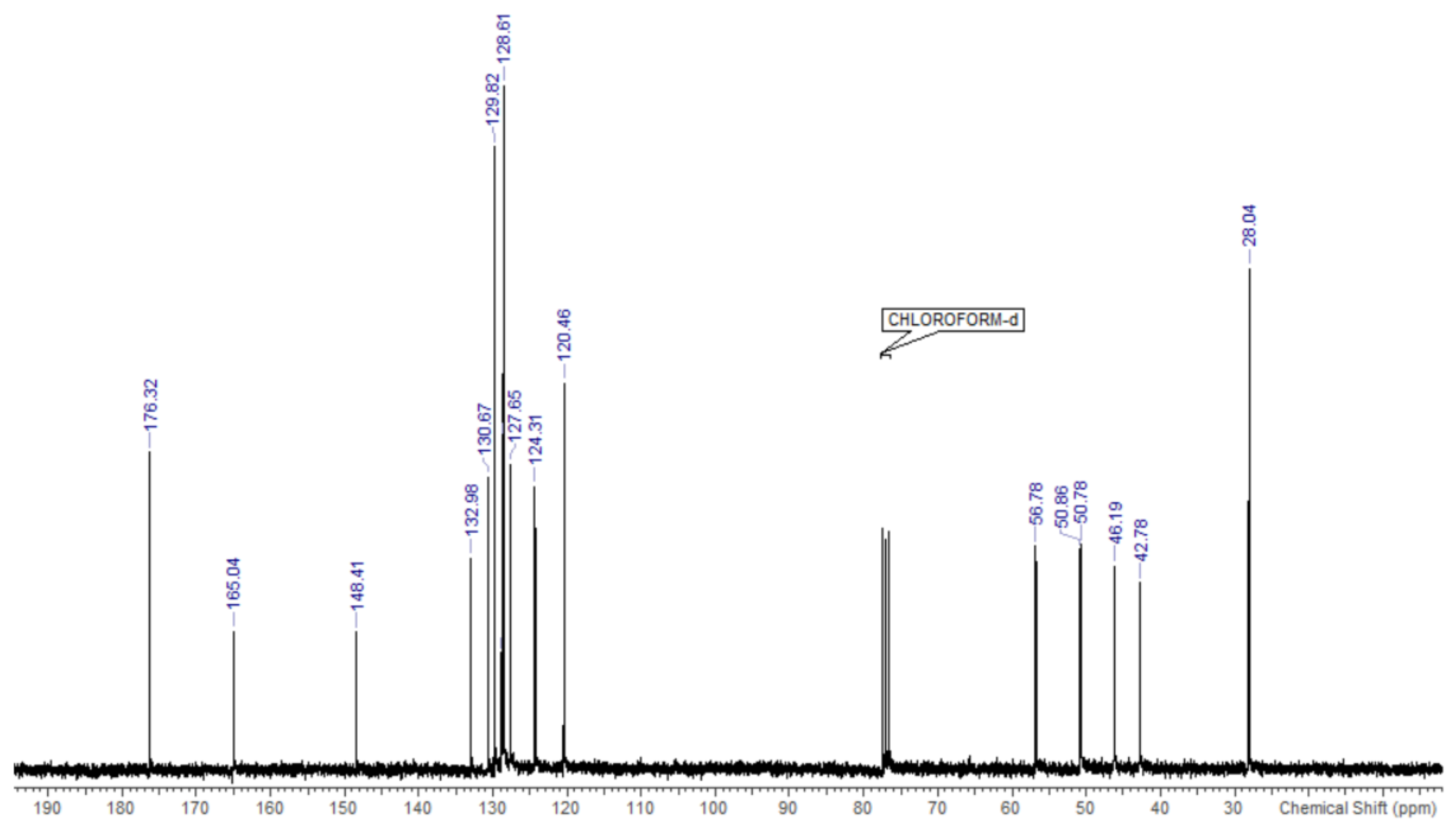


1-(2-(4-(3-Chlorophenyl)piperazin-1-yl)-2-oxo-1-phenylethyl)pyrrolidine-2,5-dione (13) - ${ }^{1} \mathrm{H}$ NMR

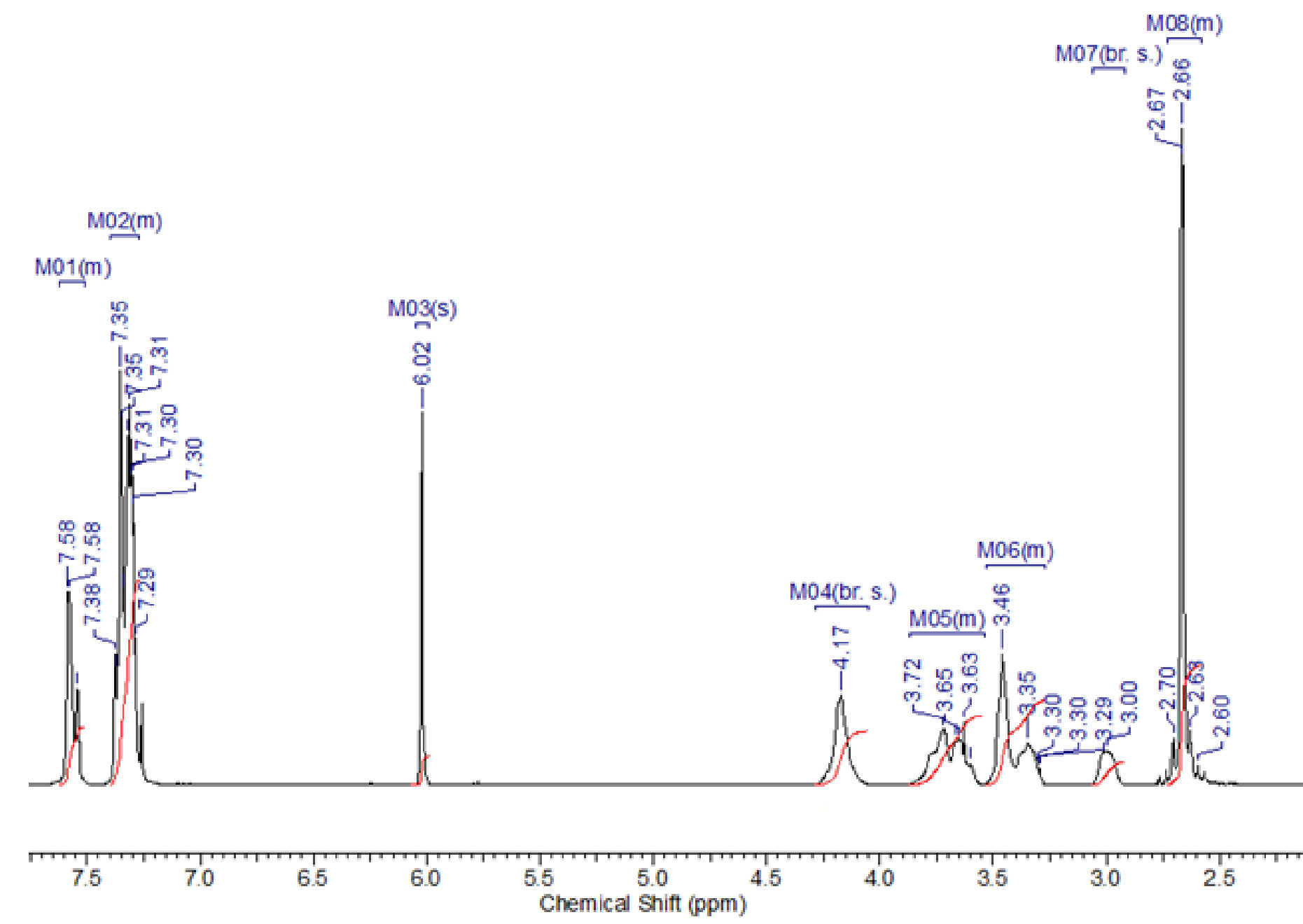


1-(2-(4-(3-Chlorophenyl)piperazin-1-yl)-2-oxo-1-phenylethyl)pyrrolidine-2,5-dione (13) - ${ }^{13} \mathrm{C}$ NMR

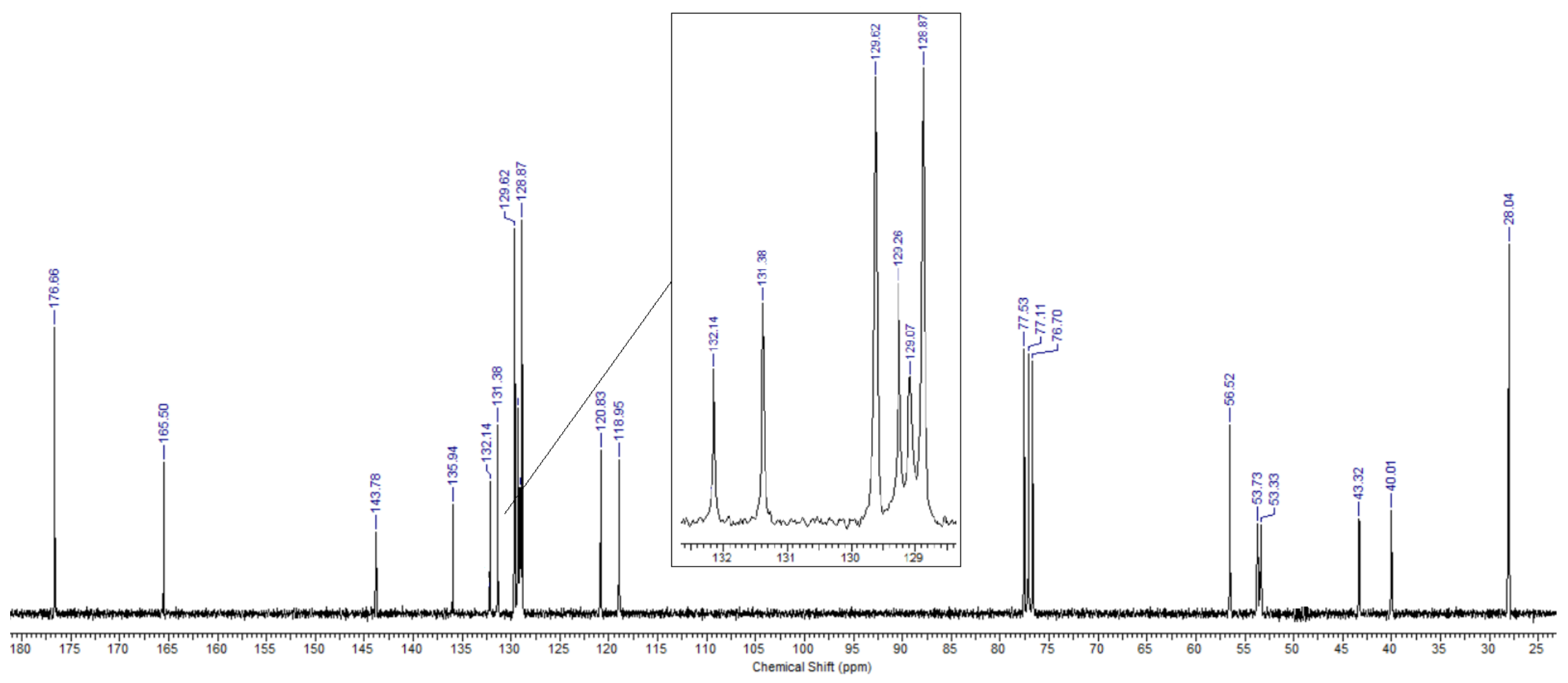


1-(2-(4-(4-Chlorophenyl)piperazin-1-yl)-2-oxo-1-phenylethyl)pyrrolidine-2,5-dione (14) - ${ }^{1} \mathrm{H}$ NMR

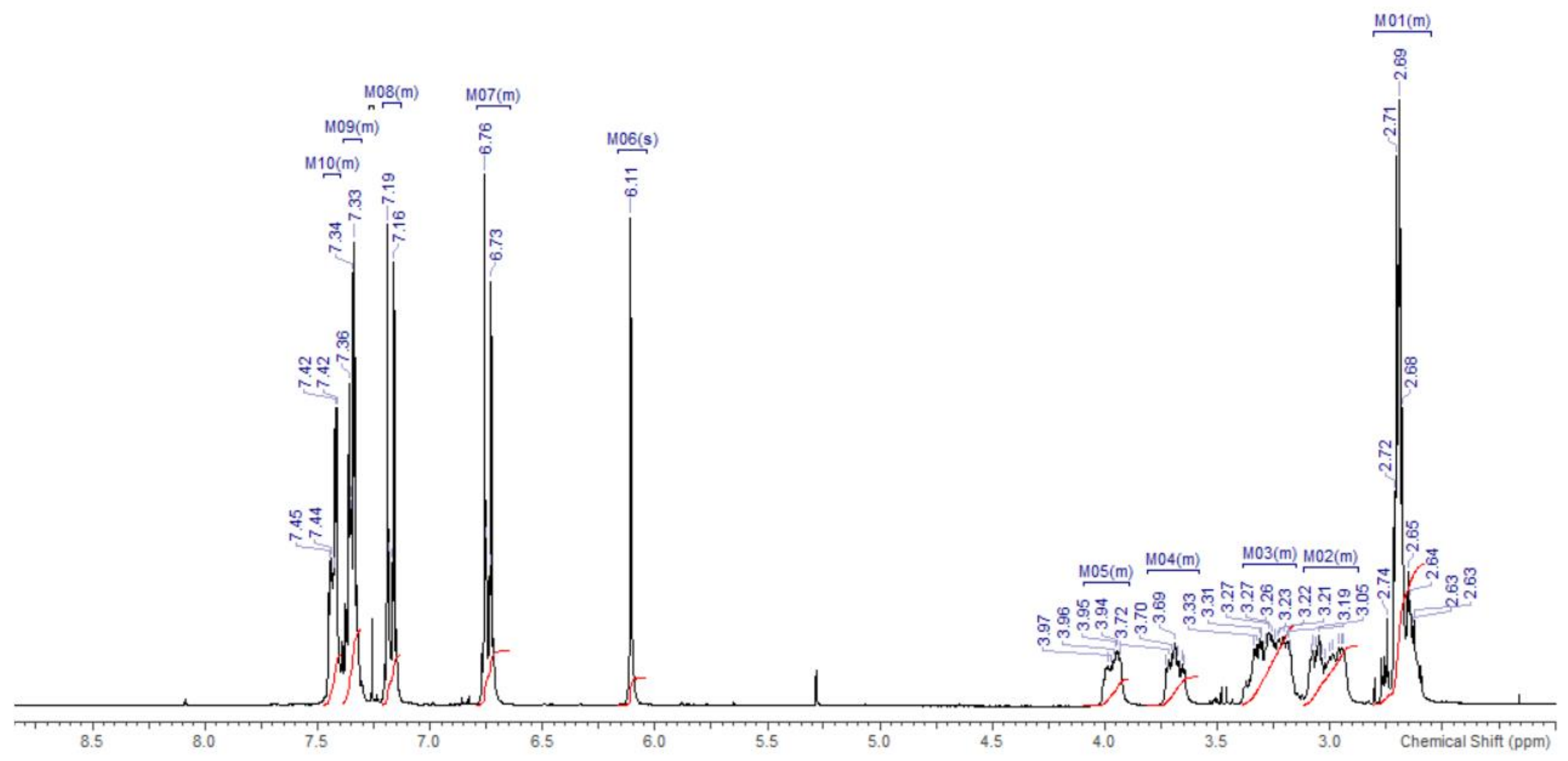


1-(2-(4-(4-Chlorophenyl)piperazin-1-yl)-2-oxo-1-phenylethyl)pyrrolidine-2,5-dione (14) - ${ }^{13} \mathrm{C}$ NMR

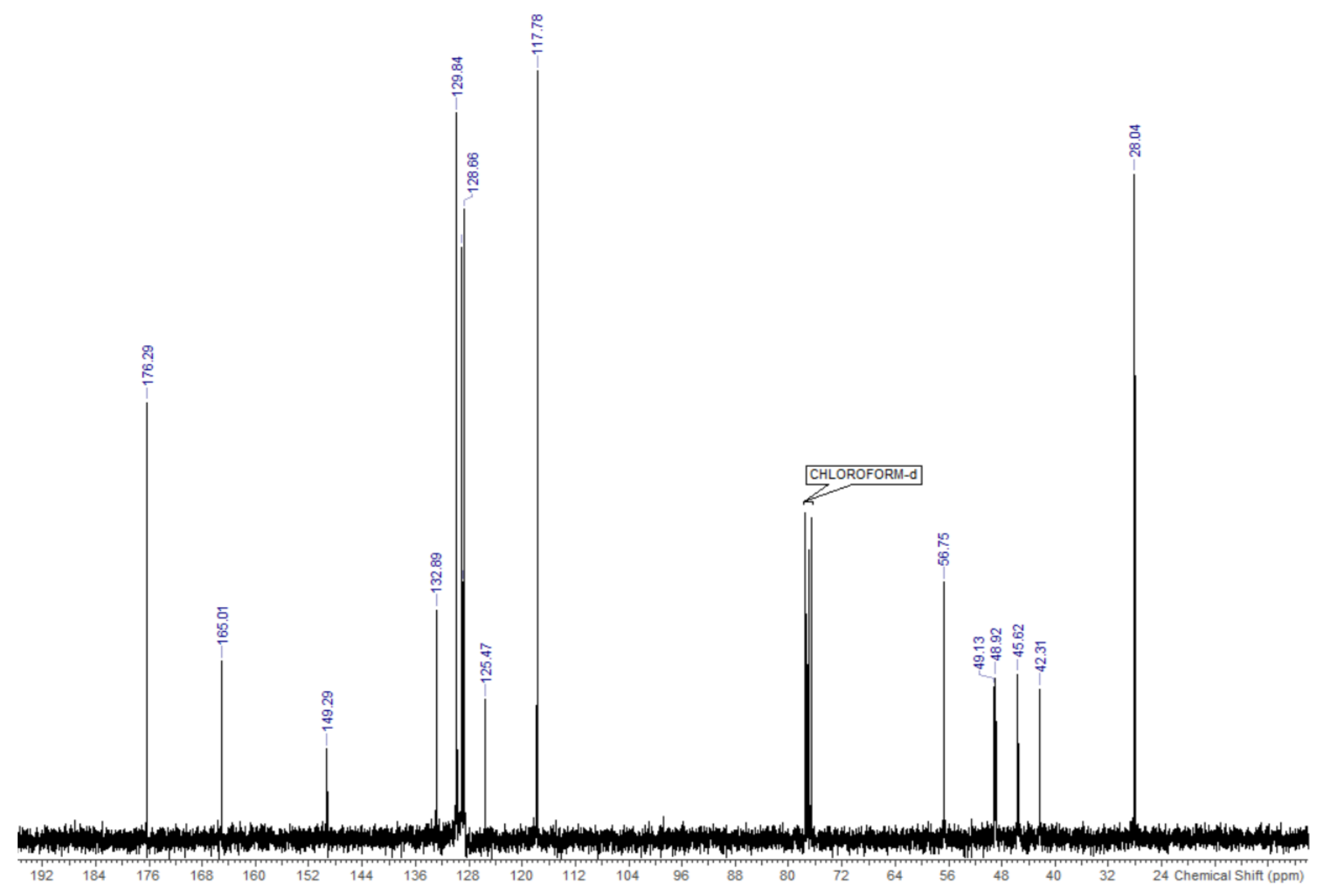


1-(2-(4-(2-Fluorophenyl)piperazin-1-yl)-2-oxo-1-phenylethyl)pyrrolidine-2,5-dione (15) - ${ }^{1} \mathrm{H}$ NMR

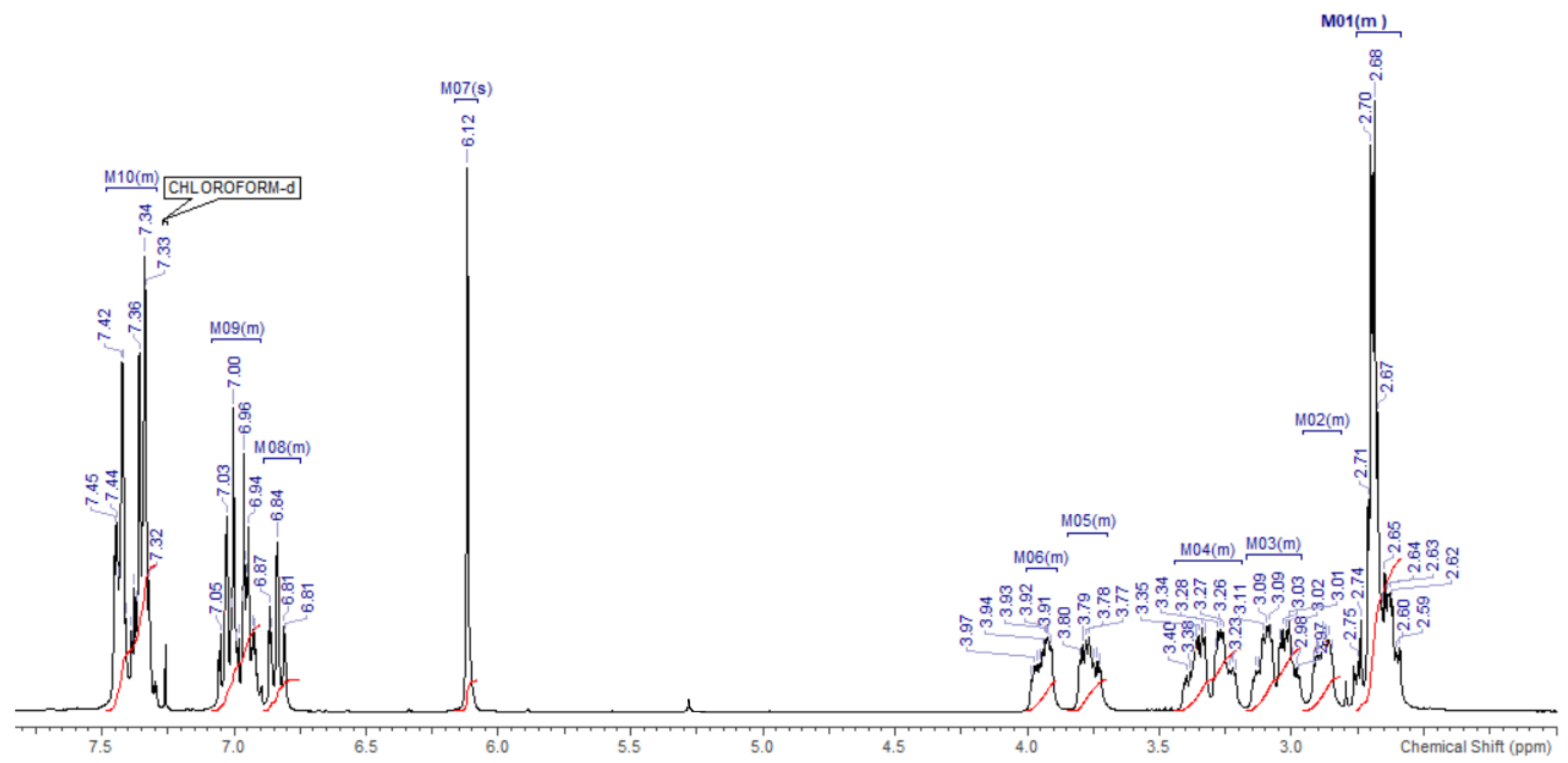


1-(2-(4-(2-Fluorophenyl)piperazin-1-yl)-2-oxo-1-phenylethyl)pyrrolidine-2,5-dione (15) - ${ }^{13} \mathrm{C}$ NMR

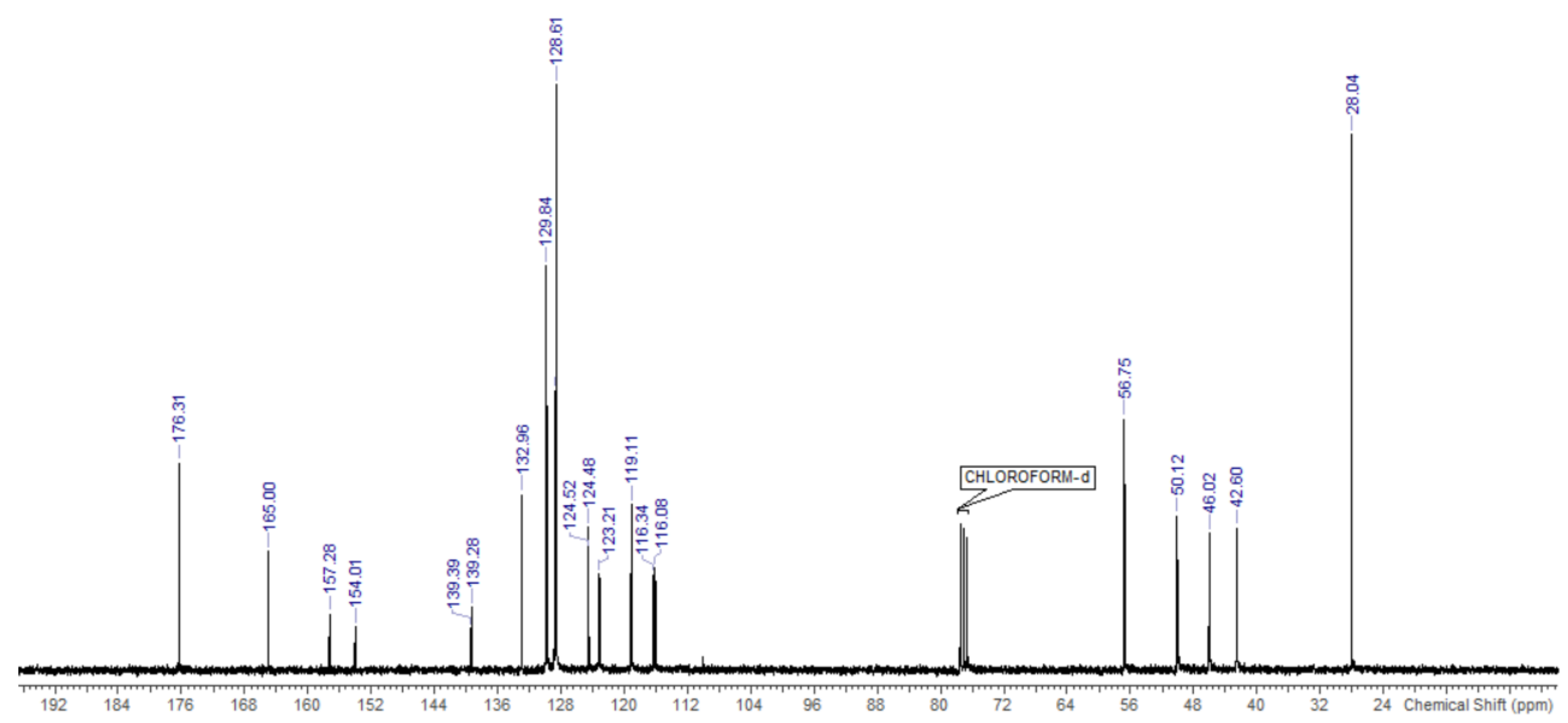


1-(2-(4-(3-Fluorophenyl)piperazin-1-yl)-2-oxo-1-phenylethyl)pyrrolidine-2,5-dione (16) - ${ }^{1} \mathrm{H}$ NMR

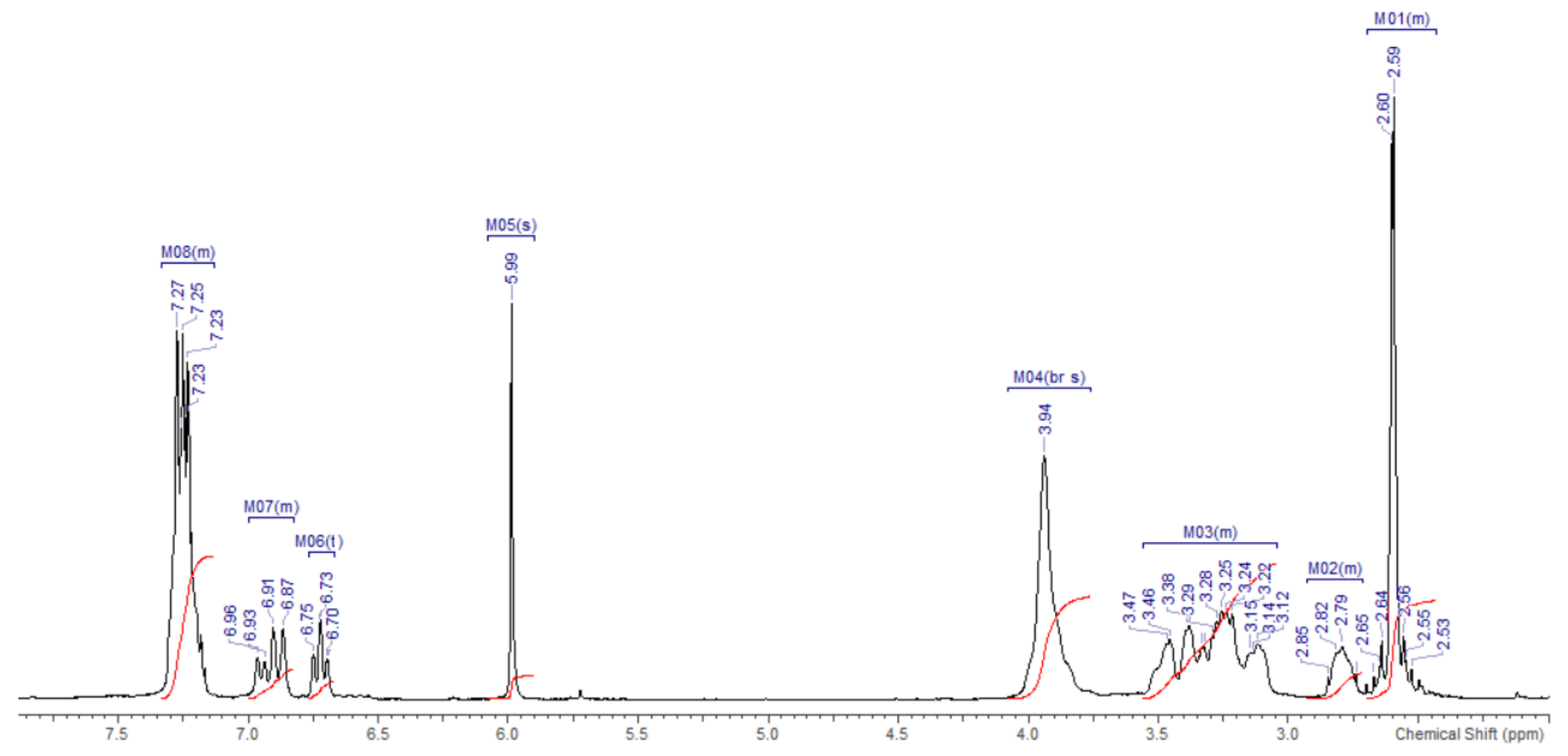


1-(2-(4-(3-Fluorophenyl)piperazin-1-yl)-2-oxo-1-phenylethyl)pyrrolidine-2,5-dione (16) - ${ }^{13} \mathrm{C}$ NMR

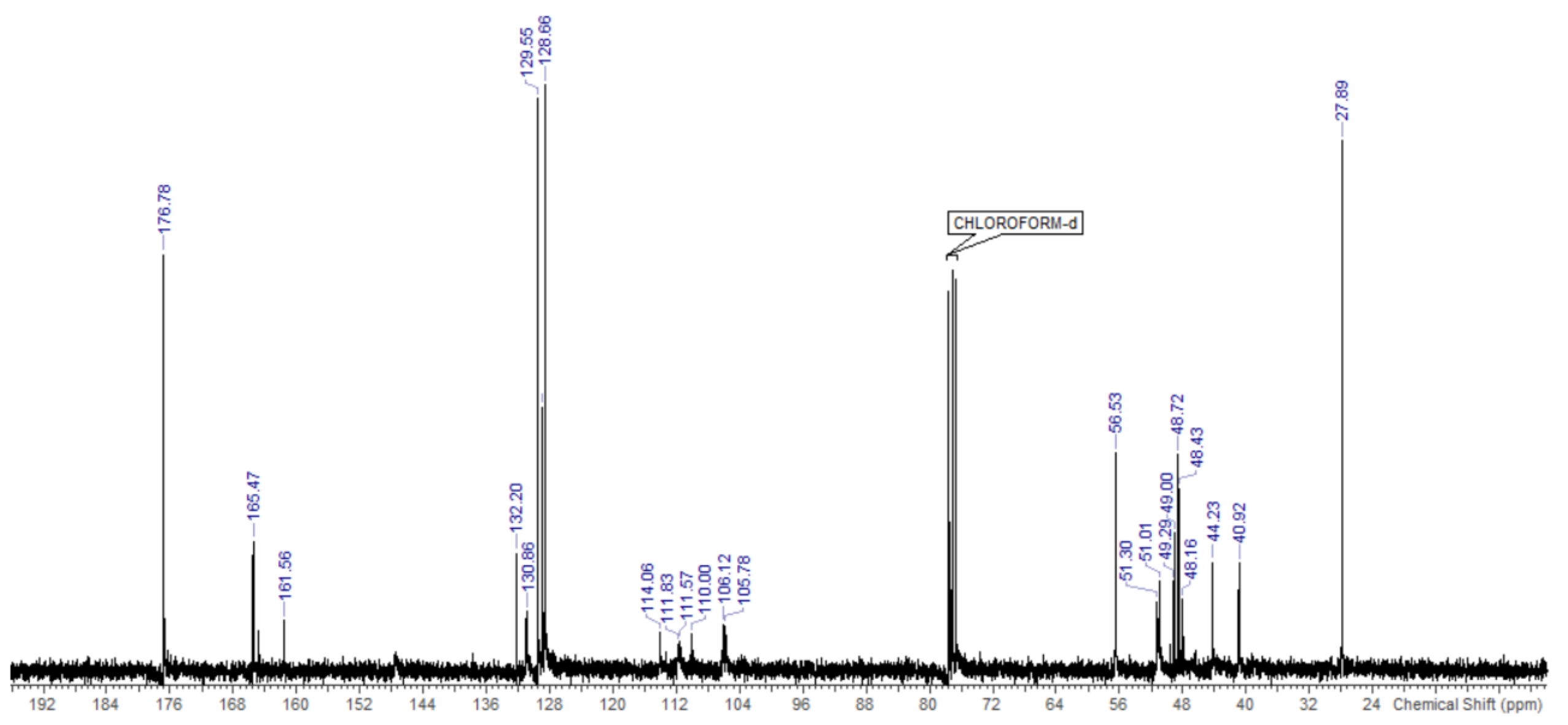


1-(2-(4-(4-Fluorophenyl)piperazin-1-yl)-2-oxo-1-phenylethyl)pyrrolidine-2,5-dione (17) - ${ }^{1} \mathrm{H}$ NMR

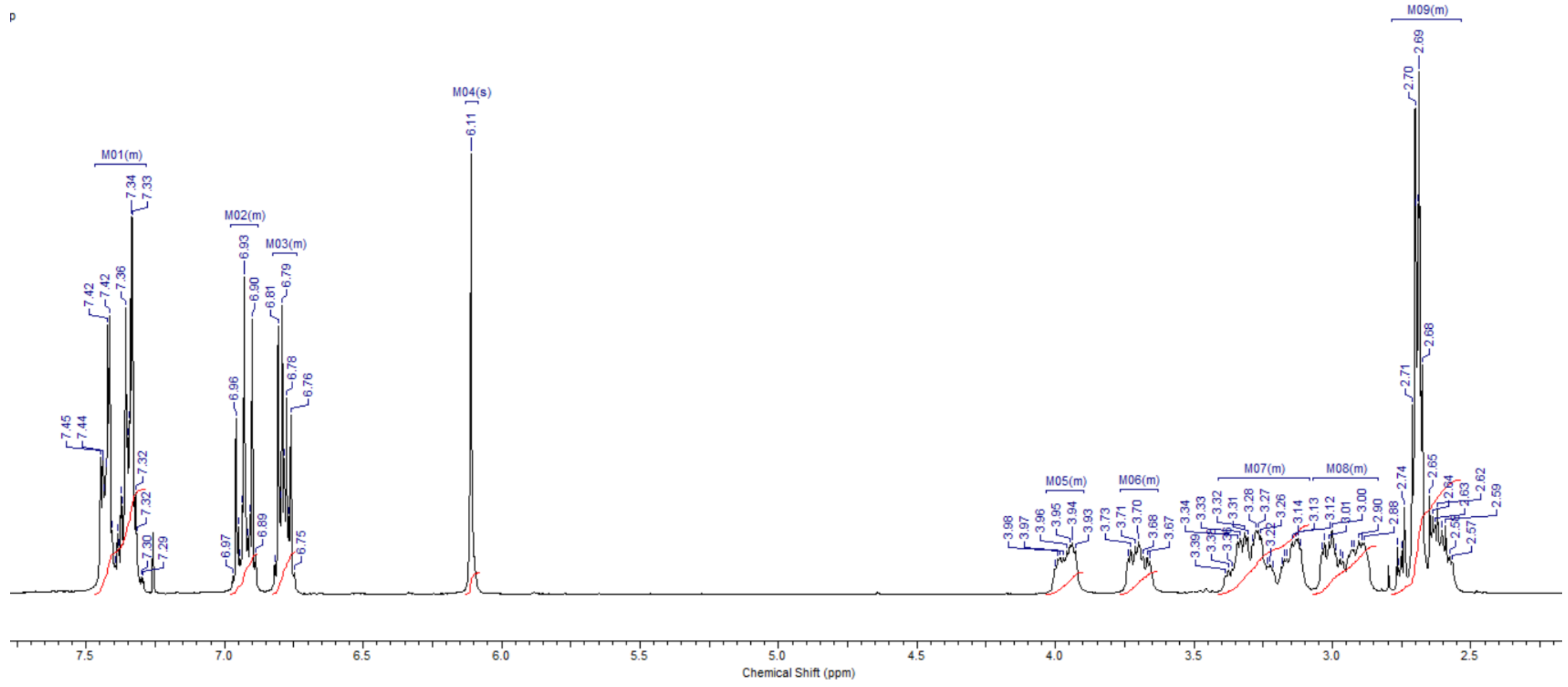


1-(2-(4-(4-Fluorophenyl)piperazin-1-yl)-2-oxo-1-phenylethyl)pyrrolidine-2,5-dione (17) - ${ }^{13} \mathrm{C}$ NMR

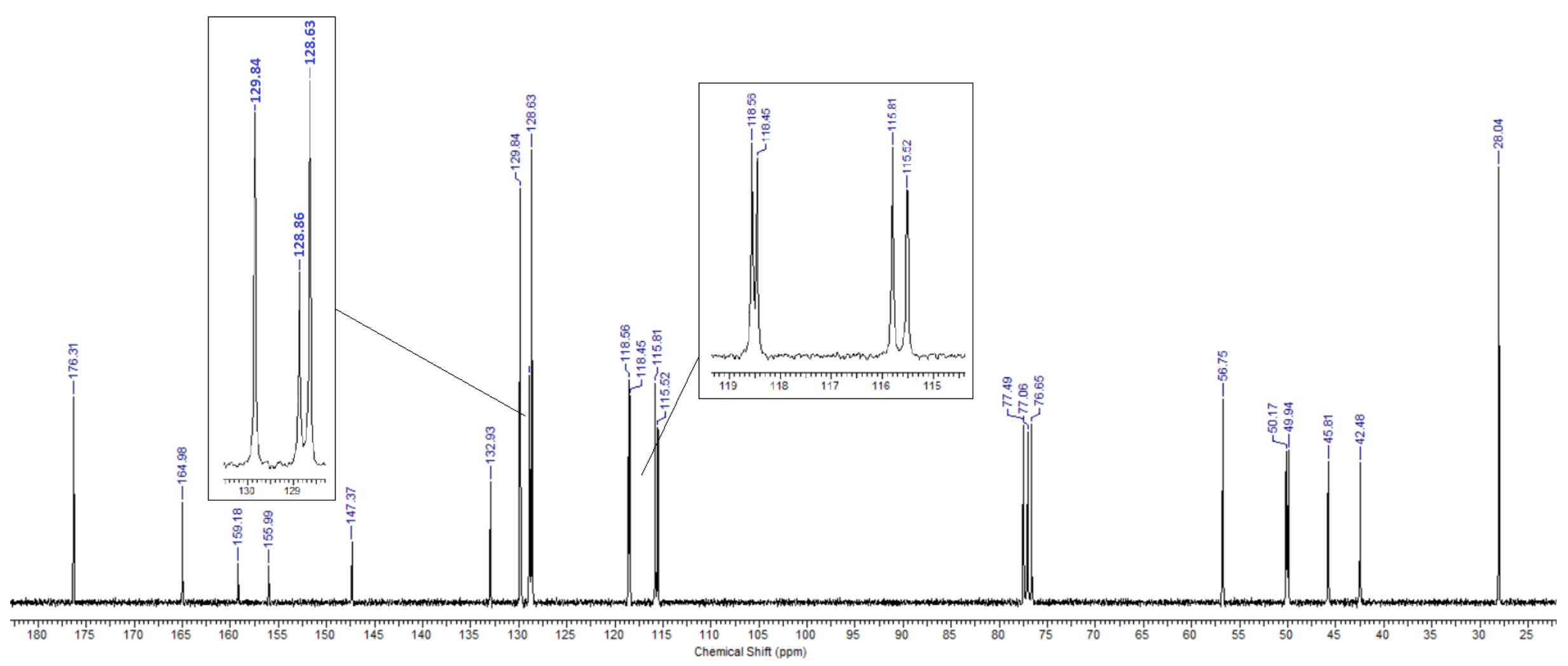


1-(2-(4-(4-Fluorophenyl)piperazin-1-yl)-2-oxo-1-phenylethyl)pyrrolidine-2,5-dione (17) - ${ }^{18}$ F NMR

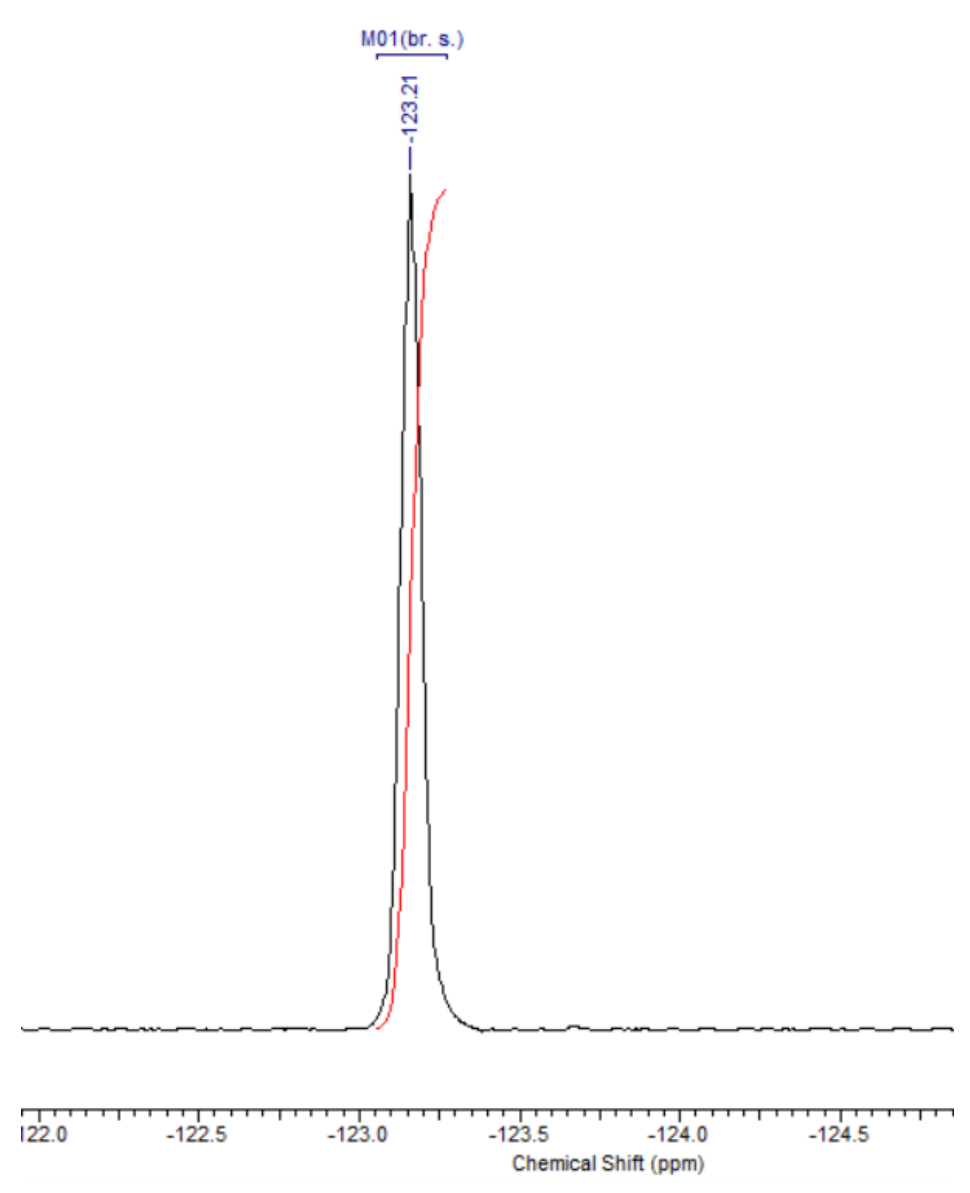


1-(2-Oxo-1-phenyl-2-(4-(o-tolyl)piperazin-1-yl)ethyl)pyrrolidine-2,5-dione (18) - ${ }^{1} \mathrm{H}$ NMR

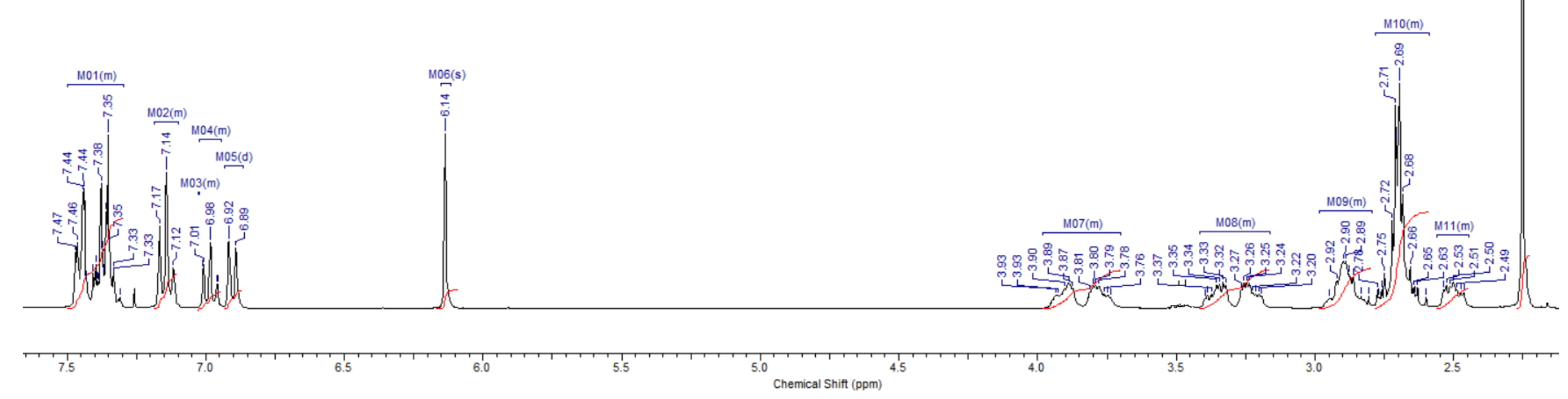


1-(2-Oxo-1-phenyl-2-(4-(o-tolyl)piperazin-1-yl)ethyl)pyrrolidine-2,5-dione (18) - ${ }^{13} \mathrm{C}$ NMR

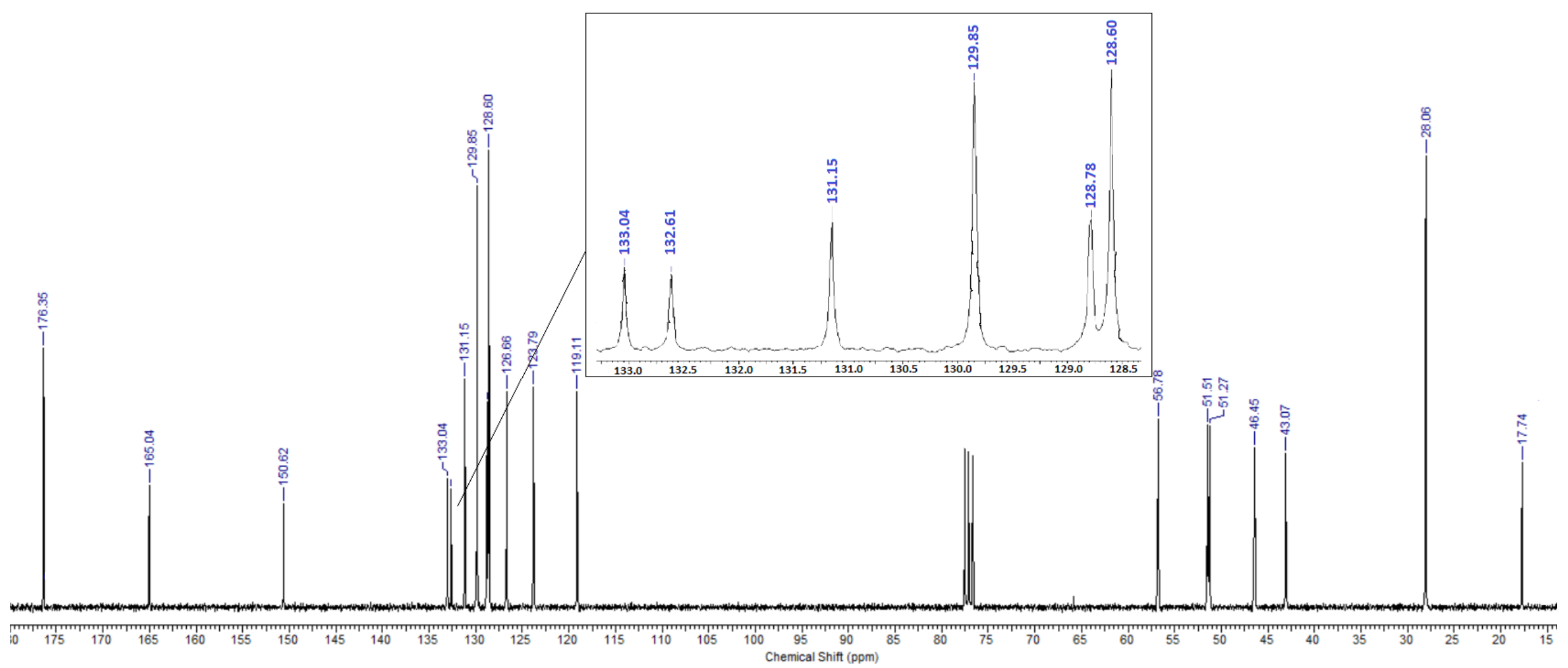


1-(2-Oxo-1-phenyl-2-(4-(m-tolyl)piperazin-1-yl)ethyl)pyrrolidine-2,5-dione (19) - ${ }^{1} \mathrm{H}$ NMR

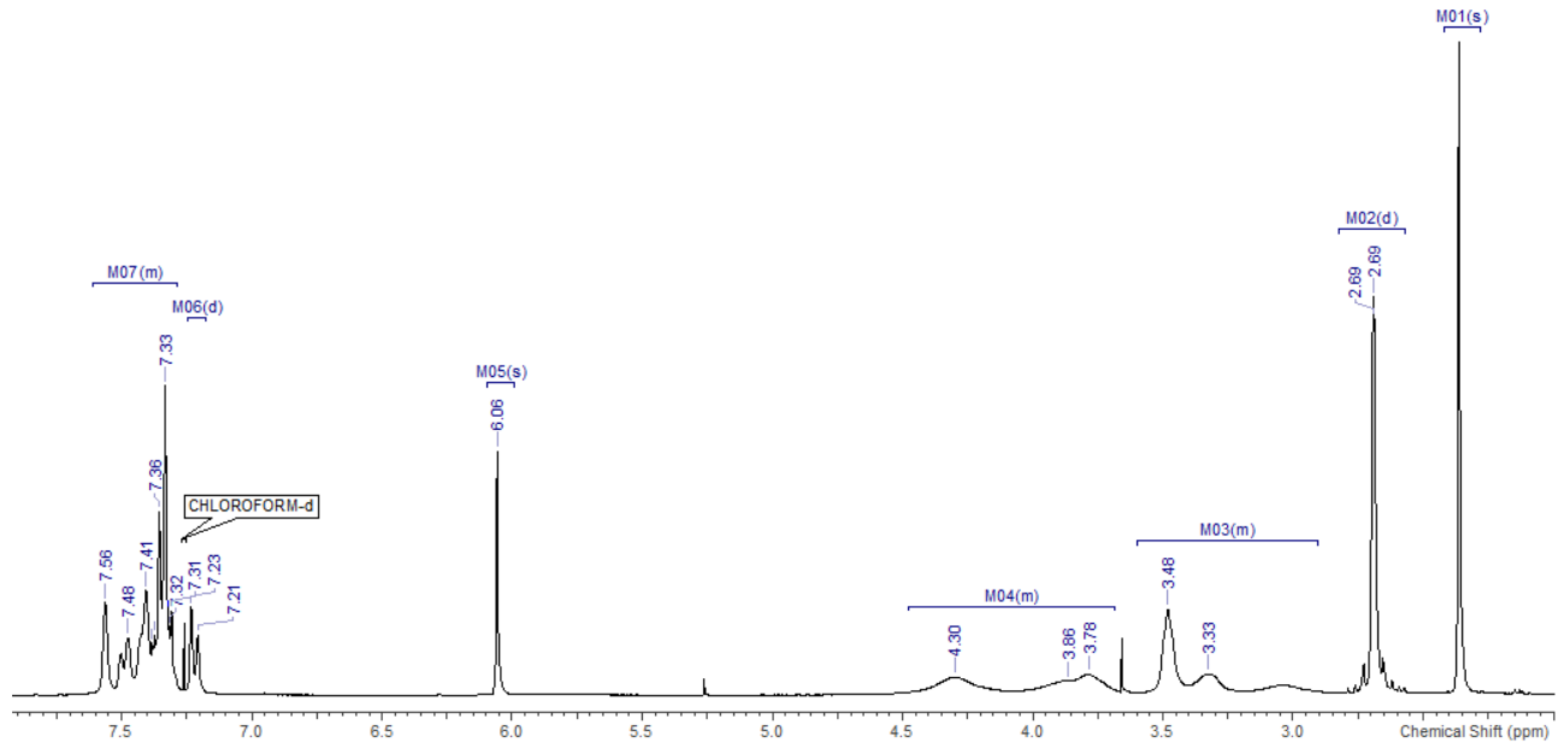


1-(2-Oxo-1-phenyl-2-(4-(m-tolyl)piperazin-1-yl)ethyl)pyrrolidine-2,5-dione (19) - ${ }^{13} \mathrm{C} \mathrm{NMR}$

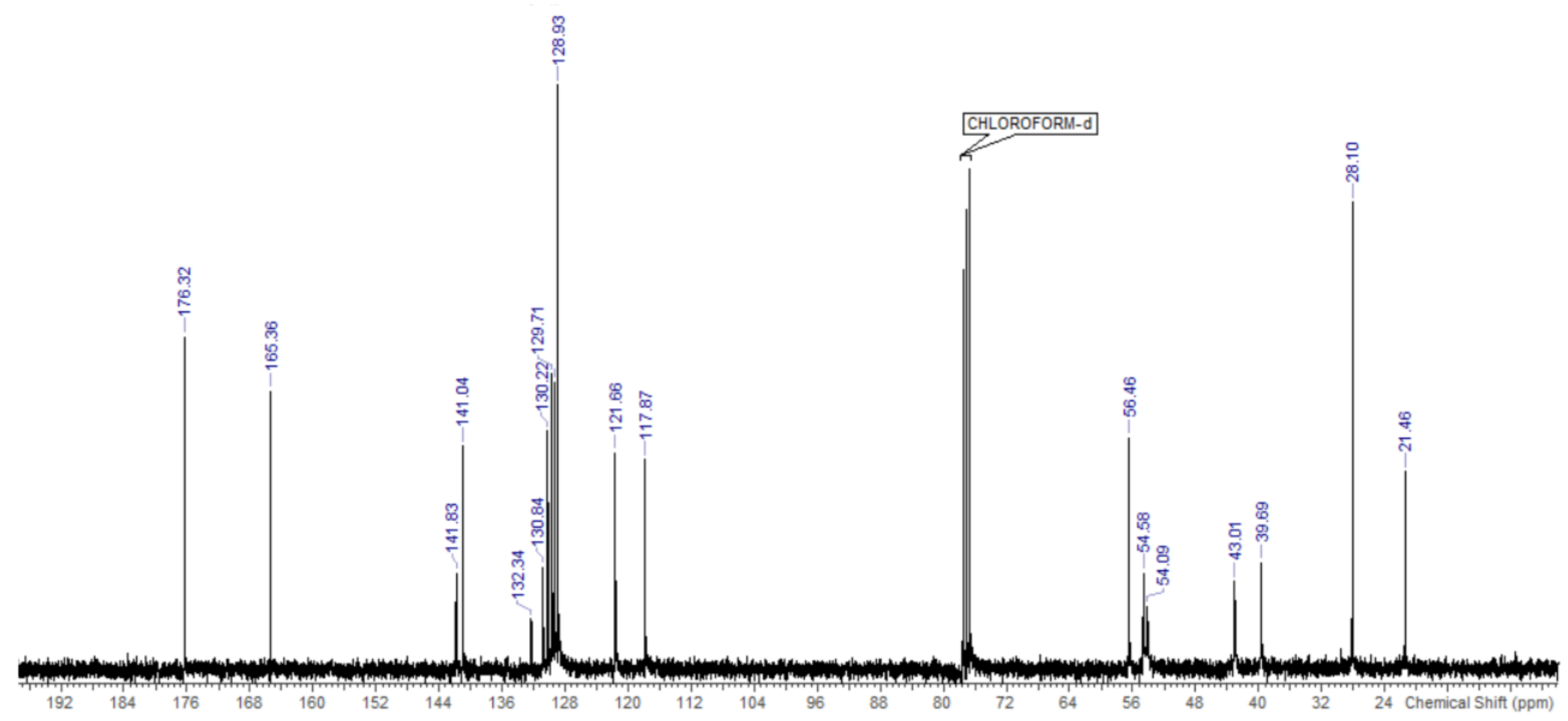


1-(2-Oxo-1-phenyl-2-(4-(p-tolyl)piperazin-1-yl)ethyl)pyrrolidine-2,5-dione (20) - ${ }^{1} \mathrm{H}$ NMR

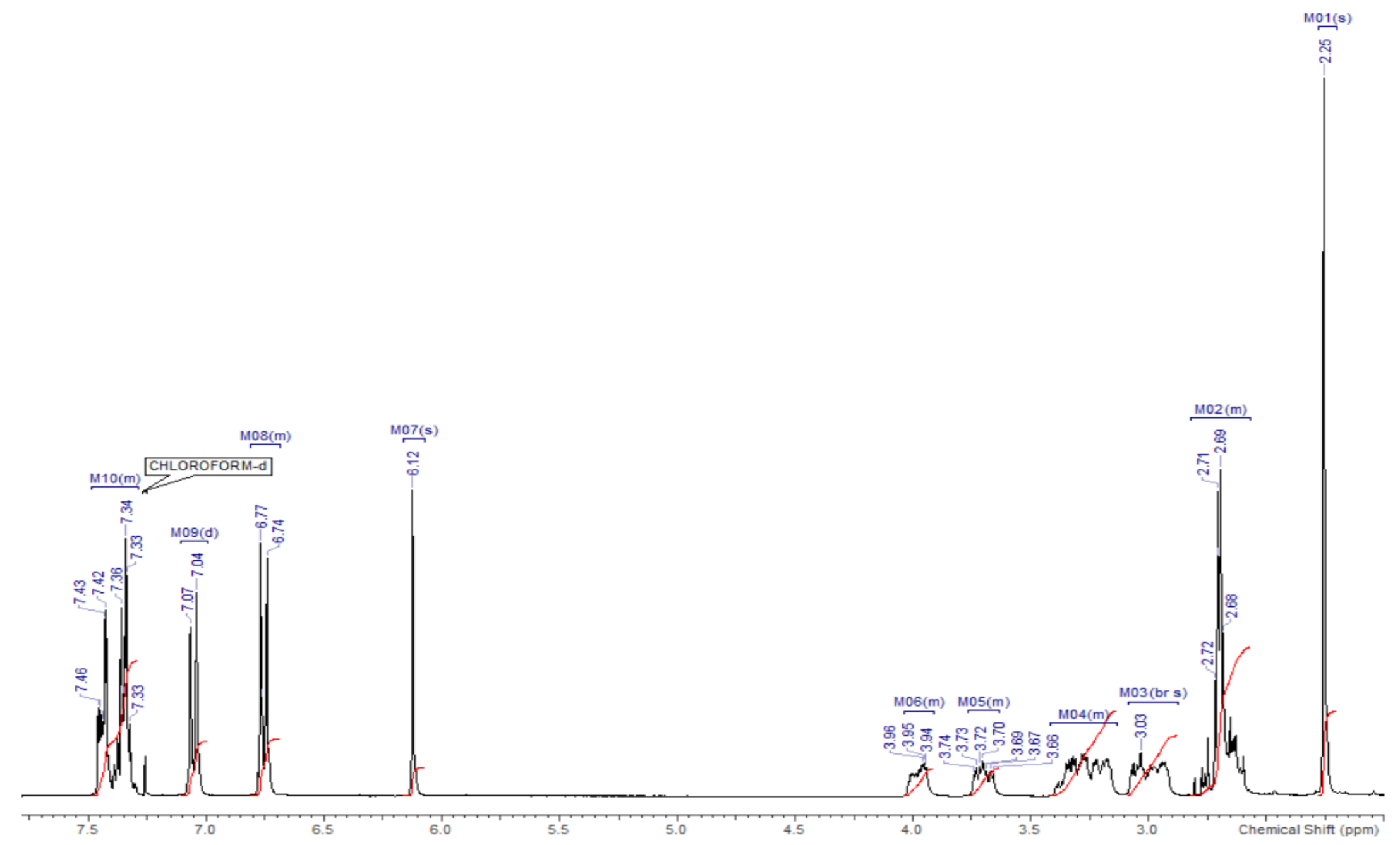


1-(2-Oxo-1-phenyl-2-(4-(p-tolyl)piperazin-1-yl)ethyl)pyrrolidine-2,5-dione (20) - ${ }^{13} \mathrm{C}$ NMR

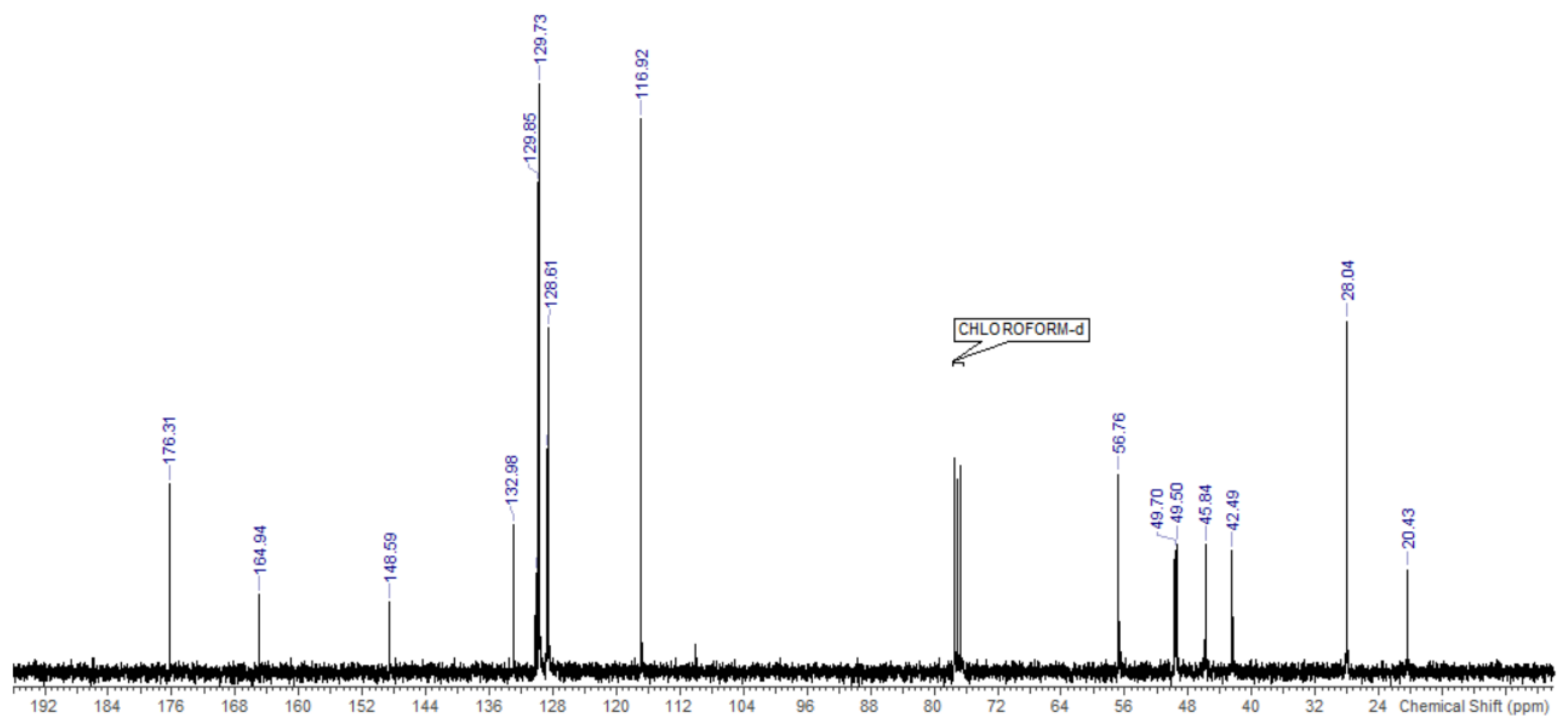


1-(2-Oxo-1-phenyl-2-(4-(2-(trifluoromethyl)phenyl)piperazin-1-yl)ethyl)pyrrolidine-2,5-dione (21) - ${ }^{1} \mathrm{H}$ NMR

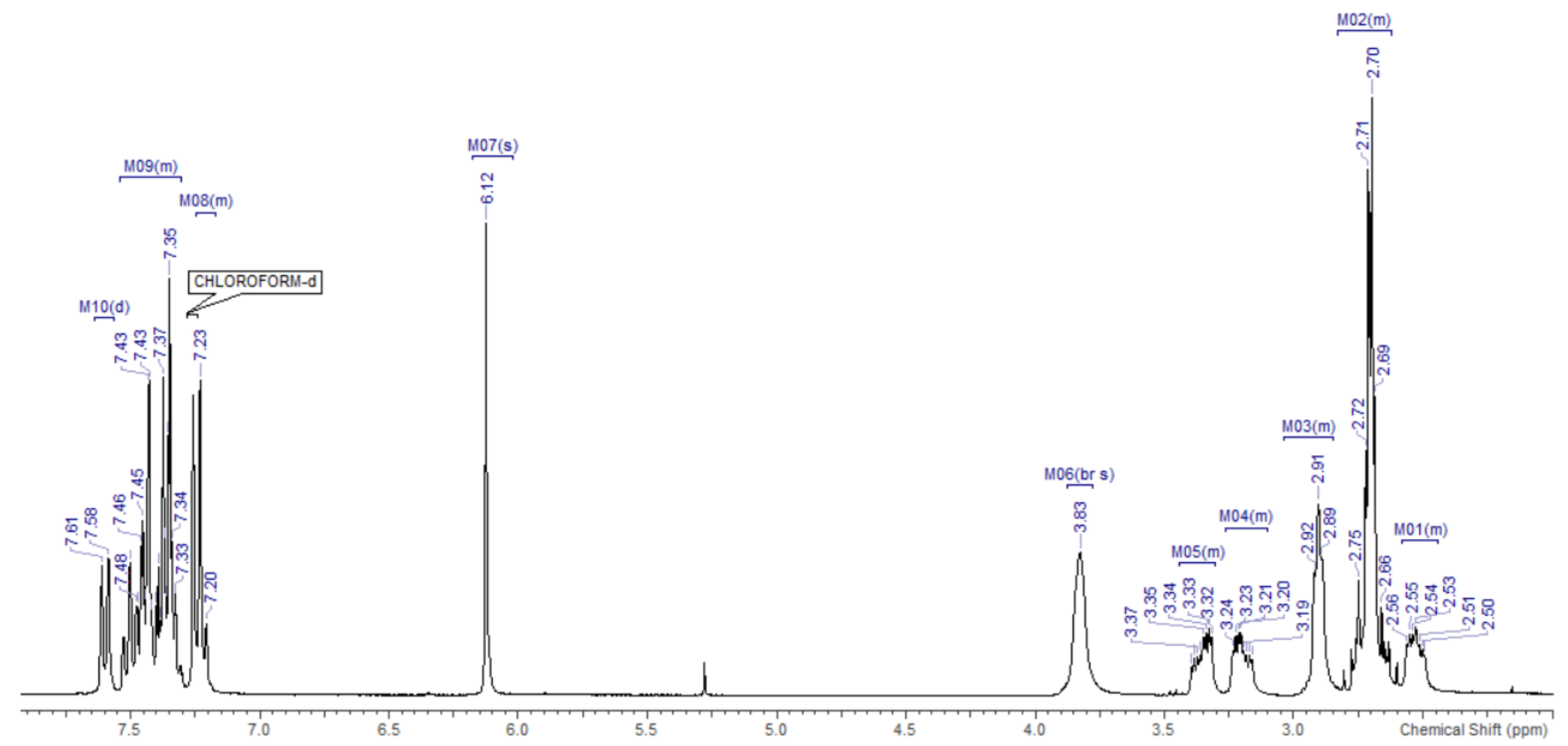


1-(2-Oxo-1-phenyl-2-(4-(2-(trifluoromethyl)phenyl)piperazin-1-yl)ethyl)pyrrolidine-2,5-dione (21) - ${ }^{13} \mathrm{C}$ NMR
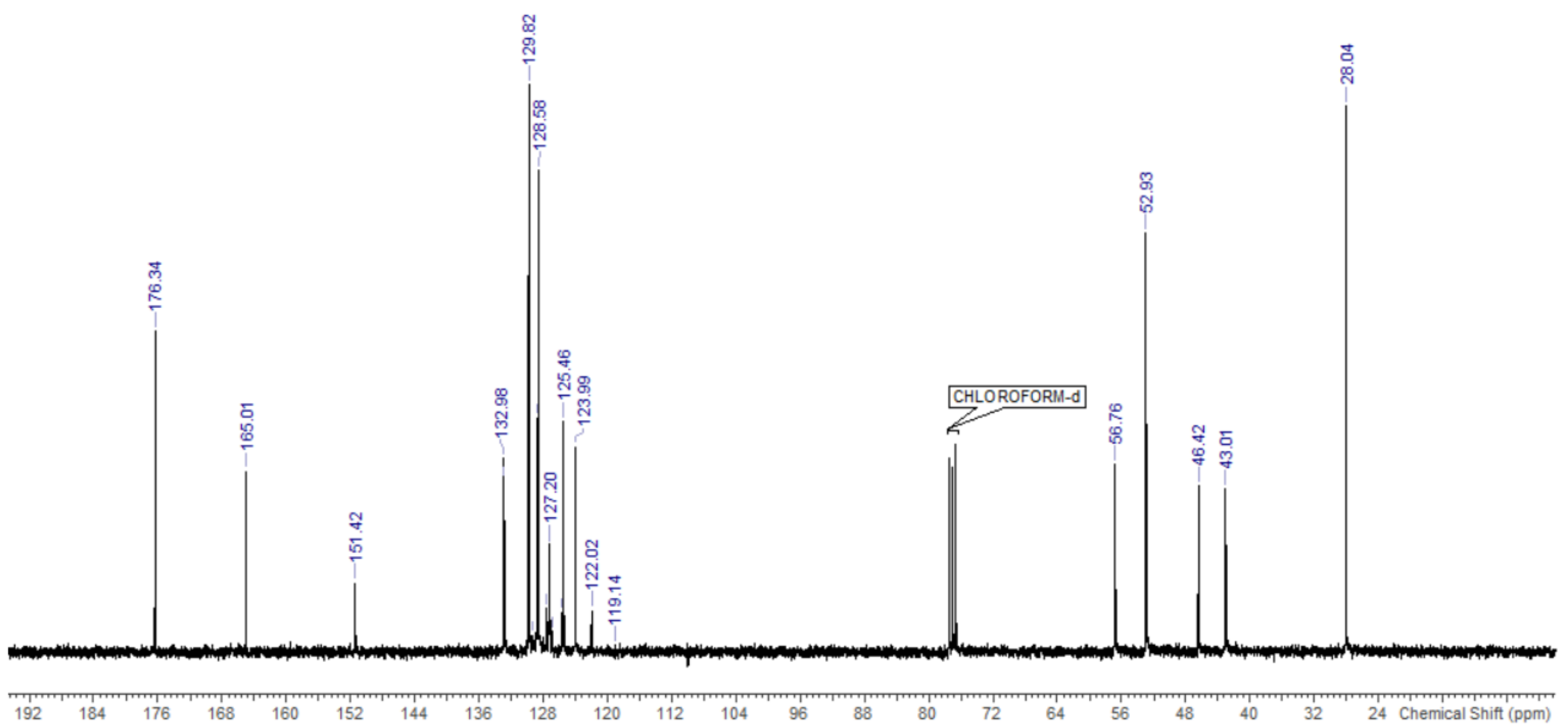
1-(2-Oxo-1-phenyl-2-(4-(3-(trifluoromethyl)phenyl)piperazin-1-yl)ethyl)pyrrolidine-2,5-dione (22) - ${ }^{1} \mathrm{H}$ NMR

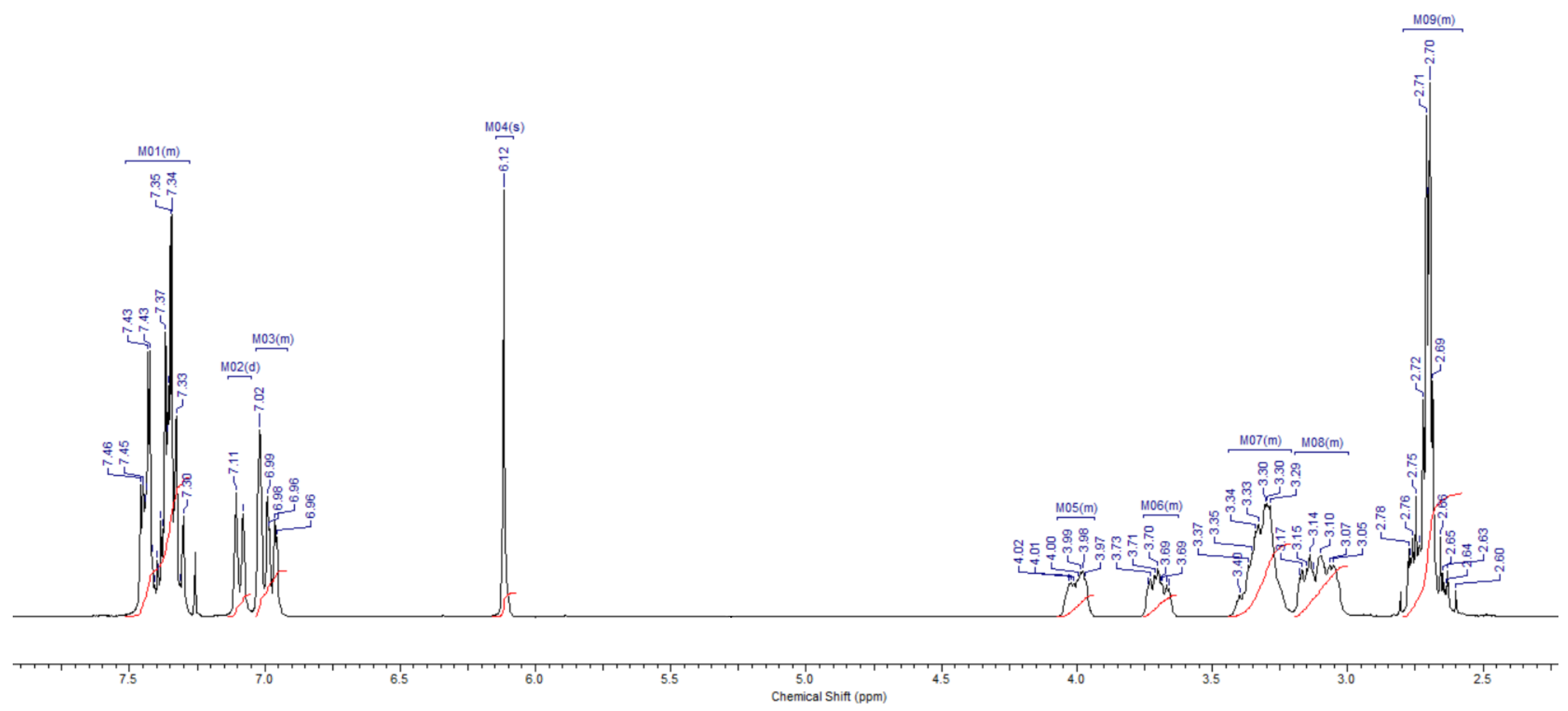


1-(2-Oxo-1-phenyl-2-(4-(3-(trifluoromethyl)phenyl)piperazin-1-yl)ethyl)pyrrolidine-2,5-dione (22) - ${ }^{13} \mathrm{C}$ NMR

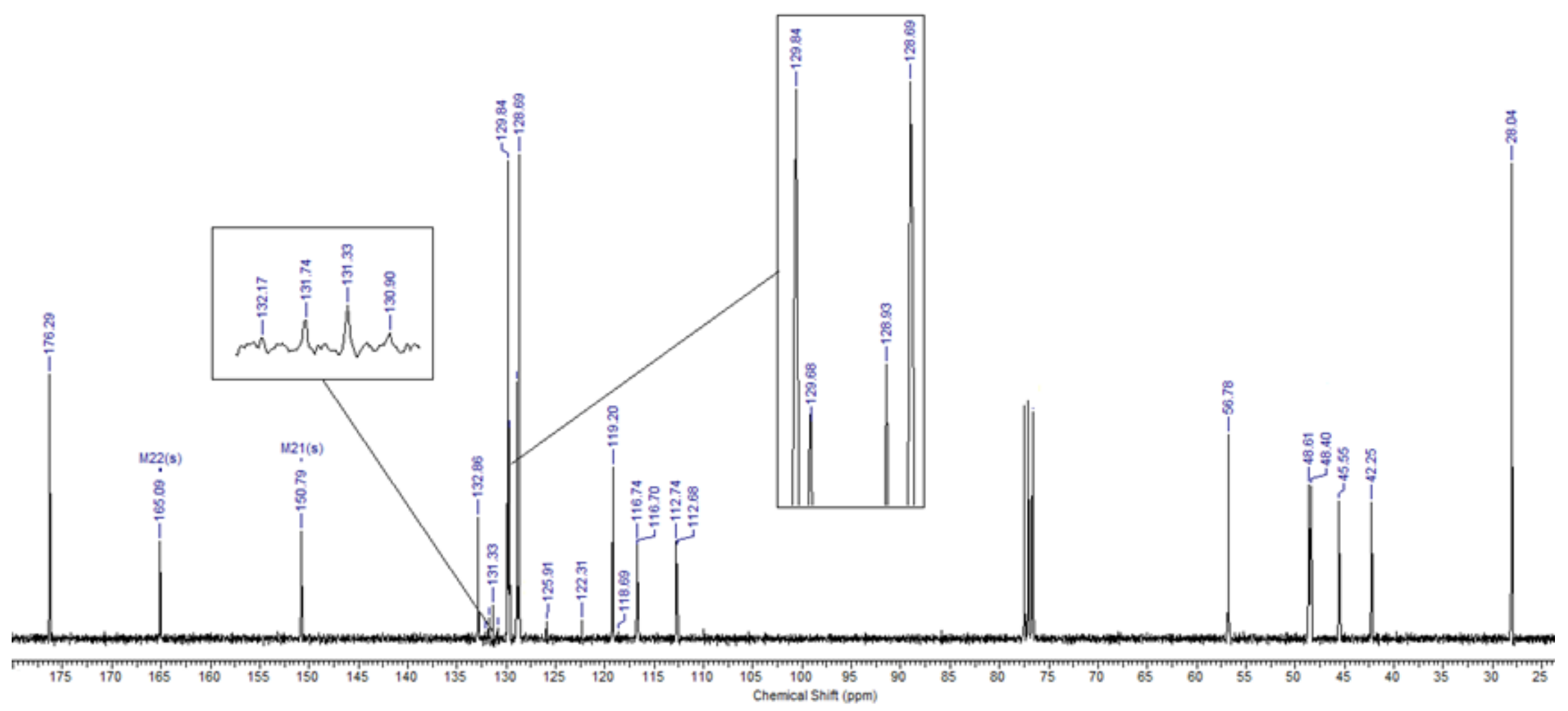


1-(2-Oxo-1-phenyl-2-(4-(3-(trifluoromethyl)phenyl)piperazin-1-yl)ethyl)pyrrolidine-2,5-dione (22) - ${ }^{19}$ F NMR

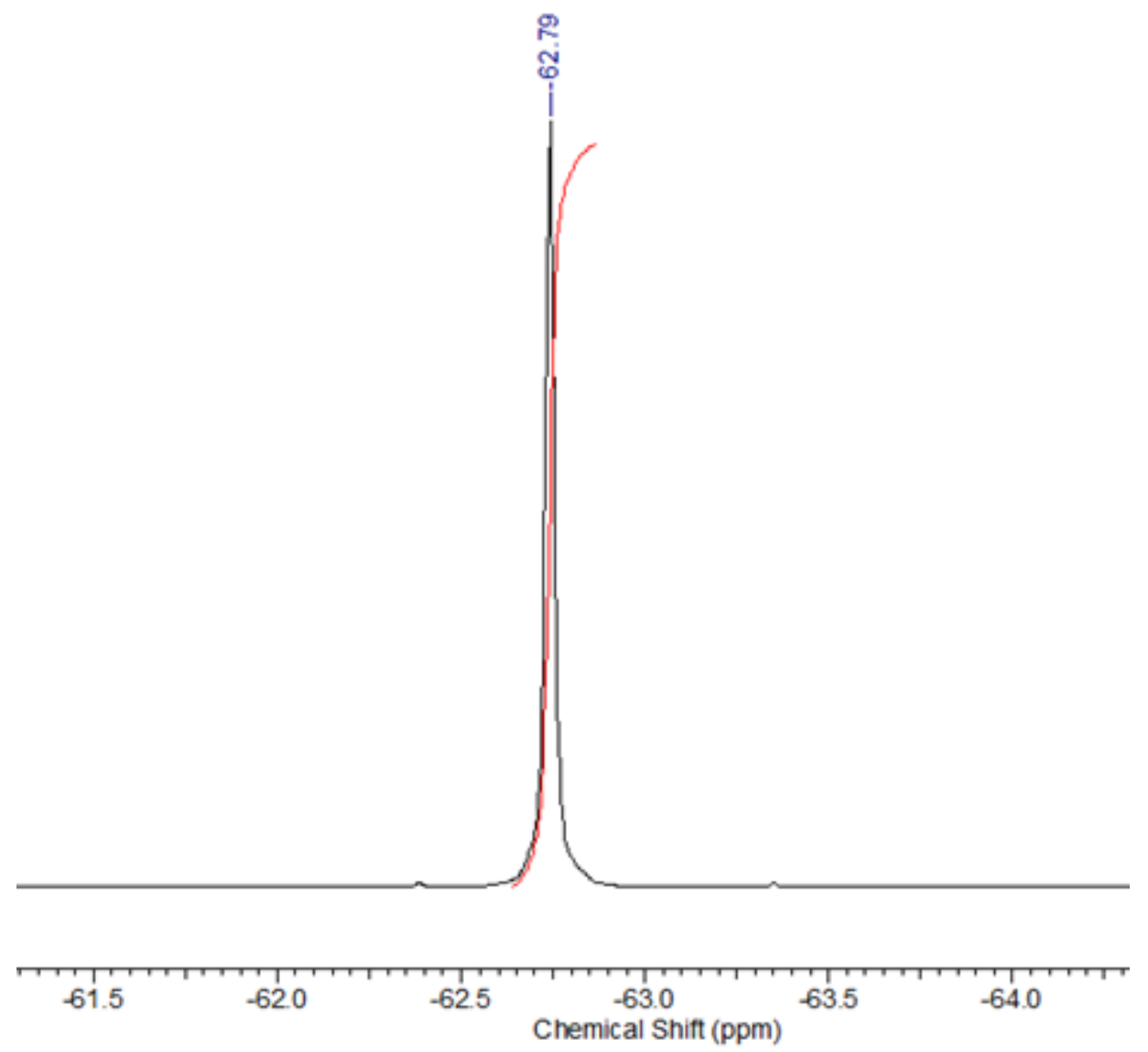


1-(2-Oxo-1-phenyl-2-(4-(4-(trifluoromethyl)phenyl)piperazin-1-yl)ethyl)pyrrolidine-2,5-dione (23) - ${ }^{1} \mathrm{H}$ NMR

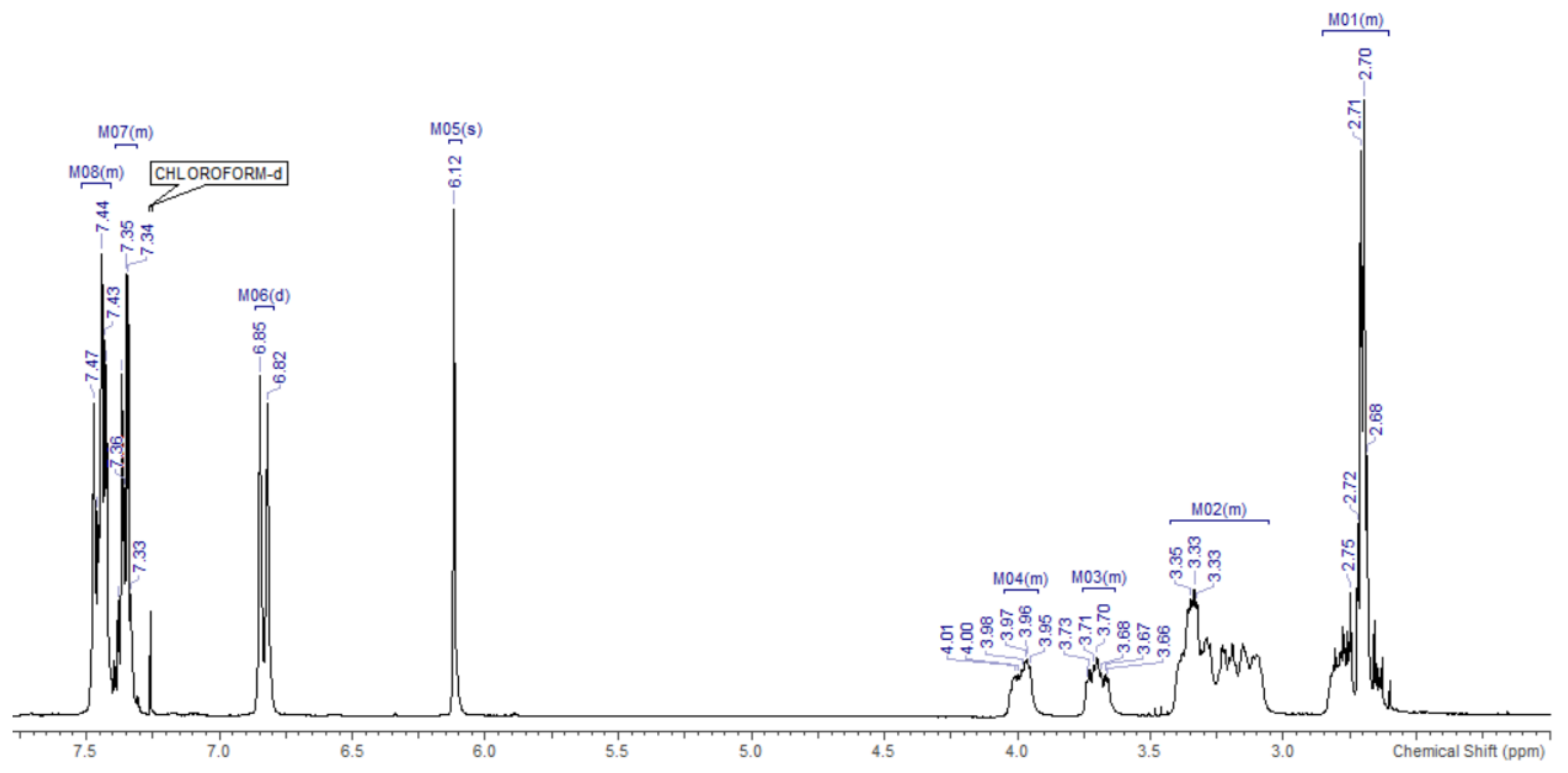


1-(2-Oxo-1-phenyl-2-(4-(4-(trifluoromethyl)phenyl)piperazin-1-yl)ethyl)pyrrolidine-2,5-dione (23) - ${ }^{13} \mathrm{C}$ NMR

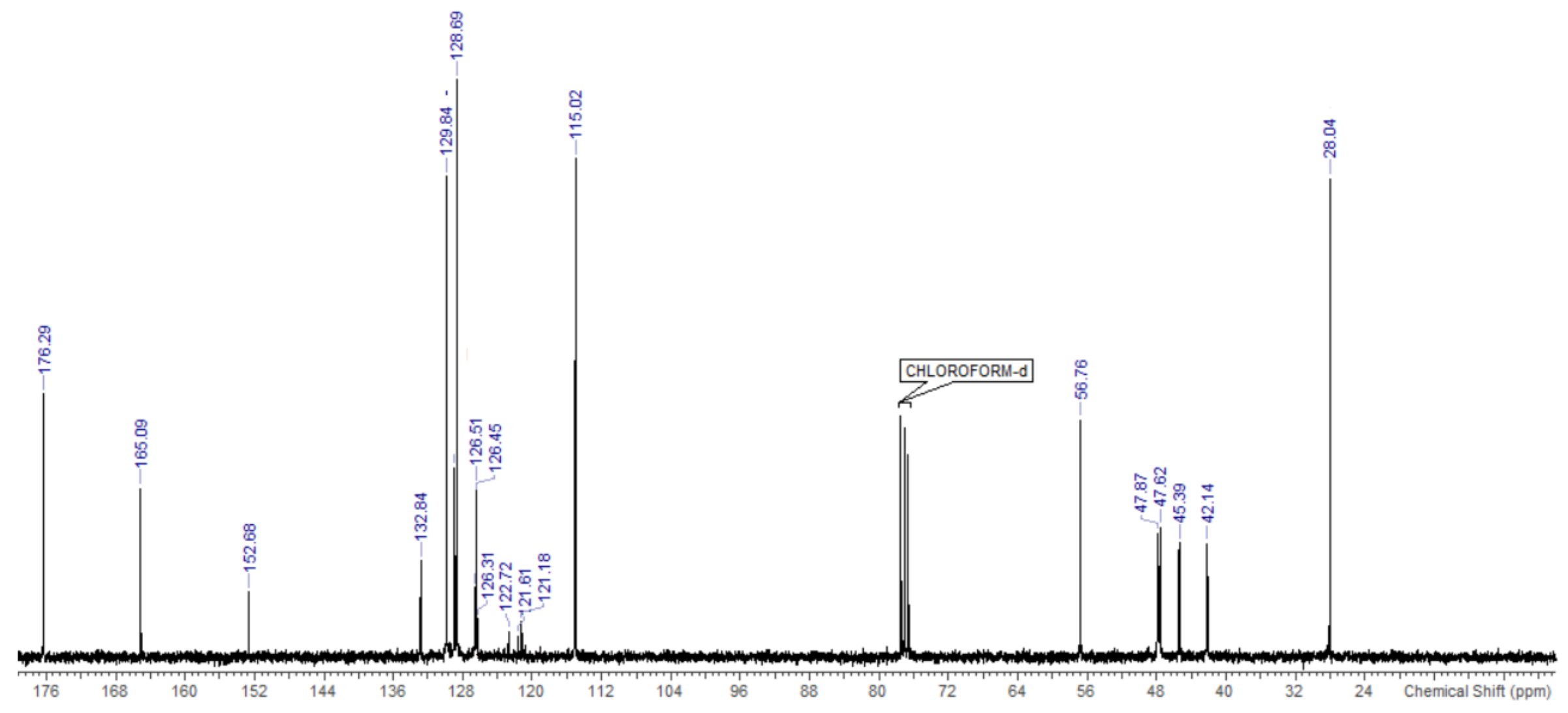


1-(2-(4-(3-Methoxyphenyl)piperazin-1-yl)-2-oxo-1-phenylethyl)pyrrolidine-2,5-dione (24) - ${ }^{1} \mathrm{H}$ NMR

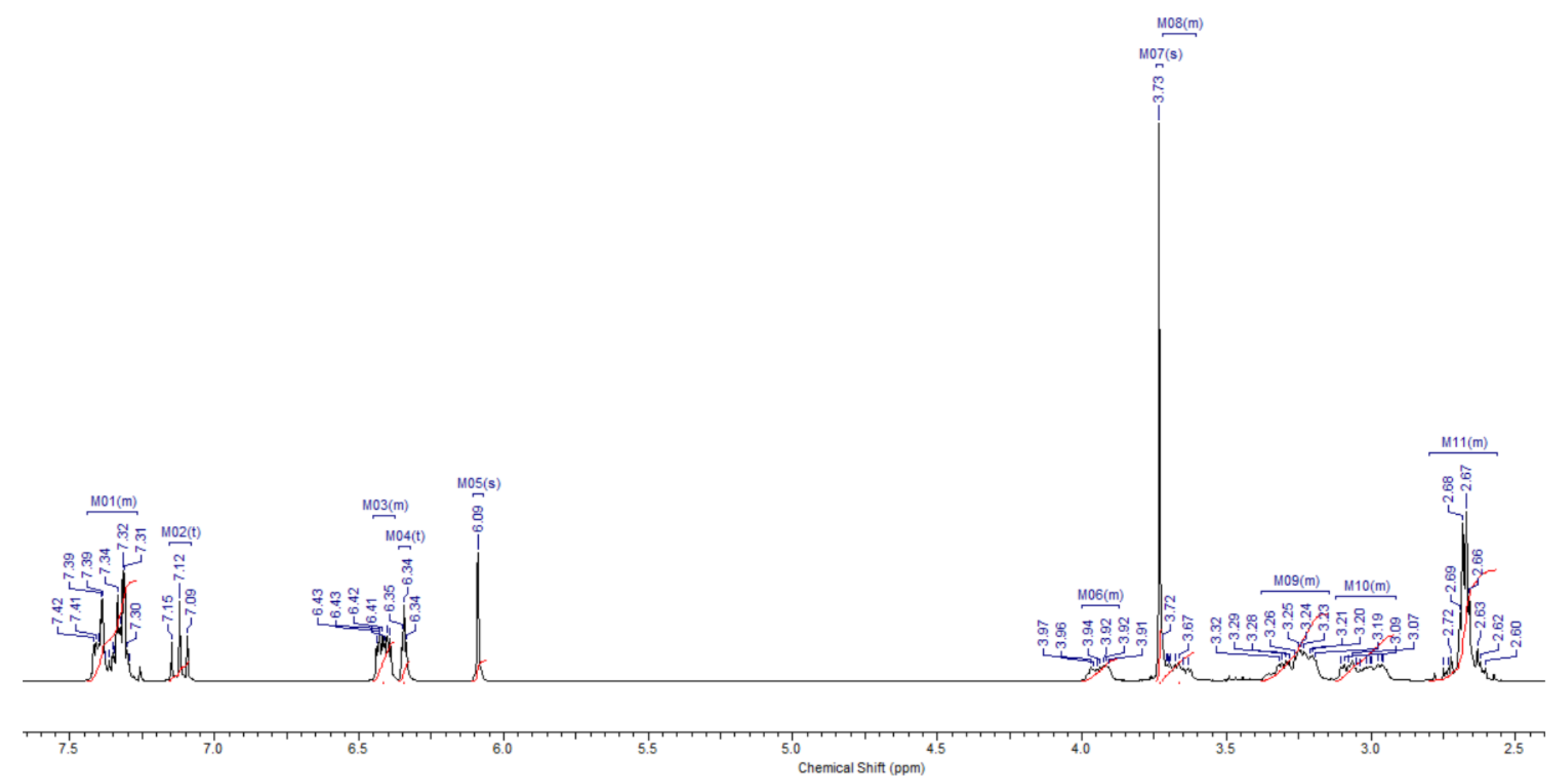


1-(2-(4-(3-Methoxyphenyl)piperazin-1-yl)-2-oxo-1-phenylethyl)pyrrolidine-2,5-dione (24) - ${ }^{13} \mathrm{C}$ NMR

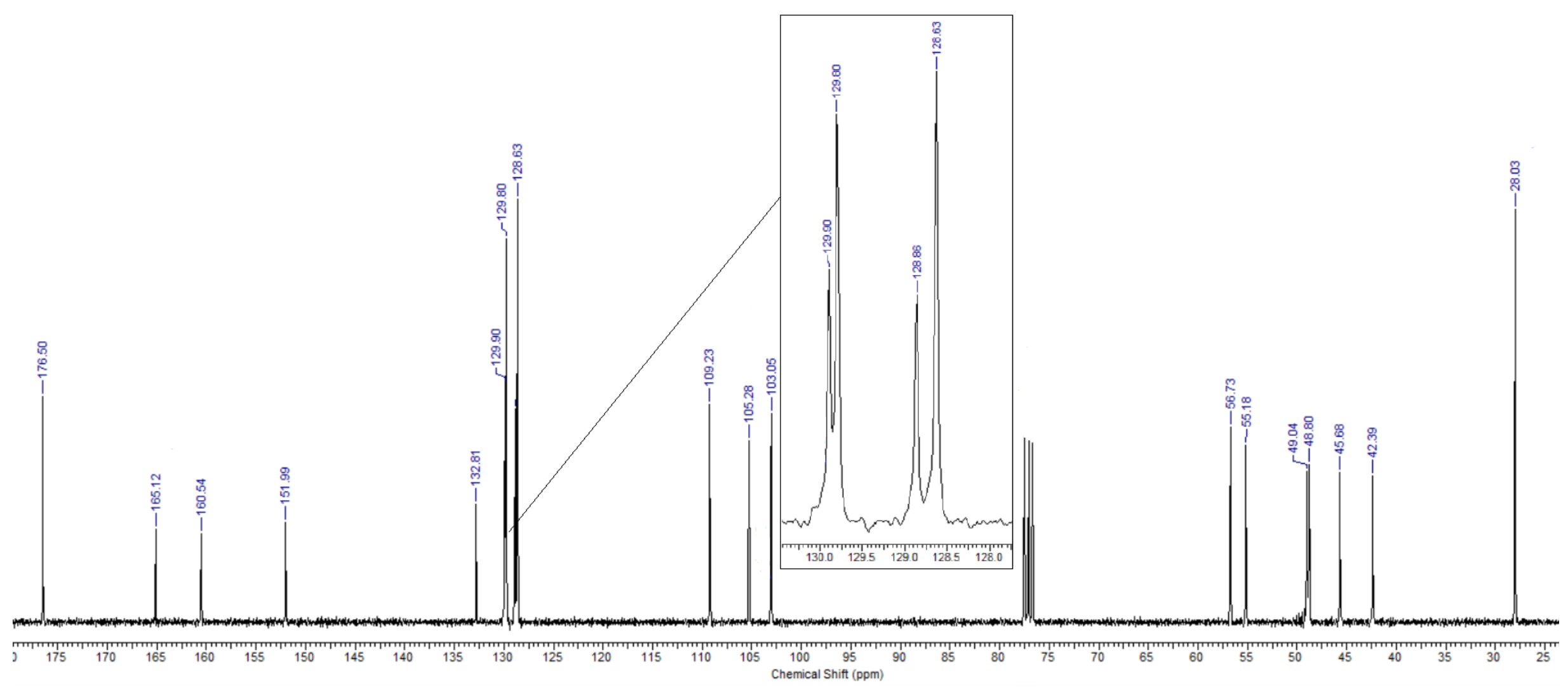


1-(2-Oxo-1-phenyl-2-(4-(3-(trifluoromethoxy)phenyl)piperazin-1-yl)ethyl)pyrrolidine-2,5-dione (25) - ${ }^{1} \mathrm{H}$ NMR

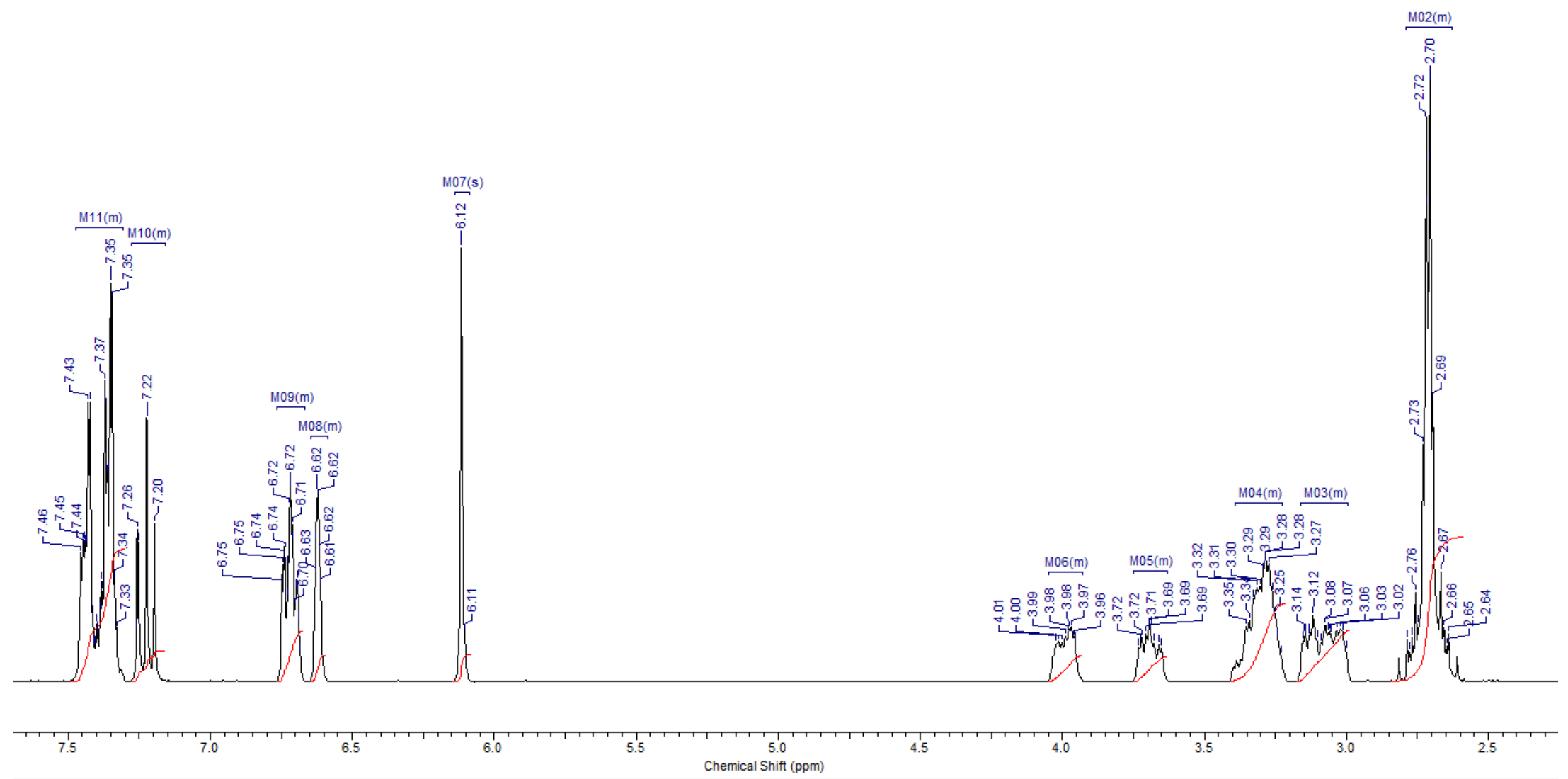


1-(2-Oxo-1-phenyl-2-(4-(3-(trifluoromethoxy)phenyl)piperazin-1-yl)ethyl)pyrrolidine-2,5-dione (25) - ${ }^{13} \mathrm{C}$ NMR
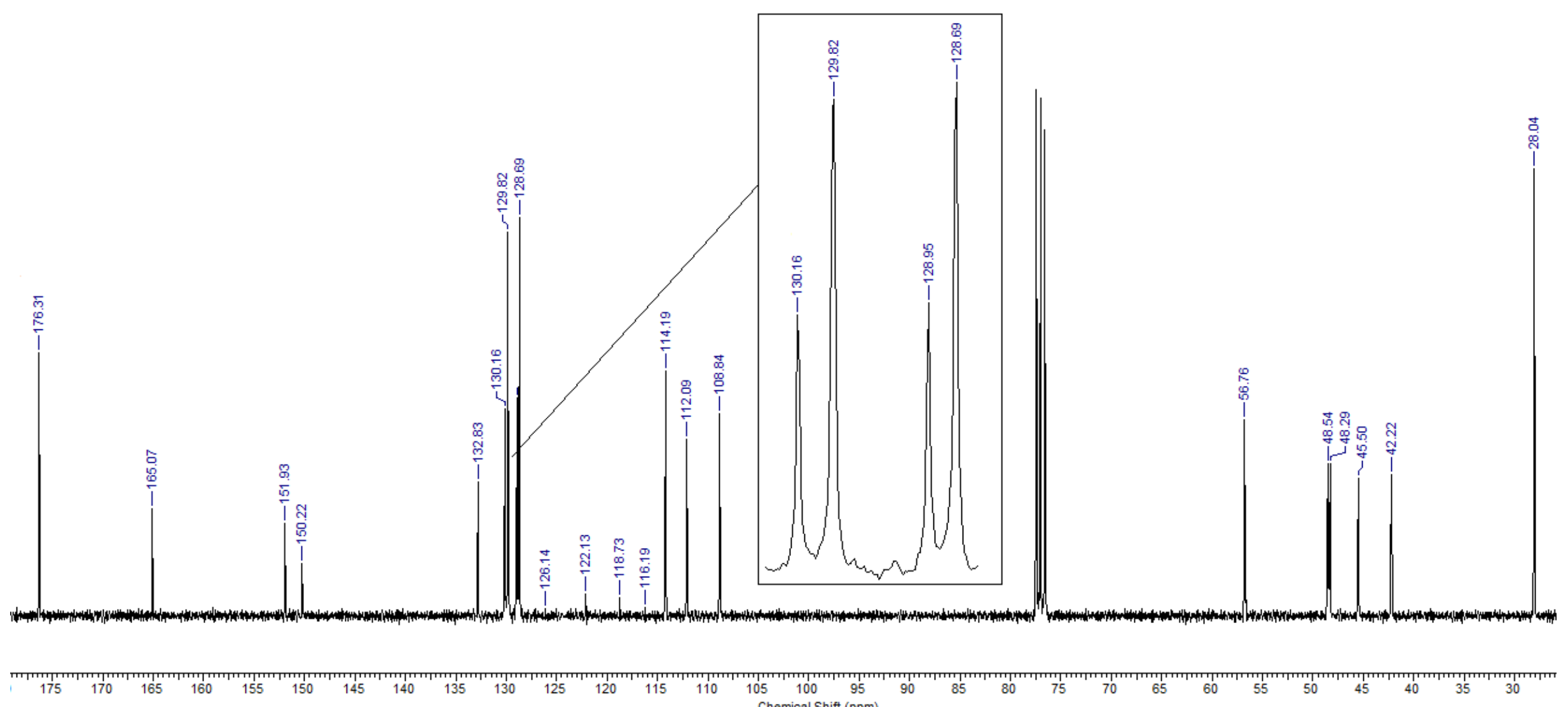
1-(2-(4-(3-(Methylthio)phenyl)piperazin-1-yl)-2-oxo-1-phenylethyl)pyrrolidine-2,5-dione (26) - ${ }^{1} \mathrm{H}$ NMR

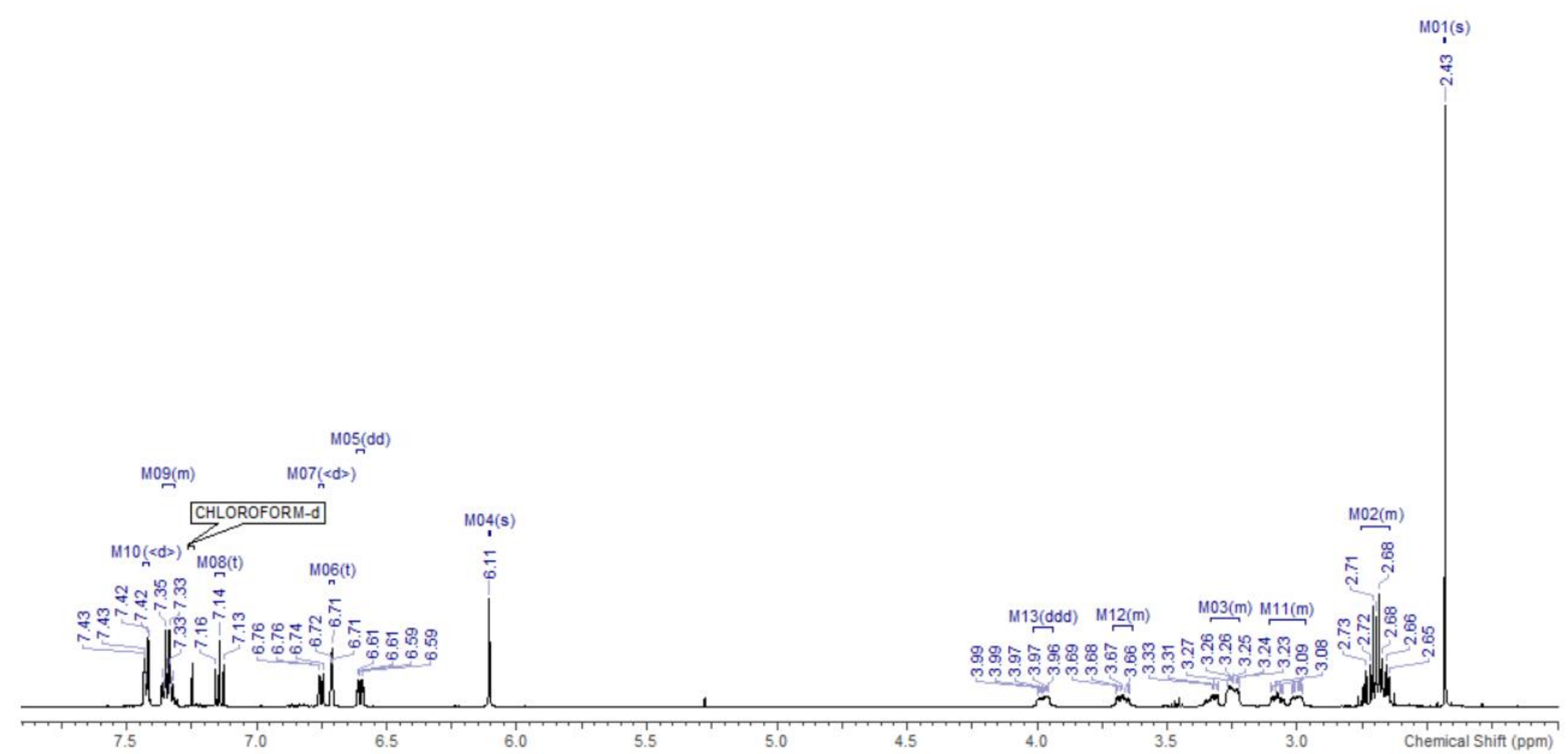


1-(2-(4-(3-(Methylthio)phenyl)piperazin-1-yl)-2-oxo-1-phenylethyl)pyrrolidine-2,5-dione (26) - ${ }^{13} \mathrm{C}$ NMR

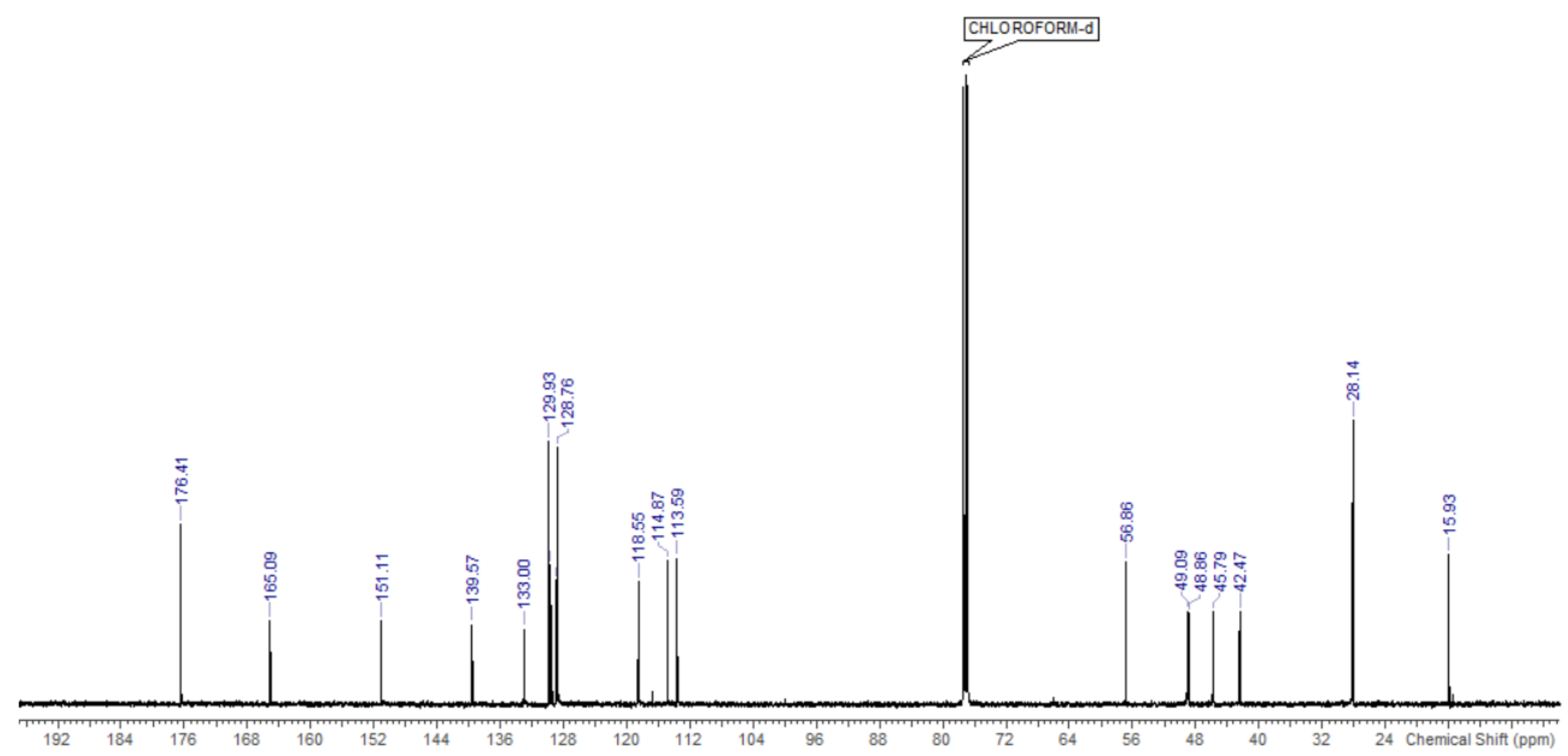


1-(2-Oxo-1-phenyl-2-(4-(3-((trifluoromethyl)thio)phenyl)piperazin-1-yl)ethyl)pyrrolidine-2,5-dione (27) - ${ }^{1} \mathrm{H}$ NMR

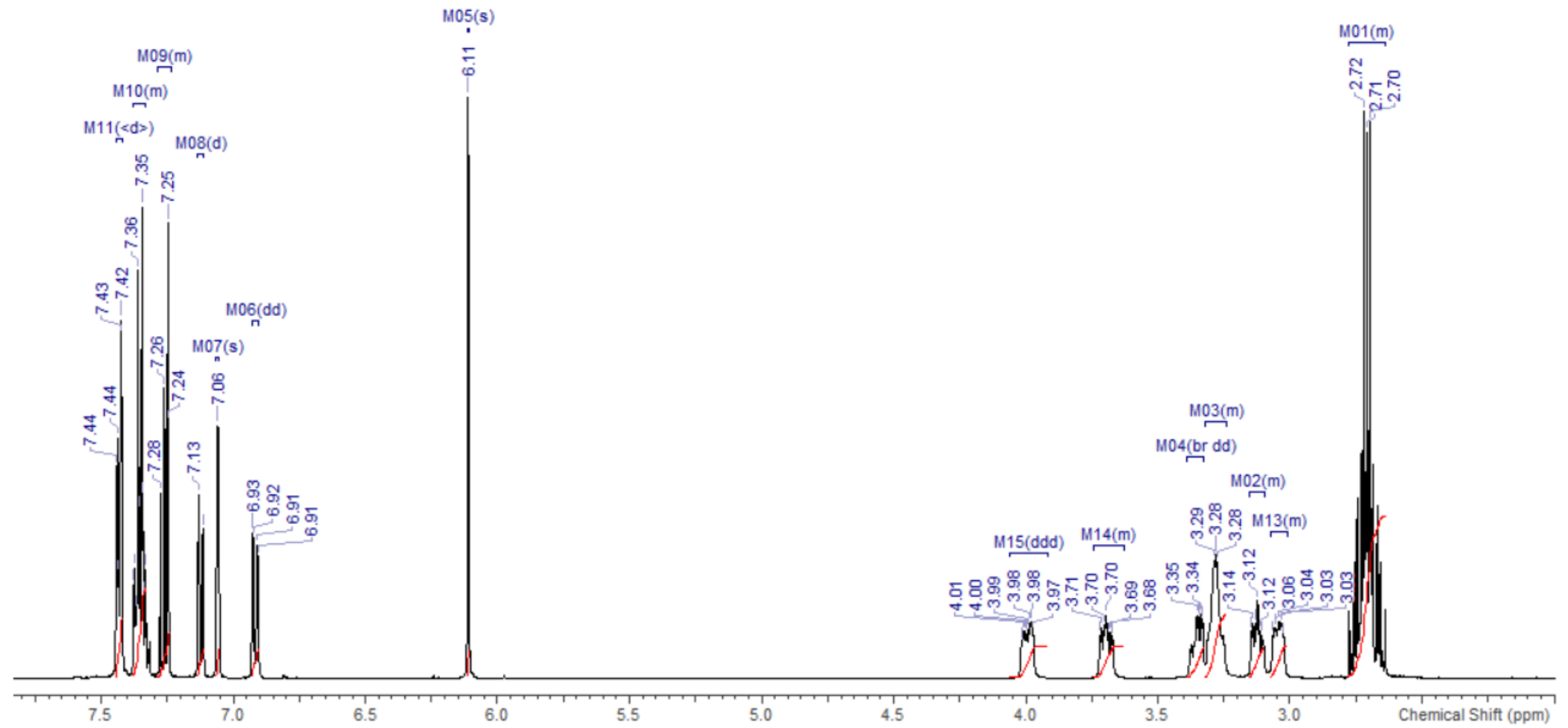


1-(2-Oxo-1-phenyl-2-(4-(3-((trifluoromethyl)thio)phenyl)piperazin-1-yl)ethyl)pyrrolidine-2,5-dione (27) - ${ }^{13} \mathrm{C}$ NMR

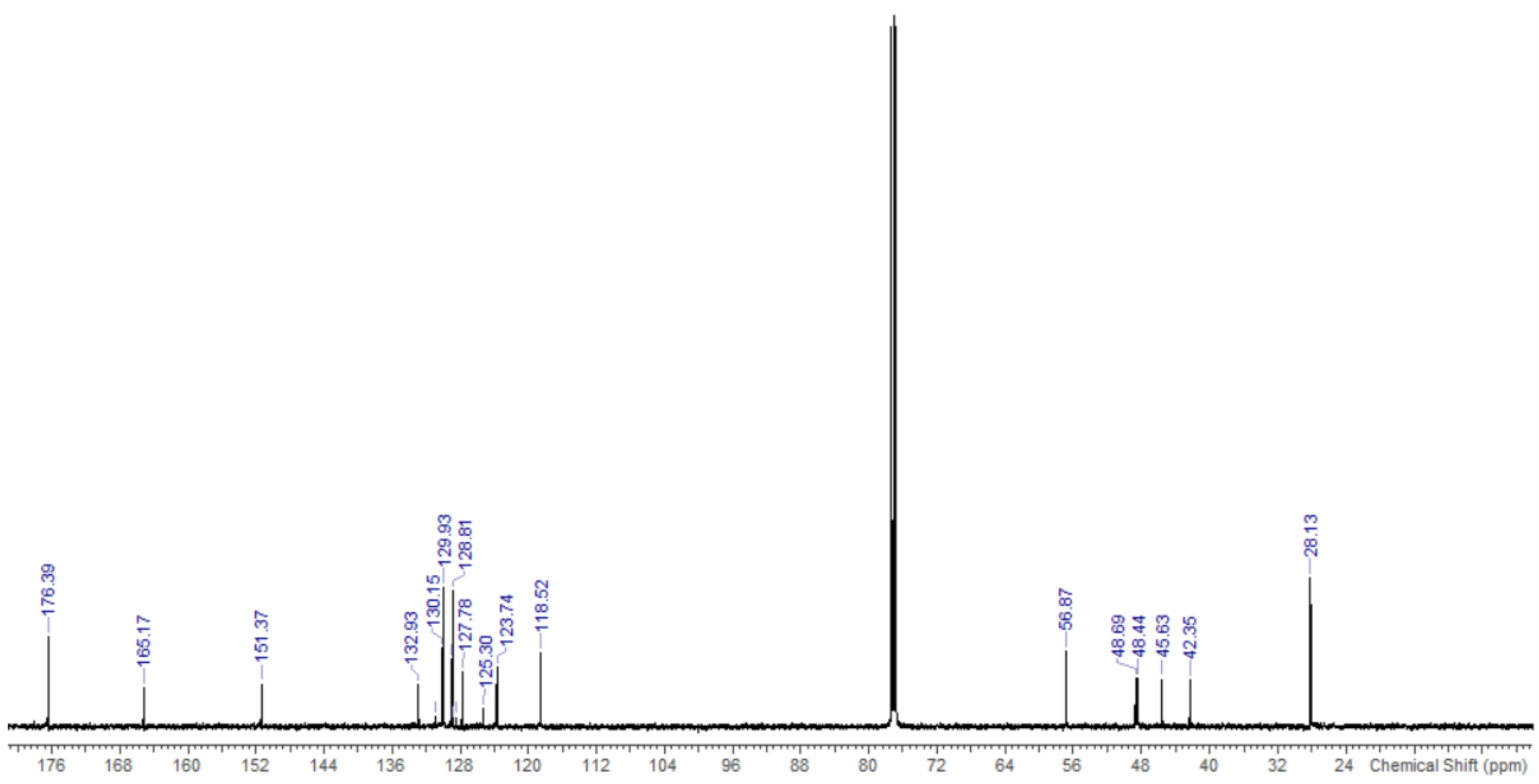


1-(2-(4-(3-(Difluoromethyl)phenyl)piperazin-1-yl)-2-oxo-1-phenylethyl)pyrrolidine-2,5-dione (28) - ${ }^{1} \mathrm{H}$ NMR

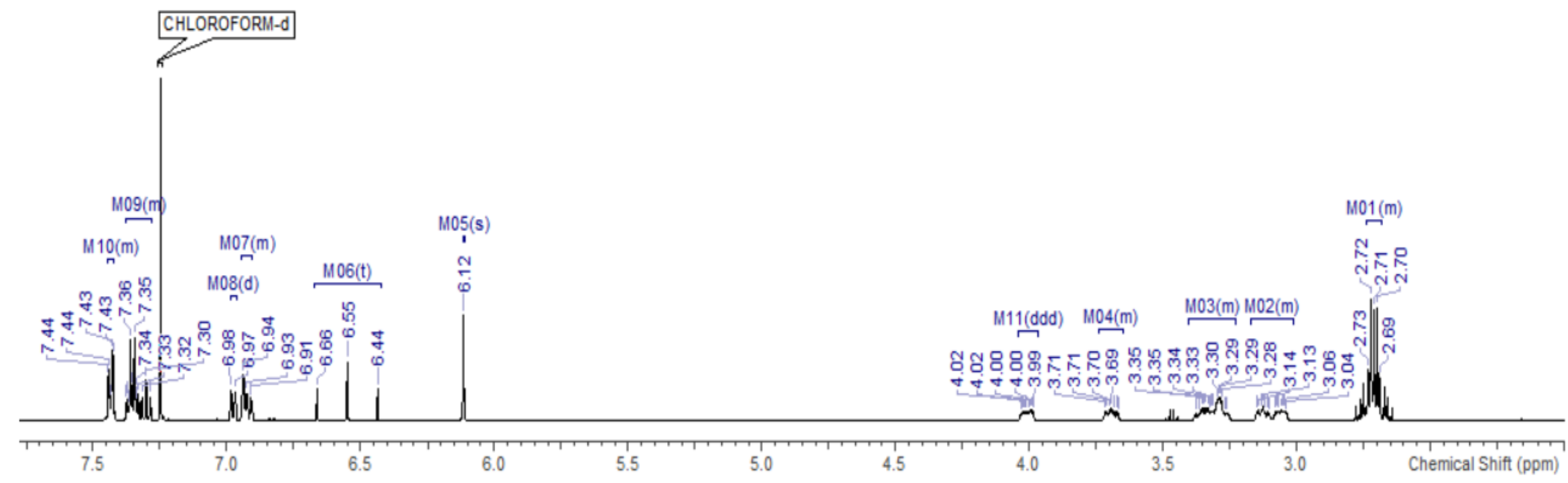


1-(2-(4-(3-(Difluoromethyl)phenyl)piperazin-1-yl)-2-oxo-1-phenylethyl)pyrrolidine-2,5-dione (28) - ${ }^{13} \mathrm{C}$ NMR

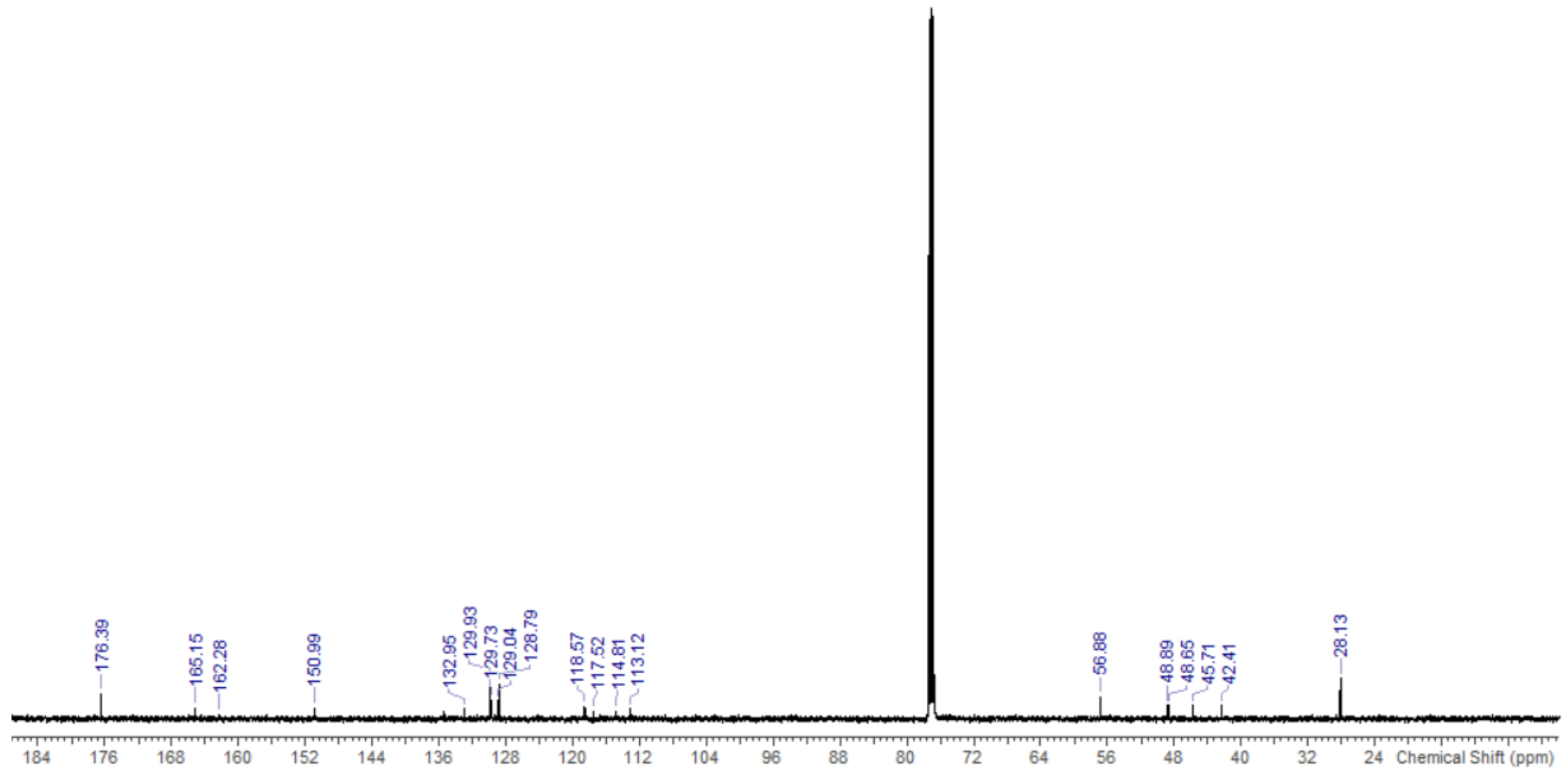


1-(2-(4-(3-Aminophenyl)piperazin-1-yl)-2-oxo-1-phenylethyl)pyrrolidine-2,5-dione (29) - ${ }^{1} \mathrm{H}$ NMR

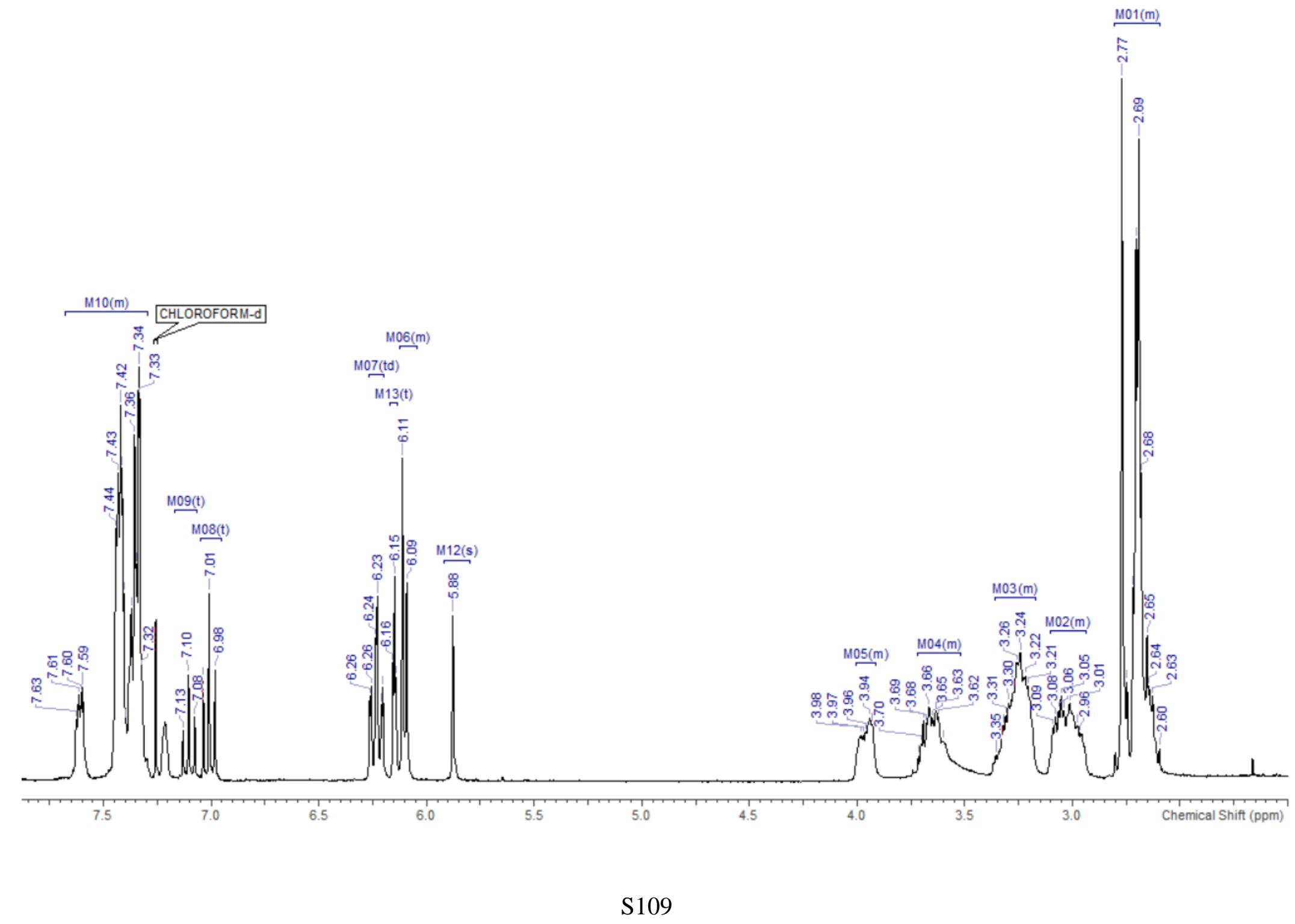


1-(2-(4-(3-Aminophenyl)piperazin-1-yl)-2-oxo-1-phenylethyl)pyrrolidine-2,5-dione (29) - ${ }^{13} \mathrm{C}$ NMR

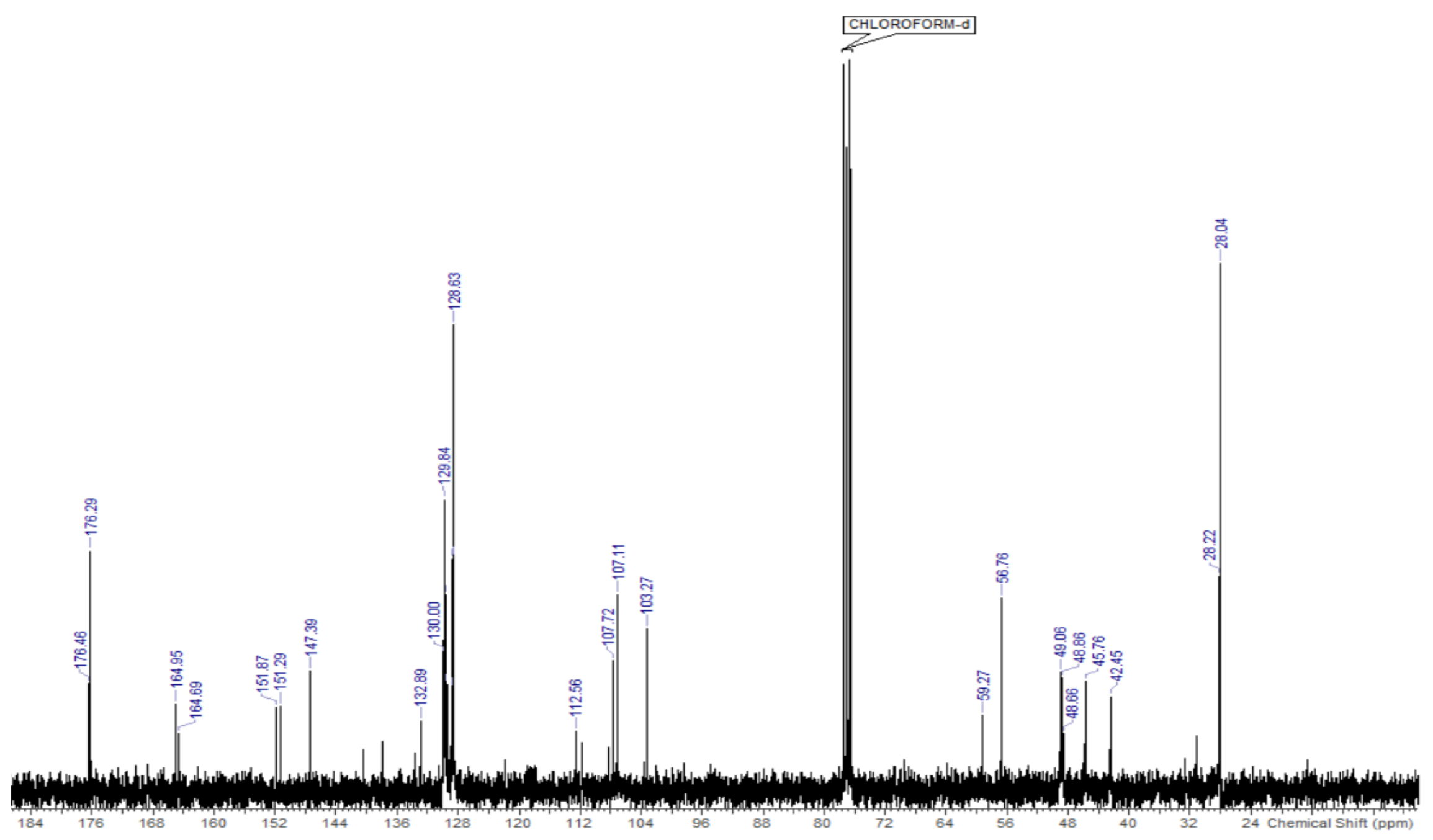


1-(2-(4-(3-Isopropylphenyl)piperazin-1-yl)-2-oxo-1-phenylethyl)pyrrolidine-2,5-dione (30) - ${ }^{1} \mathrm{H}$ NMR

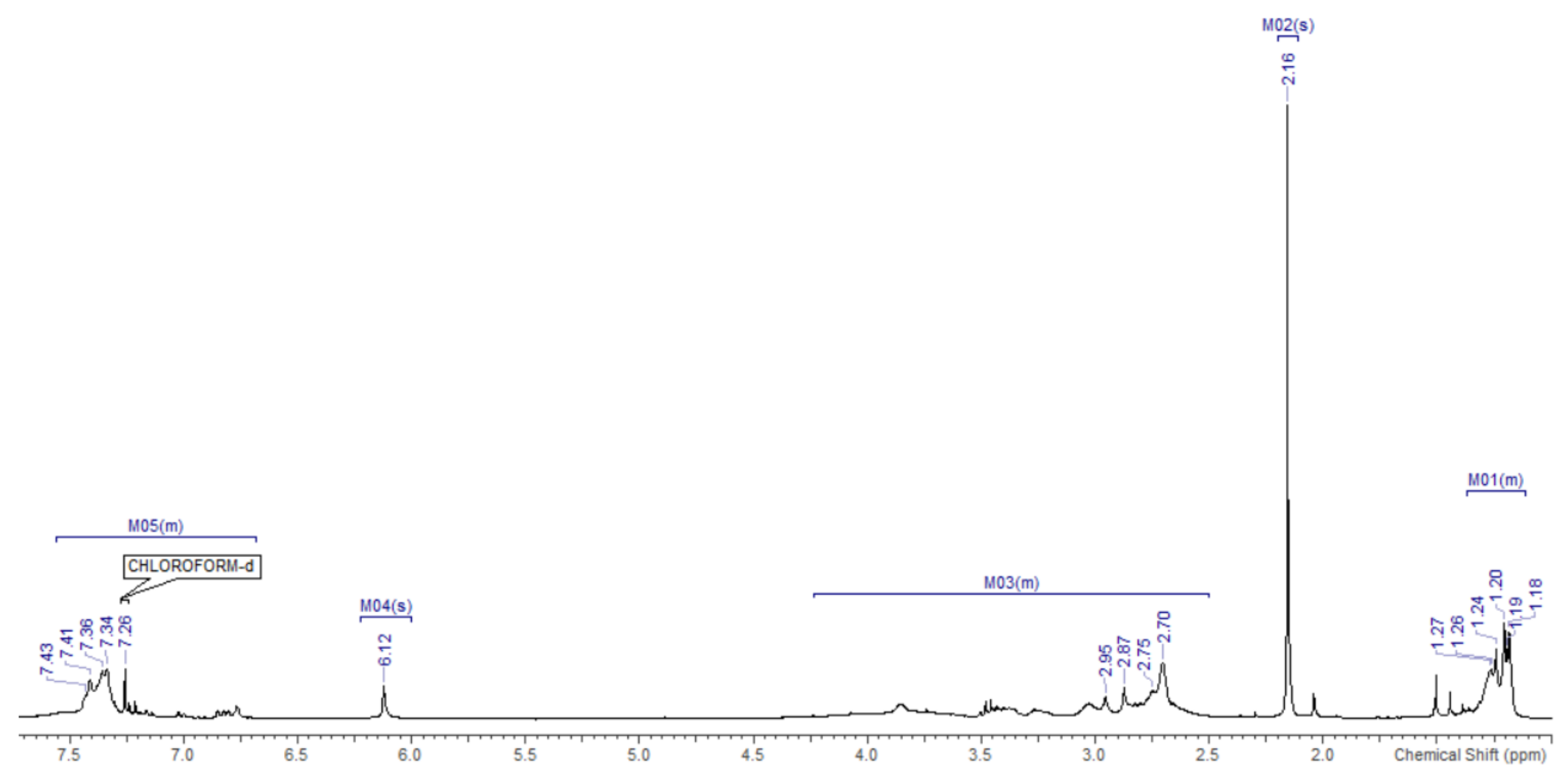


1-(2-(4-(3-Isopropylphenyl)piperazin-1-yl)-2-oxo-1-phenylethyl)pyrrolidine-2,5-dione (30) - ${ }^{13} \mathrm{C}$ NMR

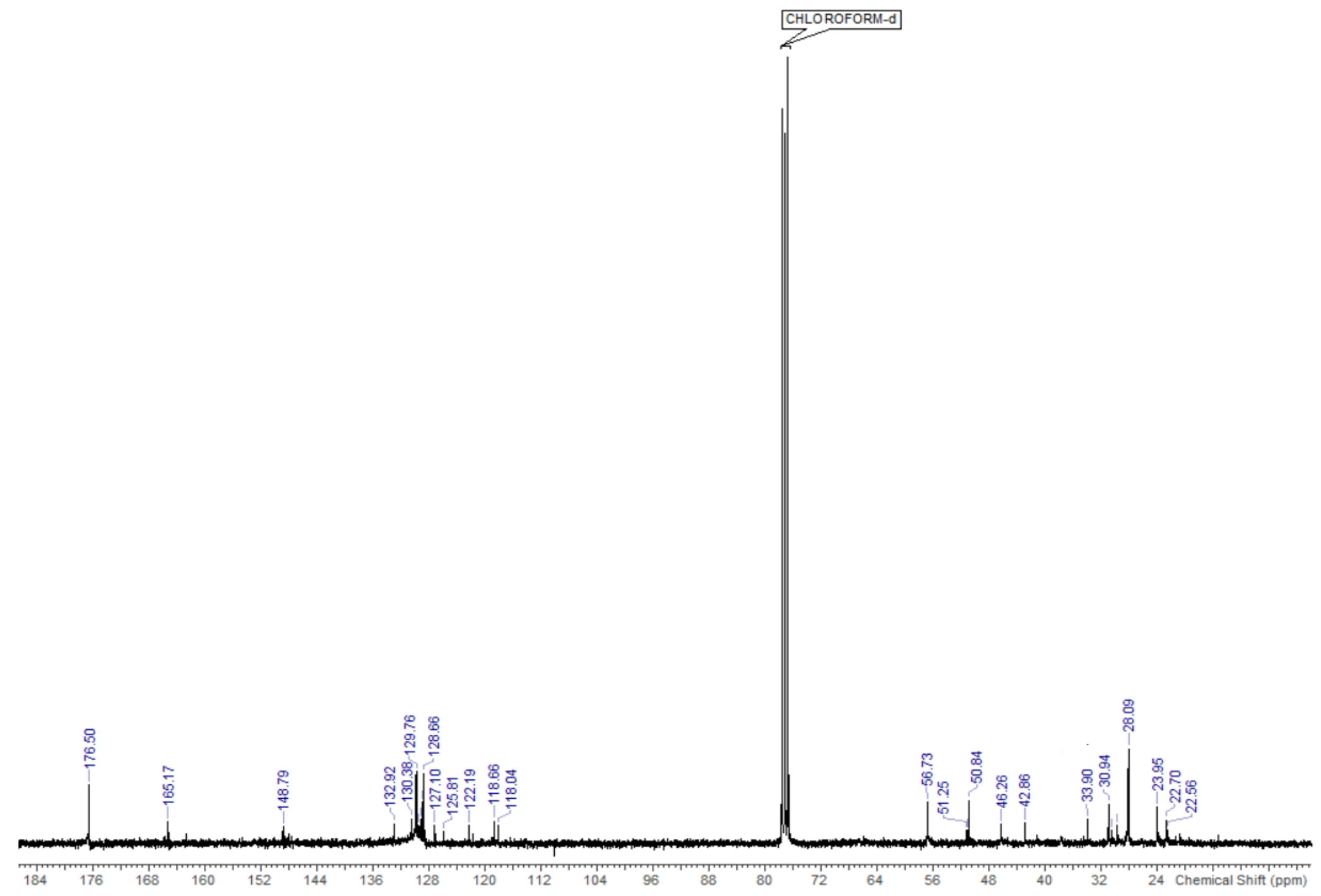


1-(2-(4-(3-(tert-Butyl)phenyl)piperazin-1-yl)-2-oxo-1-phenylethyl)pyrrolidine-2,5-dione (31) - ${ }^{1} \mathrm{H}$ NMR

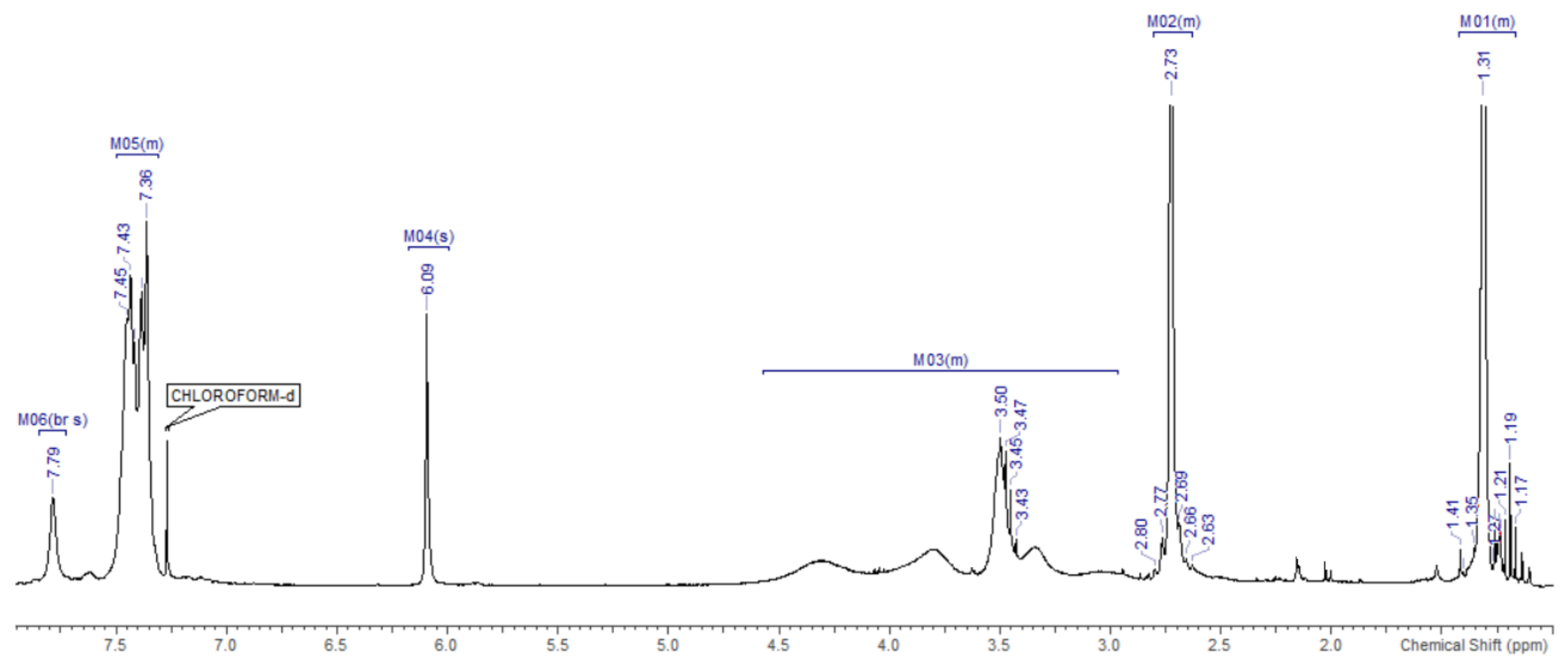


1-(2-(4-(3-(tert-Butyl)phenyl)piperazin-1-yl)-2-oxo-1-phenylethyl)pyrrolidine-2,5-dione (31) - ${ }^{13} \mathrm{C}$ NMR

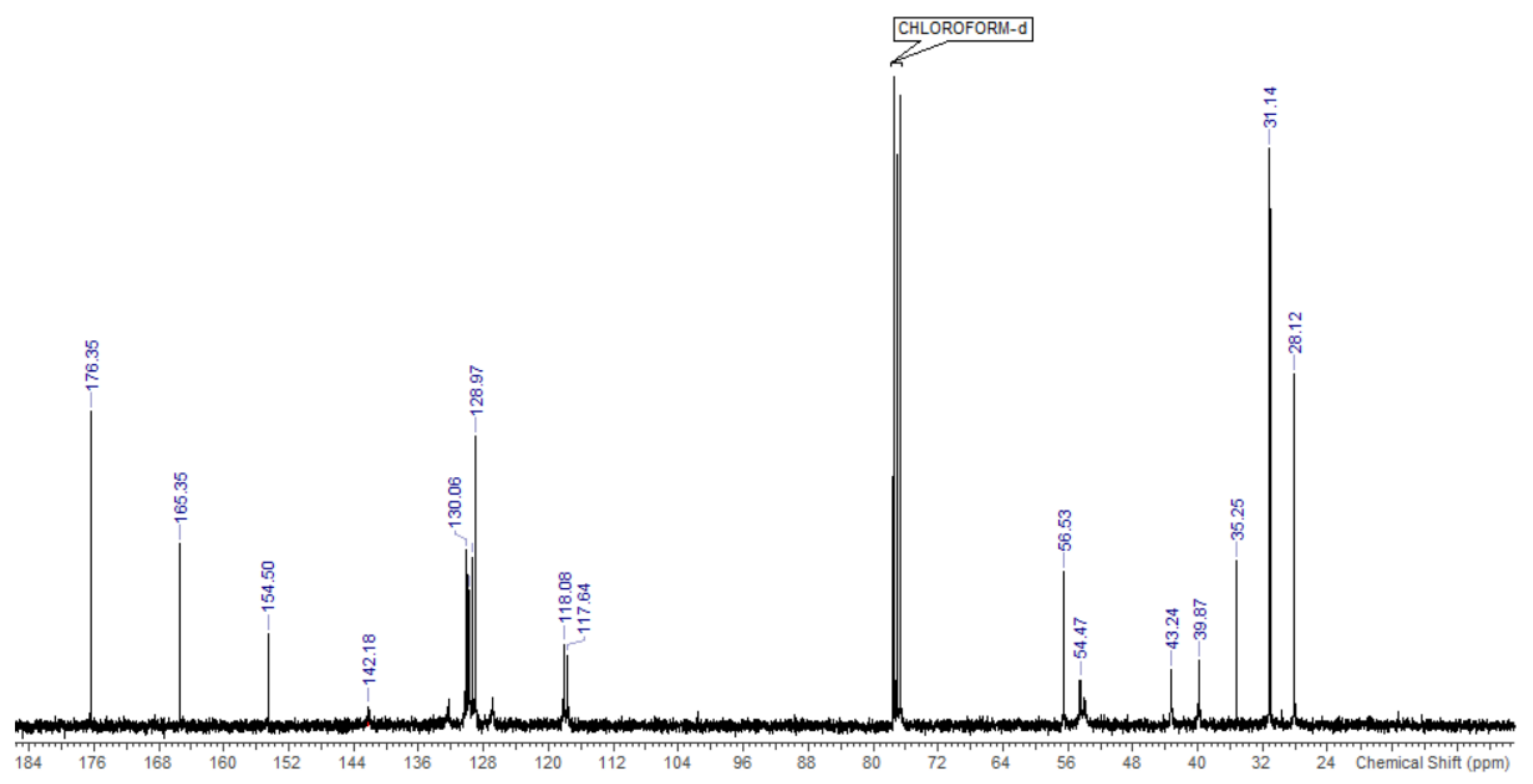


1-(2-(4-([1,1'-Biphenyl]-3-yl)piperazin-1-yl)-2-oxo-1-phenylethyl)pyrrolidine-2,5-dione (32) - ${ }^{1} \mathrm{H}$ NMR

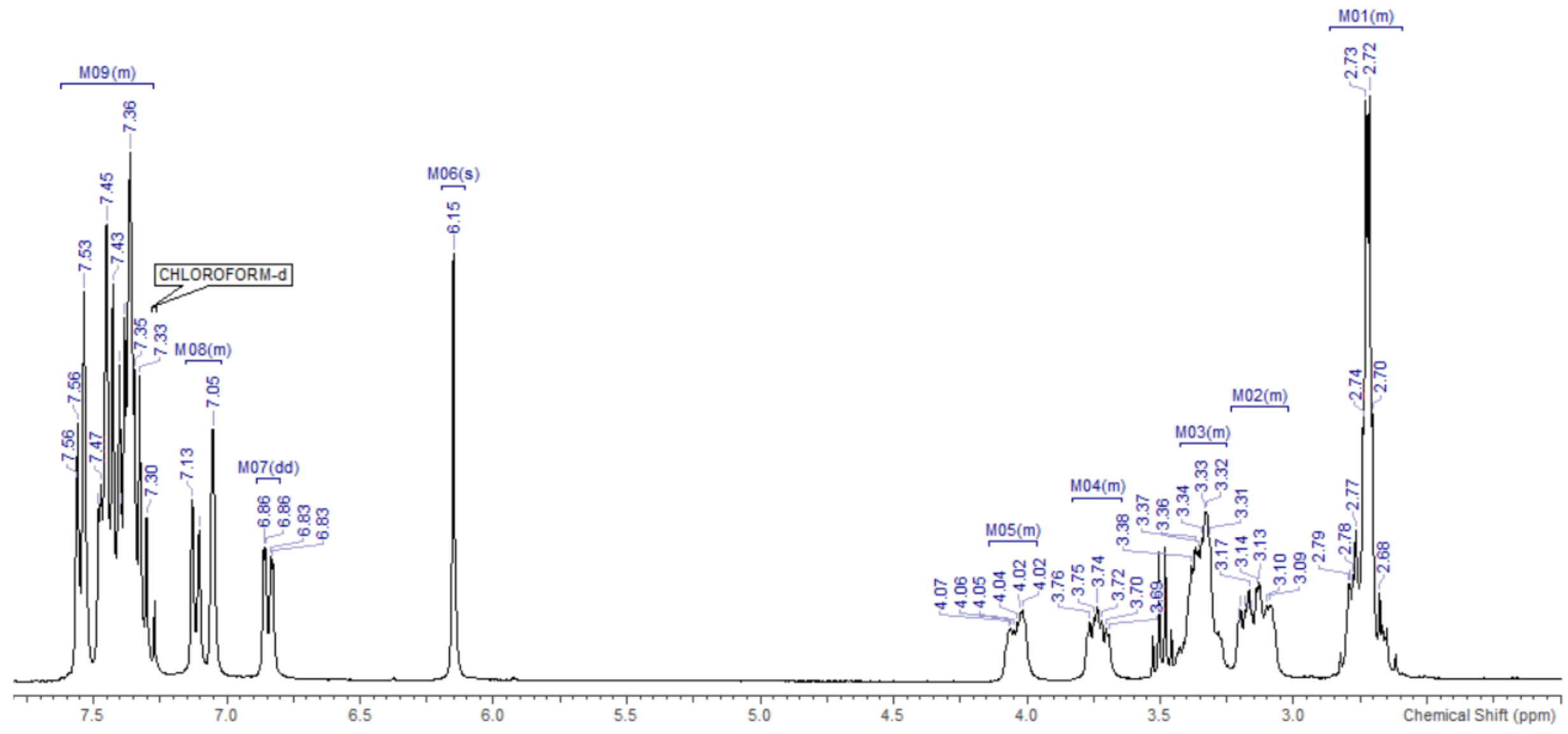


1-(2-(4-([1,1'-Biphenyl]-3-yl)piperazin-1-yl)-2-oxo-1-phenylethyl)pyrrolidine-2,5-dione $(32)-{ }^{13} \mathrm{C}$ NMR

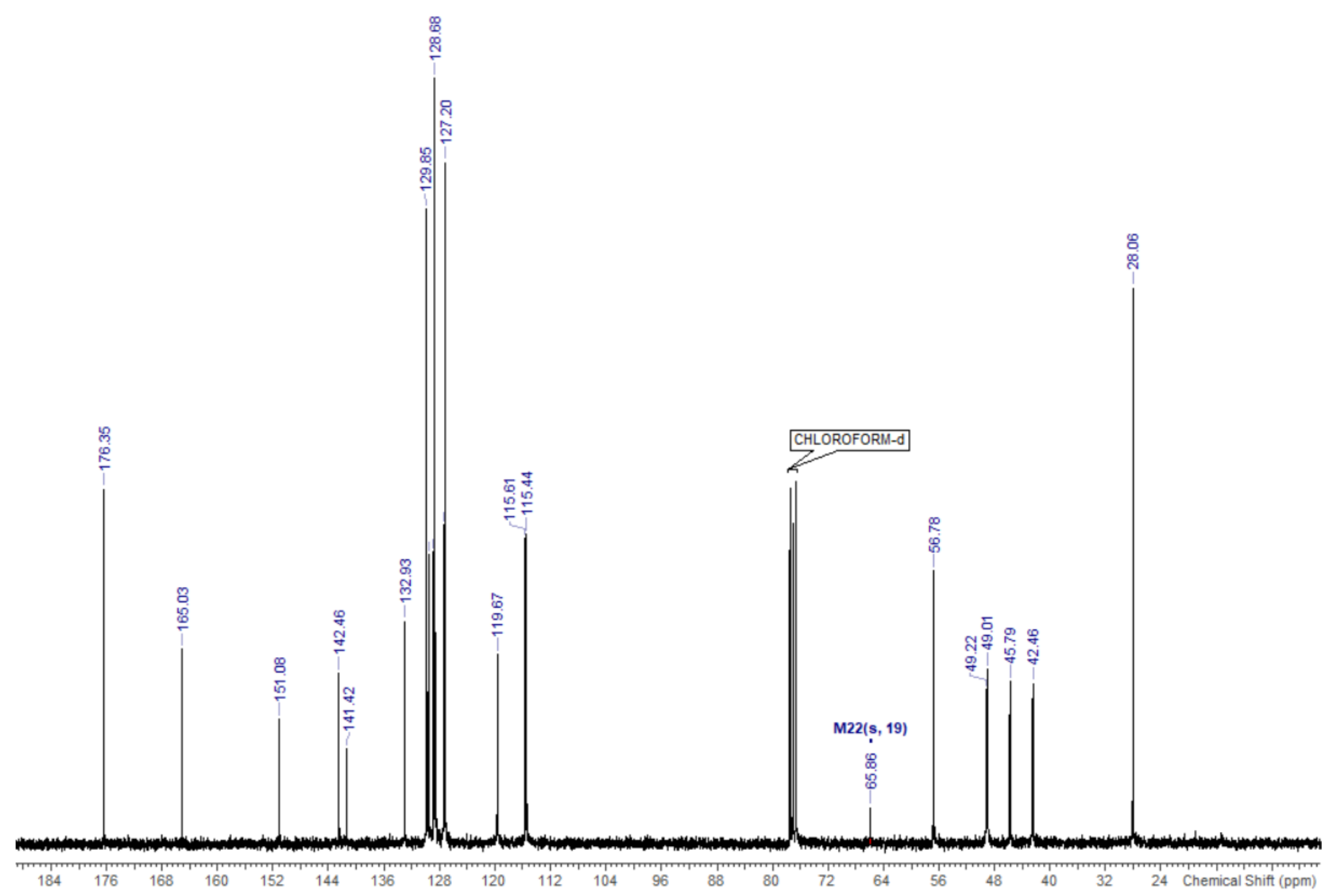


1-(2-(4-(3-(Benzyloxy)phenyl)piperazin-1-yl)-2-oxo-1-phenylethyl)pyrrolidine-2,5-dione (33) - ${ }^{1} \mathrm{H}$ NMR

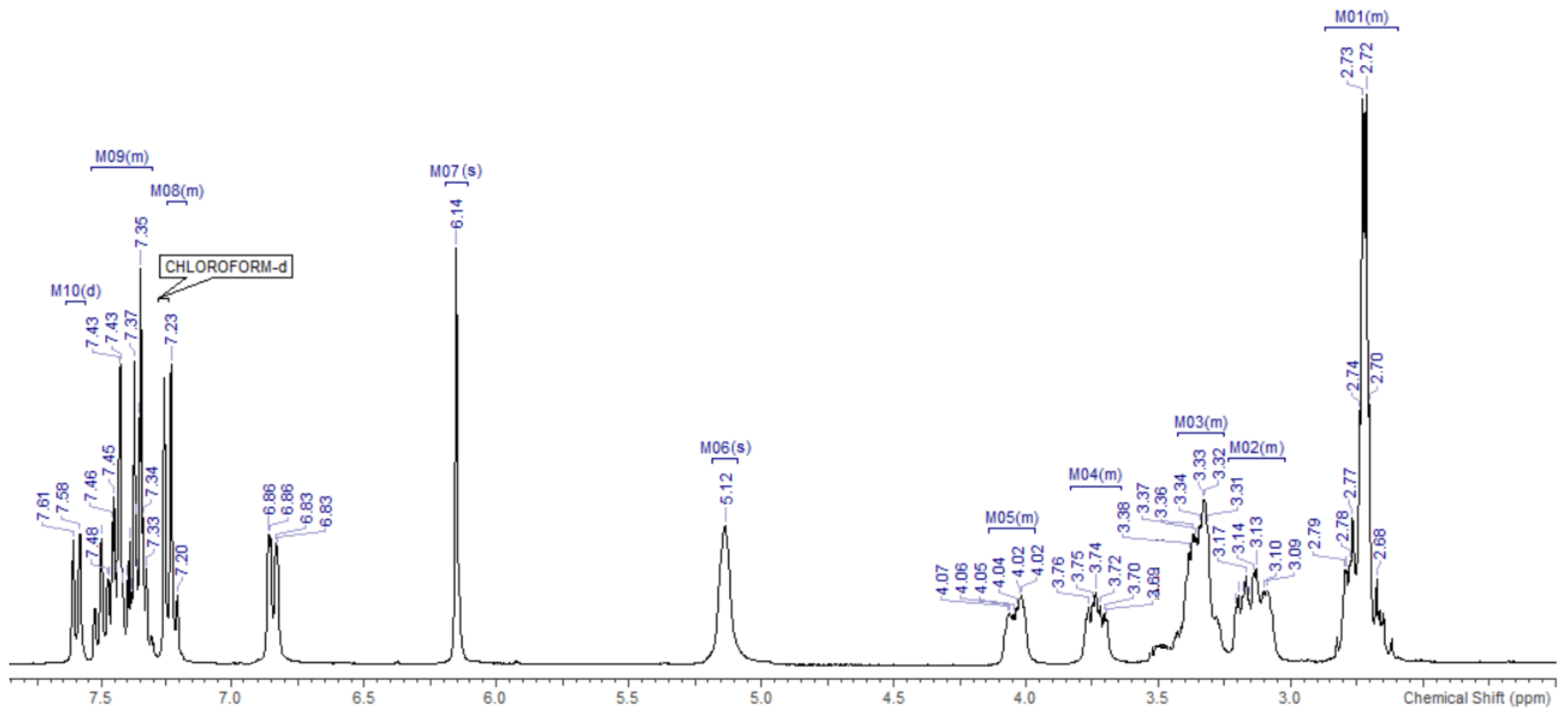


1-(1-(3-Fluorophenyl)-2-oxo-2-(4-(3-(trifluoromethyl)phenyl)piperazin-1-yl)ethyl)pyrrolidine-2,5-dione (34) - ${ }^{1} \mathrm{H}$ NMR

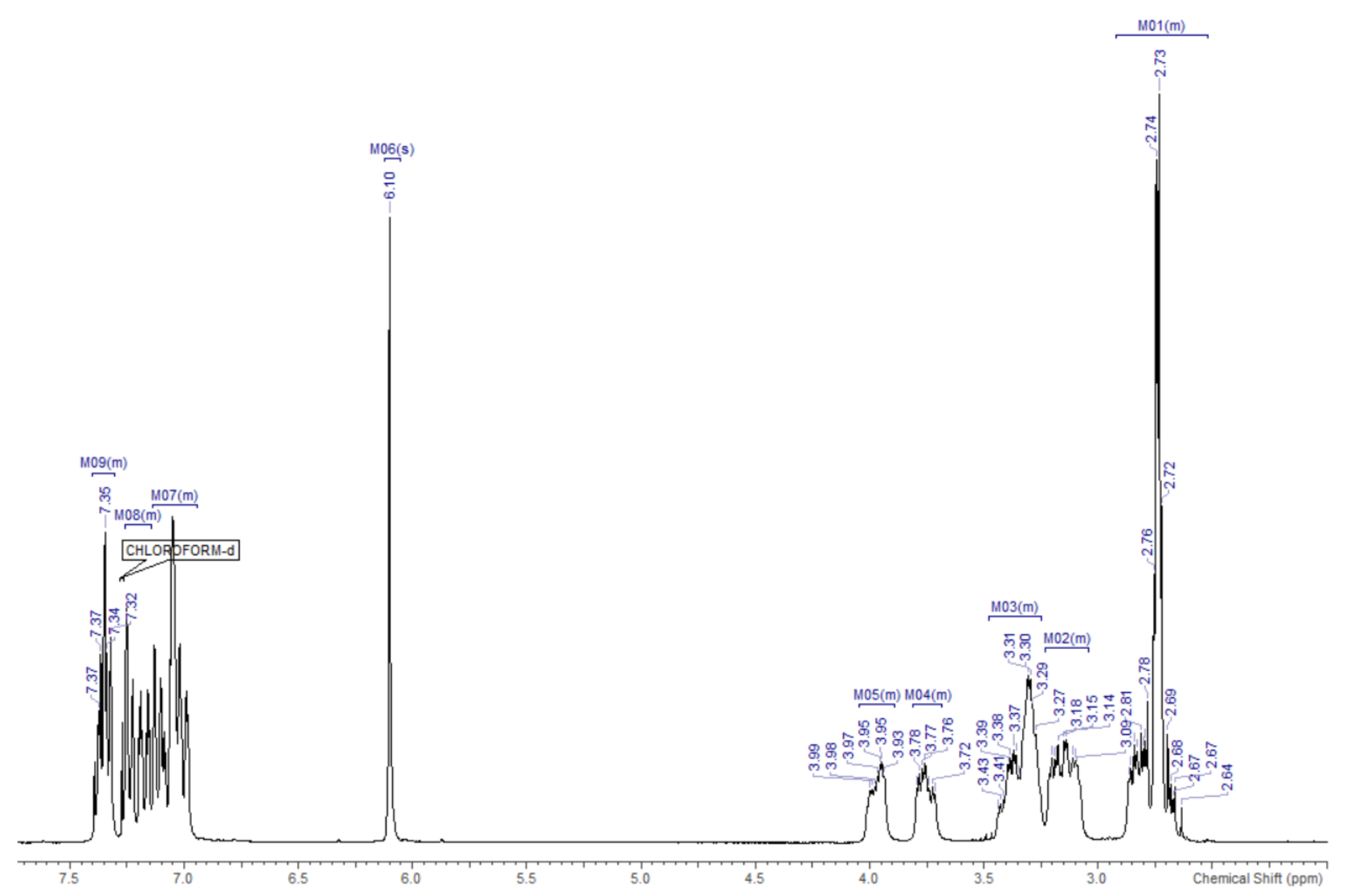


1-(1-(3-Fluorophenyl)-2-oxo-2-(4-(3-(trifluoromethyl)phenyl)piperazin-1-yl)ethyl)pyrrolidine-2,5-dione (34) - ${ }^{13} \mathrm{C} N \mathrm{NR}$

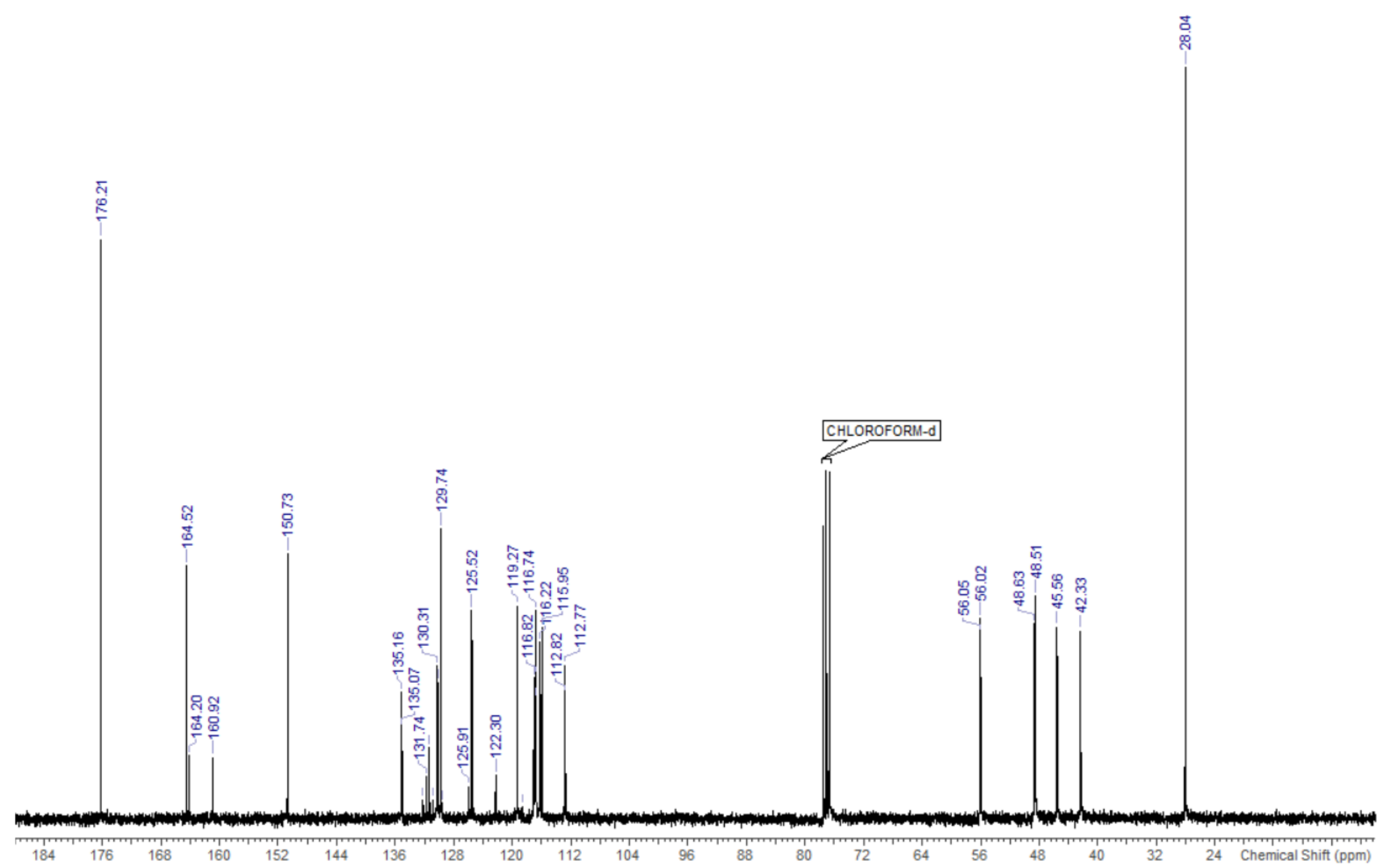


1-(1-(4-Fluorophenyl)-2-oxo-2-(4-(3-(trifluoromethyl)phenyl)piperazin-1-yl)ethyl)pyrrolidine-2,5-dione (35) - ${ }^{1} \mathrm{H}$ NMR

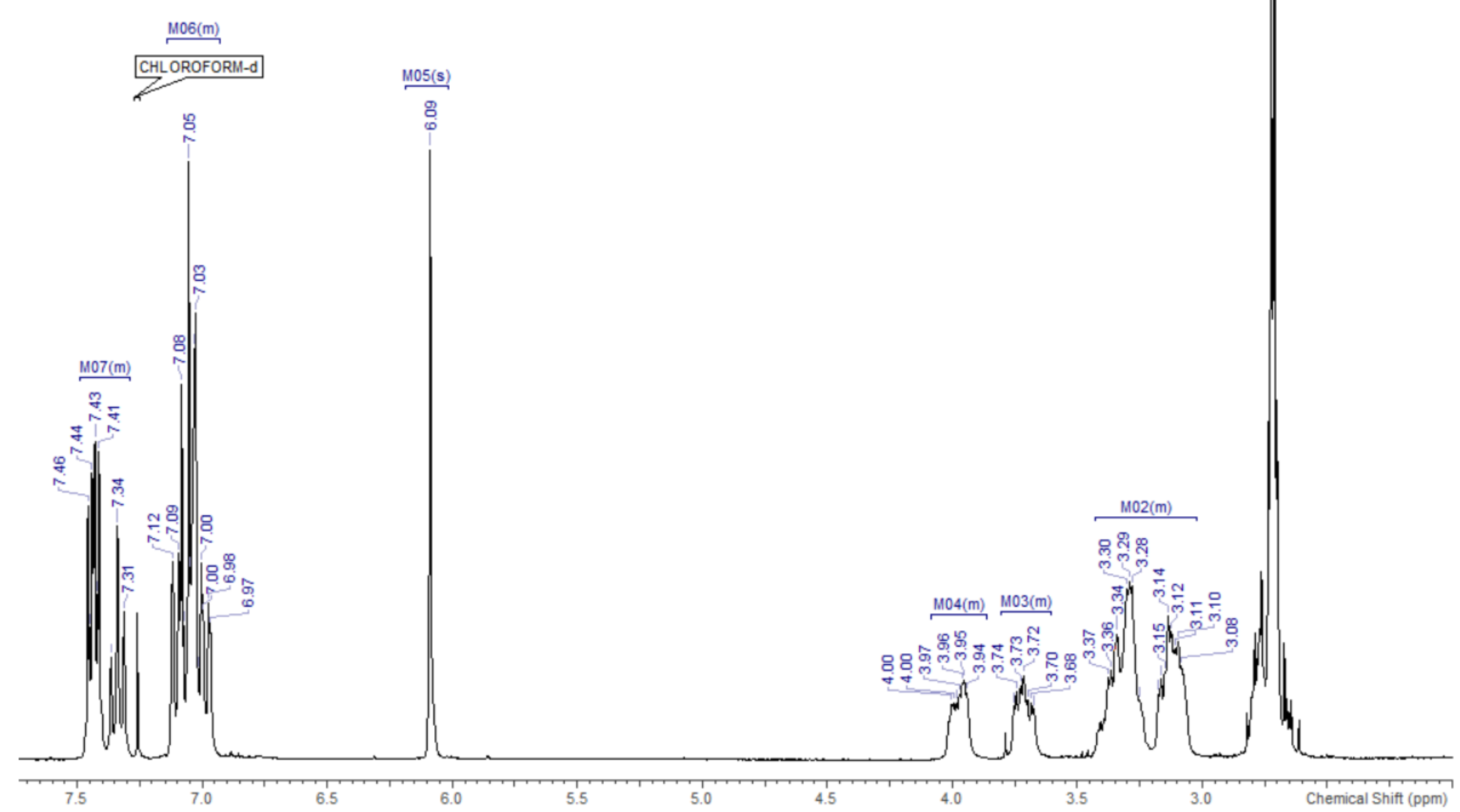


1-(1-(4-Fluorophenyl)-2-oxo-2-(4-(3-(trifluoromethyl)phenyl)piperazin-1-yl)ethyl)pyrrolidine-2,5-dione (35) - ${ }^{13} \mathrm{C} N \mathrm{NR}$

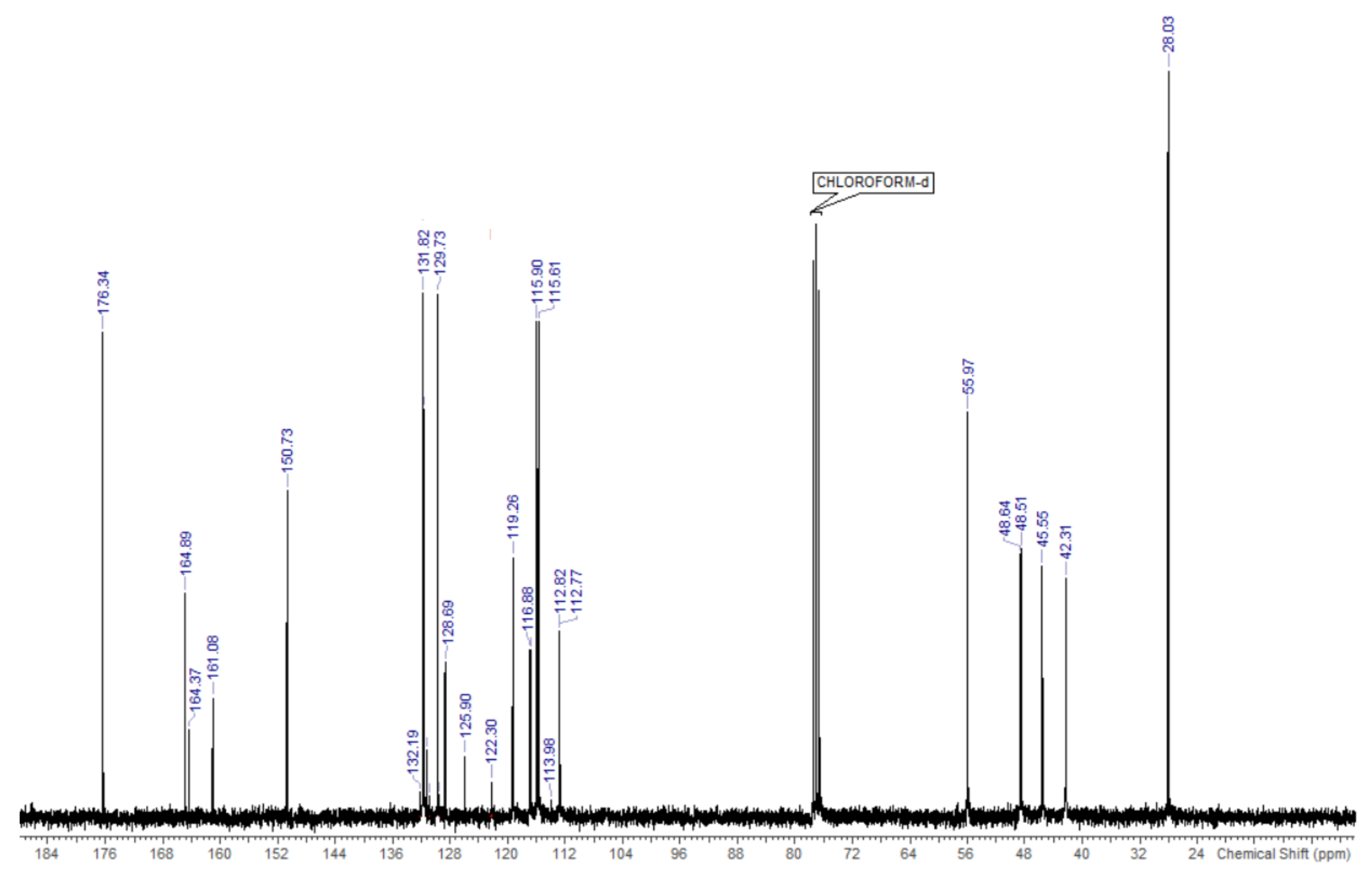


1-(1-(3,4-Difluorophenyl)-2-oxo-2-(4-(3-(trifluoromethyl)phenyl)piperazin-1-yl)ethyl)pyrrolidine-2,5-dione (36) - ${ }^{1} \mathrm{H}$ NMR

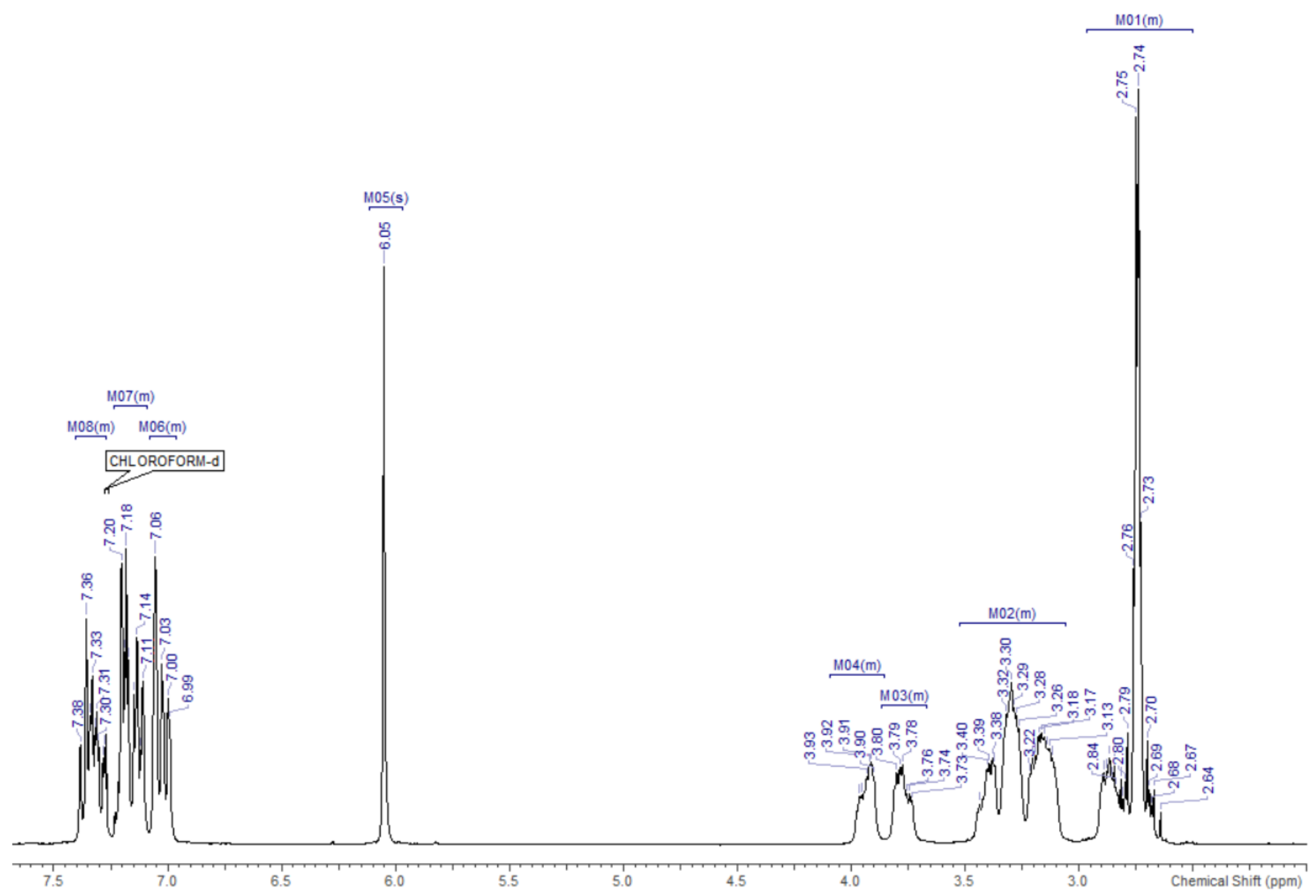


1-(1-(3,4-Difluorophenyl)-2-oxo-2-(4-(3-(trifluoromethyl)phenyl)piperazin-1-yl)ethyl)pyrrolidine-2,5-dione (36) - ${ }^{13} \mathrm{C}$ NMR

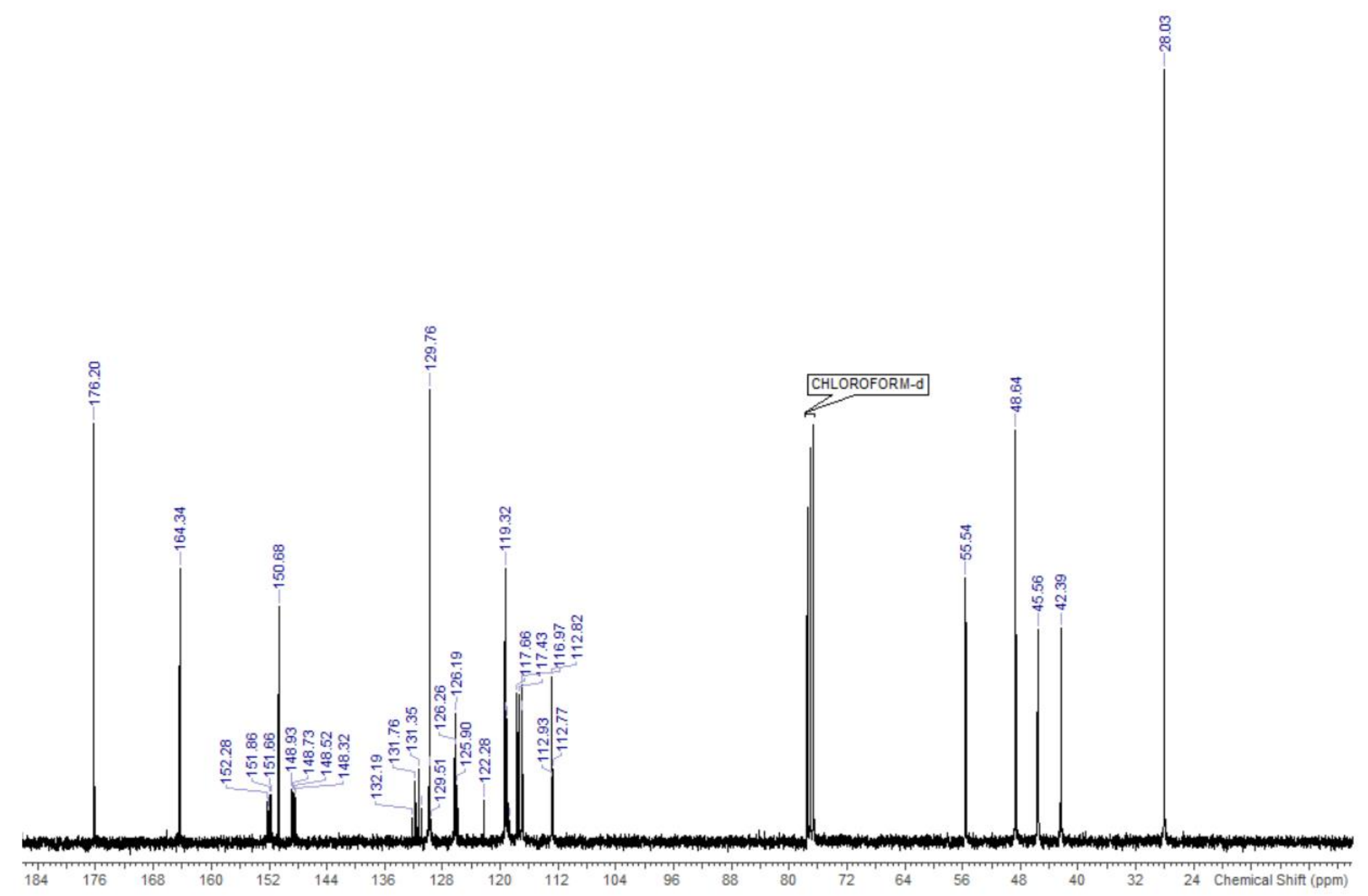


1-(1-Oxo-3-phenyl-1-(4-phenylpiperazin-1-yl)propan-2-yl)pyrrolidine-2,5-dione (37) - ${ }^{1} \mathrm{H}$ NMR

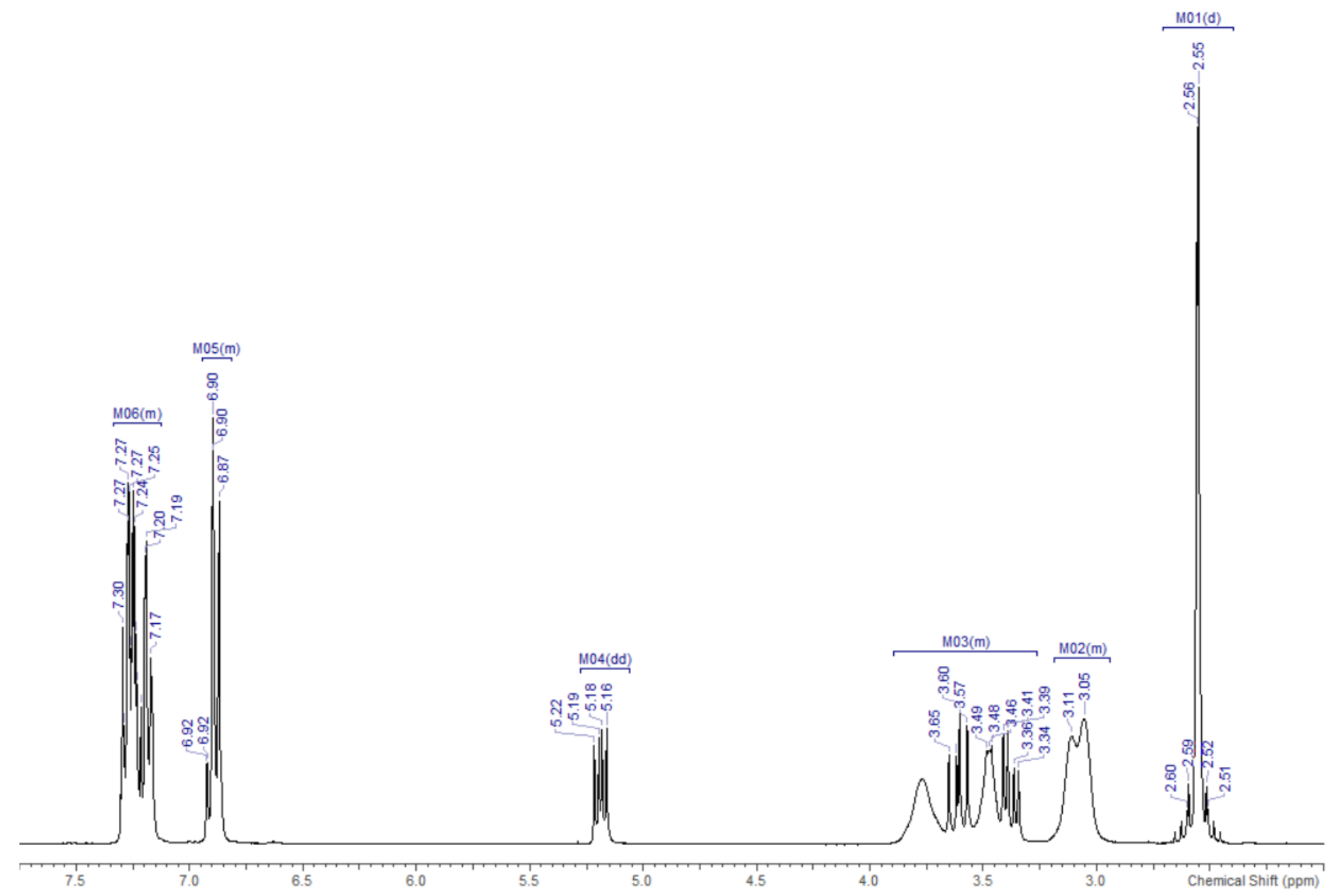


1-(1-Oxo-3-phenyl-1-(4-phenylpiperazin-1-yl)propan-2-yl)pyrrolidine-2,5-dione (37) - ${ }^{13} \mathrm{C}$ NMR

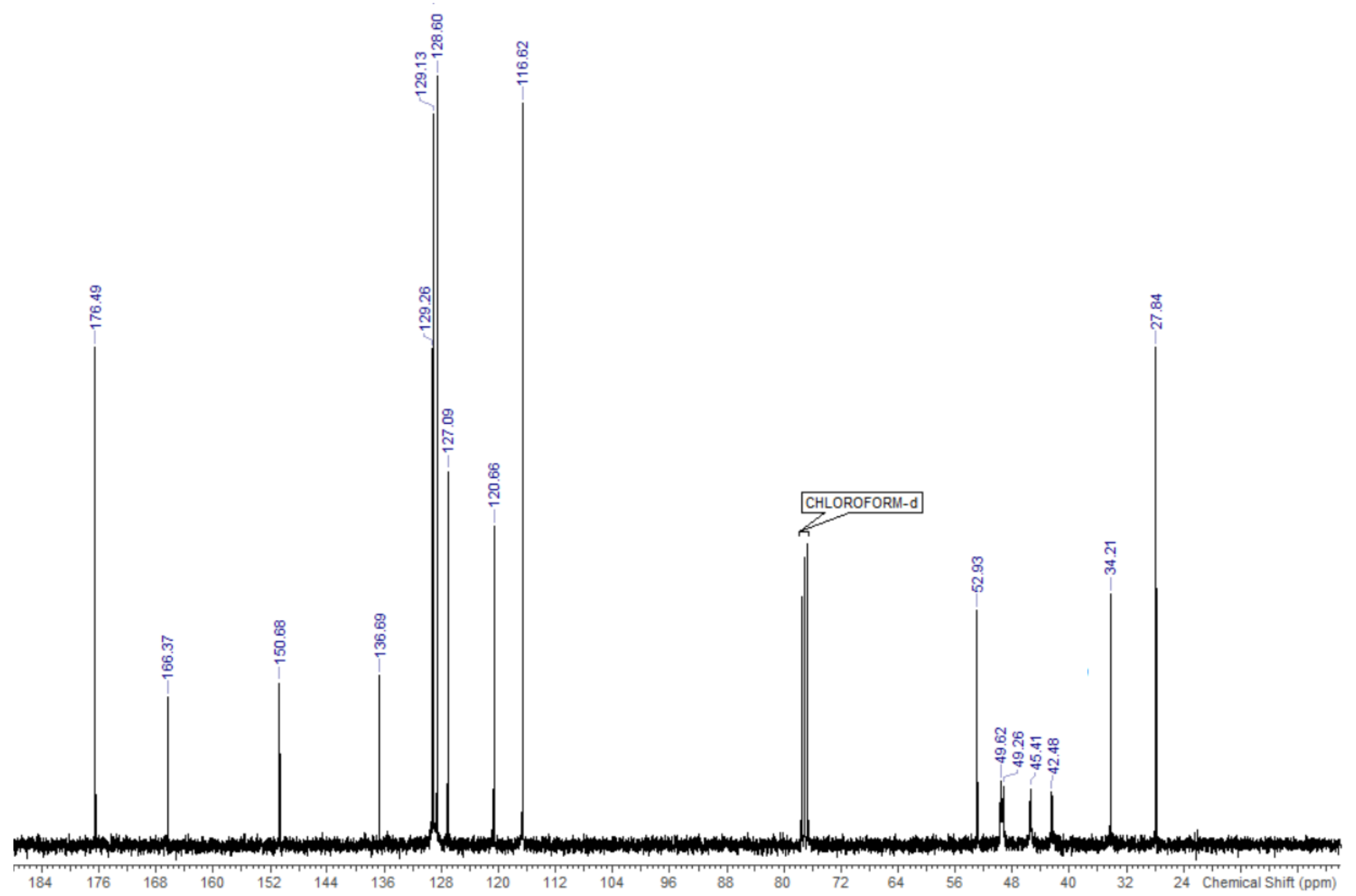


1-(1-(4-(3-Chlorophenyl)piperazin-1-yl)-1-oxo-3-phenylpropan-2-yl)pyrrolidine-2,5-dione (38) - ${ }^{1} \mathrm{H}$ NMR

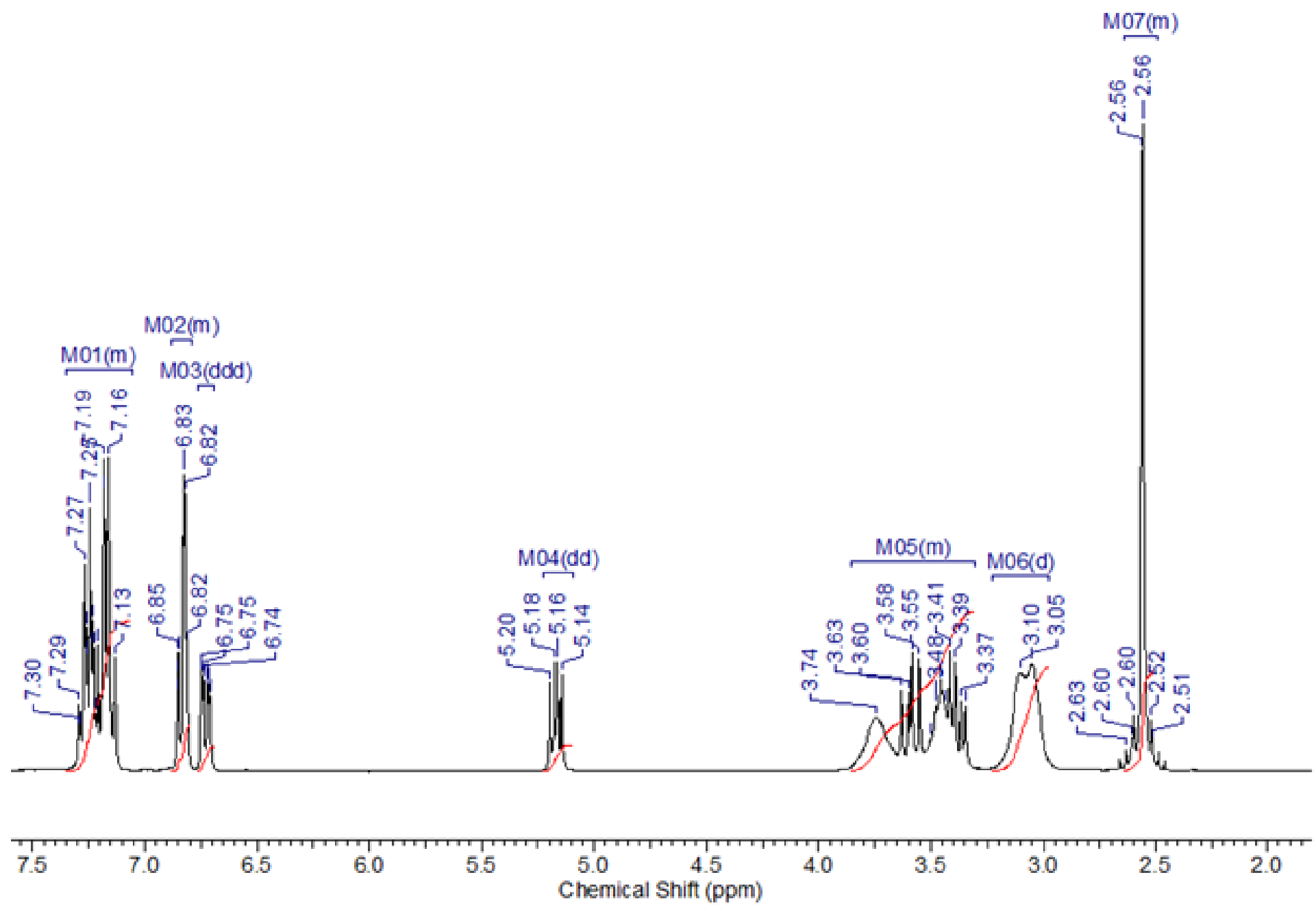




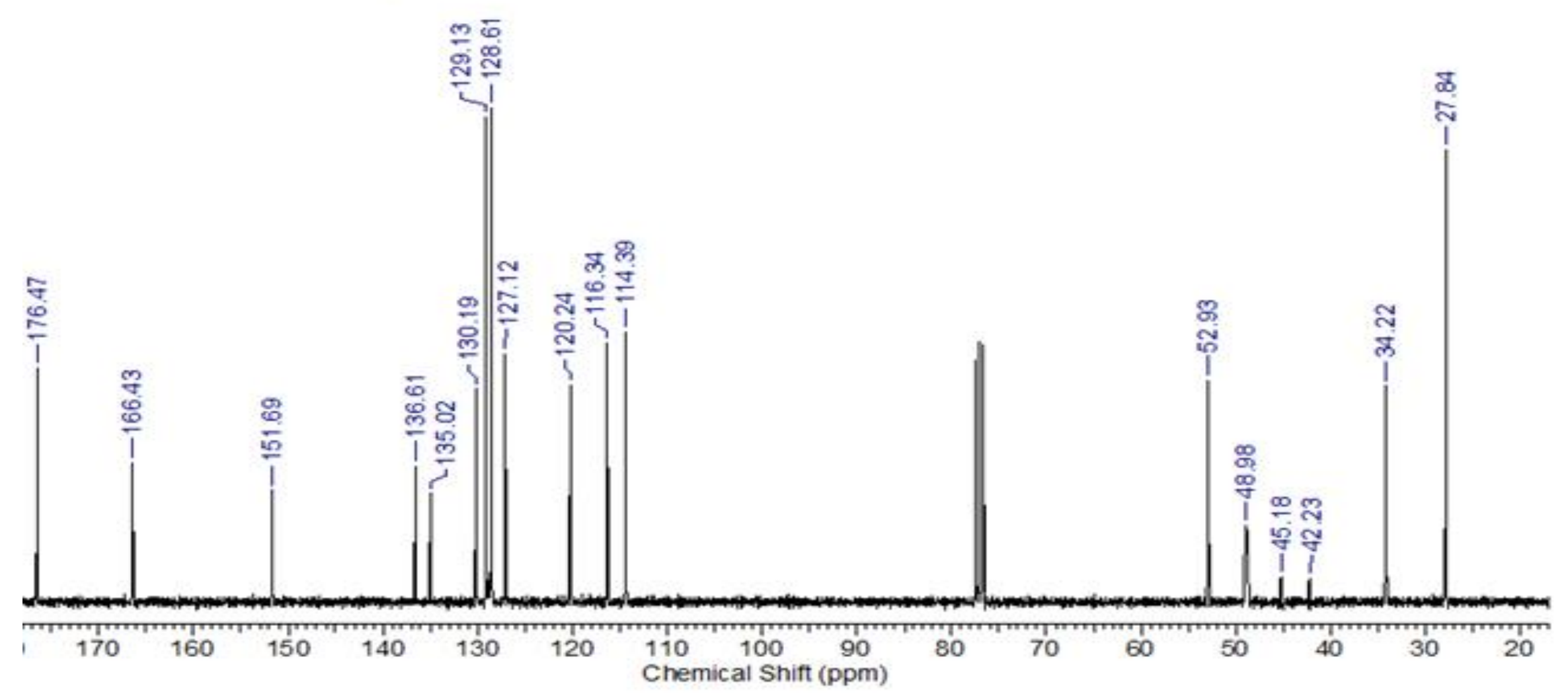


1-(1-Oxo-3-phenyl-1-(4-(m-tolyl)piperazin-1-yl)propan-2-yl)pyrrolidine-2,5-dione (39) - ${ }^{1} \mathrm{H}$ NMR

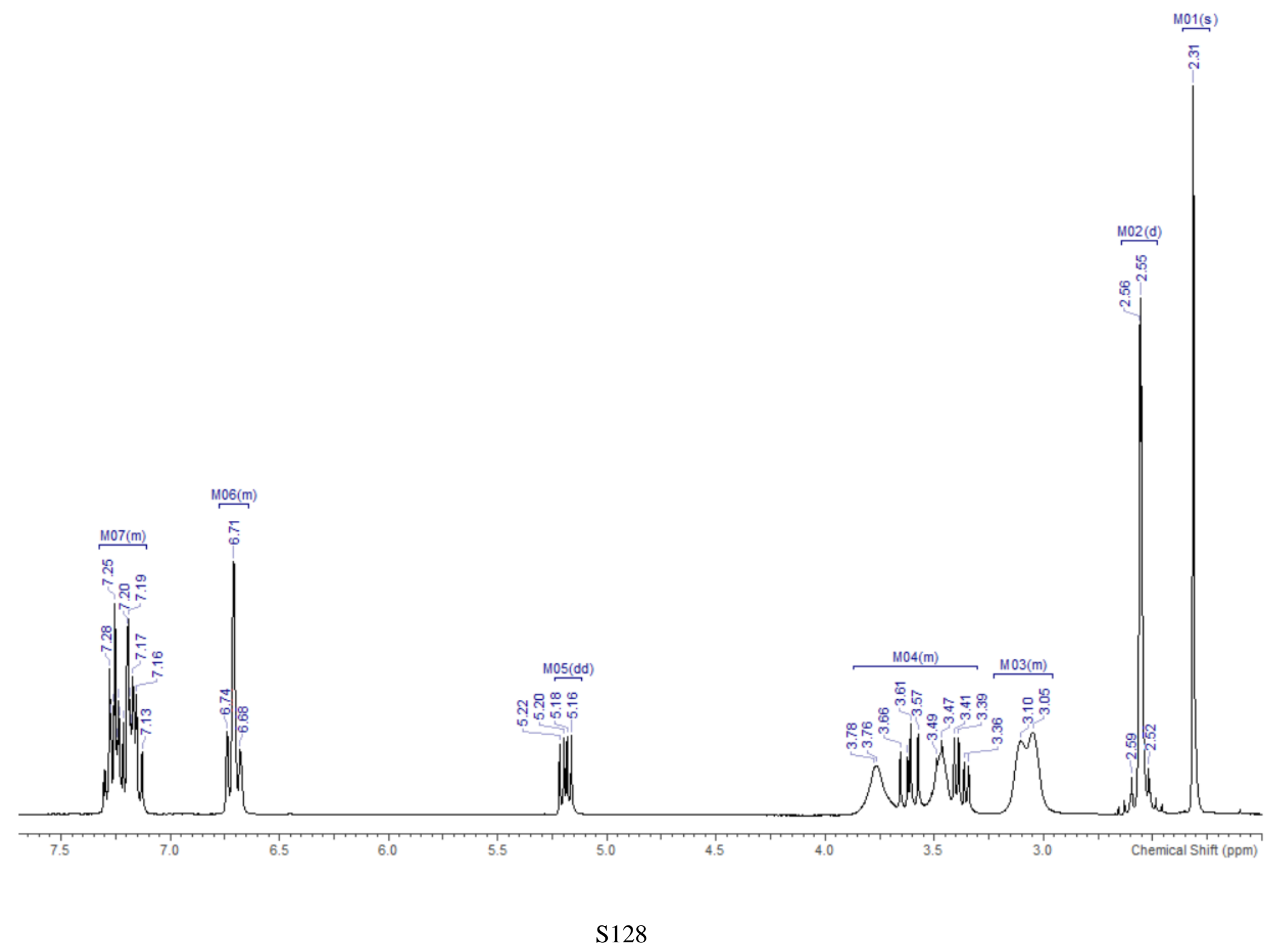


1-(1-Oxo-3-phenyl-1-(4-(m-tolyl)piperazin-1-yl)propan-2-yl)pyrrolidine-2,5-dione (39) - ${ }^{13} \mathrm{C}$ NMR

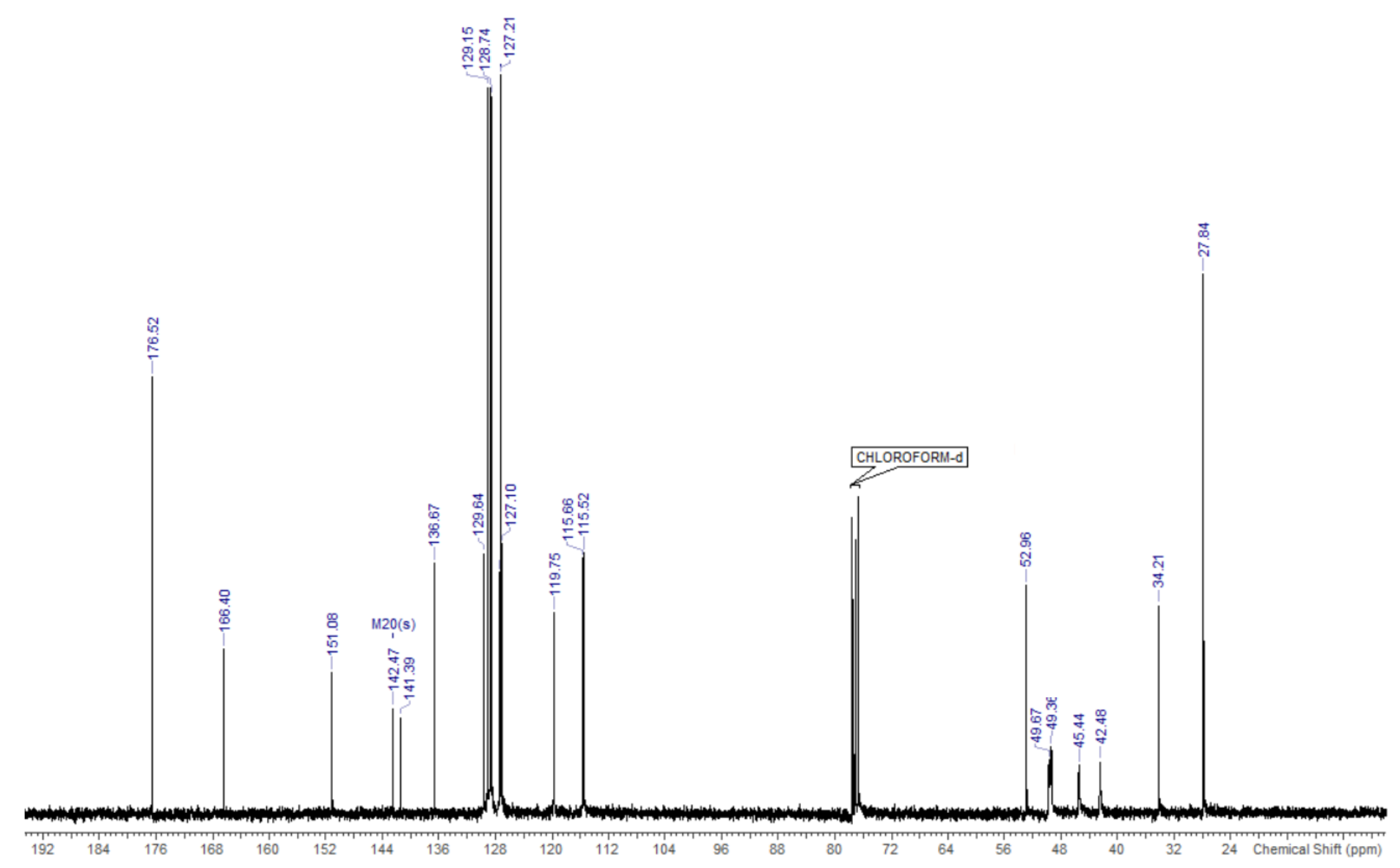


1-(1-Oxo-3-phenyl-1-(4-(3-(trifluoromethyl)phenyl)piperazin-1-yl)propan-2-yl)pyrrolidine-2,5-dione (40) - ${ }^{1} \mathrm{H} N \mathrm{NR}$

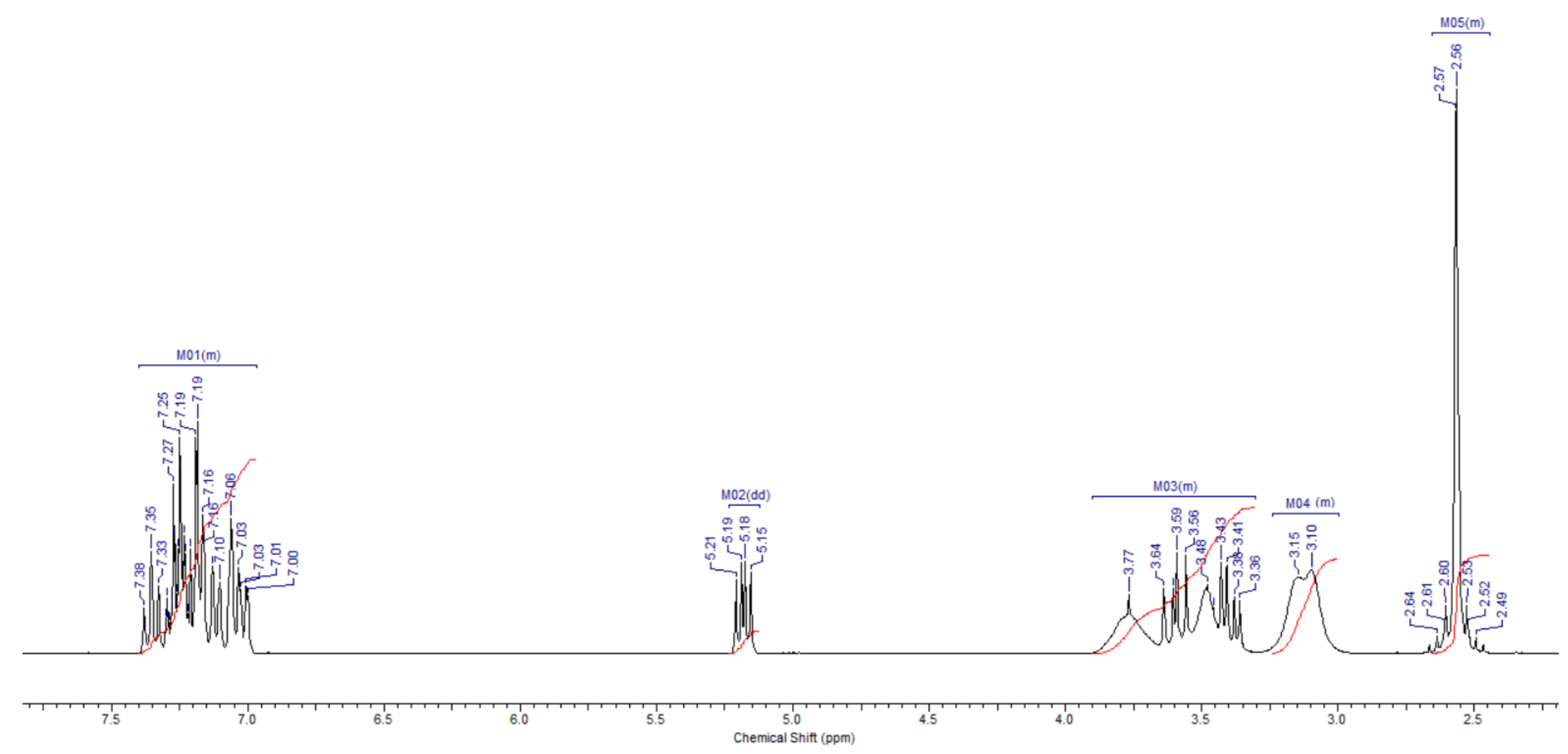


1-(1-Oxo-3-phenyl-1-(4-(3-(trifluoromethyl)phenyl)piperazin-1-yl)propan-2-yl)pyrrolidine-2,5-dione (40) - ${ }^{13} \mathrm{C} \mathrm{NMR}$

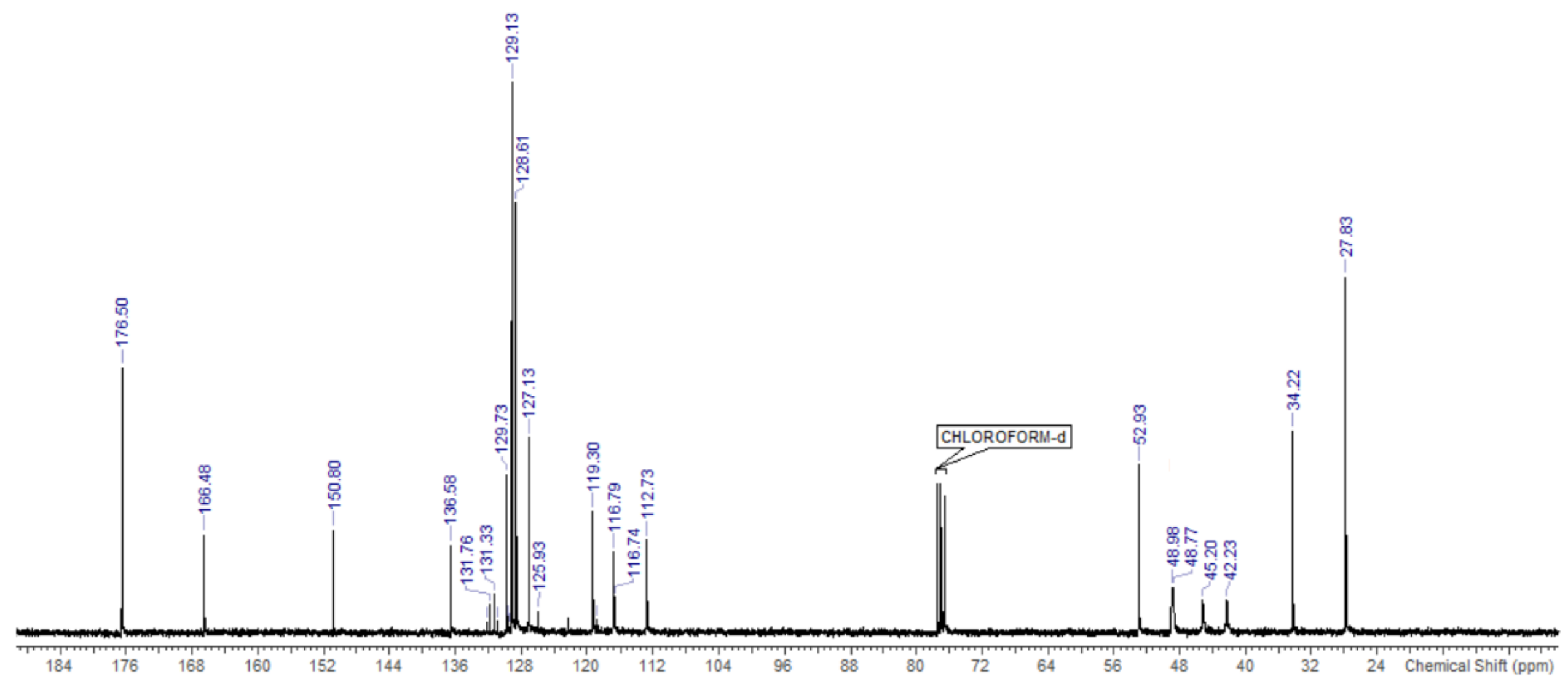


1-(1-Oxo-3-phenyl-1-(4-(3-(trifluoromethyl)phenyl)piperazin-1-yl)propan-2-yl)pyrrolidine-2,5-dione (40) - ${ }^{19} \mathrm{~F}$ NMR

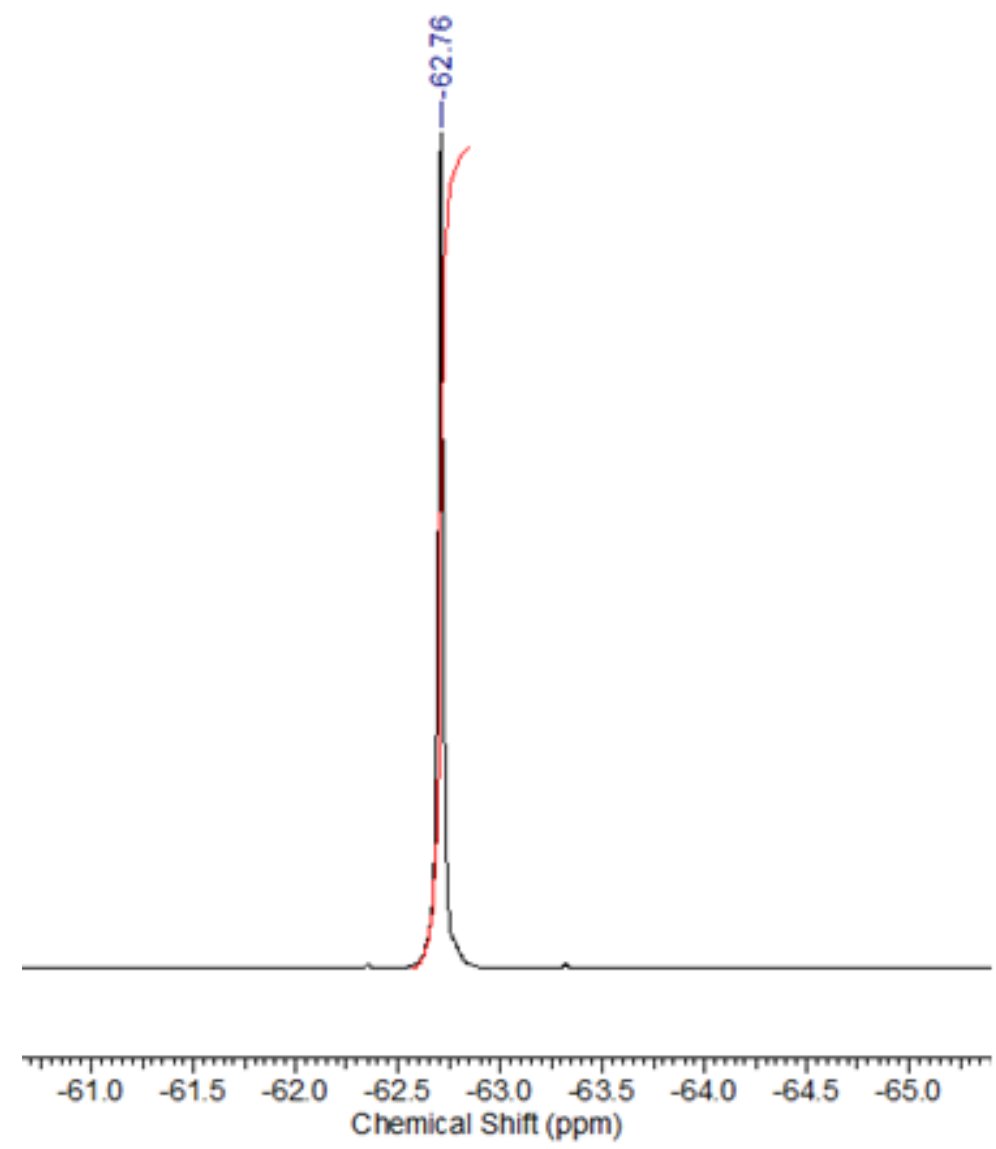


1-(1-Oxo-3-phenyl-1-(4-(3-(trifluoromethoxy)phenyl)piperazin-1-yl)propan-2-yl)pyrrolidine-2,5-dione (41) - ${ }^{1} \mathrm{H}$ NMR

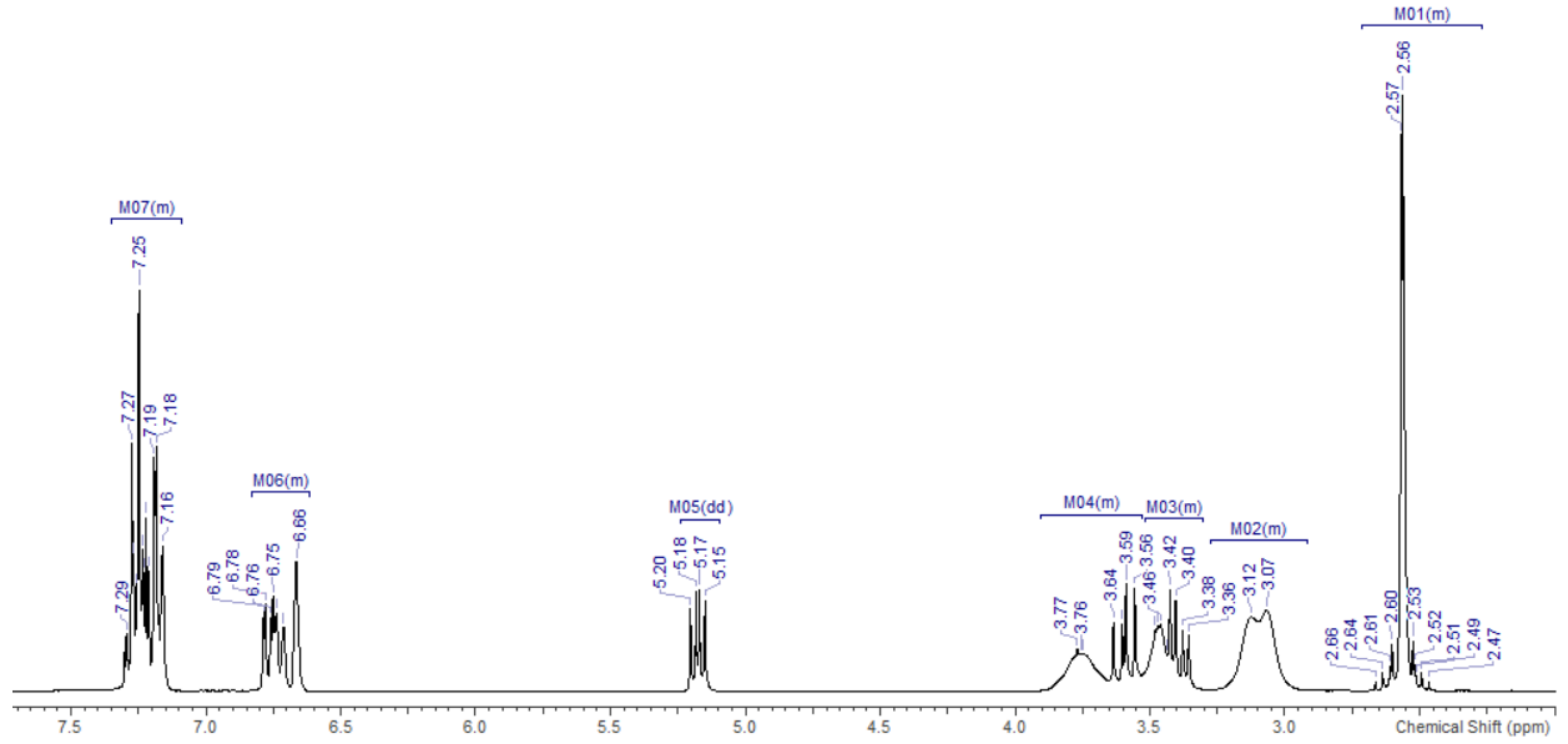


1-(1-Oxo-3-phenyl-1-(4-(3-(trifluoromethoxy)phenyl)piperazin-1-yl)propan-2-yl)pyrrolidine-2,5-dione (41) - ${ }^{13} \mathrm{C} \mathrm{NMR}$

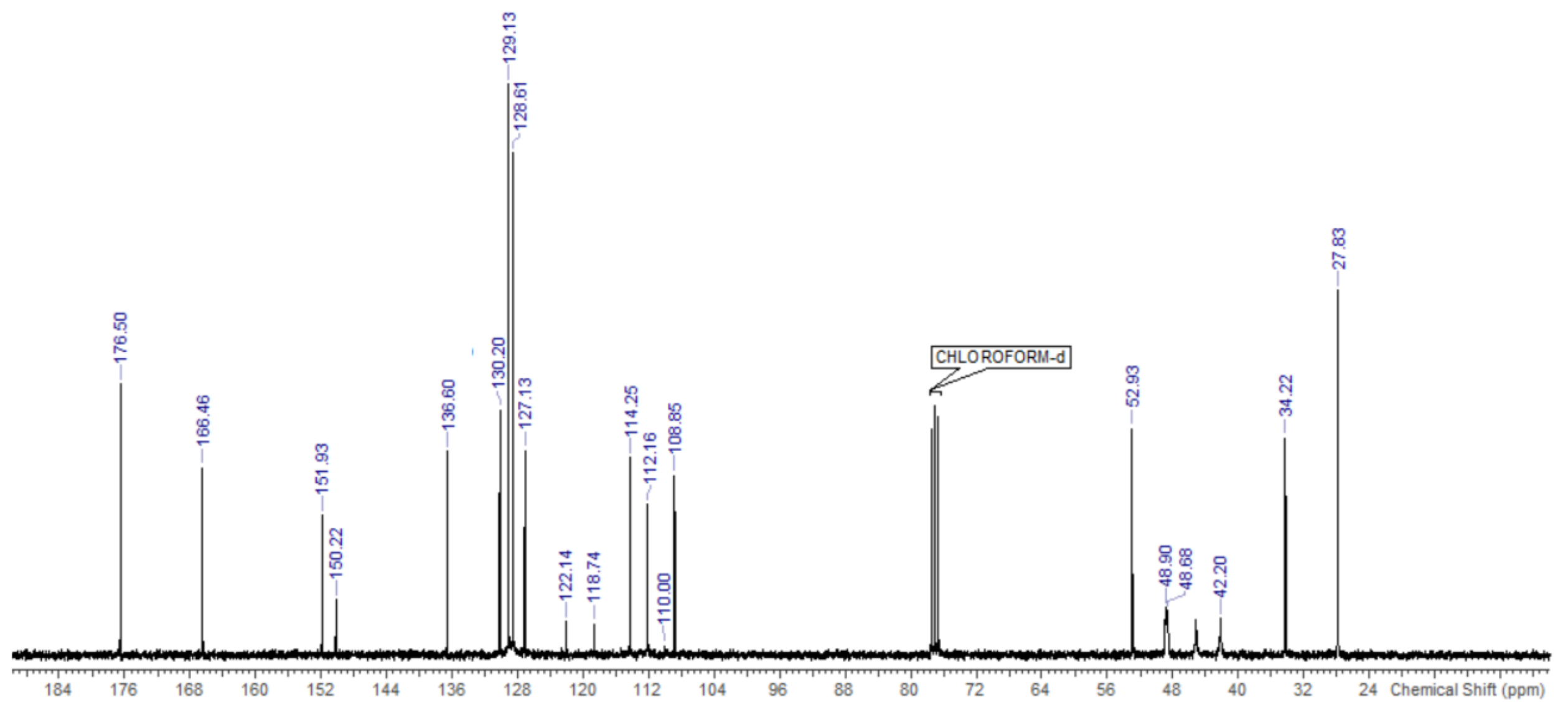


1-(2-(4-(2,3-Dichlorophenyl)piperazin-1-yl)-2-oxo-1-phenylethyl)pyrrolidine-2,5-dione (42) - ${ }^{1} \mathrm{H}$ NMR

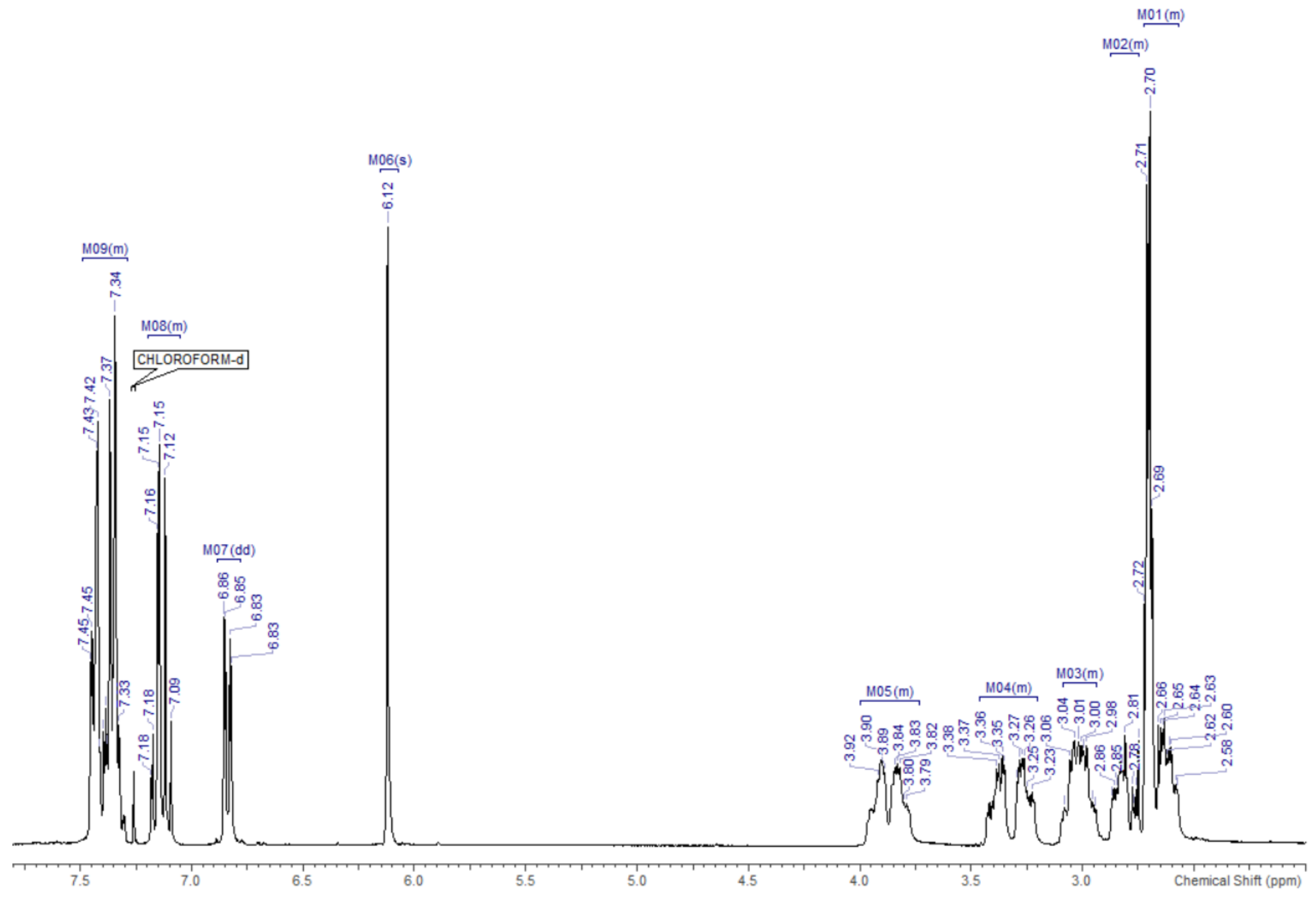


1-(2-(4-(2,3-Dichlorophenyl)piperazin-1-yl)-2-oxo-1-phenylethyl)pyrrolidine-2,5-dione (42) - ${ }^{13} \mathrm{C}$ NMR

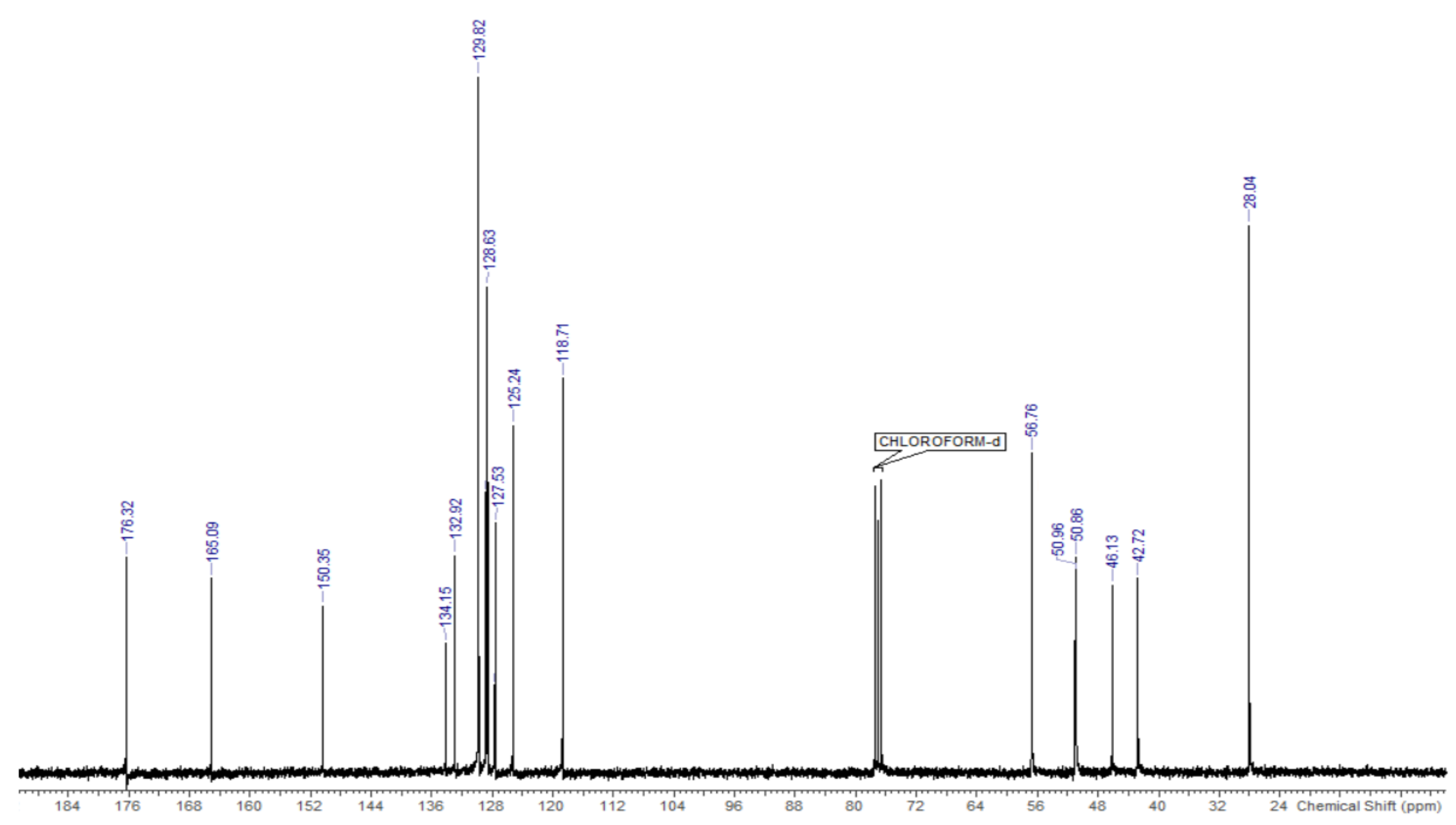


1-(2-(4-(3,4-Dichlorophenyl)piperazin-1-yl)-2-oxo-1-phenylethyl)pyrrolidine-2,5-dione (43) - ${ }^{1} \mathrm{H}$ NMR

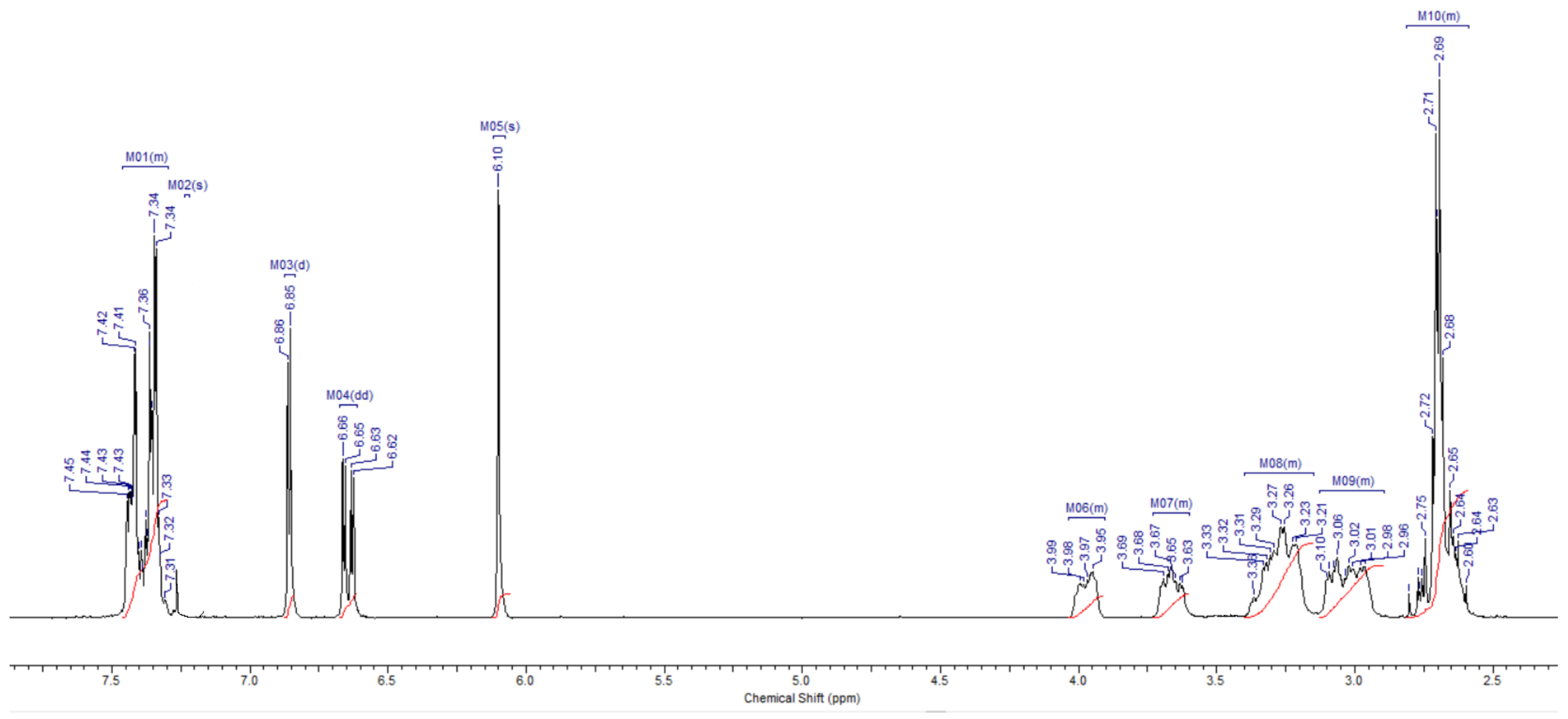


1-(2-(4-(3,4-Dichlorophenyl)piperazin-1-yl)-2-oxo-1-phenylethyl)pyrrolidine-2,5-dione (43) - ${ }^{13} \mathrm{C}$ NMR

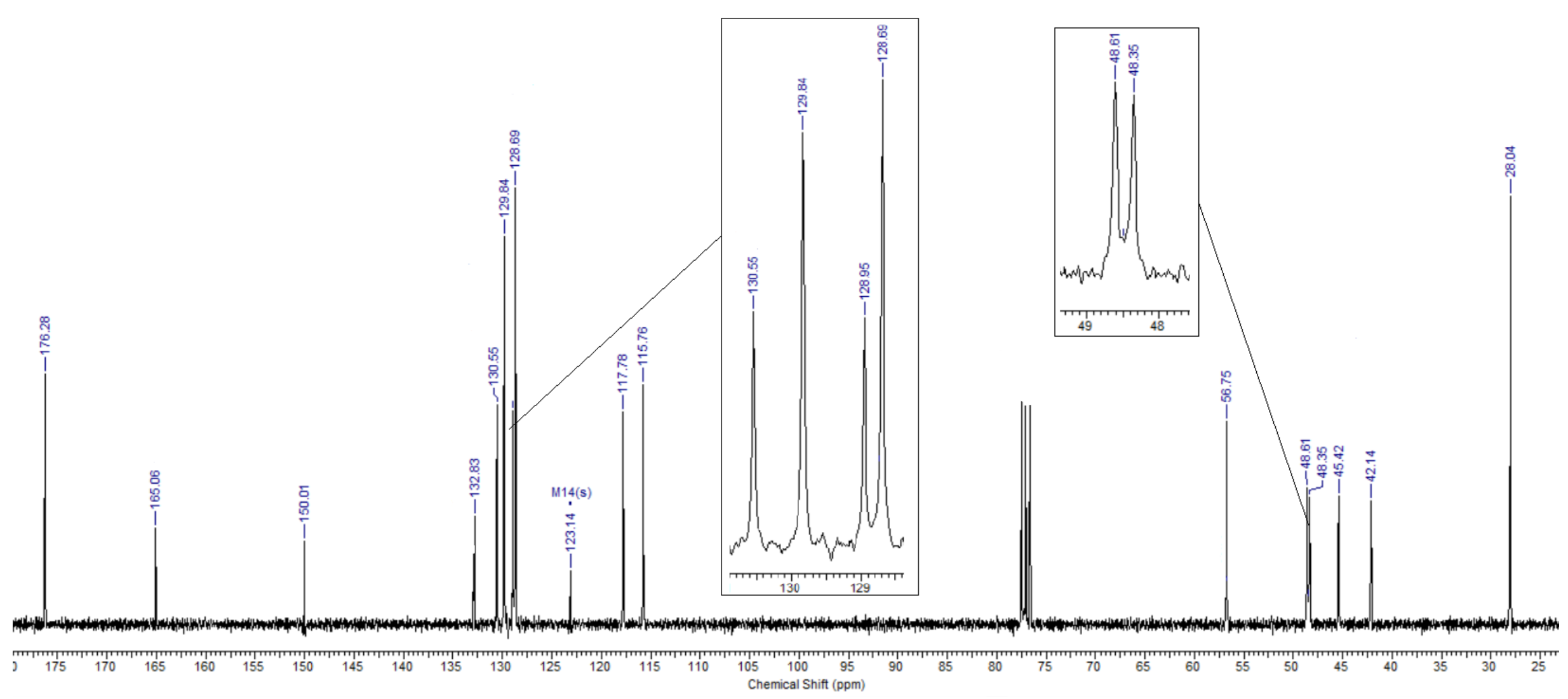


1-(2-(4-(3,5-Dichlorophenyl)piperazin-1-yl)-2-oxo-1-phenylethyl)pyrrolidine-2,5-dione (44) - ${ }^{1} \mathrm{H}$ NMR

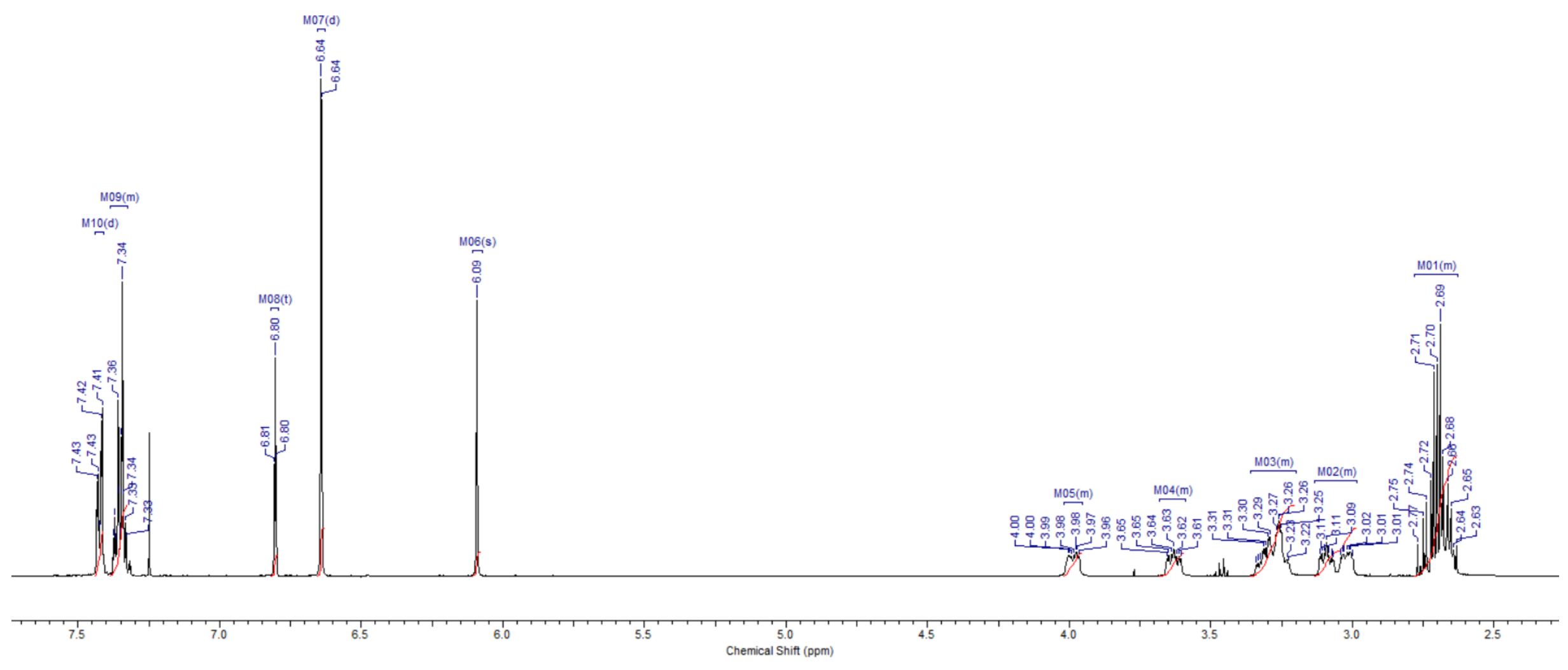


1-(2-(4-(3,5-Dichlorophenyl)piperazin-1-yl)-2-oxo-1-phenylethyl)pyrrolidine-2,5-dione (44) - ${ }^{13} \mathrm{C}$ NMR

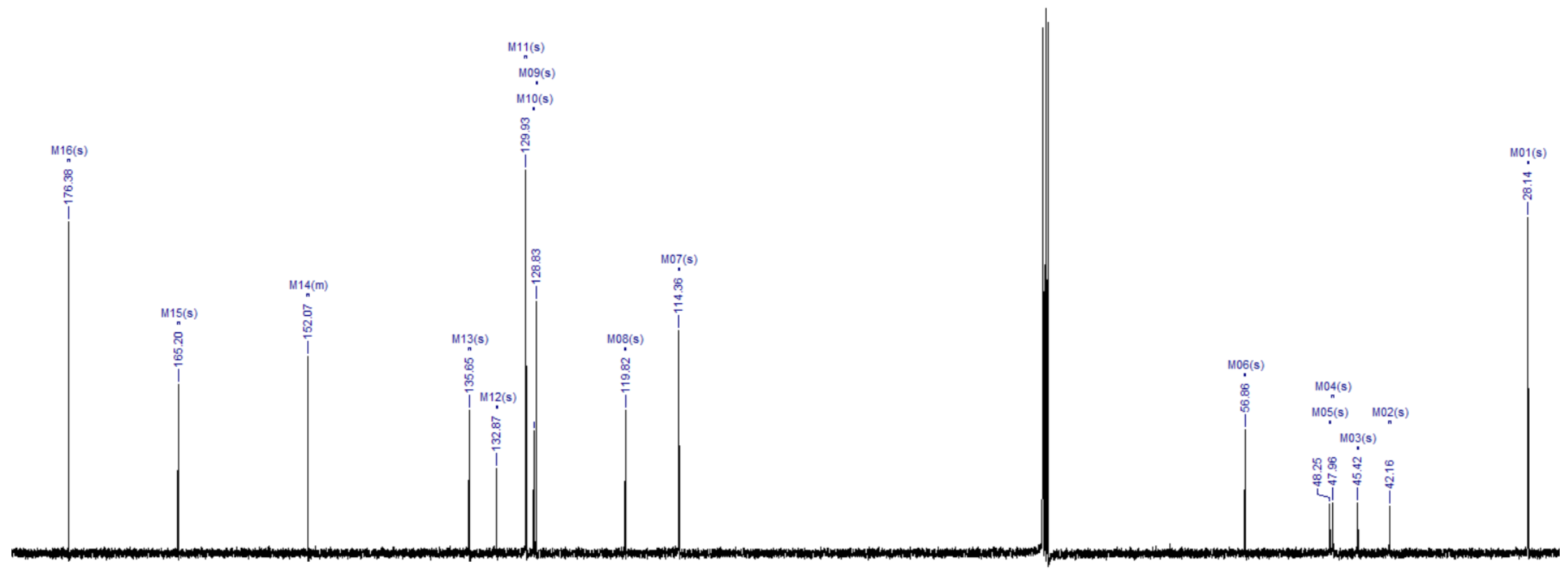

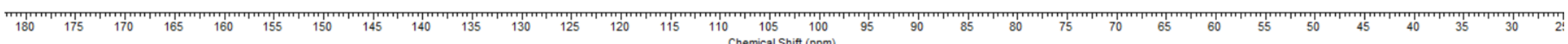


1-(2-(4-(3,5-bis(trifluoromethyl)phenyl)piperazin-1-yl)-2-oxo-1-phenylethyl)pyrrolidine-2,5-dione (45) - ${ }^{1} \mathrm{H}$ NMR

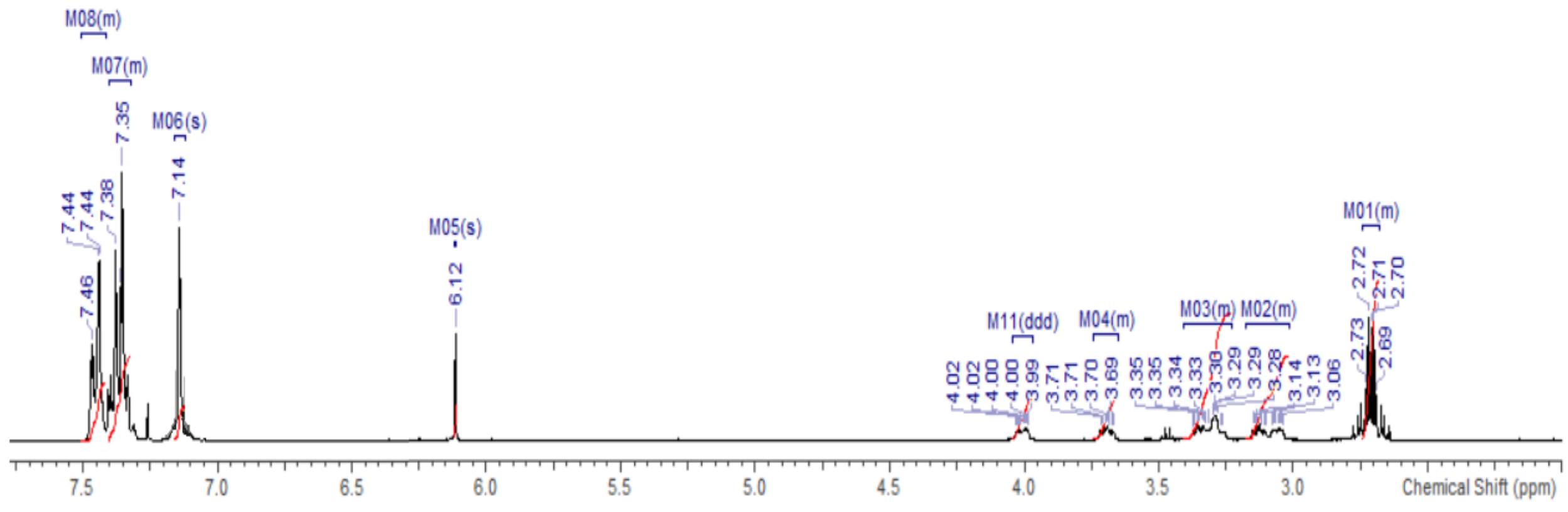


1-(2-(4-(3,5-bis((trifluoromethyl)thio)phenyl)piperazin-1-yl)-2-oxo-1-phenylethyl)pyrrolidine-2,5-dione (46) - ${ }^{1} \mathrm{H}$ NMR

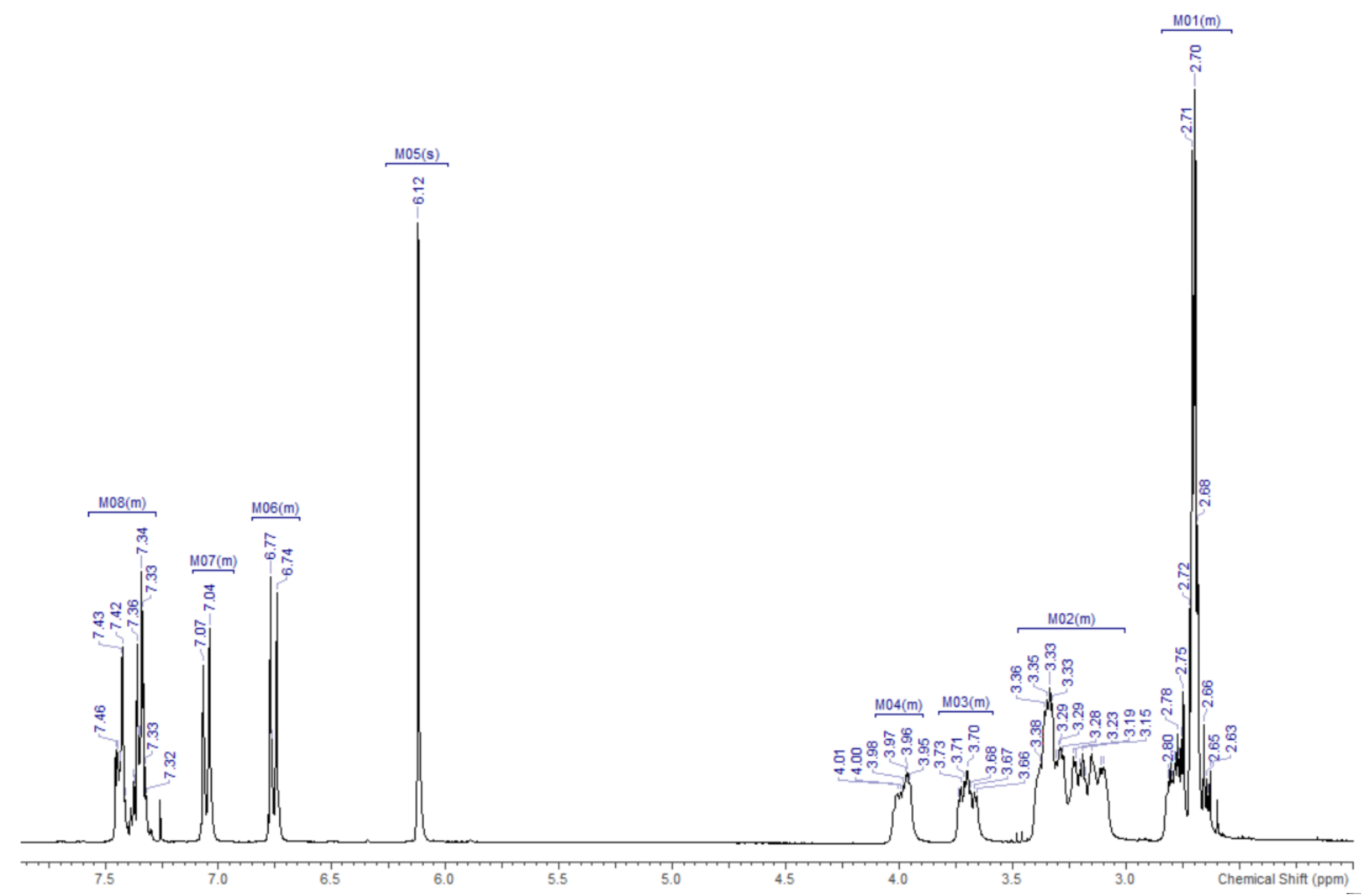


1-(2-(4-(3-chloro-5-(trifluoromethyl)phenyl)piperazin-1-yl)-2-oxo-1-phenylethyl)pyrrolidine-2,5-dione (47) - ${ }^{1} \mathrm{H}$ NMR

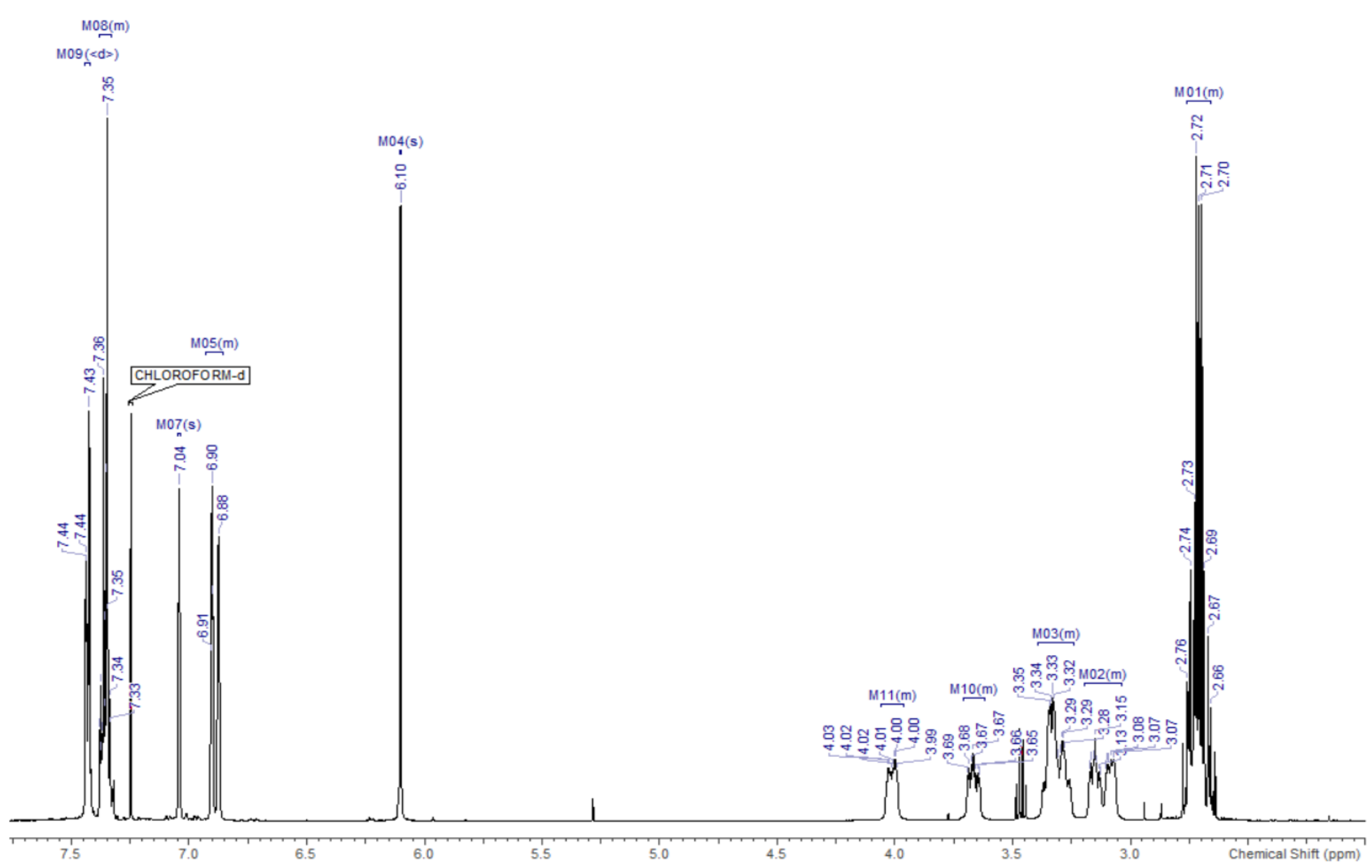


1-(2-(4-(3-chloro-5-(trifluoromethyl)phenyl)piperazin-1-yl)-2-oxo-1-phenylethyl)pyrrolidine-2,5-dione (47) - ${ }^{13} \mathrm{C} N \mathrm{NR}$

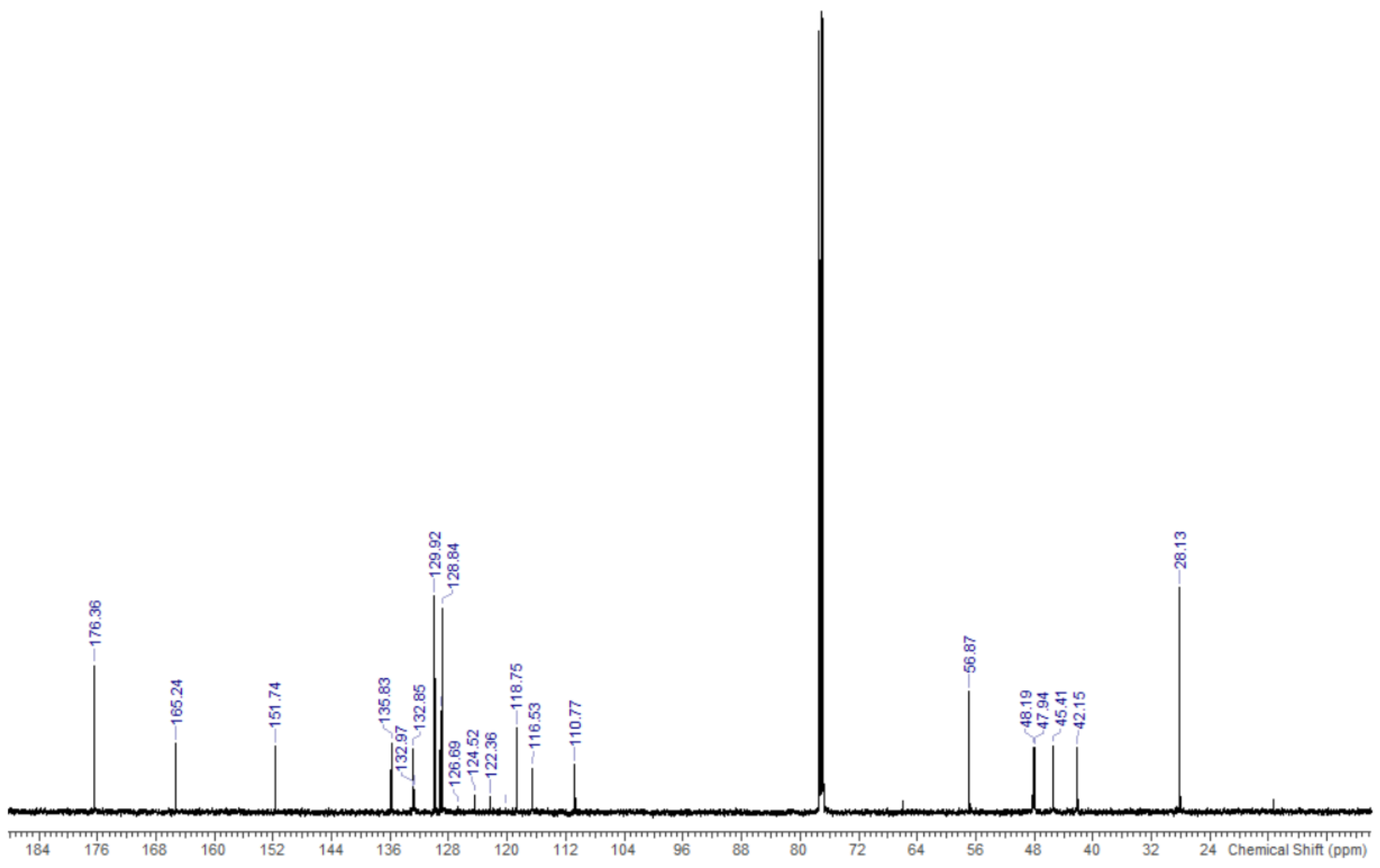

S144 
1-(2-Oxo-1-phenyl-2-(4-(3-(trifluoromethyl)phenyl)piperidin-1-yl)ethyl)pyrrolidine-2,5-dione (48) - ${ }^{1} \mathrm{H}$ NMR

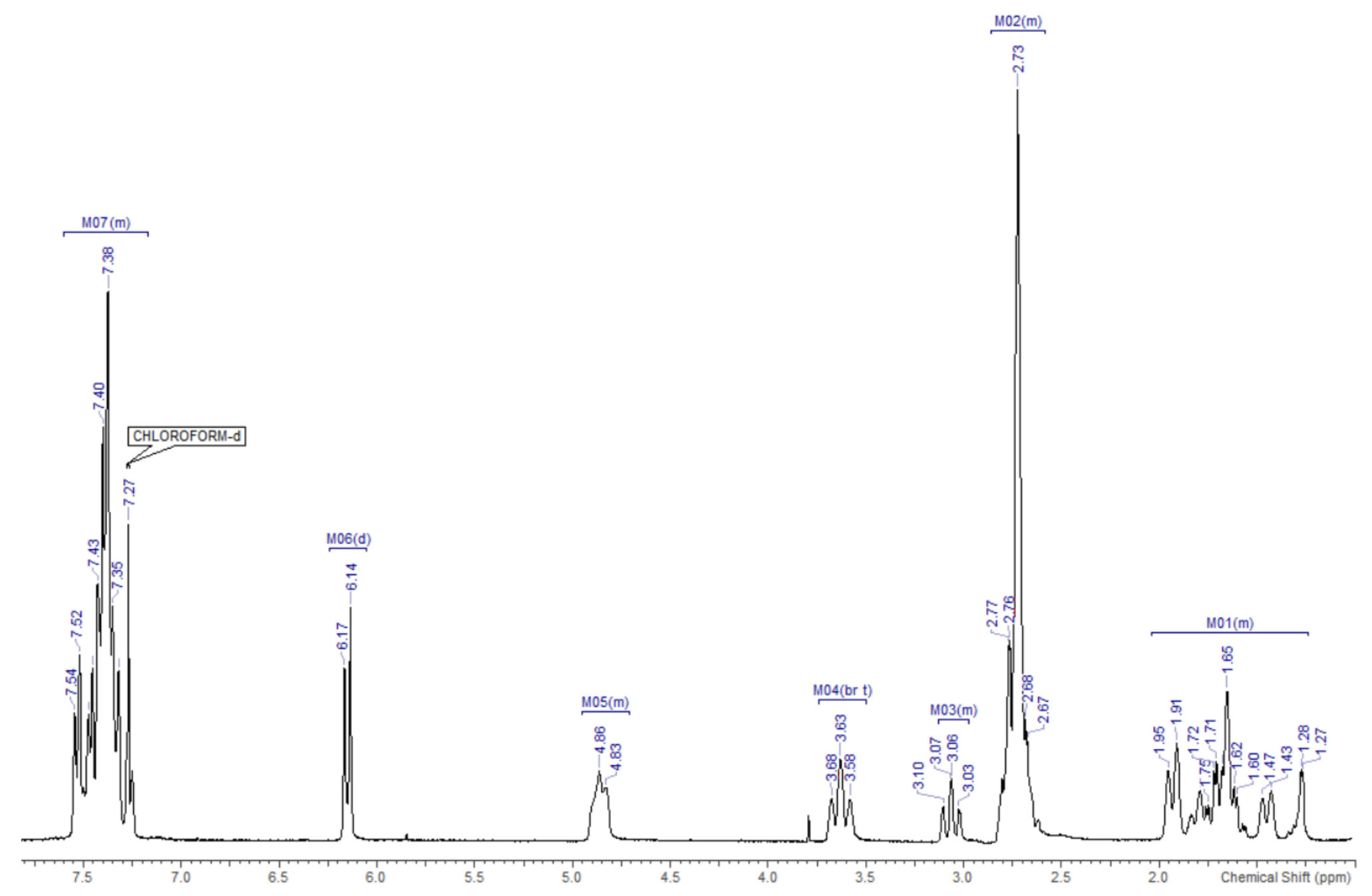


1-(2-Oxo-1-phenyl-2-(4-(3-(trifluoromethyl)phenyl)-1,4-diazepan-1-yl)ethyl)pyrrolidine-2,5-dione (49) - ${ }^{1} \mathrm{H}$ NMR

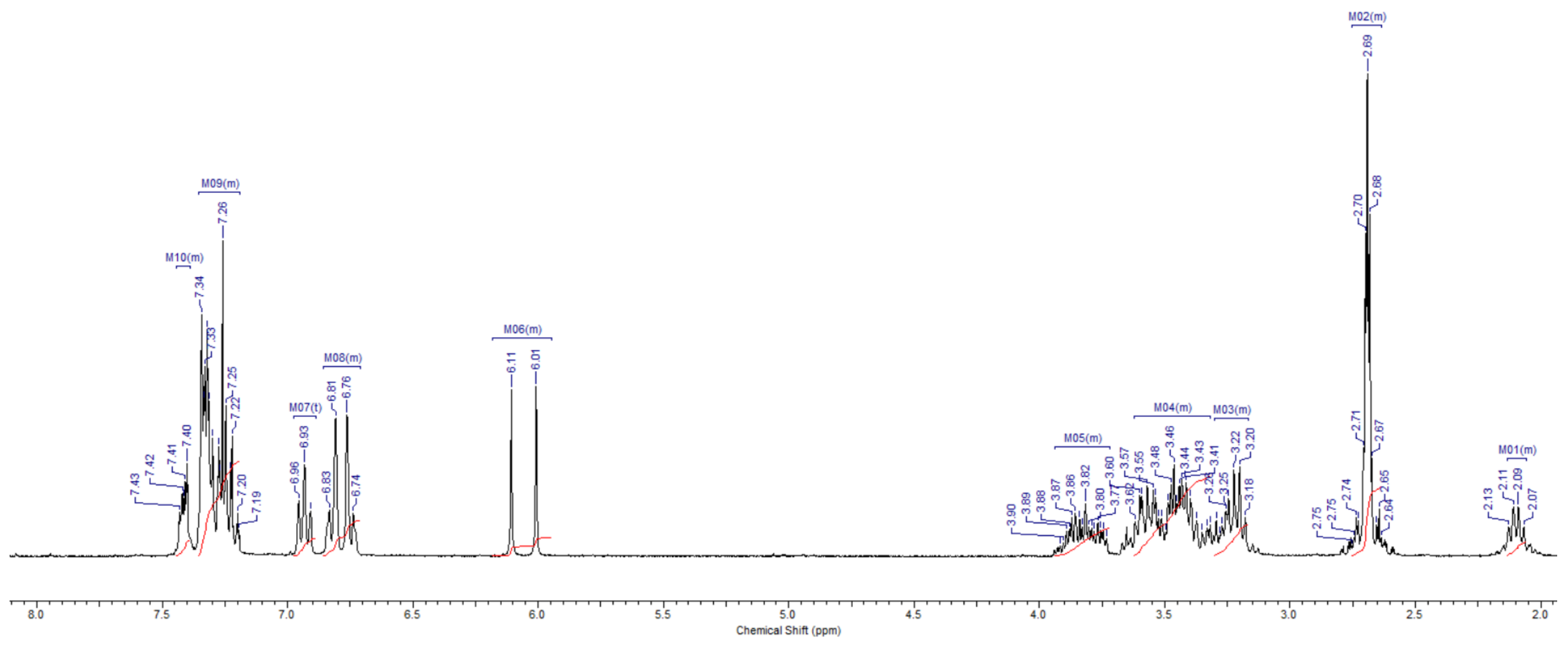


1-(3-(4-(3-(Trifluoromethyl)phenyl)piperazine-1-carbonyl)phenyl)pyrrolidine-2,5-dione (52)- ${ }^{1} \mathrm{H}$ NMR

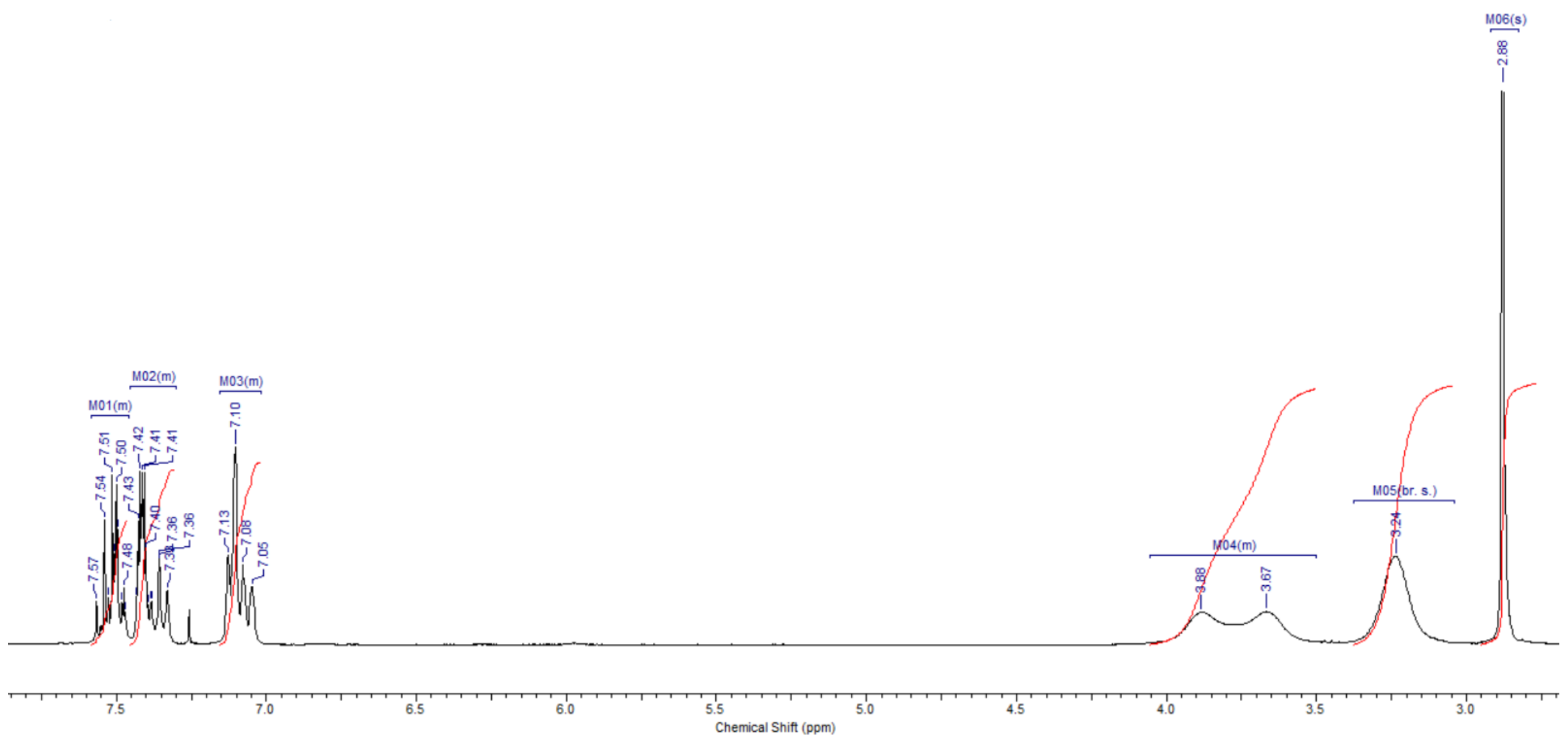


1-(3-(4-(3-(Trifluoromethyl)phenyl)piperazine-1-carbonyl)phenyl)pyrrolidine-2,5-dione (52)- ${ }^{19} \mathrm{~F}$ NMR

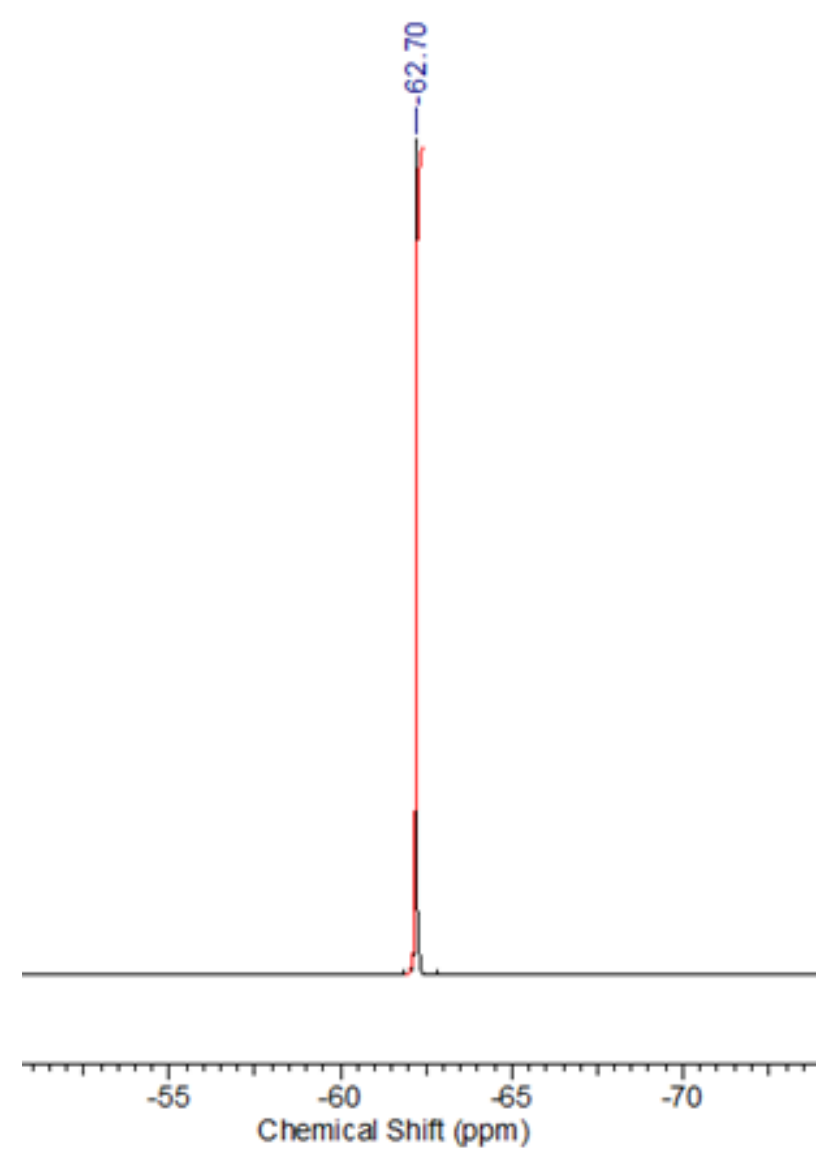


1-(4-(4-(3-(Trifluoromethyl)phenyl)piperazine-1-carbonyl)phenyl)pyrrolidine-2,5-dione (53) - ${ }^{1} \mathrm{H}$ NMR

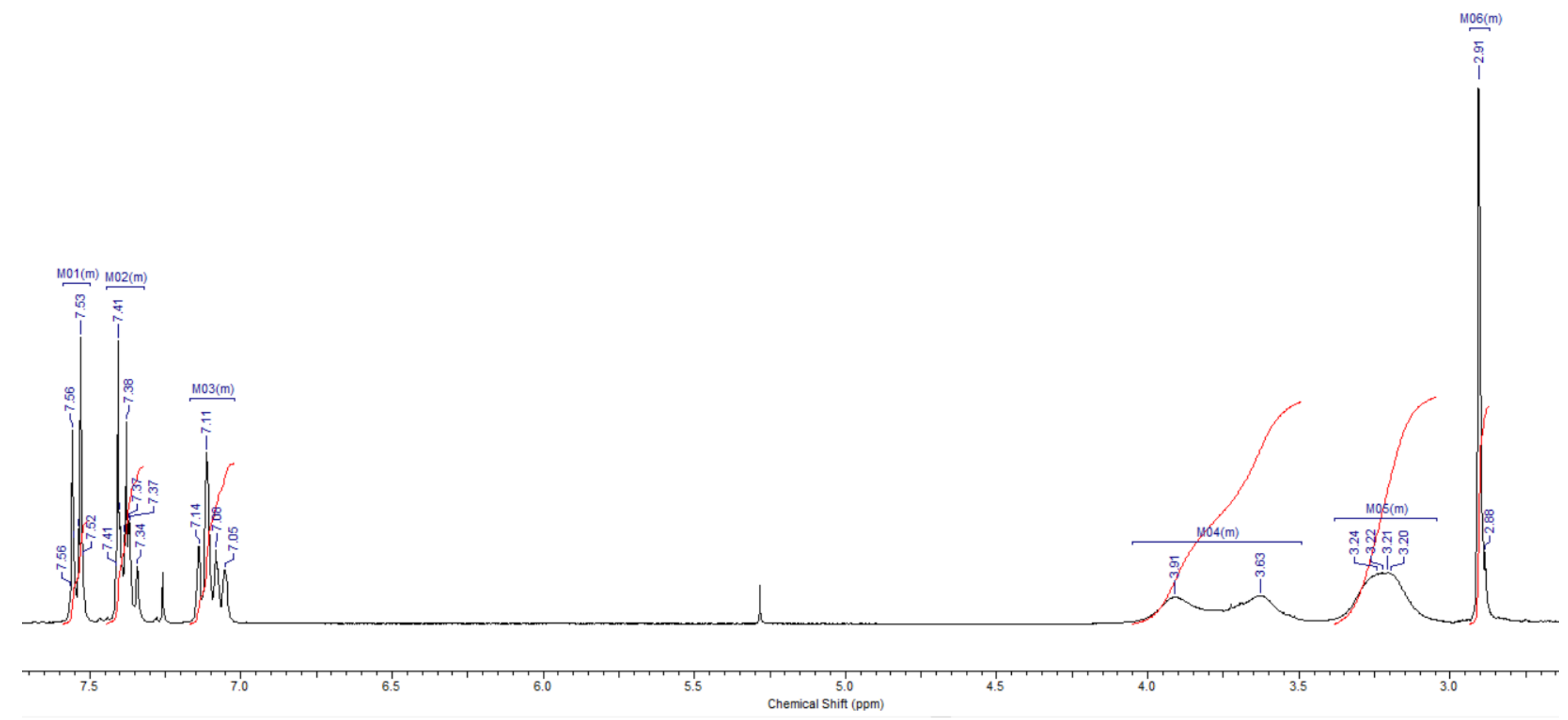


1-(4-(4-(3-(Trifluoromethyl)phenyl)piperazine-1-carbonyl)phenyl)pyrrolidine-2,5-dione (53) - ${ }^{13} \mathrm{C}$ NMR

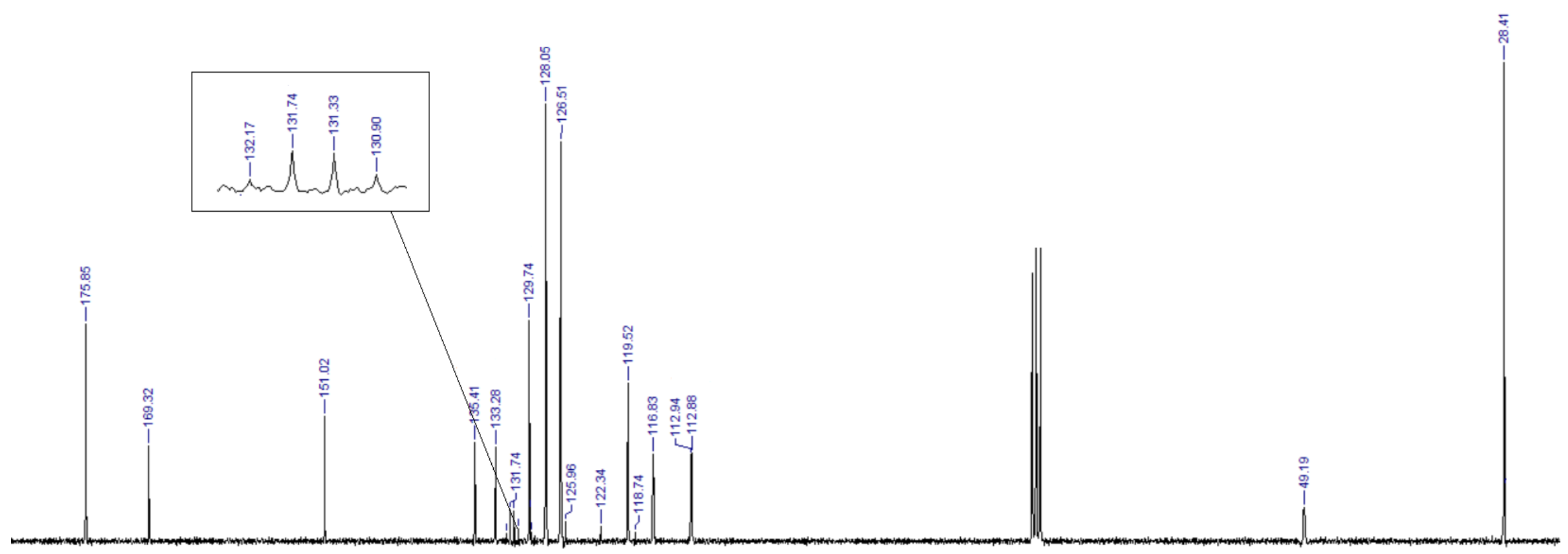

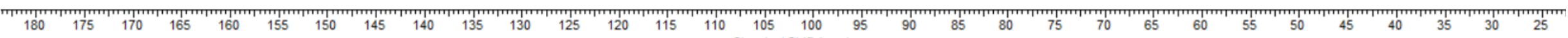


1-(4-(4-(3-(Trifluoromethyl)phenyl)piperazine-1-carbonyl)phenyl)pyrrolidine-2,5-dione (53) - ${ }^{19}$ F NMR
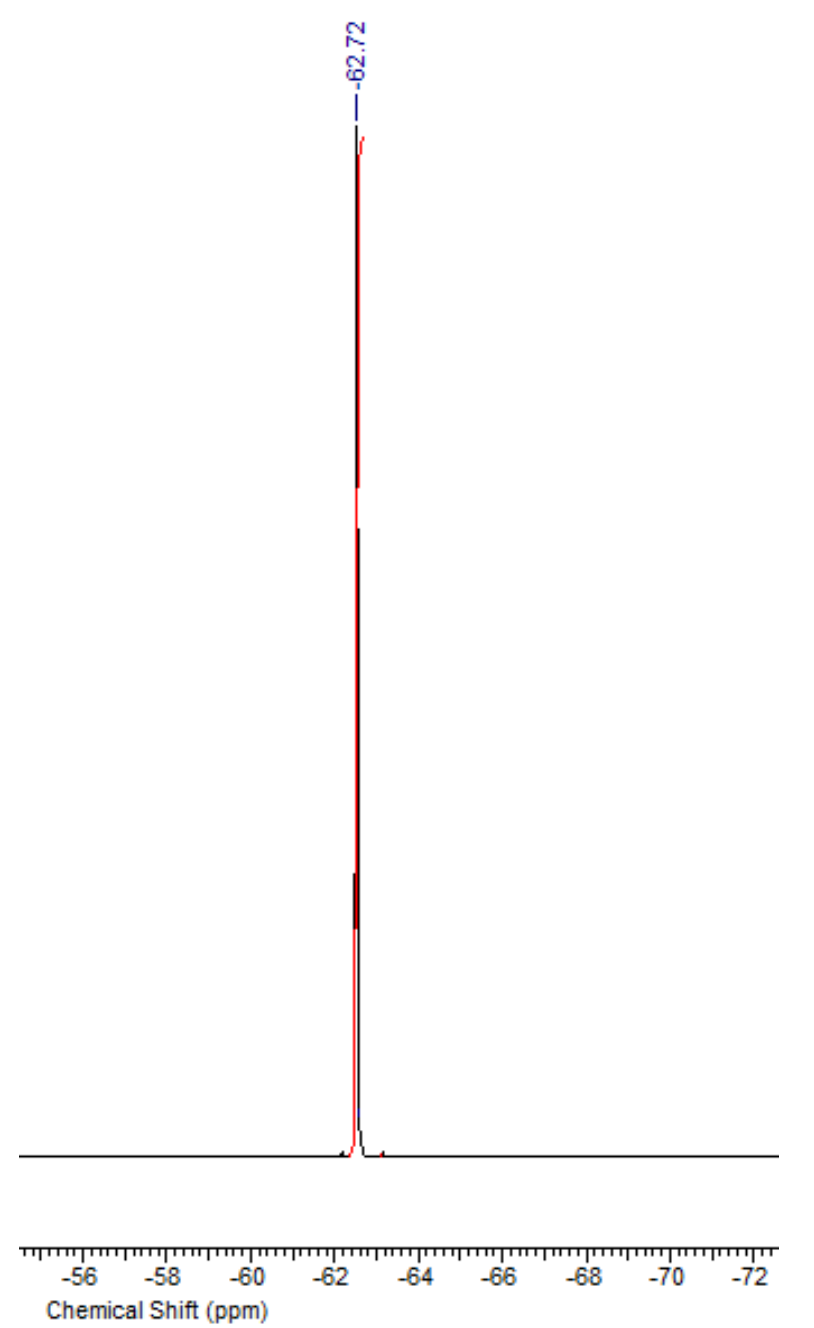